University of Louisville

ThinkIR: The University of Louisville's Institutional Repository

Electronic Theses and Dissertations

$12-2017$

\title{
PCB-associated steatohepatitis and the role of nuclear receptors.
}

Heather Brooke Clair

University of Louisville

Follow this and additional works at: https://ir.library.louisville.edu/etd

Part of the Nutritional and Metabolic Diseases Commons

\section{Recommended Citation}

Clair, Heather Brooke, "PCB-associated steatohepatitis and the role of nuclear receptors." (2017). Electronic Theses and Dissertations. Paper 2867.

https://doi.org/10.18297/etd/2867

This Doctoral Dissertation is brought to you for free and open access by ThinkIR: The University of Louisville's Institutional Repository. It has been accepted for inclusion in Electronic Theses and Dissertations by an authorized administrator of ThinkIR: The University of Louisville's Institutional Repository. This title appears here courtesy of the author, who has retained all other copyrights. For more information, please contact thinkir@louisville.edu. 
PCB-ASSOCIATED STEATOHEPATITIS AND THE ROLE OF NUCLEAR RECEPTORS

By

\author{
Heather Brooke Clair \\ B.S. University of Georgia, 1999 \\ M.S. University of Georgia, 2001 \\ M.S. University of Louisville, 2015 \\ A Dissertation \\ Submitted to the Faculty of the \\ School of Medicine of the University of Louisville \\ in Partial Fulfillment of the Requirements \\ for the Degree of \\ Doctor of Philosophy \\ In Biochemistry and Molecular Genetics \\ Department of Biochemistry and Molecular Genetics \\ University of Louisville \\ Louisville, KY
}

December, 2017 
Copyright 2017 by Heather Brooke Clair

All rights reserved 

PCB-ASSOCIATED STEATOHEPATITIS AND THE ROLE OF NUCLEAR RECEPTORS

By

Heather Brooke Clair

B.S. University of Georgia, 1999

M.S. University of Georgia, 2001

M.S. University of Louisville, 2015

A Dissertation Approved on

October 20, 2017

by the following Dissertation Committee:

\author{
Matthew C. Cave, M.D. \\ Russell A. Prough, Ph.D. \\ Barbara J. Clark, Ph.D. \\ Aruni Bhatnagar, Ph.D., FAHA \\ Juliane I. Arteel (nee Beier), Ph.D.
}




\section{DEDICATION}

For my family: you were my purpose and strength.

For my friends: you were my support and my shelter.

For my teachers: you stood as my examples.

For Michael, who is all three. 


\section{ACKNOWLEDGEMENTS}

I would like to thank the investigators and participants involved in the Anniston Community Health Survey for their tireless commitment to the health of those within their own community and beyond. I would also like to thank my department, committee members, and lab personnel for their help in the conception and execution of these projects, as well as the preparation of this publication. 


\section{ABSTRACT \\ PCB-ASSOCIATED STEATOHEPATITIS AND THE ROLE OF NUCLEAR RECEPTORS Heather B. Clair}

May 17, 2017

Metabolic diseases, including fatty liver disease, hyperglycemia, and obesity, result when body systems responsible for managing allostasis (dynamic homeostasis across systems) are pressured beyond their collective compensatory reserve. Nutritional excess contributes to this state, the capacity of which is limited by genetic variation, and failure of one system will gradually lead to pathological overload in the others. Agents which act directly on the communication machinery linking these connected systems can also change the point at which allostatic load becomes allostatic overload. Environmental exposure to polychlorinated biphenyls (PCBs), a class of persistent organic pollutant, is associated with a specific form of toxicant-associated steatohepatitis, fatty liver disease with inflammatory infiltration. PCBs are known to be ligands for the xenobiotic receptors, which, when activated, modulate the transcription of both xenobiotic and intermediary metabolic targets. We investigated the prevalence and characteristics of liver disease in a human population with high environmental PCB exposure, transcriptional changes in the liver in a mouse model of $\mathrm{PCB} /$ high-fat diet coexposure, and transcriptional changes attributable to xenobiotic receptors in a primary hepatocyte model. 
TABLE OF CONTENTS

PAGE

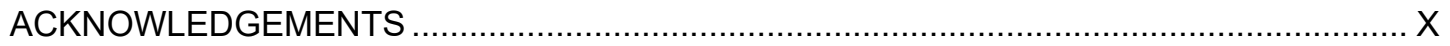

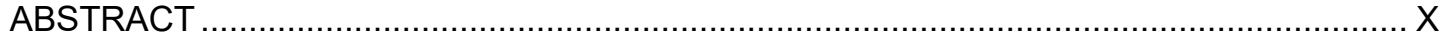

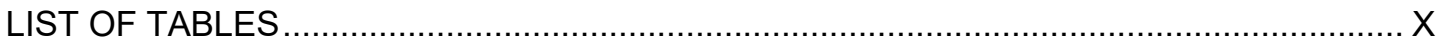

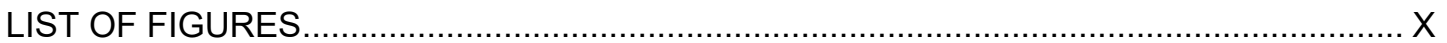

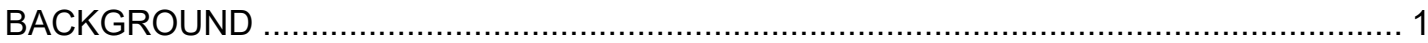

CHAPTER ONE: PCBs are associated with toxicant-associated steatohepatitis, inflammation, and metabolic dysregulation in an exposed human population ......................... 24

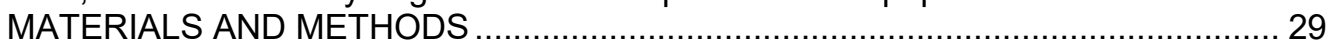

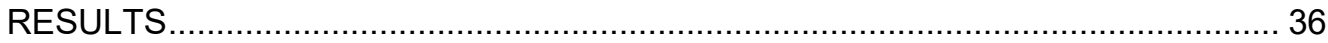

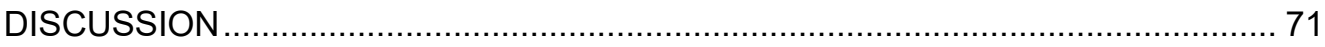

CHAPTER TWO: PCBs induce differential transcription in liver tissue from a mouse model of

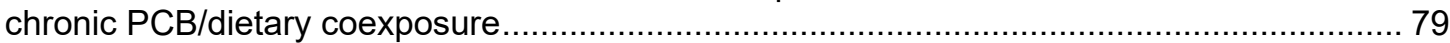

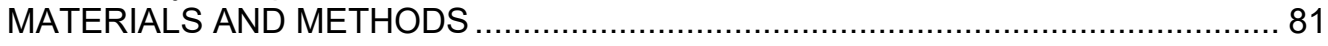

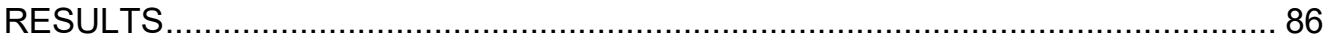

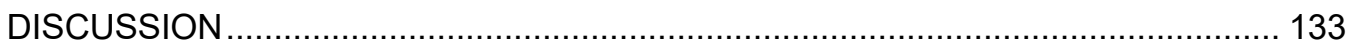

CHAPTER THREE: PCB exposure induces a differential transcriptome which partially overlaps with that of prototypical xenobiotic receptor ligands in a mouse primary

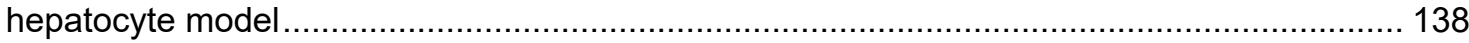

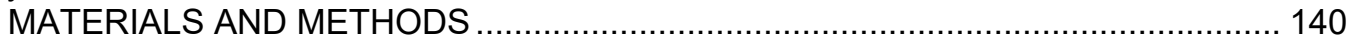

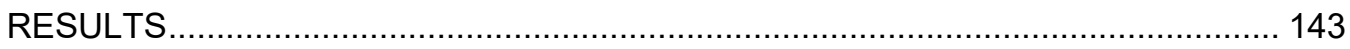

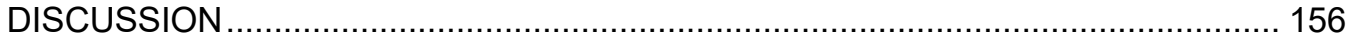

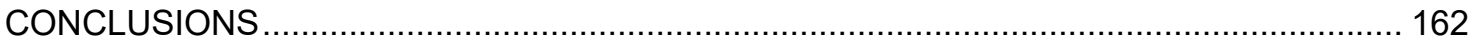

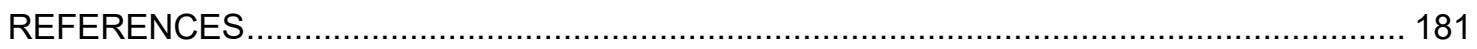

APPENDIX

Appendix Table 1. Fold change and summary information for 123 targets differentially transcribed with Aroclor 1260 exposure compared to prototypical ligand exposure .......... 213

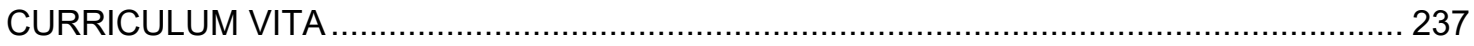




\section{LIST OF TABLES}

TABLE

PAGE

1. Composition of commercial Aroclor mixtures by PCB homolog group .............................. 5

2. Structural/functional groupings of PCB congeners measured in ACHS-II ......................... 11

3. 2003-2004 NHANES data - AOR for unexplained ALT elevation by exposure quartile.

4. Criteria for the diagnosis of Metabolic Syndrome (MS/CVD and IR/T2DM)

5. PCB congeners associated with AOR for unexplained ALT elevations in NHANES 2003-2004.

6. Demographics in ACHS by liver disease status - continuous variables 37

7. Demographics in ACHS by liver disease status - biometric categorical variables 38

8. Demographics in ACHS by liver disease status - lifestyle categorical variables. 39

9. Demographics in ACHS-II by liver disease status - continuous variables. 40

10. Demographics in ACHS-II by liver disease status - biometric categorical variables 41

11: Demographics in ACHS-II by liver disease status - health and lifestyle categorical variables.

12: Demographic characteristics and liver disease status of ACHS microRNA subcohort ..... 43

13: Univariate associations between demographic/exposure variables and the serum cytokeratin 18 M65 and M30 biomarkers used to categorize liver disease status 44

14: Unadjusted ACHS biomarker levels by liver disease status 46

15: Adjusted beta coefficients of associations of $\Sigma$ PCBs (wet weight) and liver status with inflammatory biomarkers

16: Adjusted beta coefficients of associations of $\mathrm{PPCBs}$ (wet weight) and liver status with metabolic biomarkers

17: ACHS validation - miRNA data by CK18 liver disease group 51

18: Adjusted beta coefficients of CK18 and miRNA levels 52

19: ACHS-II validation - LFTs by CK18 liver disease group 53 
20: Cutoff values for upper limit of normal for the clinical liver injury biomarker alanine aminotransferase (ALT)

21: Effects of PCBs and BMI on liver disease status by multinomial logistic regression 56

22: PCB groupings and CK18 liver disease status in ACHS and ACHS-II..... 57

23: Significant associations of $\Sigma P C B s$ and miRNA species 58

24: ACHS adjusted beta coefficients of significant associations of liver disease status and CK18 biomarkers by individual PCB congeners 60

25: TEQ for dioxin-like PCBs and other dioxin-like species and CK18-indicated liver disease status in ACHS-II

26: Adjusted beta coefficients of significant associations of various TEQ summations and CK18 biomarkers in ACHS-II ....

27: Unadjusted ACHS-II biomarker levels by sum of all PCBs ( $\Sigma \mathrm{PCBs}$ ) sum of 35 ortho-substituted congeners ( $\mathrm{PPCB}-\mathrm{O})$ and all non-dioxin-like congeners ( $\Sigma \mathrm{NDL})$

28: Unadjusted ACHS-II biomarker levels by groups of ortho-substituted PCBs 66

29: Unadjusted ACHS-II biomarker levels by groups of non-ortho-substituted and dioxin-like PCBs.

30: ACHS adjusted beta coefficients of associations of $\Sigma$ PCBs (wet weight) with biomarkers

31: Adjusted beta coefficents of significant associations of PCB congeners with biomarkers of glucose metabolism

32: in vivo experiment dataset properties. 85

33: Enrichments for genes differentially transcribed in comparison $\mathrm{CVvsC20}$ 87

34: Enrichments for genes differentially transcribed in comparison CVvsC200 88

35: Enrichments for genes differentially transcribed in comparison $\mathrm{HVvsH} 20$ 89

36: Enrichments for genes differentially transcribed in comparison $\mathrm{HVvsH} 200$... 90

37: Enrichments for genes differentially transcribed in comparison CVvsHV 91

38: Enrichments for genes differentially transcribed in comparison $\mathrm{C} 2 \mathrm{OvsH} 20$ 92

39: Enrichments for genes differentially transcribed in comparison $\mathrm{C} 200 \mathrm{vs} \mathrm{H} 200$ 93

40: Common targets between moderate/high PCB exposure levels within the control diet groups. 97 
41: Common targets between moderate/high PCB exposure levels within the high-fat diet groups

42: Common targets between all PCB exposure levels within diet comparisons 101

43: Enrichments for liver and metabolic disease endpoints in comparison CVvsC20 ............ 102

44: Enrichments for liver and metabolic disease endpoints in comparison CVvsC200 .......... 103

45: Enrichments for liver and metabolic disease endpoints in comparison $\mathrm{HVvsH} 20 \ldots \ldots \ldots \ldots . . . .104$

46: Enrichments for liver and metabolic disease endpoints in comparison HVvsH200.......... 105

47: Targets associated with liver disease enrichments and change vs. vehicle control by comparison.

48: Targets associated with metabolic disease enrichments (overnutrition/obesity) and their change vs. vehicle control in each comparison ....

49: Targets associated with metabolic disease enrichments (Type 2 Diabetes Mellitus)

and their change vs. vehicle control in each comparison

50: Targets associated with metabolic disease enrichments (Metabolic Syndrome)

and their change vs. vehicle control in each comparison

51: Targets associated with metabolic disease enrichments (insulin resistance)

and their change vs. vehicle control in each comparison

52: Top ten transcription factor enrichments for condition CVvsC20

53: Top ten transcription factor enrichments for condition CVvsC200

54: Top ten transcription factor enrichments for condition $\mathrm{HVvsH} 20$

55: Top ten transcription factor enrichments for condition $\mathrm{HVvsH200}$

56: Percent activation for engaged transcription factors identified in all conditions 125

57: Percent activation for engaged transcription factors identified in 1-3 conditions 126

58: Treatment summary.

59: Targets differentially regulated with Aroclor 1260 exposure.

60: Concordance in direction of differential transcription between PCB-DTGs

and prototypical ligand DTGs.

61: Fold induction of protypical targets for AhR, PXR, and CAR with prototypical

ligand exposure.

62: Enriched pathways for PCB-DTG set 
63: Transcription factors with targets in the PCB-dependent differentiallytranscribed gene set.

64: Differentially transcribed circadian-rhythm related targets from in vivo and in vitro experiments 


\section{LIST OF FIGURES}

FIGURE

PAGE

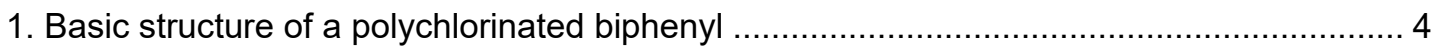

2. Structural characteristics of PCBs compared to TCDD and other dioxin-like chemicals..... 9

3. Equations for calculated parameters in human epidemiological studies .......................... 14

4: Distribution of ortho/non-ortho structures within homolog groups ................................. 17

5: Origin and detection of cytokeratin 18 in serum samples ................................................. 26

6: $\Sigma$ PCBs were inversely associated with metabolic biomarkers in ACHS............................ 64

7. Distribution of ALT levels in ACHS by liver disease category ....................................... 73

8. PCB exposure and high-fat diet induce phenotypic changes in C57/BL6 mice ................... 82

9: Moderate and high PCB exposures produce overlapping DTG sets (vs. vehicle) ............. 95

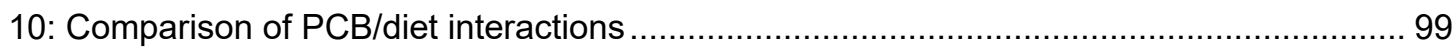

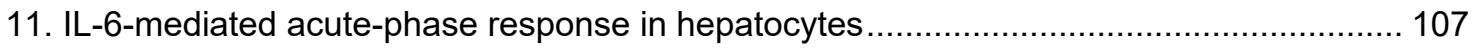

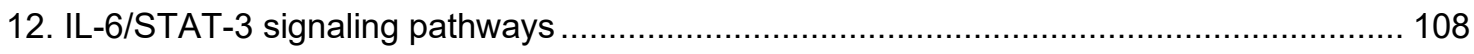

13. Transcription factors regulated by $P X R$ or $P X R / R X R$ via protein-protein

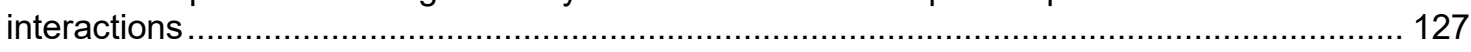

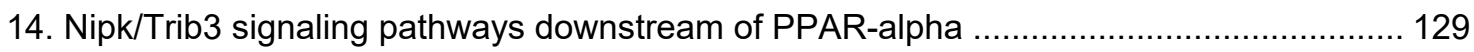

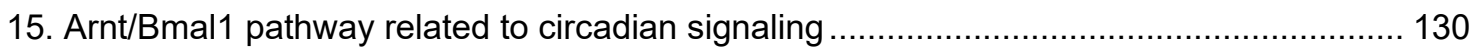

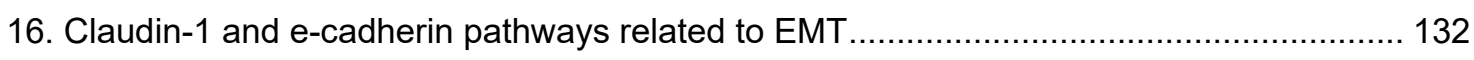

17. Summary of transcriptional differences between groups ......................................... 134

18. Histograms of differential transcriptomes produced by PCB/prototypical ligand exposure.

19. Venn diagram showing the overlapping differential gene sets between PCB and prototypical ligand exposures

20. A portion of the PCB-DTG overlapped with DTGs for AhR, PXR, CAR and

LXR prototypical ligands. 148 
21. Pathways related to EMT were enriched in genes in the PCB-DTG set ......................... 154

22. Exposure to PCB with or without HFD coexposure alters transcription of

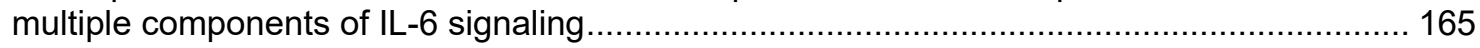

23. Ephrin ligand A1 (Efna1) was differentially regulated in multiple comparisons ................ 172

24. Ephrin ligand A5 (Efna5) was differentially regulated in multiple comparisons ................ 173

25. Ephrin receptor B2 (EphB2) was differentially regulated in multiple comparisons ............ 174

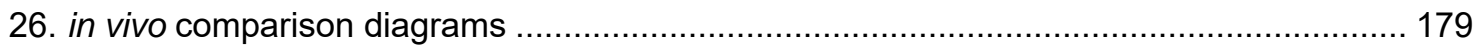




\section{BACKGROUND}

Toxicant-associated steatohepatitis (TASH) is a term applied to a unique subset of nonalcoholic fatty liver disease (NAFLD) related to chemical exposures. Though the liver is known to be a primary target in many industrial chemical exposures, environmental chemical exposures represent a much more widespread and insidious threat to liver health. Persistent organic pollutants (POPs) are lipophilic, and while some compounds are metabolized, relatively unmetabolizable chemicals distribute to fat depots of exposed biota. Consumption of these POPs-bearing biota represents the major route of environmental toxicant exposure for humans and other apex consumers. Consequently, even though the same classes of chemicals may be involved in industrial and environmental exposures, an environmental toxicant exposome is acquired slowly throughout the life cycle and is enriched in less metabolizable chemicals, while

an industrially-acquired exposome more likely involves higher levels of more readily metabolized chemicals acquired acutely by adults. This is an important consideration, because the same endpoints are used to evaluate toxicity in either case, but the differences in acquisition timeframe and composition may render the effects of chronic exposures subclinical and change toxicity mechanisms. These differences can affect the biomarkers used for screening assays, hindering diagnosis and treatment of TAFLD and TASH at earlier, more reversible stages and thereby masking the extent of liver injury.

The class of POPs collectively referred to as polychlorinated biphenyls (PCBs) typify the most challenging aspects of research in environmentally-acquired toxicant exposures. 1) Because of their chemical stability, PCBs continue to persist in the environment and in the tissues of humans decades after discontinuation of production. 2) PCBs are a class of chemicals invariably encountered as mixtures both in industrial and environmental exposures, and the 
structures present within those mixtures are diverse enough that they are likely to have multiple and potentially overlapping or competing mechanisms of toxicity. 3) Although compositions of these mixtures have been exhaustively documented in production, the environment, and in the tissues of biota including humans, for historical and political reasons, both the mixtures themselves and their effects are still often summarized by a single component which assumes one mechanism: in this case the subset of congeners bearing structural similarity to dioxins and expected to act as arylhydrocarbon receptor (AhR) agonists. 4) The direct effects of chronic, lowlevel PCB exposures differ from the more thoroughly evaluated effects of high-level, industrial exposures - likely because different distributions of congeners drive different engagement of xenobiotic receptors. 5) The ultimate effects of chronic PCB exposure-related TASH have great potential to be modulated or exacerbated by long-term nutritional coexposures, and race/ethnicity and gender differences, and these differences are understudied. 6) PCBs, like other toxicants associated with TASH, appear to cause a form of liver disease which is mechanistically and diagnostically distinct from other forms of fatty liver disease, making its detection by typical laboratory screening techniques difficult. These points may explain some characteristics which frustrate the recognition of environmentally-acquired PCB impact on human health and hinder its resolution.

PCBs remain a focus of environmental and human health research, occupying (in various groupings) 3 out of the top 20 positions in the 2015 Substance Priority List published by the Agency for Toxic Substances Disease Registry (ATSDR) ${ }^{1}$. We hypothesized that, in humans, chronic environmental PCB exposure would be associated with increased liver injury biomarkers indicating TASH. We furthermore hypothesized that PCB exposure in mice and primary mouse hepatocytes would induce differential transcription of targets related to liver disease and that in primary mouse hepatocytes, that differential transcription would overlap with the response to prototypical ligands for specific xenobiotic receptors. 


\section{Manufacture and chemical characteristics of PCBs}

Every human health effect of PCBs must be evaluated in light of one critical consideration: from the moment of manufacture to the moment of exposure, PCBs never exist as a single chemical, but as a fluctuating mixture of related chemicals each with unique biological and chemical properties. The basic structure of a PCB, shown in Figure 1, is that of a biphenyl ring with as few as 1 or as many as 10 chlorine substitutions in various positions about the molecule, leading to 209 potential congener configurations. PCBs were produced throughout the world via ferric chloride-catalyzed chlorination of biphenyl. Three cities in the U.S. were sites for commercial manufacture of PCBs: Anniston, AL from 1929-1930 (Swann Research, Inc.) and 1930-1971 (Monsanto, Inc.), Saguet, IL until 1977 (Monsanto, Inc.) and Houston, TX from 19721974 (Geneva Industries)². PCBs were produced mainly for use as dielectric fluids in electrical equipment, but were also used as lubricants, plasticizers, sealants and carriers in many products. The desired material properties of chemical stability and low flammability offered by PCB mixtures are related to the component structures and correspond roughly to the degree of chlorination. PCBs produced by Monsanto, Inc. were marketed as mixtures called Aroclors, with a number corresponding to the nominal average percent chlorine (e.g. Aroclor 1260 is approximately $60 \%$ chlorine by weight) ${ }^{3}$.

This production and marketing methodology gives rise to one scheme for grouping PCBs: PCB homologs are defined as sets of congeners with a common number of chlorine substitutions. In addition to providing a way to estimate levels of total production, use and disposal of PCBs and to equate these amounts with contamination levels, grouping by homologs allows the appreciation of certain general characteristics and trends. More heavily substituted homologs have higher average melting and boiling points, higher vapor pressure and are more miscible in lipids (quantified as higher octanol-water partition coefficients) ${ }^{3}$. Aroclor mixtures including Aroclor 1260, 1254 and 1248 were composed mainly of higher-molecular weight homologs (tetrachlorobiphenyl and above). Within each homolog group, PCB congeners with different substitution patterns are referred to as isomers. The composition of commercial Aroclor mixtures by PCB homolog group is shown in Table 1. 


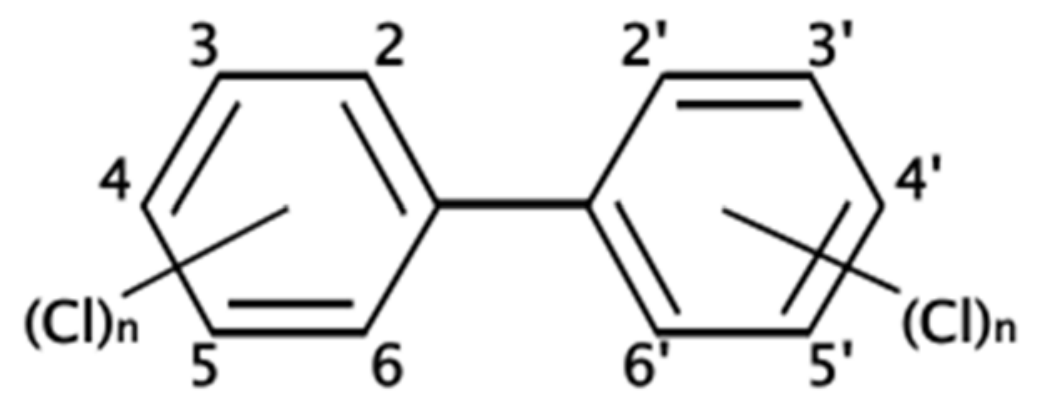

Figure 1. Basic structure of a polychlorinated biphenyl.

The basic structure of a PCB is a biphenyl ring with 1-10 chlorine substitutions at carbons 2 (2') and $6\left(6^{\prime}\right)$. Substitutions in the ortho positions favor rotation of the rings relative to one another, with the functional consequence of reduced coplanarity with each additional ortho substitution. PCB congeners with less (tri, di, mono-ortho) or no ortho substitutions (non-ortho) assume an increasingly more coplanar structure. 


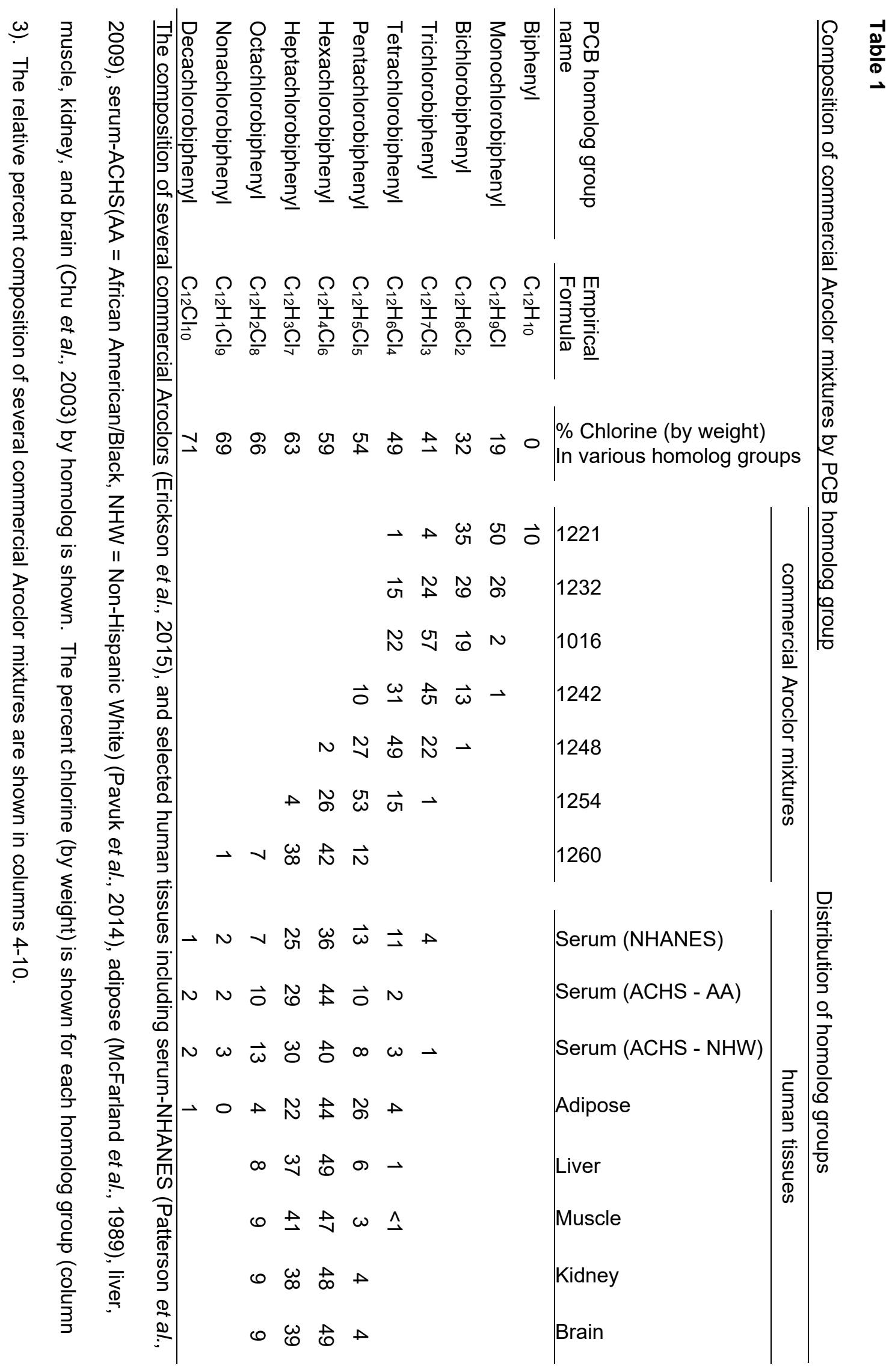


Unsurprisingly, the environmental persistence of individual congeners within the mixture is also related to structure. Weathering is a term applied to PCB mixtures undergoing profile changes due to physical or chemical processes such as water washing, photodegradation, volatilization, and evaporation. It can also refer to aerobic degradation due to microbial action. Weathering disproportionately affects lighter molecular weight congeners, particularly mono-, di-, and tri- substituted congeners, resulting in mixtures with proportionately more tetra- and pentasubstituted congeners ${ }^{4}$. Congeners bioaccumulating within the fatty tissue of exposed biota also tend to be those with more chlorine substitutions, and are dependent on two properties related to structure and influenced by degree of chlorination: resistance to non-metabolic clearance (increased lipophilicity) and structural inaccessibility to metabolic machinery (substitution pattern $)^{5}$.

In summary, PCBs were manufactured to meet a demand for industrial fluids that were highly stable, thermoresistant, and miscible in lipids, the same characteristics underlying the persistence of PCBs in the environment. It is important to note, however, that PCB congeners are not equally affected by processes of physical and chemical degradation, leading to changes in the profile of PCB mixtures from manufacture to environmental substrates to eventual bioaccumulation in humans. This is particularly critical to the design of models which reflect the current PCB exposure conditions: uptake of weathered and pre-metabolized PCBs through ingestion of contaminated biota.

Bioactivity of PCBs in mixtures with widespread relevance to human health

Over 80 years have elapsed since the initial reports of human toxicity from PCB exposure $^{3,6}$, and in that time, substantial research efforts have been put toward understanding the chemical composition and health effects of manufactured PCB mixtures and occupational exposures $^{7-12}$. While this work has been critical to characterizing human disease resulting from industrial exposures, it has become somewhat obsolete for several reasons. First, except from a standpoint of longitudinal health and mortality assessment, research modeled on occupational PCB exposures is less relevant today because PCB production has been banned for 40 years, 
therefore, new occupational exposures no longer occur in the process of PCB manufacture. Second, the most common route of exposure in humans is low-level, environmental acquisition through ingestion of contaminated foodstuffs, and as congeners bioaccumulate at different rates, PCBs which make up the current exposome are more likely to be higher-substituted, highlybioaccumulated and less metabolizable, generalizations which are reflected in assessments of serum PCB levels in exposed populations. Third, emerging data on the impact of nutritional coexposures suggests that the current obesity epidemic may significantly modulate the effects of PCBs on human health. Thus, different PCBs may be exerting their effects in a different internal environment currently than was explored in the original toxicity studies.

Early evaluation of the toxicity of PCBs focused on high-level exposures and acute toxicity which modeled the human health effects seen in occupational exposures ${ }^{7-9}$, accidental contamination from PCB-containing electrical equipment ${ }^{12,13}$, and poisoning incidents such as the Yusho and Yucheng 9 , 14, 15 events in which thousands of people in Japan and Taiwan (respectively) were exposed to PCBs, dioxins and dibenzofurans through ingestion of rice oil contaminated with a commercial PCB mixture. These incidents differ from the PCB exposure experienced by most U.S. citizens in both scale and composition. Health consequences such as chloracne (a noninflammatory skin condition characterized by cyst and closed comedone development with sebaceous gland atrophy), immunosuppression, reproductive and developmental effects, acute liver damage, and cancer were observed in some individuals within these cohorts. Importantly, elevated liver enzyme activity and other biomarkers of these effects were typically observed at very high exposures. This observation as well as a superficial resemblance of this pattern of human health effects to the constellation of pathologies induced by dioxins in laboratory animals ${ }^{16}$ led researchers to focus on a small subset of congeners within the exposure mixture with a specific structural characteristic: absence of chlorine substitutions in the four available ortho positions. This characteristic is related to but independent of the overall degree of chlorination in the molecule. Chlorine substitutions at these ortho positions affect the orientation of the rings relative to one another such that congeners with no substitutions in these 
positions are relatively coplanar, while increasing numbers of chlorine substitutions in these positions increases the likelihood of a molecule with rings that are non-coplanar (perpendicular).

Coplanar PCBs share structural similarity to the molecule 2,3,7,8-tetrachlorodibenzo-pdioxin (TCDD), which is an extensively studied, potent agonist for the AhR. Structural characteristics of PCBs compared to TCDD and other dioxin-like chemicals are shown in Figure 2. Coplanar PCBs, which can induce transactivation of targets under the control of a dioxin response element (DRE), are considered dioxin-like (DL) PCBs, while increasing ortho substitutions render a congener increasingly non-dioxin-like (NDL). The number of relatively potent (non-ortho) dioxin-like congeners is constrained by the requirement for unsubstituted ortho carbons (2, 2', 4, and 4') and becomes increasingly unlikely as the number of substitutions increases, such that only 1 non-ortho congener (PCB 169) exists among hexachlorobiphenyls and none exist among the higher molecular weight congeners.

Most research has focused on these dioxin-like congeners, which are present in only trace amounts within most commercial PCB mixtures and found at extraordinarily low levels in the tissues of exposed biota including humans. In rodent models, exposure to TCDD has been linked to many of the disease endpoints listed above, including immunotoxicity, reproductive effects, and cancer $^{16}$, although the connection between TCDD and other dioxins and these effects in humans is less clear ${ }^{17}$. Even chloracne, which is the only consistently-reported human health marker of dioxin intoxication ${ }^{18}$, is not a clear outcome of $\mathrm{AhR}$ induction and does not follow AhR induction by all ligands. Nevertheless, coplanar PCBs are also ligands for AhR, although they are much less effective than prototypical ligand TCDD at inducing transcription of Cyp1a1, the prototypical target of $\mathrm{AhR}$, in vivo ${ }^{16}$.

NDL congeners are characterized by chlorine substitutions in the ortho positions, which induce non-coplanarity. These congeners tend to be more resistant to degradation and bioaccumulate to much higher levels. Historically, these congeners were dismissed as less toxic to humans and of a lower priority in remediation and human health research ${ }^{16}$, however more recently, the recognition that these congeners are ligand activators of other xenobiotic receptors has led to a resurgence of interest. NDL congeners are direct ligands for the pregnane and 


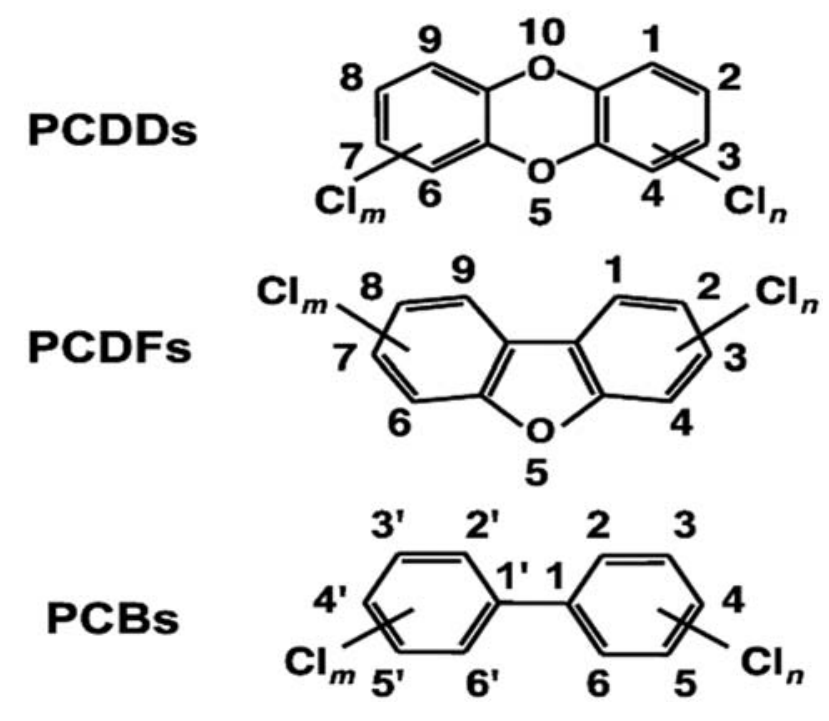

Figure 2. Structural characteristics of PCBs compared to TCDD and other dioxin-like chemicals.

(Adapted from Int. J. Mol. Sci. 2014, 15(8), 14044-14057.) 
xenobiotic receptor (PXR) and the human constitutive androstane receptor isoforms 2 and 3 (hCAR2/3) but not human CAR isoform 1 (hCAR1), which is homologous to murine CAR $(\mathrm{mCAR})^{19}$. However, our laboratory recently reported that the both NDL and DL PCB congeners, as well as the mixture Aroclor 1260, inhibit activation of the human and murine epidermal growth factor receptor (EGFR), which provides an indirect mechanism for mCAR activation $20,21$.

Importantly, though AhR, CAR, and PXR are considered xenobiotic receptors and most research has focused on describing their role in the drug- or toxicant-dependent induction of xenobiotic metabolic machinery, they are also involved in transcriptional control of intermediary metabolism and inflammatory response. These functions may ultimately explain both the link between PCB exposure and metabolic disruption and some complexities of diet/PCB coexposures in metabolic disease. Our laboratory reported that, in mice, exposure to Aroclor 1260 alone was insufficient to induce a phenotype of steatohepatitis, and that a coexposure to high fat diet was required to observe the phenotypes of fatty liver and diabetes ${ }^{22,23}$.

Beyond the DL/NDL stratification, other groupings of PCB congeners reflect the ability of individual congeners to alter the function of receptors such as the ryanodine receptor ${ }^{24-26}$, estrogen receptor 27,28 , and androgen receptor ${ }^{29,} 30$, shown in Table 2.

In summary, humans encounter PCBs not as individual chemicals, but as complex mixtures of congeners, each of which has unique structural characteristics that influence stability and bioaccumulation patterns as well as the mechanisms of biological effect. The composition of PCB mixtures represented in historic occupational and accidental PCB intoxication events differs significantly from the mixture accumulated slowly through chronic environmental exposure, and this is likely to alter both the mechanism of effect and the phenotype. Therefore, experimental designs which involve single congeners and risk-assessment algorithms which assume only one mechanism are unlikely to realistically model the effect of environmental exposure.

Historic context for reliance on AhR induction estimates to define PCB toxicity

As stated above, although PCBs are encountered as complex mixtures with complex effects, historically, the toxicity of PCBs was attributed entirely to AhR-mediated effects of dioxin- 
Table 2

Structural/functional groupings of PCB congeners measured in ACHS-II

\section{Structural groupings}

\begin{tabular}{|c|c|}
\hline All ( $\Sigma$ PCB) & $\begin{array}{l}28,44,49,52,66,74,87,99,101,105,110, \\
118,128,138-158,146,149,151,153,156, \\
157,167,170,172,177,178,180,183,187, \\
189,194,195,196-203,199,206,209\end{array}$ \\
\hline Ortho-Substituted ( $\Sigma$ PCB-O) & $\begin{array}{l}28,44,49,52,66,74,87,99,101,105,110, \\
118,128,138,146,149,151,153,156,157 \\
167,170,172,177,178,180,183,187,189, \\
194,195,196,199,206,209\end{array}$ \\
\hline Tri- and Tetra-ortho ( $\Sigma$ PCB-TO) & $\begin{array}{l}\text { 149, 151, 177, 178, 183, 187, 195, 196-203, } \\
199,206,209\end{array}$ \\
\hline Di-Ortho ( $\Sigma$ PCB-DO) & $\begin{array}{l}44,49,52,87,99,101,110,128,138-158,146, \\
153,170,172,180,194\end{array}$ \\
\hline Mono-Ortho ( $\Sigma$ PCB-MO) & $28,66,74,105,118,156,157,167,189$ \\
\hline 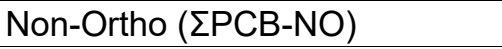 & $81,126,169$ \\
\hline
\end{tabular}

Functional groupings

\begin{tabular}{ll}
\hline Dioxin-Like $(\Sigma D L)$ & $81,105,118,156,157,167,189,126,169$ \\
\hline \multirow{2}{*}{ Non-Dioxin-Like $(\Sigma N D L)$} & $28,44,49,52,66,74,87,99,101,110,128$, \\
& $138,146,149,151,153,170,172,177,178$, \\
& $180,183,187,194,195,196,199,206,209$
\end{tabular}

Estrogen receptor (estrogenic) $\quad 18,28,30,44,49,52,99,101,103,110,128$

Estrogen receptor (anti-estrogenic) $\quad 118,138,163,170,180,187,194,199,203$

Androgen receptor (anti-androgenic) $\quad 28,49,66,74,105,118$

Ryanodine receptor

$95,136,149,176,84,96,52,151,183,187$ $170,101,132,180,18$

PCBs are grouped by structural and structural/functional characteristics to assess relationships between disease or biomarker parameters and the total serum levels of structures with common characteristics (structural) or mechanisms (structural/functional). For dioxin-like/non-dioxin-like congeners, the designation is based on the presence of ortho-substitutions ( 0 or 1 for dioxinlike, 2-4 for non-dioxin-like). 
like congeners. Both political and practical considerations motivated this focus. From a practical standpoint, acute occupational exposures and high-level accidental poisoning with relatively undegraded PCB mixtures were readily observable, and therefore exposure to mixtures containing lower molecular weight and dioxin-like congeners was the primary PCB-related human health concern at the time. Aggressive exploration of environmental PCB contamination began in the 1970's, following adoption of gas chromatography techniques which allowed identification of PCBs (as well as chlorinated pesticides) in the environment ${ }^{3}$. In the same general time, political pressure revolving around the use of Agent Orange in the Vietnam War steered a great deal of research toward the health effects of dioxins and dioxin-like chemicals. The result of this was the adoption of the TCDD-equivalent factor (TEF) protocol to determine the TCDD equivalency (TEQ), a value normalizing any dioxin-like compound to TCDD based on its ability to induce AhRmediated response in an in vivo or in vitro system ${ }^{31}$. Classes included in this protocol include PCDDs, PCDFs and PCBs, the structures of which are shown in Figure 2. While this system allows for the quick estimation of AhR-mediated toxicity in complex mixtures and clinical or environmental samples by summing concentrations of individual compounds multiplied by their respective TEQs, there are problems inherent in the concept.

First, the protocol presupposes that "Most, if not all, toxic and biological effects of [PCDDs, PCDFs and PCBs] are mediated through the aryl hydrocarbon receptor"31. This is certainly not true of mixtures such as PCBs in which all potential AhR ligands make up less than $1.5 \%$ of even unweathered commercial mixtures like $1260^{32}$. Far from being inert, the remaining $98.5 \%$ of this mixture contains congeners which are known to induce other receptors, including both classical xenobiotic nuclear receptors ${ }^{19,33-35}$ and cell surface receptors ${ }^{20}$. Second, classic assays based on induction or activity of xenobiotic metabolic targets of AhR presume that exposure-dependent changes in AhR-mediated induction in this subset of targets can be extrapolated to the entire complement of AhR targets. In fact, the transcriptional effects of AhR are selectively modified by the ligand, with more widely conserved effects across ligands on targets involved in xenobiotic metabolism or response to oxidative stress and more ligand-specific modulation of targets involved in lipid carbohydrate metabolism ${ }^{36}$. Finally, the most powerful 
application of the TEQ protocol is the ability to quickly summarize the AhR-mediated effects of various chemicals quantified by gas chromatography coupled to high-resolution mass spectrometry (GC/HRMS) by normalizing the contribution of each compound to TCDD using the TEF and then summing the resulting TEQs. This allows a total TEQ to be derived from multiple dioxin-like classes of chemical, including PCDDs, PCDFs and PCBs (Figure 3). Perhaps most pertinent to this particular study, although data from multiple human exposures to these compounds, including Yusho and Yucheng poisoning events ${ }^{14}$ and exposed individuals residing near the PCB manufacturing facility in Anniston, AL suggest that PCDDs and PCDFs contribute more to the overall TEQ. However, our laboratory's evaluation of the 2003-2004 NHANES data showed that, among these classes, after adjustment for multiple comparisons, only PCBs were positively associated with liver injury ${ }^{37}$ (shown in Table 3).

In summary, a need to prioritize exposures for reasons of risk assessment and remediation led to methods, like the TEQ protocol, which weight the importance of one mechanism over others, and the selection of dioxins/AhR as the flagship toxicant was driven by both practical and political considerations. While this method may accurately represent the likelihood that PCBs will have effects mediated by AhR, neither AhR activation nor modulation of any other single mechanism can sufficiently describe the toxic/biological effects of complex environmental exposures. This is particularly true in the case of PCBs, where both the overall exposure level and the distribution of congeners comprising the mixture are known to differ considerably between acute/occupational and chronic/environmental exposures.

\section{Environmental PCB exposures vs. industrial PCB exposures}

High-level exposures to industrial PCB mixtures are known to cause liver injury as well as pathologies in other tissues in humans and other animals, as previously described. Although the effects of chronic, environmental PCB exposures on the liver are less well characterized, some conclusions can be drawn about the relationship between PCBs and liver injury based on the results of several studies: the NHANES study described above, a longitudinal examination of the 
Equation 1. PCDD TEQ

$$
P C D D T E Q=\sum_{n i}\left(P C D D_{i} \times T E F_{i}\right)_{n}
$$

Equation 2. PCDF TEQ

$$
P C D F T E Q=\sum_{n i}\left(P C D F_{i} \times T E F_{i}\right)_{n}
$$

Equation 3. PCB TEQ

$$
P C B T E Q=\sum_{n i}\left(P C B_{i} \times T E F_{i}\right)_{n}
$$

Equation 4. Total TEQ

$$
\begin{aligned}
& \text { Total TEQ }=\sum_{n i}\left(P C D D_{i} \times T E F_{i}\right)_{n}+\sum_{n i}\left(P C D F_{i} \times T E F_{i}\right)_{n} \\
& \quad+\sum_{n i}\left(P C B_{i} \times T E F_{i}\right)_{n}
\end{aligned}
$$

Equation 5: HOMA-IR

$$
H O M A-I R=\frac{(\text { Glucose } X \text { Insulin })}{22.5}
$$

Equation 6: HOMA- $\beta$

$$
\text { HOMA }-\beta=\frac{(20 X \text { Insulin })}{(\text { Glucose }-3.5)} \%
$$

Equation 7: Total Lipids

Total Lipids $=(2.27 X$ Total Cholesterol $)+$ Triglycerides $+62.3 \mathrm{mg} / \mathrm{dl}$

Figure 3. Equations for calculated parameters in human epidemiological studies.

These equations are used to summarize and interpret differential biomarkers in human serum.

Equations 1-4 are used to calculate the effects of various PCB congener groupings on the arylhydrocarbon receptor. Equations $5 \& 6$ are used the in homeostatic method of assessment (HOMA) model to discriminate between different etiologies in abnormal glycemic control.

Equation 7 is used to estimate the total lipid content of serum from measured parameters of total cholesterol and triglycerides. "Total lipids" is then used as a variable to correct serum content of hydrophobic contaminants such as PCBs. 
Table 3

2003-2004 NHANES data - AOR for unexplained ALT elevation by exposure quartile

\begin{tabular}{|c|c|c|c|c|c|}
\hline \multirow{2}{*}{$\begin{array}{l}\text { Pollutant } \\
\text { Subclass }\end{array}$} & \multicolumn{4}{|c|}{$\begin{array}{c}\text { Adjusted Odds Ratios for } \\
\text { Unexplained ALT Elevation } \\
\text { by Exposure Quartile }\end{array}$} & \multirow{2}{*}{$\begin{array}{c}\mathbf{p}_{\text {trend- }}^{+} \\
\text {adj }^{\dagger}\end{array}$} \\
\hline & $1^{\text {st }}$ & $2^{\text {nd }}$ & $3^{\text {rd }}$ & $4^{\text {th }}$ & \\
\hline Coplanar PCBs & Ref & 2.2 & 4.4 & 7.6 & $<0.001$ \\
\hline $\begin{array}{l}\text { Non-dioxin-like } \\
\text { PCBs }\end{array}$ & Ref & 0.8 & 2.4 & 4.5 & 0.001 \\
\hline Total PCBs & Ref & 0.8 & 2.2 & 4.3 & 0.010 \\
\hline
\end{tabular}

The relationships between biomarker-indicated liver disease and PCBs in the 2003-2004

NHANES population are shown. Increased total PCB exposure was associated with an increased adjusted odds ratio (AOR) for unexplained ALT elevation, attributable to nonalcoholic fatty liver disease. From Cave et al, 2010. Used with permission. 
historically exposed Yusho population ${ }^{12}$, PCB exposure in the Michigan fisheaters study ${ }^{38}$, and data from exposed wildlife ${ }^{16,39}$.

At higher molecular weights, the distribution of congeners heavily disfavors non-ortho, strong AhR ligands. Bioaccumulation patterns favor higher molecular weight congeners, meaning that, for humans acquiring PCBs through the ingestion of contaminated biota, the initial exposure is to a higher average molecular weight, more ortho mixture (shown in Table 1), and the steady state distribution of congeners further reinforces this pattern, shown in Figure 4 and Table 1.

Sex, genotype and diet interactions in PCB-exposed humans and animal models

From a standpoint of complexity, there is no more daunting area of human health research than metabolic disease. The metabolic machinery of humans is both versatile and robust. We can adapt to frequent physiological fluctuations in nutritional status, different food sources, and varying demands from multiple tissues to support daily function, development, and reproduction. In many cases, tissues can weather temporary metabolic disruption due to illness or intoxication and eventually regain homeostasis. This process is described by the concept of allostasis, or maintaining stability through change, an energy-dependent process involving concerted actions between multiple tissues ${ }^{40}$. On the other hand, in the multistep and multisystem process that converts nutrients into metabolites and energy, a mutation leading to loss of function in one critical enzyme can eventually lead to failure in connected systems. Complex disease etiologies occur where additive pressures across connected systems eventually overwhelm compensatory processes. Eventually, component systems fail as additive pressures overwhelm their capacity to adapt, meaning that pressure from different causes can converge on shared pathological outcomes. Because of the multitude of processes in play, disease progression may be slow and pleiotropic, and the direct effects of causal agents may be obscured as the allostatic load is shunted to other systems.

In mammals, the liver is the primary biochemical interface between the environment and the organism, transducing signals and substrates in multiple directions to sense and respond to constantly-changing conditions. In terms of allostatic processes, this places the liver as both a 


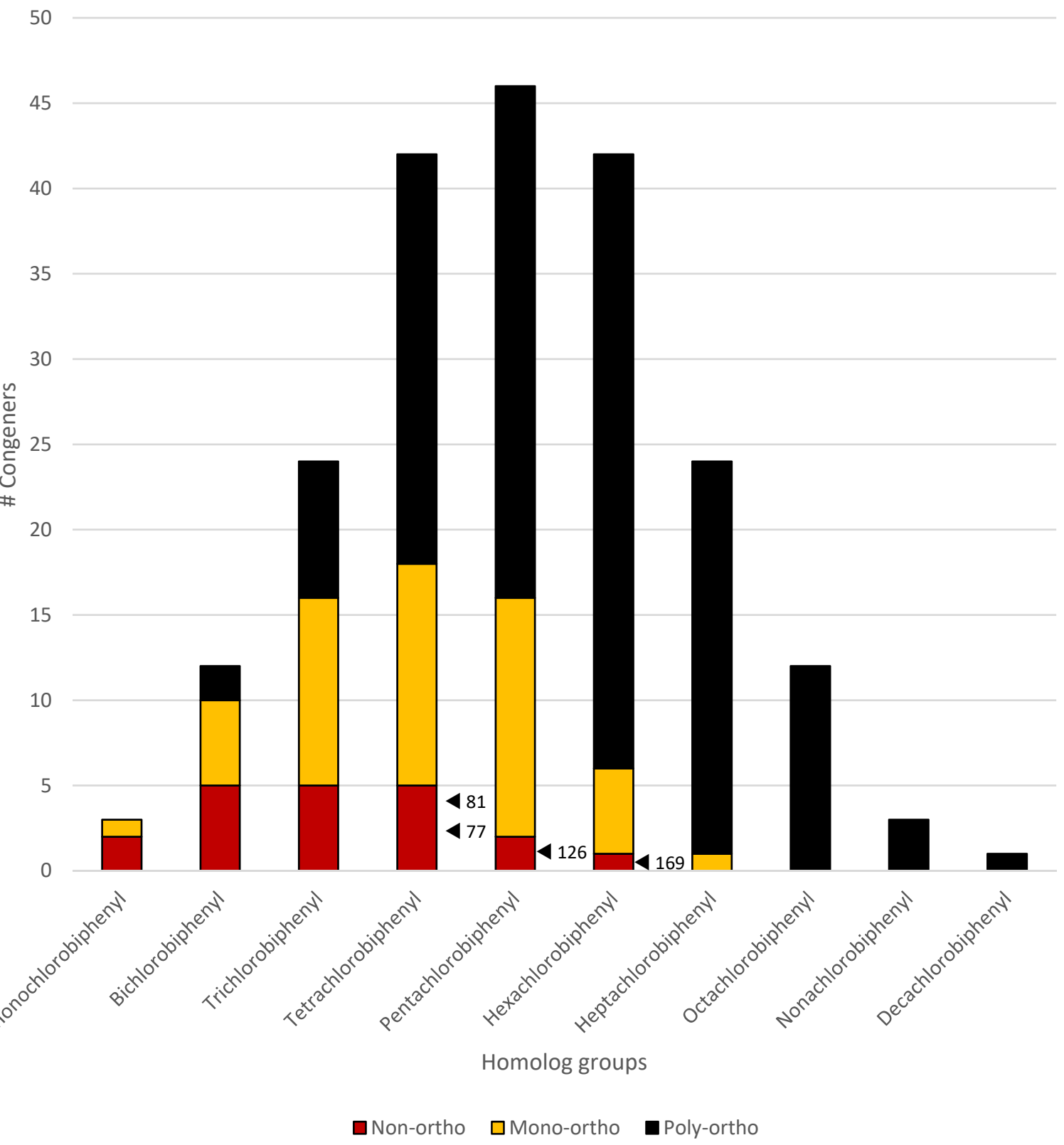

Figure 4. Distribution of ortho/non-ortho structures within homolog groups.

As molecular weight and number of chlorine substitutions increases, ortho substitutions become more likely. The four non-ortho substituted congeners which bioaccumulate to appreciable levels in humans are PCBs 77, 81, 126 and 169. 
hub (because of its position in lipid and carbohydrate trafficking) and as a temporary terminus (because of its capacity for lipid and carbohydrate storage). The adaptive capacity of the liver is extraordinary, but not limitless. Lipid and carbohydrate uptake which chronically exceeds hepatic metabolic/export capacity can lead to steatosis. This has been demonstrated experimentally, using diets enriched in various fats or sugars to induce steatosis, (as well as obesity, dyslipidemia, hyperglycemia and hyperinsulinemia) in laboratory animals ${ }^{41-44}$. The process is also indicated in humans by epidemiological studies which show a strong relationship between BMI or visceral obesity (proxy measurements for hypercaloric/high-fat/high-sugar diets) and metabolic dysregulation. In addition to the higher PCB exposures found in Anniston and other exposed human populations vs. the NHANES 2003-2004 population, the contributions of overweight/obesity to steatohepatitis and other metabolic disease cannot be ignored. These nutritional factors are additive stressors, increasing demand on tissues whose adaptive capacity is compromised by the effects of sensing or responding to xenobiotics. The boundaries of that capacity arise from genetic variation in the components of metabolic machinery and the combination of endobiotic and xenobiotic molecules interpreted as a need for functional change. Examples drawn from epidemiological studies include patatin-like phosphospholipase domaincontaining-protein 3 (PNPLA3), which has been shown to segregate with racial/ethnic variations in NAFLD prevalence ${ }^{45}$ and the prevalent Q84R (missense) mutation in the human tribbles pseudokinase 3 gene (TRIB3) which is associated with insulin resistance/T2DM ${ }^{46,47}$, cardiovascular risk ${ }^{46}$, and polycystic ovary syndrome in various human populations. Humans homozygous for dominant-negative PPARY (adipose-prevalent isoform) have reduced body fat and greatly increased insulin resistance ${ }^{48}$. Sex effects are also clear in epidemiological studies of PCB-associated disease. In exposed individuals from Anniston, AL, T2DM prevalence was increased only in females ${ }^{49}$, a finding which echoed reports from a 24-year follow-up of the historic Yucheng poisoning incident, which found a 2-fold increase in the prevalence of diabetes among female but not male subjects ${ }^{50}$. Males in the Yucheng cohort had a higher prevalence of mortality from liver cirrhosis and liver cancer ${ }^{51}$. 
In summary, additive pressures from chemical exposures and nutrition can combine with sex- and genotype- specific variation in adaptive capacity to change the prevalence or the manifestation of metabolic disease. Because of its central position in both allostatic maintenance and metabolic and detoxification processes, the liver might be expected to show early and extensive effects from the multiple "hits" of xenobiotic and dietary exposures.

\section{PCBs and TASH}

The extraordinary range of molecular structures which can be recognized and successfully handled by one or more hepatic metabolic pathways is rivaled only by another unique quality: the liver can really take a beating. The liver can successfully regenerate after surgical removal more than $70 \%$ of its total mass ${ }^{52,53}$, or the necrosis of $1 / 3$ of the hepatic lobule following exposure to toxicants such as acetaminophen or carbon tetrachloride ${ }^{54}$.

Nonalcoholic fatty liver disease (NAFLD) is a widespread metabolic disease of the liver, affecting an estimated $25 \%$ of the population worldwide ${ }^{55}$. NAFLD is defined histologically by steatosis (pathological accumulation of lipids) within $5 \%$ or more of hepatocytes in the absence of excess alcohol consumption. First considering the liver as an isolated system, four general mechanisms can initiate lipid accumulation: increased lipid transport into the hepatocyte, increased de novo synthesis of lipids, decreased $\beta$-oxidation, or decreased lipid export ${ }^{56}$. As the hub of both intermediary metabolism and xenobiotic metabolism, the liver is essential to systemic homeostasis of both lipids and glucose as well as metabolism of endogenous and exogenous toxicants. It therefore possesses highly concerted machinery for sensing xenobiotics and systemic signaling molecules, and incorporating this context into programs that regulate nutrient/energy balance and nutrient outflow. From this perspective, it is not difficult to understand how a chemical could alter systemic lipid and glucose homeostasis through its direct effects on the liver; that is the concept underlying the use of metabolism-altering drugs with primary effects in the liver such as statins, fibrates, and metformin to affect systemic homeostasis of cholesterol, lipids, and glucose, respectively ${ }^{57-59}$. Toxicants which exert effects in the liver could be expected to have similar effects, depending on what combination of mechanisms they 
perturb. In addition, both intrinsic and idiosyncratic reactions to drugs or toxicants can result in the development of steatosis ${ }^{60}$, highlighting the complexity of exposure-genotype or exposuregenotype-nutrient interactions.

NAFLD is recognized as the liver manifestation of metabolic syndrome (MS), a constellation of metabolic alterations in multiple organ systems, including obesity, insulin resistance, dyslipidemia and hypertension, that appear to be connected (appear together more often than would be expected by chance). In 2004, the National Heart, Lung and Blood Institute (NHLBI) and the American Heart Association (AHA) released recommendations for diagnosis of MS with a primary outcome of cardiovascular disease. According to the Adult Treatment Panel-III (ATP-III), MS may be diagnosed by the presence of abnormalities in three or more of the following five criteria, shown in Table $4^{61}$. With similar tools but a focus on insulin-resistant diabetes as the pathological endpoint of interest, the International Diabetes Federation released similar guidelines which require obesity as well as abnormality in two of the other criteria ${ }^{62}$.

Regardless of the exact cutoffs required for diagnosis, the criteria cluster around several interrelated components: obesity, insulin resistance, dyslipidemia, and progressive cardiovascular disease. NAFLD is not currently part of the criteria for MS diagnosis, however, it is frequently found with other components of MS and the presence of NAFLD (by persistent elevations in liver enzymes ALT using the lower, gender-specific ranges defined by Prati et al., $2002^{63}$ ) had a significantly higher positive predictive value for development of insulin resistance than ATP-III in nonobese, nondiabetic subjects ${ }^{64}$. Multiple studies (reviewed in Anstee et al., 2013) indicate that the presence of NAFLD/NASH can predict the development of cardiovascular disease and T2DM after adjustment for obesity ${ }^{65}$. The significance of these findings is straightforward: the liver is an early site of dysfunction in MS, and liver injury can predict both MS and its component diseases early in the natural history of this complex syndrome.

The liver, therefore, is a central hub in the progression of MS, however, because fatty liver disease may be a cause or effect of systemic metabolic dysfunction, there may be differences in specific direct and compensatory responses of hepatocytes depending on the initiating event(s). These differences in engaged pathways may also affect the mechanisms 


\section{Table 4}

Criteria for the diagnosis of Metabolic Syndrome (MS/CVD and IR/T2DM)

\begin{tabular}{|c|c|c|c|}
\hline $\begin{array}{l}\text { Component } \\
\text { Disease }\end{array}$ & Test Parameter & $\begin{array}{l}\text { NHLBI/AHA: } \\
\text { ATP-III Criteria, CHF/CVD } \\
\text { primary outcome } \\
\text { ( } 3 \text { of the } 5 \text { required) }\end{array}$ & $\begin{array}{l}\text { IDF: } \\
\text { IR/T2DM primary outcome } \\
\text { (Visceral obesity required }+2 \text { of } \\
\text { the } 4 \text { remaining) }\end{array}$ \\
\hline Obesity & $\begin{array}{l}\text { Visceral obesity } \\
\text { (waist } \\
\text { circumference) }\end{array}$ & $\begin{array}{l}>102 \mathrm{~cm} \text { in men or } \\
>88 \mathrm{~cm} \text { in women }\end{array}$ & $\begin{array}{l}\text { For Europid, Sub-Saharan } \\
\text { African and Eastern } \\
\text { Mediterranean and Middle } \\
\text { Eastern } \\
\text { Men: } \geq 94 \mathrm{~cm} \\
\text { Women: } \geq 80 \mathrm{~cm} \\
\text { For South Asian, Chinese, } \\
\text { Ethnic south and central } \\
\text { American } \\
\text { Men: } \geq 90 \mathrm{~cm} \\
\text { Women: } \geq 80 \mathrm{~cm} \\
\text { For Japanese } \\
\text { Men } \geq 85 \mathrm{~cm} \\
\text { Women } \geq 90 \mathrm{~cm}\end{array}$ \\
\hline Hypertension & $\begin{array}{l}\text { Elevated blood } \\
\text { pressure }\end{array}$ & $>130 / 85 \mathrm{~mm} \mathrm{Hg}$ & $\begin{array}{l}>13085 \mathrm{~mm} \mathrm{Hg} \\
\text { (or treatment history) }\end{array}$ \\
\hline $\begin{array}{l}\text { Insulin } \\
\text { Resistance }\end{array}$ & $\begin{array}{l}\text { Hyperglycemia } \\
\text { (elevated fasting } \\
\text { plasma glucose) }\end{array}$ & $>6.1 \mathrm{mmol} / \mathrm{L}$ & $\geq 5.6 \mathrm{mmol} / \mathrm{l}$ \\
\hline \multirow[t]{2}{*}{$\begin{array}{l}\text { Atherogenic } \\
\text { dyslipidemia }\end{array}$} & $\begin{array}{l}\text { Elevated } \\
\text { (fasting) serum } \\
\text { triglycerides }\end{array}$ & $>1.7 \mathrm{mmol} / \mathrm{L}$ & $\begin{array}{l}\geq 1.7 \mathrm{mmol} / \mathrm{l} \\
\text { (or treatment history) }\end{array}$ \\
\hline & $\begin{array}{l}\text { Low HDL } \\
\text { cholesterol }\end{array}$ & $<1.04 \mathrm{mmol} / \mathrm{L}$ & $\begin{array}{l}<1.03 \mathrm{mmol} / \mathrm{l} \text { for men } \\
<1.29 \mathrm{mmol} / \mathrm{l} \text { for women }\end{array}$ \\
\hline
\end{tabular}

Criteria for the diagnosis of metabolic syndrome are shown. Because the endpoints of interest to the American Heart Association (column 3) and International Diabetes Foundation (column 4) differ, slightly different criteria are used to define metabolic syndrome. 
which drive changes in circulating biomarkers, including mechanistic biomarkers of hepatocyte death, transcription and release of microRNAs, and release of liver-specific functional enzymes which can be used for liver injury screening. This is important, because while liver disease occurs early in MS and can be causal, it is also generally asymptomatic, making non-invasive, low-risk detection techniques imperative.

Imaging techniques are non-invasive and low-risk and may eventually (in combination with serum biomarkers) provide sensitive and specific diagnosis and staging of fatty liver disease to subclinical populations. At present, however, ultrasonography is both subjective and qualitative, and X-ray computed tomography $(\mathrm{CT})$, proton magnetic resonance spectroscopy $\left({ }^{1} \mathrm{H}\right.$ MRS), and magnetic resonance imaging (MRI) are all resource-intensive and still cannot reliably distinguish simple steatosis from steatohepatitis ${ }^{65}$. Elevated liver transaminases are widely used clinically for diagnosis of liver injury, but they are far more effective for indicating liver injury from endogenous causes (cholestatic, vascular, or autoimmune injury) acute, severe intoxication (i.e. occupational exposures to chemicals or alcohol-induced liver injury) overdose-related or idiosyncratic drug-induced liver injury (DILI), or infectious hepatitis (Hepatitis A, B, C). TASH related to chronic organochlorine exposure may be particularly problematic, as previous research suggests that transaminase levels may remain subclinical even with severe injury ${ }^{66}$. For the purposes of environmental health research, the use of transaminases as indicators of liver injury presents an additional problem: transaminase activity is lost relatively quickly in stored blood or serum, even under optimal storage conditions ${ }^{67,68}$, reducing the ability of liver transaminase panels to reflect injury in archived samples. Emerging serum biomarkers such as microRNA 122, or the mechanistic biomarker cytokeratin 18 (CK18) may offer alternative methods to noninvasively detect subacute or chronic liver injury and liver injury of different etiologies, including TASH. CK18, because it is relatively storage-stable ${ }^{66}$ and can be detected in both whole and caspase-cleaved forms ${ }^{66,69,70}$, can discriminate hepatocyte death due to necrosis (the predominant mechanism in TASH) from hepatocellular apoptosis (the predominant mechanism in $\mathrm{ASH}, \mathrm{NASH}$, and viral hepatitis $\left.{ }^{71-73}\right)$, is a particularly appealing biomarker. 
In summary, NAFLD is recognized as a component of MS, and the functional role of the liver in both intermediary and xenobiotic metabolism suggests that progressive hepatic dysfunction could cause, contribute to, or derive from systemic metabolic dysfunction. TASH is a subcategory of NAFLD which is histologically indistinguishable from NASH and ASH but mechanistically and diagnostically unique. This difference presents an opportunity to distinguish TASH from other forms of fatty liver disease using mechanistic biomarkers, but also hinders diagnosis of liver injury due to chronic toxicant exposure because it is undetectable using typical clinical laboratory screening techniques. This is important to the field of environmental health science because exposures occurring incrementally through ingestion of contaminants are more likely to be low-level and chronic, resulting in slowly-progressive subclinical disease. 


\section{CHAPTER ONE: PCBS ARE ASSOCIATED WITH TOXICANT-ASSOCIATED STEATOHEPATITIS, INFLAMMATION, AND METABOLIC DYSREGULATION IN AN EXPOSED HUMAN POPULATION}

Anniston, Alabama was the location of a chemical manufacturing plant where an estimated 400,000 metric tons of PCBs were produced between 1929 and the $1971^{74}$. At least 20.5 metric tons of PCBs were released into the atmosphere prior to plant closure in 1971 , and nearly 19,000 metric tons of PCBs or PCB distillation residue were buried in unlined landfills near the plant site before and after production was halted ${ }^{74}$. The environment near the manufacturing plant is highly contaminated; assessment of tree bark in the area revealed PCB concentrations of $171.93 \mu \mathrm{g} / \mathrm{g}$ lipid near the plant and landfills, which dropped dramatically to $35 \mathrm{ng} / \mathrm{g}$ lipid within 17 kilometers ${ }^{75}$. In the late 1990s and early 2000s, several environmental assessments of contamination in Anniston were published, renewing concern over exposures in the residential population and the potential health effects. At that time, there existed over 6 decades of data indicating the status of PCBs as human toxicants ${ }^{6}$, although the human health effects from environmental exposure were less well characterized than industrial exposures. Nevertheless, concerns from residents, university collaborators and environmental groups initiated the Anniston Community Health Survey (ACHS) in 2003, funded by the Agency for Toxic Substances Disease Registry (ATSDR) ${ }^{76,77}$.

ACHS, and a follow-up study (ACHS-II, undertaken in 2014) are both large, crosssectional research projects which provided an opportunity to evaluate accumulation of environmental pollutants (POPs, heavy metals, etc.) in a residential population, and to assess health effects related to these exposures as well as the effects of coexposures/comorbid conditions ${ }^{49,76,78-80}$. Important findings from the ACHS include increased mean PCB levels compared to NHANES (2-3 fold), which are significantly different between non-Hispanic white and African-American/black participants ${ }^{78}$. Importantly, laboratories working with serum samples and 
data from the ACHS project have independently reported elevated prevalence of diseases related to metabolic syndrome including obesity $(54 \%)^{81}$, hypertension ${ }^{76}$, diabetes ${ }^{49}$, and dyslipidemia ${ }^{79}$. Blood pressure elevations (both in hypertensive and normotensive ranges), dyslipidemia, and diabetes (particularly in women over 55 years of age) were significantly associated with the total body burden of $\mathrm{PCBs}^{49,76,79,82}$.

As previously stated, NAFLD is the liver manifestation of metabolic syndrome, therefore, we undertook an investigation of liver disease in the Anniston cohorts. We hypothesized that biomarker-indicated liver injury would be prevalent in the ACHS and ACHS-II populations, due to the high prevalence of other metabolic disease. Because of the BMI-independent relationship between liver injury and PCB load reported in our NHANES study ${ }^{37}$ and the specific hepatocyte death mechanism detected in other studies of $\mathrm{TASH}^{66,69}$, we further hypothesized that PCBexposed individuals in Anniston would display a pattern of hepatocellular necrosis indicative of TASH. Because steatohepatitis has previously been associated with insulin resistance and systemic inflammation ${ }^{83,84}$, we anticipated that dysregulation in biomarkers of these conditions would be observed along with elevations in indicators of liver damage and death.

Our primary biomarker of hepatocyte injury was cytokeratin 18 , an intermediate filament enriched in epithelioid cells, especially hepatocytes (Figure 5). Early in apoptosis, CK-18 is cleaved by caspases, producing a neoepitope, CK18-M30. Both total CK18-M65 and the caspase-cleaved component CK18-M30 are released from dying cells and can be detected in the serum, therefore, the relative levels of these two forms can provide insight as to whether hepatocytes are undergoing predominantly apoptotic or predominantly necrotic cell death. CK18-M30 is a well-characterized biomarker of hepatocellular apoptosis. Many groups have demonstrated the utility of CK18-M30 as a diagnostic tool for steatohepatitis due to infection, alcohol, and NASH. Our lab previously demonstrated that total CK18, but not caspase-cleaved CK18, was elevated in individuals with severe liver damage due to vinyl chloride inhalation. We recognized that the effect of PCBs on liver injury could be direct or indirect (by promoting systemic metabolic dysfunction through some other mechanism or target). We 


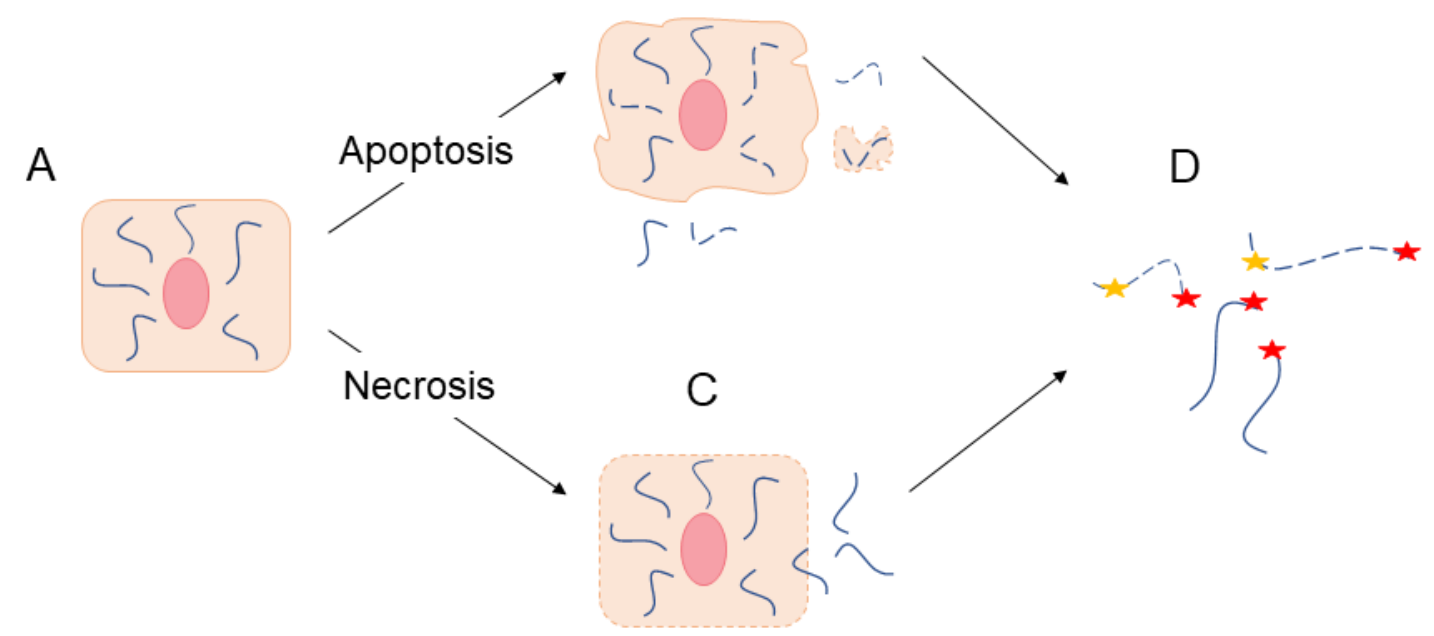

Figure 5. Origin and detection of cytokeratin 18 in serum samples

(A) Cytokeratin 18 (CK18) is an intermediate filament enriched in hepatocytes and arranged in filaments from the nuclear surface to the plasma membrane. (B) In cells undergoing apoptosis, CK18 is cleaved by caspases and released from disintegrating apoptotic bodies. (C) In cells undergoing necrosis, disintegration of the plasma membrane releases whole CK18. (D) Both cleaved and uncleaved CK18 can be detected in the serum, using an antibody against an epitope on all CK18 proteins (M65 - red stars) or an antibody against a neoepitope exposed by cleavage (M30 - yellow stars). The predominance of each form indicates the primary mechanism of hepatocyte death. Serum from individuals with NASH related to metabolic disease have a primarily apoptotic CK18 profile, while serum from individuals exposed to vinyl chloride have a primarily necrotic CK18 profile. 
hypothesized in the Anniston cohorts, a positive relationship would exist between serum concentrations of PCBs and serum levels of total CK18 due to their toxicant exposure. We hypothesized that a positive relationship between serum concentrations of PCBs and serum levels of caspase-cleaved CK18 would exist if PCBs influenced liver injury indirectly through effects on other organ systems, leading to NASH.

In addition, we evaluated the relationships between serum PCB levels and various other biomarkers of liver pathology and systemic inflammation, as well as biomarkers and calculated values reflecting metabolic function. The purpose of these evaluations was to determine the relationship (if any) between serum PCB concentration and components of the metabolic syndrome.

Lastly, because PCBs exist as a mixture, different congeners and/or groups of congeners acting on shared mechanism(s) could have differential effects, depending on the mechanisms. In the NHANES study, a significant association was found between liver injury and several individual congeners (Table 5). We hypothesized that serum levels individual congeners and congener groups would have differential associations with biomarkers of hepatocyte apoptosis and necrosis, fibrosis, insulin resistance, pancreatic function, and systemic inflammation.

To investigate our hypotheses, we determined the prevalence of TASH in both populations using the mechanistic hepatocyte death biomarker cytokeratin 18 (CK18) and other liver injury biomarkers, as well as serologic biomarkers of systemic inflammation and metabolic function (glucose and lipid metabolism). 
Table 5

PCB congeners associated with AOR for unexplained ALT elevations in NHANES 2003-2004.

\begin{tabular}{|c|c|c|c|c|c|c|}
\hline & Quartile & & & & $p$-value & \\
\hline & $1^{\text {st }}$ & $2^{\text {nd }}$ & $3^{\text {rd }}$ & $4^{\text {th }}$ & Ptrend & Ptrend-adj \\
\hline \multicolumn{7}{|c|}{ Dioxin-like PCB congeners } \\
\hline 66 & referent & $\begin{array}{l}0.6 \\
(0.2-1.5)\end{array}$ & $\begin{array}{l}2.9 \\
(1.6-5.4)\end{array}$ & $\begin{array}{l}1.9 \\
(0.9-4.0)\end{array}$ & 0.003 & 0.011 \\
\hline 74 & referent & $\begin{array}{l}2.2 \\
(1.0-5.1)\end{array}$ & $\begin{array}{l}3.0 \\
(1.5-6.0)\end{array}$ & $\begin{array}{l}6.0 \\
(2.4-14.9)\end{array}$ & $<0.001$ & 0.004 \\
\hline 105 & referent & $\begin{array}{l}1.2 \\
(0.5-3.0)\end{array}$ & $\begin{array}{l}2.8 \\
(1.2-6.5)\end{array}$ & $\begin{array}{l}3.4 \\
(1.1-10.9)\end{array}$ & 0.015 & 0.031 \\
\hline 118 & referent & $\begin{array}{l}1.8 \\
(0.7-4.8)\end{array}$ & $\begin{array}{l}3.8 \\
(1.3-11.1)\end{array}$ & $\begin{array}{l}4.4 \\
(1.4-13.7)\end{array}$ & 0.006 & 0.016 \\
\hline 126 & referent & $\begin{array}{l}1.5 \\
(0.6-3.5) \\
\end{array}$ & $\begin{array}{l}3.3 \\
(1.5-7.2) \\
\end{array}$ & $\begin{array}{l}4.3 \\
(2.0-9.4) \\
\end{array}$ & $<0.001$ & $<0.001$ \\
\hline 156 & $\begin{array}{l}1.8 \\
(0.6-5.5)\end{array}$ & $\begin{array}{l}3.4 \\
(1.3-8.7)\end{array}$ & $\begin{array}{l}5.0 \\
(1.5-17.2)\end{array}$ & $\begin{array}{l}9.4 \\
(2.5-36.2)\end{array}$ & $<.001$ & 0.004 \\
\hline 157 & $\begin{array}{l}1.5 \\
(0.8-2.8)\end{array}$ & $\begin{array}{l}4.1 \\
(1.8-9.2)\end{array}$ & $\begin{array}{l}2.1 \\
(0.6-7.6) \\
\end{array}$ & $\begin{array}{l}7.1 \\
(2.2-22.4) \\
\end{array}$ & 0.006 & 0.016 \\
\hline 167 & $\begin{array}{l}0.9 \\
(0.4-2.2)\end{array}$ & $\begin{array}{l}2.2 \\
(1.0-4.8)\end{array}$ & $\begin{array}{l}2.7 \\
(1.0-7.0)\end{array}$ & $\begin{array}{l}5.0 \\
(1.9-13.3)\end{array}$ & 0.032 & 0.011 \\
\hline 169 & $\begin{array}{l}2.4 \\
(0.9-7.0)\end{array}$ & $\begin{array}{l}3.5 \\
(1.3-9.7)\end{array}$ & $\begin{array}{l}5.0 \\
(1.8-14.0)\end{array}$ & $\begin{array}{l}2.4 \\
(0.4-12.8)\end{array}$ & 0.032 & 0.061 \\
\hline \multicolumn{7}{|c|}{ Non-dioxin-like PCB congeners } \\
\hline $138 / 158$ & referent & $\begin{array}{l}1.9 \\
(0.8-4.1)\end{array}$ & $\begin{array}{l}2.5 \\
(1.0-6.0)\end{array}$ & $\begin{array}{l}6.7 \\
(2.1-21.5)\end{array}$ & 0.001 & 0.009 \\
\hline 146 & referent & $\begin{array}{l}2.2 \\
(1.0-4.5)\end{array}$ & $\begin{array}{l}2.7 \\
(1.1-6.9)\end{array}$ & $\begin{array}{l}6.8 \\
(1.8-25.5)\end{array}$ & 0.004 & 0.019 \\
\hline 151 & $\begin{array}{l}1.1 \\
(0.3-3.6)\end{array}$ & $\begin{array}{l}0.8 \\
(0.3-2.1)\end{array}$ & $\begin{array}{l}1.0 \\
(0.4-2.3)\end{array}$ & $\begin{array}{l}2.6 \\
(1.2-5.8)\end{array}$ & 0.030 & 0.068 \\
\hline 153 & referent & $\begin{array}{l}1.5 \\
(0.6-3.6)\end{array}$ & $\begin{array}{l}2.3 \\
(0.7-7.4)\end{array}$ & $\begin{array}{l}7.2 \\
(1.7-29.9)\end{array}$ & 0.006 & 0.023 \\
\hline 170 & referent & $\begin{array}{l}2.1 \\
(1.0-4.3)\end{array}$ & $\begin{array}{l}3.1 \\
(1.1-8.7)\end{array}$ & $\begin{array}{l}4.4 \\
(1.3-14.4)\end{array}$ & 0.015 & 0.042 \\
\hline 172 & $\begin{array}{l}1.4 \\
(0.7-3.1)\end{array}$ & $\begin{array}{l}2.1 \\
(0.8-5.4)\end{array}$ & $\begin{array}{l}2.7 \\
(0.9-8.1) \\
\end{array}$ & $\begin{array}{l}3.4 \\
(1.2-9.7)\end{array}$ & 0.007 & 0.023 \\
\hline 177 & $\begin{array}{l}0.7 \\
(0.4-1.3)\end{array}$ & $\begin{array}{l}2.0 \\
(1.0-3.9)\end{array}$ & $\begin{array}{l}4.2 \\
(1.7-10.4)\end{array}$ & $\begin{array}{l}6.5 \\
(2.8-15.3)\end{array}$ & $<0.001$ & $<0.001$ \\
\hline 178 & $\begin{array}{l}1.7 \\
(0.8-3.9)\end{array}$ & $\begin{array}{l}2.1 \\
(1.0-4.6)\end{array}$ & $\begin{array}{l}4.6 \\
(1.4-15.3)\end{array}$ & $\begin{array}{l}4.8 \\
(1.3-17.4)\end{array}$ & 0.014 & 0.042 \\
\hline 183 & $\begin{array}{l}2.4 \\
(0.4-15.4)\end{array}$ & $\begin{array}{l}4.0 \\
(0.6-26.8)\end{array}$ & $\begin{array}{l}3.1 \\
(0.4-23.2)\end{array}$ & $\begin{array}{l}7.8 \\
(0.9-63.9)\end{array}$ & 0.017 & 0.042 \\
\hline 187 & referent & $\begin{array}{l}2.8 \\
(1.6-5.0)\end{array}$ & $\begin{array}{l}4.6 \\
(1.6-13.3)\end{array}$ & $\begin{array}{l}10.5 \\
(3.2-34.6)\end{array}$ & $<0.001$ & 0.002 \\
\hline $196 / 203$ & $\begin{array}{l}3.6 \\
(0.9-13.8)\end{array}$ & $\begin{array}{l}4.1 \\
(1.1-16.0)\end{array}$ & $\begin{array}{l}8.2 \\
(1.7-39.3)\end{array}$ & $\begin{array}{l}14.7 \\
(3.3-65.3)\end{array}$ & $<0.001$ & 0.002 \\
\hline
\end{tabular}

In the 2003-2004 NHANES study, individual PCB congeners associated with a significantly increased odds ratio for unexplained ALT elevation. The adjusted $p$-trend adjusts for age, sex, race/ethnicity, insulin resistance (HOMA-IR), income (PIR), and obesity (BMI). Adapted from Cave, et al 2010. Used with permission. 
CHAPTER ONE: MATERIALS AND METHODS

\section{Study design and recruitment of the ACHS and ACHS-II Cohorts} ACHS

The ACHS cohort was assembled in 2003 and has been previously described ${ }^{49,76}$. The two-stage sampling procedure involved, first, the random selection of 3,320 households from a list of all residences within the Anniston city limits. Residences in West Anniston, nearer to the PCB manufacturing facility, were oversampled. ACHS staff visited each of the selected residences, contacting 1,823 of the targeted households. One adult (>18 years of age) from each of 1,110 households agreed to participate and completed an exhaustive questionnaire covering demographic, family history, health history, and specific exposure- and lifestyle-relevant questions. The selected individual also completed a clinic visit, in which biometric measurements (height, weight, blood pressure) were assessed and a fasting serum sample was submitted. The overall completion rate was $61 \%$ (of targeted households).

The 738 serum samples represented in our ACHS mechanistic liver damage assessment and cyto/adipokine evaluation are comprised of adults completing both the survey and a clinic visit, in which a fasting blood sample was successfully submitted for analysis.

\section{ACHS MicroRNA subcohort}

152 ACHS samples submitted to the laboratory of Dr. Brian Chorley (US EPA) were selected from a stratified subset of the original 738 in which the above parameters were assessed (stratifications based on CK18 determined TASH/no liver disease, sex and race). 
ACHS-II

ACHS-II was designed to remedy limitations in the original ACHS study design and to provide longitudinal data on exposures and health outcomes in this population. ACHS-II is a subset of the original ACHS cohort, and was approached as an effort to recontact every surviving subject from ACHS. Individuals were recruited to the ACHS-II cohort by a multi-stage and multi-platform methodology described in detail by Birnbaum et al. ${ }^{80}$ Methods used by ACHS staff to initiate contact or determine ineligibility were: recruitment letters (to all original ACHS participants at baseline addresses) and a public meeting (advertised and covered by local media), further attempts via phone contact (to last known phone numbers of ACHS participants), Social Security Death Index searches. To determine the status and location of participants not reachable at their last listed number, study staff searched the Social Security Death Index, and a second recruitment mailing to all original and updated addresses. Through a combination of these approaches, 359 eligible participants were enrolled in ACHS-II, and of these, the blood volumes of 345 subjects were of adequate quantity for biomarker determination.

The University of Louisville Institutional Review Board approved the studies performed on archived, de-identified samples from these cohorts.

\section{Collection of samples and analysis of polychlorinated organic pollutant concentrations}

The de-identified serum samples examined in these studies were collected during the ACHS and ACHS-II studies and archived at $-80^{\circ} \mathrm{C}$. Identification and quantification of the levels of PCBs (35 ortho-substituted congeners in ACHS and 38 dioxin-like and non-dioxin-like congeners for ACHS-II) and other contaminants was determined by high-resolution gas chromatography/isotope dilution high-resolution mass spectrometry performed at the National Center for Environmental Health Laboratory of the Centers for Disease Control and Prevention (Atlanta, GA) ${ }^{49,} 76,78,79$.

Serum concentrations of contaminants were compared to biomarkers and calculated disease parameters both individually and based on several summation schemes based on 
previously reported risk assessment protocols and mechanistic studies. For ACHS, 35 orthosubstituted congeners were analyzed. In ACHS-II, 35 ortho-substituted congeners, 3 non-ortho, and several other classes of chemical contaminants were analyzed, including the polychlorinated dibenzo dioxin (PCDD) and polychlorinated dibenzofuran (PCDF) classes. Composition of these groupings by individual congener are described in Table 2. Structural/functional groupings of PCB congeners measured in ACHS-II.

\section{Measurement of steatohepatitis biomarkers}

CK18 is a structural protein enriched in hepatocytes which can be detected in both whole and caspase-cleaved forms, allowing for the differentiation of hepatocellular apoptosis (caspasecleaved CK18 or M30) from necrosis (CK18 M65). Because the hepatocellular death mechanism appears to vary by etiology of fatty liver disease ${ }^{83,84}$, the pattern of CK18 elevation has been used to differentiate TASH (predominantly necrotic, elevations in CK18 M65 alone) from other fatty liver diseases (predominantly apoptotic, elevated CK18 M30 and M65) ${ }^{69}$. Moreover, unlike transaminases, which the detectable property of enzyme activity is not retained during long-term storage ${ }^{67}$, CK18 levels are determined by immunoassay-based protein quantitation, detectable in samples archived for several decades ${ }^{66,69}$. This was an important consideration for biomarker selection, as the original ACHS samples had been archived for 7-9 years prior to our analysis. In both phases of the Anniston Community Health Survey, CK18 M65 and M30 (PEVIVA 10020 and 10010, Diapharma, Cincinnati, OH) were measured by separate enzyme-linked immunosorbent assays each using a monoclonal antibody recognizing a different CK18 epitope.

Quantifiable variations in several serum microRNA are associated with liver injury and other metabolic diseases. A subset of serum samples from the ACHS cohort representing subjects randomly selected from groups stratified by sex, race and liver disease status (TASH vs. no liver disease as determined by $\mathrm{CK}-18$ ) were evaluated for serum levels of 60 liver-associated microRNAs. These analyses were performed by firefly analysis through our collaborator Brian Chorley of the EPA. 
Liver function tests including alanine transaminase (ALT), asparagine transaminase (AST), alkaline phosphatase (ALP), bilirubin, and albumin are widely used non-invasive clinical screening tools for the assessment of liver injury (of diverse origins). Importantly, however, toxicantassociated steatohepatitis usually presents without transaminitis, in contrast to other forms of liver injury including NASH and ASH when commonly-used normal ranges are applied. A liver function panel including ALT, AST, ALP, total and direct bilirubin and albumin was carried out on ACHS-II samples at the University of Louisville Hospital Clinical Laboratory. Due to limited sample volumes and long storage times, clinical liver function tests were not performed on serum from ACHS.

\section{Evaluation of other serum biomarkers}

Fasting insulin, adipokines, and pro-inflammatory cytokines were also measured in archived samples from both phases of the Anniston Community Health Survey. Adipocytokines were measured using two separate multiplex bead arrays run on a Luminex IS100 system (EMD Millipore Corporation, Billerica, MA). The first array (HADK2MAG-61K) measured interleukin 6 (IL6), interleukin 8 (IL-8), monocyte chemotactic protein-1 (MCP-1), tumor necrosis factor a (TNFa), interleukin 1- $\beta$ (IL-1 $\beta$ ), insulin, and leptin. For the second array (HADK1MAG-61K), samples were diluted 1:400 in the provided assay buffer, and adiponectin, resistin, and total plasminogen activator inhibitor-1 (PAl-1) were measured. The homeostatic models of assessment (HOMA $)^{85}$ were used to evaluate insulin resistance (HOMA-IR) and pancreatic beta cell function (HOMA-B).

In ACHS-II, hyaluronic acid, an additional fibrosis biomarker was measured by ELISA (\#029-001, Corgenix, Inc., Broomfield, CO). Endotoxin was measured by limulus amoebocyte assay (\#50-650U, Lonza Walkersville, Inc., Walkersville, MD) and total antioxidant activity was measured by chromogenic assay (\#709001, Cayman Chemical Company, Ann Arbor, MI). Serum levels of a wide range of cytokines were provided by collaborators at ATSDR and SUNY, and were evaluated for associations with PCB exposures and TASH indicators. 


\section{Derivation of calculated values}

Substituted values for contaminant levels beyond the detectable range

Levels of individual congeners reported by the ATSDR laboratory as "undetectable" were substituted with a value equal to the lower limit of detection for the specific congener divided by the square root of $2^{86}$. When measured concentrations below the stated lower limit of detection were reported from the laboratory, those measurements (rather than substituted values) were used ${ }^{78}$. This method was previously employed to analyze and report associations between serum PCB level and diabetes ${ }^{49}$ and hypertension ${ }^{76}$ in the ACHS cohort.

Substituted values for serum biomarker levels beyond the detectable range

Biomarkers assessed in our laboratory which were below the level of detection (IL-1 $\beta<1.3$ pg/ml; IL-6 < 0.96 pg/ml; insulin < 9.6 pg/ml; IL-8 < 0.64 pg/ml; MCP-1 < 1.3 pg/ml; TNFa < 0.64 $\mathrm{pg} / \mathrm{ml}$ ) were set to half the lower limit of detection. Leptin levels greater than the upper limit of quantification (leptin $>600 \mathrm{ng} / \mathrm{ml}, 1$ record) were set to $600 \mathrm{ng} / \mathrm{ml}$.

\section{Total Lipids}

Values for total lipids were calculated by a standard ${ }^{87}$ which has been used previously to report findings in the ACHS cohort ${ }^{49}, 76$. The formula for total lipids is listed in Figure 3, Equation 7.

\section{Lipid-adjusted PCB}

PCBs are highly lipophilic and move between physiological compartments along with lipids. Therefore, adjustment for the total lipid content of serum is used to normalize total PCB concentrations in serum, and PCBs are expressed PCB wet weight per gram lipid.

\section{HOMA-IR and HOMA-B}

The homeostatic model of assessment uses weighted fasting serum glucose and insulin measurements to assess adequacy of compensation mechanisms: insulin secretion in response to glucose load and glucose disposal in response to insulin secretion. Together, they are models 
used to determine pancreatic dysfunction vs. insulin resistance as etiologies in hyperglycemia ${ }^{85,88}$. The results are dimensionless and represent insulin resistance and percent pancreatic function. Formulae for calculating HOMA parameters are listed in Figure 3, Equations 5 and 6.

PCB groupings and TEQ

PCBs have been grouped in various ways by structure and function for purposes of risk assessment and association studies. We incorporated several of these techniques into our analyses. Because only 35 ortho PCBs were quantified in serum samples from the ACHS cohort, for purposes of evaluating significant associations with our measured parameters, we presented the data as lipid-adjusted serum levels of individual congeners and lipid-adjusted levels of the sum of all 35 congeners measured ( $\Sigma$ PCB-O). In ACHS-II, the panel of PCBs and other contaminants was expanded, increasing the number of dioxin-like compounds measured. In this group, we were therefore able to present associations between measured parameters and the lipid-adjusted serum levels of individual congeners and various summations listed in Table 2. Structural/functional groupings of PCB congeners measured in ACHS-II.

As discussed in the introduction, historic focus on toxicity mechanisms dependent on AhR led to the adoption of the toxic equivalency, which is a measure of potency with regards to AhR normalized to the effects of the AhR ligand TCDD. Toxic equivalency factors (TEFs) have been established for some dioxin-like chemicals ${ }^{31}$. Besides PCBs, other chemical contributors to the total dioxin equivalency (Total Dioxin TEQ) that were measured in ACHS2 included polychlorinated dibenzo-p-dioxins (PCDDs, $n=7$ ), polychlorinated dibenzofurans (PCDFs, $n=10$ ) and polybrominated diphenyl ethers (PBDEs, $n=12$ ). Using the 2005 World Health Organization TEFs, we established toxic equivalency (TEQ) values for each dioxin-like congener, as well as TEQ by class (PCBs, PCDDs, PCDFs) and Total Dioxin TEQ for each sample within the ACHS-II cohort to explore the relationship between AhR activation and biomarker/disease status. TEQs for each congener were derived by multiplying the concentration in each sample by the TEF, and summation of TEQ by class and for all dioxins was carried out by the formulae listed in Figure 3, Equations 13 and 4 , respectively. 


\section{Statistical Analysis}

Stratification into liver disease categories

For each cohort, subjects were stratified into three groups based solely on the combination of CK18 M65 and M30 levels: one group without evident liver disease (no liver disease, or NLD, $M 30<200 \mathrm{U} / \mathrm{L}$ and $M 65<300 \mathrm{U} / \mathrm{L}$ ) and two groups with liver disease. The two liver disease groups differ by hepatocyte death mechanism: primarily necrotic hepatocyte death characteristic of TASH (M30<200 U/L and M65>300 U/L), and primarily apoptotic hepatocyte death encompassing other forms of liver disease (other liver disease, or OLD, M30>200 U/L). These cutoff values were based on prior studies ${ }^{69,89}$.

Associations between liver disease category and demographic groups or biomarkers

Counts and percentages for the main predictors were determined in the entire ACHS population and for each stratification based on liver disease category. Differences in means and frequencies by liver disease category were tested with a one-way ANOVA or chi-square test, respectively. The TASH and OLD liver disease categories were always compared against NLD. For each biomarker, regression lines were plotted within each category of liver disease status to generate a $\beta$ coefficient describing the relationship between biomarker and status. Biomarkers were analyzed with multivariable generalized linear models to assess the relationship between serum PCB levels and each outcome including with or without confounders. Unless specified elsewhere, PCB levels were adjusted for log-transformed lipid levels.

Using SAS version 9.4 (SAS Institute, Cary, NC), and a statistical significance level of 0.05 , Multinomial logistic regressions models were constructed to analyze the associations of PCB, BMI, and an interaction between PCB levels and BMI adjusted with and without additional confounders. Biomarkers, PCB levels, and total lipid levels were log-transformed. All models used wet-weight (ng/g serum) log-transformed individual PCB congeners with total lipids (also log-transformed) as

a covariate. Unless noted elsewhere, all analyses were adjusted for age (years; continuous), body mass index (BMI, $\mathrm{kg} / \mathrm{m}^{2}$; continuous), gender (male vs. female), race (African-American vs. nonHispanic white), diabetes status (none, pre-diabetic, or diabetic), alcohol use and lipid levels. 
CHAPTER ONE: RESULTS

\section{Liver injury is prevalent and persistent in a human population highly exposed to environmentally-acquired PCBs}

Summaries of population demographics for the three cohorts described are provided in Tables 6-12. In ACHS, the 738-subject cohort was comprised of a high percentage of AfricanAmericans $(46.8 \%)$, females $(70.1 \%)$, subjects $\geq 50$ years old $(63.0 \%)$, non-drinkers $(70.6 \%)$, and non-smokers $(69.0 \%)$. Most subjects $(80.3 \%)$ were either overweight $\left(\mathrm{BMI} \geq 25 \mathrm{~kg} / \mathrm{m}^{2}\right)$ or obese $\left(\mathrm{BMI} \geq 30 \mathrm{~kg} / \mathrm{m}^{2}\right)$. Subjects were categorized by liver disease status based on elevated CK18 levels consistent with either hepatocellular necrosis (TASH) - elevated CK18-M65 without CK18-M30 elevation, or apoptosis (OLD) with elevated CK18-M30. The prevalence of liver disease was $60.2 \%$ including 48.6\% with TASH and 11.5\% with Other. Higher CK18 M65 levels were found in nonHispanic whites compared to African-Americans $(p<0.001)$. A univariate sensitivity analysis excluding thirteen subjects with total wet-weight PCB levels beyond four standard deviations of the mean was performed (Table 13). This analysis showed that CK18 M65 was higher in non-Hispanic whites $(p<0.001)$ and in males $(p=0.03)$; while CK18 M30 was higher in non-Hispanic whites $(p<0.001)$ and decreased with age $(p=0.01)$.

In ACHS-II, the 345-subject cohort was comprised of a high percentage of AfricanAmericans (48.7\%), females (73.0\%), and subjects $\geq 50$ years old $(86.1 \%)$. As in ACHS, most of the subjects were non-smokers (79.4\%), however, more of the participants in ACHS-II consumed alcohol $(60.0 \%)$, with $51.3 \%$ drinking within defined limits and $8.7 \%$ drinking more than defined limits. Most subjects $(80.0 \%)$ were either overweight $(25.5 \%)$ or obese $(51.3 \%)$ by the criteria listed above. Prevalence of liver disease in this cohort was $62.0 \%: 46 \%$ with TASH and $16 \%$ with OLD. Participants with liver disease were more likely to be younger $(p=0.03)$ and white $(p=0.01)$. 
Table 6

Demographics in ACHS by Liver Disease Status - continuous variables

\begin{tabular}{|c|c|c|c|c|c|}
\hline \multirow[b]{2}{*}{ Characteristic } & \multicolumn{3}{|c|}{ Liver disease status } & \multirow[b]{2}{*}{ P-value } & \multirow[b]{2}{*}{$\begin{array}{c}\text { Total } \\
(\mathrm{n}=738)\end{array}$} \\
\hline & $\begin{array}{c}\text { None } \\
(n=294)\end{array}$ & $\begin{array}{c}\text { TASH } \\
(\mathrm{n}=359) \\
\end{array}$ & $\begin{array}{c}\text { Other } \\
(n=85)\end{array}$ & & \\
\hline Age (years) & $54.1 \pm 15.7$ & $56.0 \pm 16.3^{a}$ & $51.5 \pm 15.1$ & 0.04 & $54.7 \pm 15.9$ \\
\hline $\mathrm{BMI}\left(\mathrm{kg} / \mathrm{m}^{2}\right)$ & $31.5 \pm 7.8$ & $30.9 \pm 7.7$ & $32.1 \pm 7.7$ & 0.34 & $31.25 \pm 7.7$ \\
\hline $\begin{array}{l}\sum \text { PCBs (ortho, } \\
\text { whole weight) }\end{array}$ & $6.4 \pm 9.1$ & $7.2 \pm 14.4$ & $5.4 \pm 10.3$ & 0.40 & $6.7 \pm 12.1$ \\
\hline $\begin{array}{l}\text { Total lipids } \\
\text { (mg/dL) }\end{array}$ & $611.1 \pm 131.7$ & $643.6 \pm 163.6^{\mathrm{b}}$ & $656.9 \pm 192.4^{\mathrm{c}}$ & 0.01 & $632.2 \pm 156.3$ \\
\hline $\begin{array}{l}\text { Cytokeratin } 18 \\
\text { M65 (U/dL) }\end{array}$ & $233.6 \pm 42.6$ & $430.6 \pm 122.1^{a, b}$ & $792.5 \pm 584.9^{c}$ & $<0.001$ & $393.8 \pm 276.0$ \\
\hline $\begin{array}{l}\text { Cytokeratin } 18 \\
\text { M30 (U/dL) }\end{array}$ & $97.9 \pm 22.0$ & $124.0 \pm 28.2^{\mathrm{a}, \mathrm{b}}$ & $407.6 \pm 324.6^{c}$ & $<0.001$ & $146.3 \pm 147.1$ \\
\hline
\end{tabular}

Data are $n(\%)$ or mean \pm SD. Not all percents add to $100 \%$ due to rounding. Note that cytokeratin 18 levels were used for categorization procedures.

P-value is one-way ANOVA (means) or Pearson chi-square test, across liver disease categories.

${ }^{a}$ adj-p $<=0.05$ in pair-wise comparison of TASH vs. Other liver disease category.

${ }^{b}$ adj-p $<=0.05$ in pair-wise comparison of None vs. TASH liver disease category.

${ }^{c}$ adj- $p<=0.05$ in pair-wise comparison of None vs. Other liver disease category.

${ }^{\mathrm{d}}$ Limits are $<=30$ drinks for females and $<=60$ drinks for males. 
Table 7

Demographics in ACHS by Liver Disease Status - biometric categorical variables

\begin{tabular}{|c|c|c|c|c|c|}
\hline \multirow[b]{2}{*}{ Characteristic } & \multicolumn{3}{|c|}{ Liver disease status } & \multirow[b]{2}{*}{ P-value } & \multirow[b]{2}{*}{$\begin{array}{c}\text { Total } \\
(\mathrm{n}=738)\end{array}$} \\
\hline & $\begin{array}{c}\text { None } \\
(n=294)\end{array}$ & $\begin{array}{c}\text { TASH } \\
(n=359)\end{array}$ & $\begin{array}{c}\text { Other } \\
(n=85)\end{array}$ & & \\
\hline Gender & & & & 0.03 & \\
\hline Male & $72(24.5)$ & $123^{\mathrm{b}}(34.3)$ & $26(30.6)$ & & $221(30.0)$ \\
\hline Female & $222(75.5)$ & $236(65.7)$ & $59(69.4)$ & & $517(70.1)$ \\
\hline Race/ethnicity & & & & $<0.001$ & \\
\hline Non-Hispanic White & $117(39.8)$ & $223^{b}(62.1)$ & $53^{c}(62.4)$ & & $393(53.3)$ \\
\hline African/American & $177(60.2)$ & $136(37.9)$ & $32(37.7)$ & & $345(46.8)$ \\
\hline Age (years) & & & & 0.13 & \\
\hline$<30$ & $24(8.2)$ & $31(8.7)$ & $8(9.4)$ & & $63(8.5)$ \\
\hline $30-40$ & $41(14.0)$ & $25(7.0)$ & $12(14.1)$ & & $78(10.6)$ \\
\hline $40-50$ & $45(15.3)$ & $69(19.2)$ & $18(21.2)$ & & $132(17.9)$ \\
\hline $50-60$ & $66(22.5)$ & $75(20.9)$ & $20(23.5)$ & & $161(21.8)$ \\
\hline $60-70$ & $63(21.4)$ & $75(20.9)$ & $15(17.7)$ & & $153(20.7)$ \\
\hline$\geq 70$ & $55(18.7)$ & $84(23.4)$ & $12(14.1)$ & & $151(20.5)$ \\
\hline BMI $\left(\mathrm{kg} / \mathrm{m}^{2}\right)$ & & & & 0.40 & \\
\hline$<18.5$ & $3(1.0)$ & $2(0.6)$ & $0(0.0)$ & & $5(0.7)$ \\
\hline $18.5-24.9$ & $61(20.8)$ & $67(18.7)$ & $14(16.5)$ & & $142(19.3)$ \\
\hline $25-29.9$ & $62(21.1)$ & $109(30.4)$ & $20(23.5)$ & & $191(26.0)$ \\
\hline $30-34.9$ & $77(26.2)$ & $90(25.1)$ & $22(25.9)$ & & $189(25.7)$ \\
\hline $35-39.9$ & $48(16.3)$ & $48(13.4)$ & $15(17.7)$ & & $111(15.1)$ \\
\hline$\geq 40$ & $42(14.3)$ & $42(11.7)$ & $14(16.5)$ & & $98(13.3)$ \\
\hline Missing & $1(0.3)$ & $1(0.3)$ & $0(0.0)$ & & \\
\hline
\end{tabular}

Data are $n(\%)$ or mean \pm SD. Not all percents add to $100 \%$ due to rounding. Note that cytokeratin 18 levels were used for categorization procedures.

P-value is one-way ANOVA (means) or Pearson chi-square test, across liver disease categories.

${ }^{a}$ adj-p $<=0.05$ in pair-wise comparison of TASH vs. Other liver disease category.

${ }^{b}$ adj-p $<=0.05$ in pair-wise comparison of None vs. TASH liver disease category.

${ }^{c}$ adj- $p<=0.05$ in pair-wise comparison of None vs. Other liver disease category. 


\section{Table 8}

Demographics in ACHS by Liver Disease Status - lifestyle categorical variables

\begin{tabular}{lccccc}
\hline & \multicolumn{3}{c}{ Liver disease status } & & \\
\cline { 2 - 3 } Characteristic & $\begin{array}{c}\text { None } \\
(\mathrm{n}=294)\end{array}$ & $\begin{array}{c}\text { TASH } \\
(\mathrm{n}=359)\end{array}$ & $\begin{array}{c}\text { Other } \\
(\mathrm{n}=85)\end{array}$ & P-value & $\begin{array}{c}\text { Total } \\
(\mathrm{n}=738)\end{array}$ \\
\hline Number of drinks in last 30 days & & & 0.70 & \\
$\quad$ No drinks & $205(69.7)$ & $254(70.8)$ & $62(72.9)$ & & $521(70.6)$ \\
$\quad$ Within defined limits ${ }^{\mathrm{d}}$ & $63(21.4)$ & $73(20.3)$ & $14(16.5)$ & & $150(20.3)$ \\
$\quad$ More than limit & $16(5.4)$ & $23(6.4)$ & $8(9.4)$ & & $47(6.4)$ \\
$\quad$ Missing & $10(3.4)$ & $9(2.5)$ & $1(1.2)$ & & $20(2.7)$ \\
Current Smoker & $208(70.8)$ & $243(67.7)$ & $58(68.2)$ & & $509(69.0)$ \\
$\quad$ No & $86(29.3)$ & $116(32.3)$ & $27(31.8)$ & & $229(31.0)$ \\
$\quad$ Yes & & & & 0.25 & \\
West Anniston resident & $43(14.6)$ & $62(17.3)$ & $12(14.1)$ & & $117(15.9)$ \\
$\quad$ No & $251(85.4)$ & $297(82.7)$ & $73(85.9)$ & & $621(84.2)$ \\
$\quad$ Yes &
\end{tabular}

Data are $n(\%)$ or mean \pm SD. Not all percents add to $100 \%$ due to rounding. Note that cytokeratin 18 levels were used for categorization procedures.

P-value is one-way ANOVA (means) or Pearson chi-square test, across liver disease categories.

a adj-p $<=0.05$ in pair-wise comparison of TASH vs. Other liver disease category.

${ }^{b}$ adj- $p<=0.05$ in pair-wise comparison of None vs. TASH liver disease category.

${ }^{c}$ adj-p $<=0.05$ in pair-wise comparison of None vs. Other liver disease category.

${ }^{\mathrm{d}}$ Limits are $<=30$ drinks for females and $<=60$ drinks for males. 
Table 9

Demographics in ACHS-II by Liver Disease Status - continuous variables

\begin{tabular}{|c|c|c|c|c|c|}
\hline \multirow[b]{2}{*}{ Characteristic } & \multicolumn{3}{|c|}{ Liver disease status } & \multirow[b]{2}{*}{ P-value } & \multirow[b]{2}{*}{$\begin{array}{c}\text { Total } \\
(n=345)\end{array}$} \\
\hline & $\begin{array}{c}\text { None } \\
(n=131)\end{array}$ & $\begin{array}{c}\text { TASH } \\
(n=158)\end{array}$ & $\begin{array}{c}\text { Other } \\
(n=56)\end{array}$ & & \\
\hline Age (years) & $63.8 \pm 12.7$ & $63.4 \pm 13.3$ & $59.1 \pm 12.9$ & 0.06 & $62.9 \pm 13.1$ \\
\hline BMI $\left(\mathrm{kg} / \mathrm{m}^{2}\right)$ & $32.0 \pm 9.5$ & $31.2 \pm 7.2$ & $32.2 \pm 7.2$ & 0.59 & $31.7 \pm 8.1$ \\
\hline $\begin{array}{l}\sum \text { PCBs (ortho and } \\
\text { non-ortho, whole } \\
\text { weight), ppb }\end{array}$ & $6.0 \pm 6.4$ & $5.4 \pm 7.2$ & $5.6 \pm 9.8$ & 0.20 & $5.7 \pm 7.4$ \\
\hline $\begin{array}{l}\text { Cytokeratin } 18 \text { M65 } \\
(\mathrm{U} / \mathrm{dL})\end{array}$ & $231.8 \pm 46.0$ & $436.7 \pm 142.9$ & $624.8 \pm 368.1$ & $<.001$ & $389.4 \pm 226.5$ \\
\hline $\begin{array}{l}\text { Cytokeratin } 18 \text { M30 } \\
(\mathrm{U} / \mathrm{dL})\end{array}$ & $84.1 \pm 26.5$ & $111.6 \pm 36.3$ & $380.1 \pm 269.8$ & $<.001$ & $144.7 \pm 153.1$ \\
\hline Total lipids (mg/dL) & $611.1 \pm 143.7$ & $632.5 \pm 162.1$ & $622.1 \pm 154.8$ & 0.47 & $622.7 \pm 154.0$ \\
\hline Gender & & & & 0.07 & \\
\hline Male & $26(19.9)$ & $49(31.0)$ & $18(32.1)$ & & $93(27.0)$ \\
\hline Female & $105(80.2)$ & $109(69.0)$ & $38(67.9)$ & & $252(73.0)$ \\
\hline Race/ethnicity & & & & 0.01 & \\
\hline $\begin{array}{l}\text { Non-Hispanic } \\
\text { White } \\
\text { African/ }\end{array}$ & $50(38.2)$ & $90(57.0)$ & $28(50.0)$ & & $168(48.7)$ \\
\hline American & $81(61.8)$ & $68(43.0)$ & $28(50.0)$ & & $177(51.3)$ \\
\hline
\end{tabular}

Data are $n(\%)$ or mean \pm SD. Not all percents add to $100 \%$ due to rounding.

$\mathrm{P}$-value is one-way ANOVA (means) or Pearson chi-square test, across liver disease categories.

Abbreviations: BMI, body mass index; None, no liver disease; Other, other liver disease; $\Sigma \mathrm{PCB}$, sum of polychlorinated biphenyl congeners; TASH, toxicant associated steatohepatitis 


\section{Table 10}

Demographics in ACHS-II by Liver Disease Status - biometric categorical variables

\begin{tabular}{|c|c|c|c|c|c|}
\hline \multirow[b]{2}{*}{ Characteristic } & \multicolumn{3}{|c|}{ Liver disease status } & \multirow[b]{2}{*}{ P-value } & \multirow[b]{2}{*}{$\begin{array}{c}\text { Total } \\
(\mathrm{n}=345)\end{array}$} \\
\hline & $\begin{array}{c}\text { None } \\
(n=131)\end{array}$ & $\begin{array}{c}\text { TASH } \\
(n=158)\end{array}$ & $\begin{array}{c}\text { Other } \\
(\mathrm{n}=56)\end{array}$ & & \\
\hline Gender & & & & 0.07 & \\
\hline $\begin{array}{l}\text { Male } \\
\text { Female }\end{array}$ & $\begin{array}{c}26(19.9) \\
105(80.2)\end{array}$ & $\begin{array}{c}49(31.0) \\
109(69.0)\end{array}$ & $\begin{array}{l}18(32.1) \\
38(67.9)\end{array}$ & & $\begin{array}{c}93(27.0) \\
252(73.0)\end{array}$ \\
\hline Race/ethnicity & & & & 0.01 & \\
\hline & $50(38.2)$ & $90(57.0)$ & $28(50.0)$ & Non-Hispanic & $168(48.7)$ \\
\hline African/American & $81(61.8)$ & $68(43.0)$ & $28(50.0)$ & & $177(51.3)$ \\
\hline \multicolumn{4}{|l|}{ Age (years) } & 0.03 & \\
\hline$<30$ & 0 & $3(1.9)$ & 0 & & $3(0.9)$ \\
\hline $30-40$ & $6(4.6)$ & $5(3.2)$ & $5(8.9)$ & & $16(4.6)$ \\
\hline $40-50$ & $13(9.9)$ & $11(7.0)$ & $5(8.9)$ & & $29(8.4)$ \\
\hline $50-60$ & $24(18.3)$ & $43(27.2)$ & $23(41.1)$ & & $90(26.1)$ \\
\hline $60-70$ & $42(32.0)$ & $41(26.0)$ & $10(17.9)$ & & $93(27.0)$ \\
\hline$\geq 70$ & $46(35.1)$ & $55(34.8)$ & $13(23.2)$ & & $114(33.0)$ \\
\hline \multicolumn{4}{|l|}{ BMI $\left(\mathrm{kg} / \mathrm{m}^{2}\right)$} & \multicolumn{2}{|l|}{0.73} \\
\hline$<18.5$ & $1(0.8)$ & 0 & 0 & & $1(0.3)$ \\
\hline $18.5-24.9$ & $26(20.0)$ & $32(20.8)$ & $9(16.1)$ & & $67(19.5)$ \\
\hline $25-29.9$ & $39(30)$ & $44(28.6)$ & $15(26.8)$ & & $99(28.8)$ \\
\hline $30-34.9$ & $28(21.5)$ & $33(21.4)$ & $11(19.6)$ & & $73(21.2)$ \\
\hline $35-39.9$ & $16(12.3)$ & $28(18.2)$ & $9(16.1)$ & & $54(15.7)$ \\
\hline$\geq 40$ & $20(15.4)$ & $17(11.0)$ & $12(21.4)$ & & $50(14.5)$ \\
\hline Missing & 1 & & & & 1 \\
\hline \multicolumn{6}{|c|}{$\begin{array}{l}\text { Data are } \mathrm{n}(\%) \text { or mean } \pm \text { SD. Not all percents add to } 100 \% \text { due to } \\
\text { rounding. } \\
\text { P-value is one-way ANOVA (means) or Pearson chi-square test, across liver disease } \\
\text { categories. } \\
\text { Abbreviations: BMI, body mass index; None, no liver disease; Other, other liver disease; } \\
\Sigma \mathrm{PCB} \text {, sum of polychlorinated biphenyl congeners; TASH, toxicant associated steatohepatitis }\end{array}$} \\
\hline
\end{tabular}


Table 11

Demographics in ACHS-II by Liver Disease Status - health and lifestyle categorical variables

\begin{tabular}{|c|c|c|c|c|c|}
\hline \multirow[b]{2}{*}{ Characteristic } & \multicolumn{3}{|c|}{ Liver disease status } & \multirow{2}{*}{$\begin{array}{l}\mathrm{P}- \\
\text { value }\end{array}$} & \multirow[b]{2}{*}{$\begin{array}{c}\text { Total } \\
(\mathrm{n}=345)\end{array}$} \\
\hline & $\begin{array}{c}\text { None } \\
(n=131)\end{array}$ & $\begin{array}{c}\text { TASH } \\
(\mathrm{n}=158)\end{array}$ & $\begin{array}{l}\text { Other } \\
(n=56)\end{array}$ & & \\
\hline Diabetes Status & & & & 0.19 & \\
\hline Ever Diabetic & $44(33.6)$ & $69(43.7)$ & $24(42.9)$ & & $137(39.7)$ \\
\hline Non-diabetic & $87(66.4)$ & $89(56.3)$ & $32(57.1)$ & & $208(60.3)$ \\
\hline \multicolumn{3}{|c|}{$\begin{array}{l}\text { Typical Number of Drinks in Past } 12 \\
\text { Months }\end{array}$} & & 0.43 & \\
\hline No drinks & $56(42.8)$ & $57(36.1)$ & $25(44.6)$ & & $138(40.0)$ \\
\hline Within defined limits $^{d}$ & $66(50.4)$ & $87(55.1)$ & $24(42.9)$ & & $177(51.3)$ \\
\hline More than limit & $9(6.9)$ & $14(8.9)$ & $7(12.5)$ & & $30(8.7)$ \\
\hline Current Smoker & & & & 0.88 & \\
\hline No & $105(80.8)$ & $124(78.5)$ & 44 (78.6) & & $273(79.4)$ \\
\hline Yes & $25(19.2)$ & $34(21.5)$ & $12(21.4)$ & & $71(20.6)$ \\
\hline Missing & 1 & & & & 1 \\
\hline
\end{tabular}

Data are $\mathrm{n}(\%)$ or mean \pm SD. Not all percents add to $100 \%$ due to rounding.

P-value is one-way ANOVA (means) or Pearson chi-square test, across liver disease categories.

d Limits are $<=1$ drink/day for females and $<=2$ drinks/day for males.

Abbreviations: BMI, body mass index; None, no liver disease; Other, other liver disease;

$\Sigma \mathrm{PCB}$, sum of polychlorinated biphenyl congeners; TASH, toxicant associated steatohepatitis 
Table 12

Demographic characteristics and liver disease status of ACHS microRNA subcohort

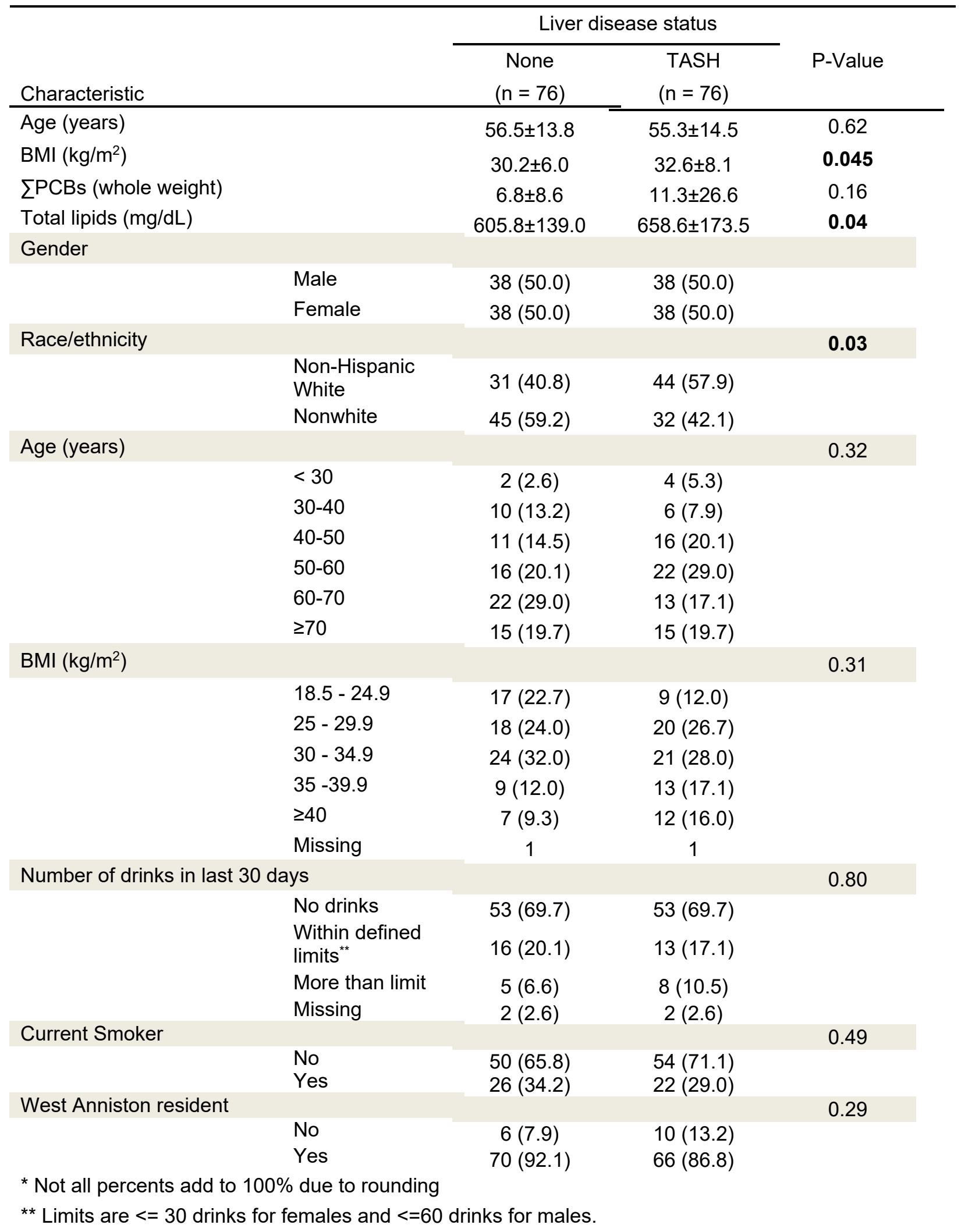




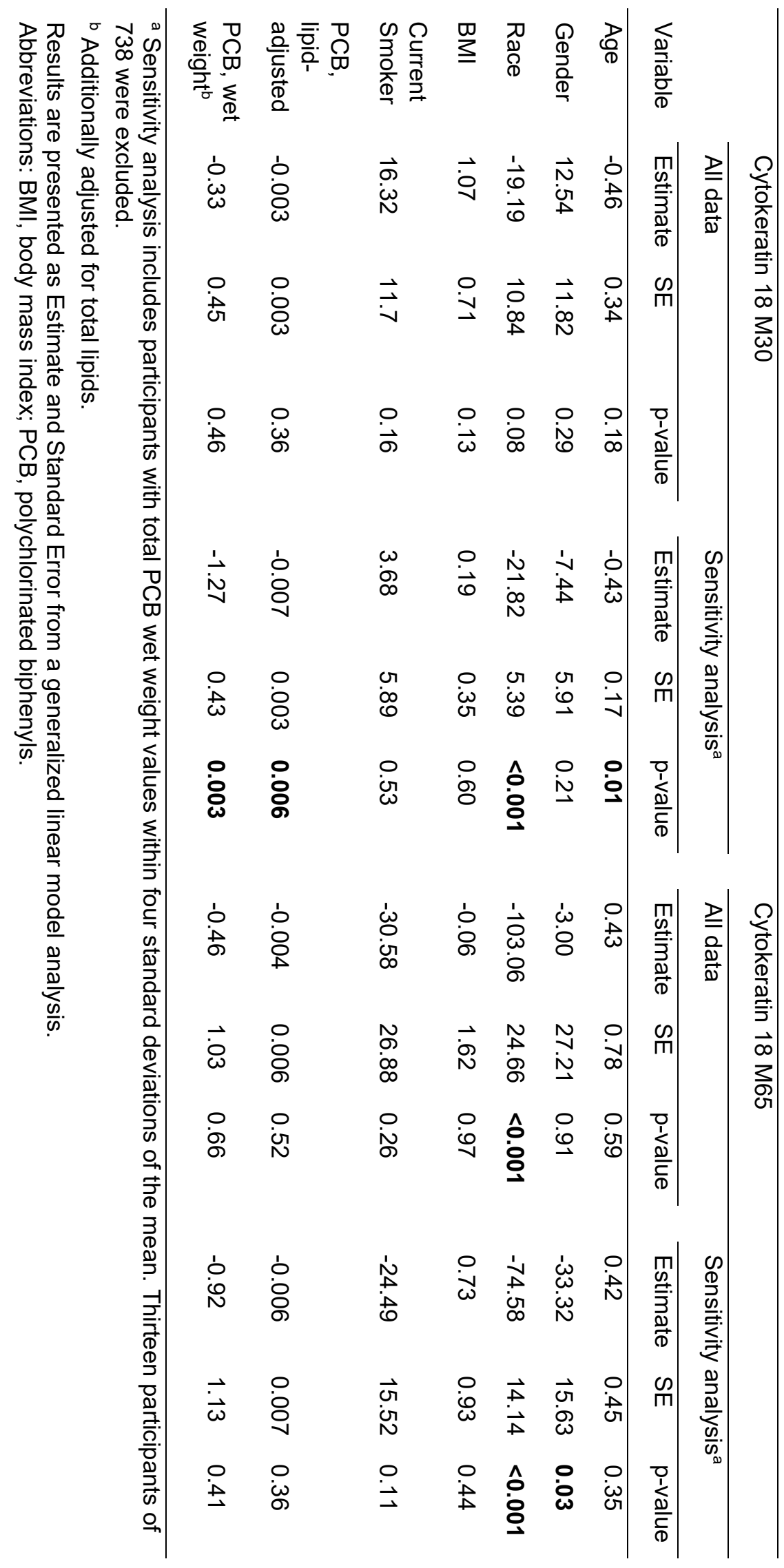

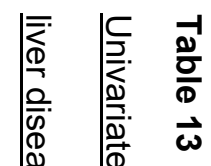

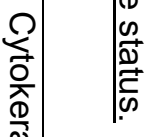


Significant demographic differences were seen across liver disease categories with respect to age, gender, and race/ethnicity. Males were more likely than females to have TASH (55.7\% vs. $45.6 \%, p<0.05)$ in ACHS, with gender differences found to be nonsignificant in ACHSII. In both studies, subjects with TASH were significantly more likely to be non-Hispanic white than subjects without liver disease (62.1\% vs. $39.8 \%$ in ACHS and $57.0 \%$ vs. $38.2 \%$ in ACHS-II). The combined prevalence of liver disease was highest among males $(67.4 \%$ in ACHS and $72.0 \%$ in ACHS-II) and non-Hispanic whites (70.2\% in both cohorts). These results are consistent with the increased susceptibility to fatty liver disease in Caucasians (compared to African Americans) and males as reported previously in other epidemiological studies ${ }^{90}$. TASH was associated with significantly increased age compared to Other in ACHS (56.0 \pm 16.3 vs. $51.5 \pm 15.1)$, but not in ACHS-II. No intragroup differences were seen in other demographic or exposure variables including total PCBs (wet weight) or West Anniston residence, body mass index, alcohol consumption, or smoking status.

Total lipids were significantly increased in both TASH and Other in ACHS (Table 6) while there were no significant intragroup differences for this parameter in ACHS-II (Table 9). Because both necrotic and apoptotic (M30) hepatocyte death contribute to total CK18 (M65), it is not surprising, based on the classification procedures, that CK18 M65 was significantly increased in both liver disease groups compared to None. However, the absolute M65 level was increased to a greater degree in Other compared to TASH, even though only M30 abnormality was used to discriminate between the two.

\section{Liver injury in Anniston is associated with increased pro-inflammatory cytokines and metabolic abnormalities.}

Steatohepatitis is associated with increased serum pro-inflammatory cytokines, which can result from and/or lead to liver injury and metabolic disease ${ }^{91,92}$. As shown in Table 14, IL-1 $\beta$, IL6, IL-8, MCP-1, PAI-1, and TNFa were all numerically higher across liver disease categories (graphs), but only MCP-1 $(p=0.01)$ and PAI-1 $(p=0.001)$ reached statistical significance in the 


\section{Table 14}

Unadjusted ACHS Biomarker levels by liver disease status.

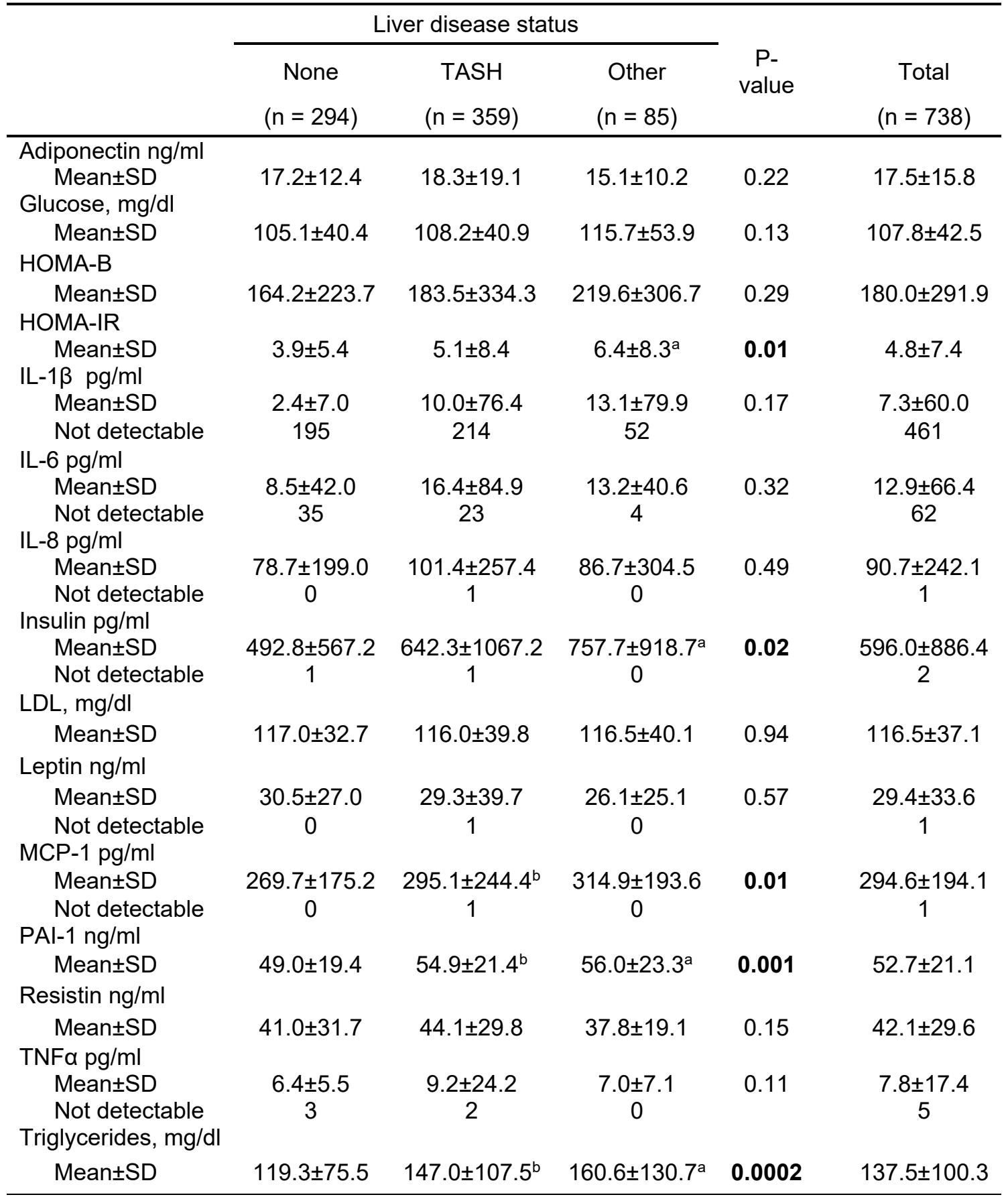

P-value is one-way ANOVA, across all liver disease categories, unadjusted.

a adj-p $<=0.05$ in pair-wise comparison of None vs. Other liver disease category.

$b$ adj-p $<=0.05$ in pair-wise comparison of None vs. TASH liver disease category.

$c$ adj- $p<=0.05$ in pair-wise comparison of TASH vs. Other liver disease category. 
unadjusted model. MCP-1 was higher in TASH than None $(295.1 \pm 244.4 \mathrm{pg} / \mathrm{ml}$ vs. $269.7 \pm 175.2$ $\mathrm{pg} / \mathrm{ml}, \mathrm{p}=0.009)$. PAl-1 was increased in both TASH $(54.9 \pm 21.4 \mathrm{ng} / \mathrm{ml}, \mathrm{p}=.001)$ and Other $(56.0 \pm 23.3 \mathrm{ng} / \mathrm{ml}, \mathrm{p}=0.02)$ compared to None $(49.0 \pm 19.4 \mathrm{ng} / \mathrm{ml})$.

NAFLD is also commonly associated with insulin resistance and hypertriglyceridemia ${ }^{93}$. The mean HOMA-IR for the overall cohort was elevated (4.8 \pm 7.4$)$ beyond one proposed cutoff for insulin resistance (HOMA-IR $>4.65$ or HOMA-IR $>3.60$ and $\left.\mathrm{BMI}>27.5 \mathrm{~kg} / \mathrm{m}^{2}\right)^{94}$. HOMA-IR varied significantly across liver disease groups $(p=0.01)$, and it was increased in Other compared to None (6.4 \pm 8.3 vs. $3.9 \pm 5.4, p=.017)$. While HOMA-IR was numerically higher in TASH $(5.1 \pm 8.4)$ than None, this trend did not reach statistical significance. Fasting insulin varied in a pattern like that observed for HOMA-IR (Table 14), and likely accounted for the observed difference in HOMA-IR as glucose levels were not different between groups. Triglycerides varied by liver disease category $(p=0.007)$ and were higher in both TASH $(147.0 \pm 107.5 \mathrm{mg} / \mathrm{dl}, \mathrm{p}=.001)$ and Other $(160.6 \pm 130.7$ $\mathrm{mg} / \mathrm{dl}, \mathrm{p}=.002)$ vs. None $(119.3 \pm 75.5 \mathrm{mg} / \mathrm{dl})$. In summary, both TASH and Other were associated with increased PAI-1 and triglycerides. MCP-1 was also increased in TASH, while both HOMA-IR and insulin were increased in Other.

In ACHS, TASH was associated with increased levels of IL-1 $\beta(p=0.04)$, IL-6 $(p=0.03)$, and PAI-1 $(p=0.03)$ in our unadjusted model (Table 15). Beta coefficients for other pro-inflammatory cytokines including IL-8, MCP-1, resistin, and TNFa were positive in TASH but did not reach statistical significance. TASH was also associated with increased HOMA-IR $(p=0.001)$ with increased pancreatic insulin production (HOMA-B, $p=0.003)$ and increased insulin levels $(p=0.01)$ (Table 16) Other was associated with increased IL-6 ( $p=0.01)$, HOMA-IR $(p=0.02)$, insulin $(p=0.04)$, and decreased leptin $(p=0.01)$. Thus, the liver disease categories remained associated with increased pro-inflammatory cytokines and hepatic insulin resistance after adjustment for confounders, although the specific cytokines varied slightly between models. This increases the certainty that the categorization procedures correctly identified liver disease. TASH appeared more pro-inflammatory than Other due to the greater number of associated cytokines. 
Table 15

$\underline{\text { Adjusted }^{\mathrm{a}} \text { beta coefficients of associations of } \Sigma \mathrm{PCBs} \text { (wet weight) and liver status with }}$ inflammatory biomarkers

\begin{tabular}{|c|c|c|c|}
\hline \multicolumn{4}{|l|}{ IL-1 $1 \beta$} \\
\hline PCB & 0.01 & 0.05 & 0.90 \\
\hline TASH vs. None & 0.18 & 0.09 & 0.04 \\
\hline Other vs. None & 0.25 & 0.14 & 0.07 \\
\hline \multicolumn{4}{|l|}{ IL-6 } \\
\hline PCB & 0.05 & 0.05 & 0.33 \\
\hline TASH vs. None & 0.27 & 0.09 & 0.003 \\
\hline Other vs. None & 0.39 & 0.14 & 0.01 \\
\hline \multicolumn{4}{|l|}{ IL-8 } \\
\hline PCB & 0.08 & 0.07 & 0.25 \\
\hline TASH vs. None & 0.15 & 0.12 & 0.20 \\
\hline Other vs. None & -0.04 & 0.18 & 0.83 \\
\hline \multicolumn{4}{|l|}{ MCP-1 } \\
\hline PCB & 0.02 & 0.03 & 0.53 \\
\hline TASH vs. None & 0.01 & 0.05 & 0.91 \\
\hline Other vs. None & -0.07 & 0.08 & 0.36 \\
\hline \multicolumn{4}{|l|}{ PAI-1 } \\
\hline PCB & -0.01 & 0.02 & 0.51 \\
\hline TASH vs. None & 0.07 & 0.03 & 0.03 \\
\hline Other vs. None & 0.07 & 0.05 & 0.15 \\
\hline \multicolumn{4}{|l|}{ TNFa } \\
\hline PCB & 0.04 & 0.03 & 0.26 \\
\hline TASH vs. None & 0.07 & 0.06 & 0.20 \\
\hline Other vs. None & -0.04 & 0.09 & 0.65 \\
\hline
\end{tabular}

${ }^{a}$ Adjustments were made for lipid levels, age, body mass index, gender, race/ethnicity, diabetes, and alcohol use. 


\section{Table 16}

Adjusted $^{\text {a }}$ beta coefficients of associations of $\Sigma P C B$ s (wet weight) and liver status with metabolic biomarkers

\begin{tabular}{|c|c|c|c|}
\hline Cytokine/Adipokine & $\beta$ & Standard Error & p-Value \\
\hline \multicolumn{4}{|l|}{ Adiponectin } \\
\hline PCB & 0.01 & 0.03 & 0.69 \\
\hline TASH vs. None & -0.04 & 0.05 & 0.43 \\
\hline Other vs. None & -0.06 & 0.08 & 0.43 \\
\hline \multicolumn{4}{|l|}{ Glucose } \\
\hline PCB & $<0.001$ & 0.01 & 1.00 \\
\hline TASH vs. None & 0.00 & 0.02 & 0.90 \\
\hline Other vs. None & 0.03 & 0.03 & 0.18 \\
\hline \multicolumn{4}{|l|}{ Insulin } \\
\hline PCB & -0.07 & 0.04 & 0.04 \\
\hline TASH vs. None & 0.22 & 0.06 & $<0.001$ \\
\hline Other vs. None & 0.19 & 0.09 & 0.04 \\
\hline \multicolumn{4}{|l|}{ HOMA-B } \\
\hline PCB & -0.07 & 0.04 & 0.08 \\
\hline TASH vs. None & 0.20 & 0.07 & 0.003 \\
\hline Other vs. None & 0.13 & 0.10 & 0.22 \\
\hline \multicolumn{4}{|l|}{ HOMA-IR } \\
\hline PCB & -0.08 & 0.04 & 0.03 \\
\hline TASH vs. None & 0.21 & 0.06 & 0.001 \\
\hline Other vs. None & 0.21 & 0.09 & 0.02 \\
\hline \multicolumn{4}{|l|}{ Leptin } \\
\hline PCB & -0.14 & 0.04 & $<0.001$ \\
\hline TASH vs. None & -0.06 & 0.06 & 0.32 \\
\hline Other vs. None & -0.24 & 0.09 & 0.01 \\
\hline \multicolumn{4}{|l|}{ Resistin } \\
\hline PCB & 0.01 & 0.03 & 0.81 \\
\hline TASH vs. None & 0.06 & 0.05 & 0.20 \\
\hline Other vs. None & -0.06 & 0.07 & 0.41 \\
\hline
\end{tabular}

${ }^{a}$ Adjustments were made for lipid levels, age, body mass index, gender, race/ethnicity, diabetes, and alcohol use. 


\section{Liver injury in Anniston was validated by microRNA analysis and clinical liver function tests}

Analysis of serum microRNA was used to validate the findings of ACHS in a subset of the cohort, described as the ACHS miRNA cohort. Demographic findings for this population are shown in Table 12. This semi-balanced subgroup differs from the other two in several demographic characteristics. TASH participants tended to have higher BMI $\left(\mathrm{kg} / \mathrm{m}^{2}: 32.6\right.$ vs. 30.2, $\left.\mathrm{p}=0.045\right)$, total lipids (mg/dL: 659 vs. 606, $p=0.04)$ and insulin/HOMA-B/HOMA-IR $(p=0.01,0.045,0.01)$. They were more likely to be non-Hispanic white $(58 \%$ vs. $41 \%, p=0.03)$. Serum levels of 68 microRNAs related to liver and other metabolic disease were quantified by Firefly assay and compared between CK18-defined TASH and NLD groups in this subcohort.

Differential levels of several microRNAs related to liver injury were seen between two liver disease categories. The well-characterized biomarker of liver injury, microRNA 122 (miR-122-5p), was significantly increased in the TASH group vs. the no liver disease (NLD) group. Several other liver- and metabolic disease-associated microRNA were different between the two groups as well, including miR-22-3p, miR-320a, and miR-375 (significantly increased), and miR-223-3p was significantly decreased in TASH vs. NLD (Table 17).

Several microRNA showed significant positive and/or negative associations with the individual isoforms of CK18, including miR-122, which was associated with an increase in CK18$\mathrm{M} 30$ and a smaller positive association with CK18-M65. Of the microRNA transcripts assayed, only miR-877-5p displayed a similar pattern. Other microRNA showed either positive (hsa_miR_22_3p, hsa_miR_320a, hsa_miR_375, or negative (hsa_miR_21_5p, hsa_miR_375, hsa_miR_92a_3p) association with CK18-M65 alone. Positive and negative associations ( $\beta$-coefficients) for microRNA vs. CK-18 isoforms are shown in Table 18.

Liver injury in ACHS-II was validated using clinical liver function tests (LFT). Clinical liver function tests including ALT, AST, Alkaline phosphatase, and total bilirubin were significantly elevated in the liver disease categories vs. control (Table 19). It is interesting to note, however, that by standard cutoffs used by the NHANES laboratory, prevalence of ALT abnormality in ACHS2 for females and males (respectively) would be $7.6 \%$ and $3.9 \%$ in the NLD category, $42 \%$ and $56 \%$ in the OLD category, and $22 \%$ and $8.2 \%$ in the TASH category, respectively. By the cutoffs 
Table 17

ACHS validation - miRNA data by CK18 liver disease group

Quantile-Normalized Data

Adjusted for age, race, bmi, $\log (10)$-total PCB, $\log (10)$-lipids, plate

\begin{tabular}{lcccccc}
\hline Probe & Fold & Adj-P & FDR & Raw-P & NLD & TASH \\
\hline hsa_miR_122_5p & 1.58 & 0.02 & 0.01 & 0.003 & 83.37 & 132.13 \\
hsa_miR_223_3p & 0.91 & 0.33 & 0.06 & 0.047 & 533.99 & 485.08 \\
hsa_miR_22_3p & 1.09 & 0.26 & 0.05 & 0.04 & 865.24 & 941.12 \\
hsa_miR_320a & 1.09 & 0.04 & 0.01 & 0.01 & 1320.94 & 1444.48 \\
hsa_miR_375 & 1.18 & 0.12 & 0.03 & 0.02 & 6.94 & 8.19 \\
hsa_miR_92a_3p & 0.93 & 0.02 & 0.01 & 0.003 & 1457.91 & 1357.75 \\
\hline
\end{tabular}

A subset of samples from ACHS Phase 1 were analyzed for levels of specific microRNA

(miRNA) species associated with liver and/or metabolic disease. Individuals with CK18-

indicated TASH (TASH) were compared to individuals with CK levels indicating no liver disease

(NLD). Included in this table are those with a significant difference between groups (raw p-

value <.05) 
Table 18

Adjusted $\beta$-coefficients of CK-18 and miRNA levels.

\begin{tabular}{|c|c|c|c|c|c|c|}
\hline \multirow[b]{2}{*}{ Probe } & \multicolumn{3}{|c|}{$\begin{array}{l}\text { CK18-M30 } \\
\text { Standard }\end{array}$} & \multicolumn{3}{|c|}{$\begin{array}{l}\text { CK18-M65 } \\
\text { Standard }\end{array}$} \\
\hline & $\beta$ & Error & Raw-P & $\beta$ & Error & Raw-P \\
\hline hsa_miR_122_5p & 1.00 & 0.33 & 0.003 & 0.20 & 0.09 & 0.02 \\
\hline hsa_miR_21_5p & 0.01 & 0.13 & 0.94 & -0.19 & 0.08 & 0.02 \\
\hline hsa_miR_22_3p & 0.09 & 0.09 & 0.31 & 0.14 & 0.05 & 0.01 \\
\hline hsa_miR_29a_3p & -0.05 & 0.08 & 0.52 & -0.10 & 0.05 & 0.06 \\
\hline hsa_miR_29c_3p & -0.10 & 0.10 & 0.36 & -0.10 & 0.06 & 0.11 \\
\hline hsa_miR_320a & 0.09 & 0.07 & 0.19 & 0.10 & 0.04 & 0.04 \\
\hline hsa_miR_375 & 0.09 & 0.15 & 0.55 & 0.20 & 0.09 & 0.03 \\
\hline hsa_miR_503_5p & -0.04 & 0.10 & 0.70 & -0.12 & 0.06 & 0.05 \\
\hline hsa_miR_877_5p & 0.20 & 0.10 & 0.04 & 0.14 & 0.06 & 0.02 \\
\hline hsa_miR_92a_3p & -0.08 & 0.05 & 0.15 & -0.09 & 0.03 & 0.003 \\
\hline
\end{tabular}

Several miRNA species showed significant positive or negative associations with either CK18 M30 level or CK18 M65 level. 
Table 19

ACHS-II validation - LFTs by CK18 liver disease group

\begin{tabular}{lcccc}
\hline \multicolumn{5}{c}{ Liver disease status } \\
\hline Characteristic & None & TASH & Other & P-value \\
\hline & $(n=131)$ & $(n=158)$ & $(n=56)$ & \\
\hline AST & $25.9 \pm 8.1$ & $29.0 \pm 12.1$ & $42.2 \pm 27.4$ & $<0.001$ \\
\hline ALT & $22.8 \pm 8.6$ & $29.6 \pm 9.8$ & $38.3 \pm 19.3$ & $<0.001$ \\
\hline Alk Phos & $85.5 \pm 22.7$ & $91.1 \pm 38.9$ & $104.7 \pm 58.0$ & 0.01 \\
\hline Albumin & $4.2 \pm 0.4$ & $4.2 \pm 0.4$ & $4.2 \pm 0.5$ & 0.85 \\
\hline Total Bilirubin & $0.36 \pm 0.16$ & $0.42 \pm 0.24$ & $0.46 \pm 0.24$ & 0.01 \\
\hline
\end{tabular}

Liver injury was validated in ACHS 2 (Phase 2) using the clinical liver injury biomarkers aspartate transaminase (AST), alanine transaminase (AST), alkaline phosphatase (Alk Phos), albumin, and total bilirubin. 
suggested in Prati et al., (Table 20) prevalence of ALT abnormality in ACHS2 for females and males (respectively) would be $61 \%$ and $15 \%$ in the NLD category, $76 \%$ and $94 \%$ in the other liver disease (OLD) category, and $82 \%$ and $33 \%$ in the TASH category. By either set of definitions, ALT performs better as a diagnostic tool for individuals with liver injury in which the predominant mechanism of cell death is apoptosis.

\section{Associations between EPCBs on Liver Disease as assessed by CK18, LFTs and miRNA 122}

Previous epidemiologic studies have demonstrated dose responses for PCBs in liver disease ${ }^{95-99}$, and animal studies have demonstrated interactions between PCBs and diet-induced obesity in steatohepatitis ${ }^{22,}{ }^{23}$. Univariate analysis was performed in the ACHS-1 cohort to determine associations between $\Sigma$ PCBs and the liver injury biomarkers used to derive the liver injury categories (Table 13). There were no significant associations between $\Sigma$ PCB exposure variables (lipid-adjusted or wet weight) and either CK18 M65 or M30 in the population overall. A sensitivity analysis was performed, excluding thirteen participants of 738 with total PCB wet weight values within four standard deviations of the mean. In this sensitivity analysis alone, significant negative associations were found between both lipid-adjusted and wet-weight serum PCB content and CK18 M30, but not M65.

Multinomial logistic regression was performed using multivariate models adjusted with or without additional confounders to investigate possible relationships between $\Sigma \mathrm{PCB}$ and BMI and liver disease status (Table 21). Liver disease status was not associated with either $\Sigma$ PCB levels or BMI whether alone or in combination. In both cohorts, there was no association between $\Sigma$ PCBs or $\Sigma \mathrm{PCB}-\mathrm{O}$ on liver disease status as determined by CK-18 (Table 22). When taken together, these results demonstrate that these two summation techniques, $\Sigma$ PCBs and $\Sigma$ PCB-O, were not associated with CK-18-indicated liver disease status.

In the ACHS microRNA subcohort, after adjustment for age and race there was no association between $\Sigma P C B-O$ and miRNA 122. Two other miRNA were significantly positively associated (hsa-let-7d-5p and hsa-miR-181d-5p) (Table 23). In ACHS-II, after adjustment, there was were no associations between $\mathrm{PPCB}-\mathrm{O}$ and clinical LFTs (data not shown). 


\section{Table 20}

Cutoff values for upper limit of normal for the clinical liver injury biomarker alanine aminotransferase (ALT)

\begin{tabular}{|c|c|c|c|}
\hline & $\begin{array}{l}(2003-2004 \\
\text { NHANES study) }\end{array}$ & $\begin{array}{l}\text { UofL upper limit } \\
\text { of reference }\end{array}$ & $\begin{array}{l}\text { Prati, } 2002 \\
\text { upper limits }\end{array}$ \\
\hline Men 18-21 & $\mathrm{ALT} \geq 37 \mathrm{IU} / \mathrm{L}$ & $\mathrm{ALT} \geq 70 \mathrm{IU} / \mathrm{L}$ & $30 \mathrm{U} / \mathrm{L}$ \\
\hline Men $>21$ & $\mathrm{ALT} \geq 48 \mathrm{IU} / \mathrm{L}$ & $\mathrm{ALT} \geq 70 \mathrm{IU} / \mathrm{L}$ & $30 \mathrm{U} / \mathrm{L}$ \\
\hline Women 18-21 & $A L T \geq 30 \mathrm{IU} / \mathrm{L}$ & $\mathrm{ALT} \geq 70 \mathrm{IU} / \mathrm{L}$ & $19 \mathrm{U} / \mathrm{L}$ \\
\hline Women >21 & $A L T \geq 31 \mathrm{IU} / \mathrm{L}$ & $\mathrm{ALT} \geq 70 \mathrm{IU} / \mathrm{L}$ & $19 \mathrm{U} / \mathrm{L}$ \\
\hline
\end{tabular}

Several cutoff values for the upper limit of normal have been proposed to define liver disease based on serum alanine aminotransferase levels. 


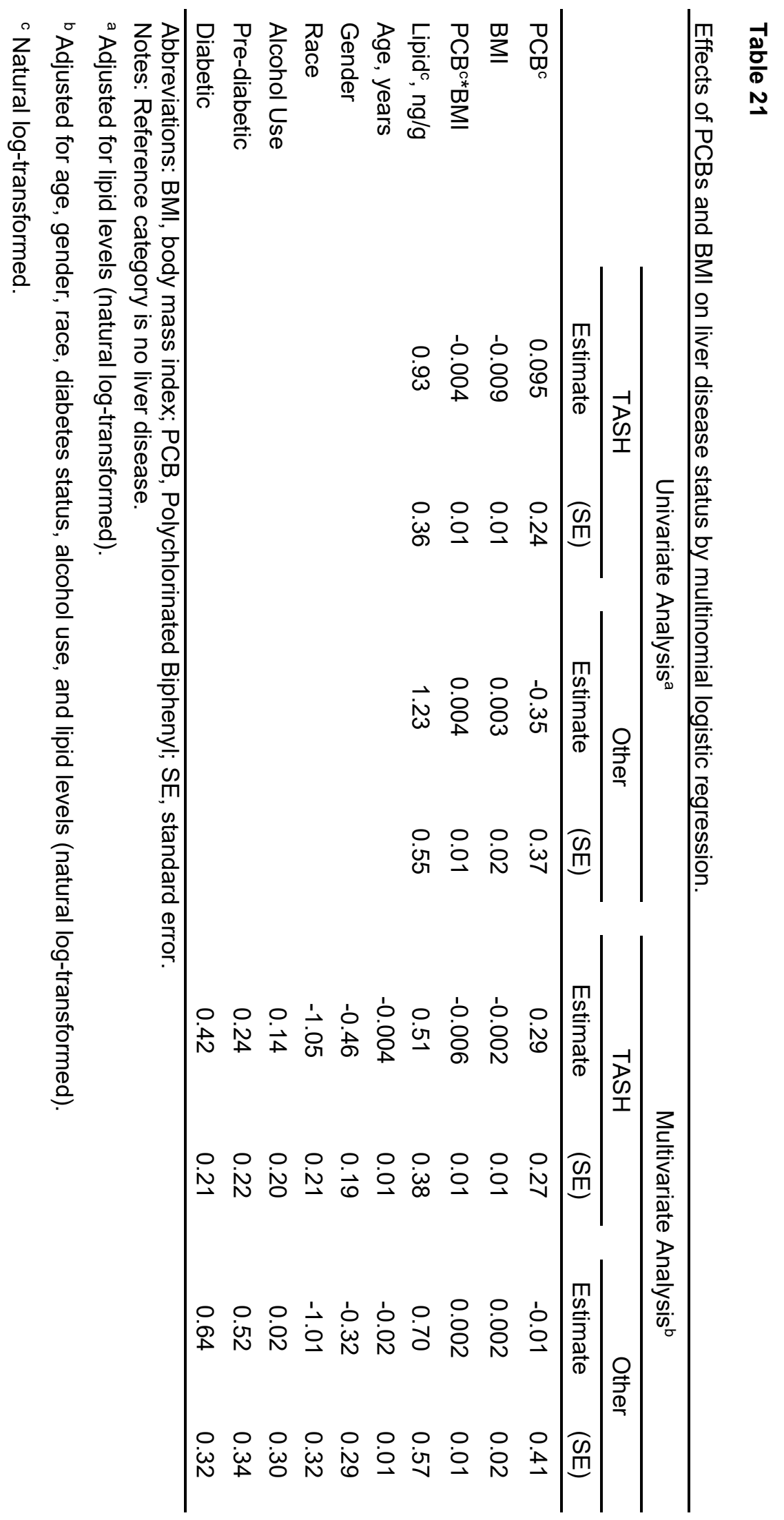




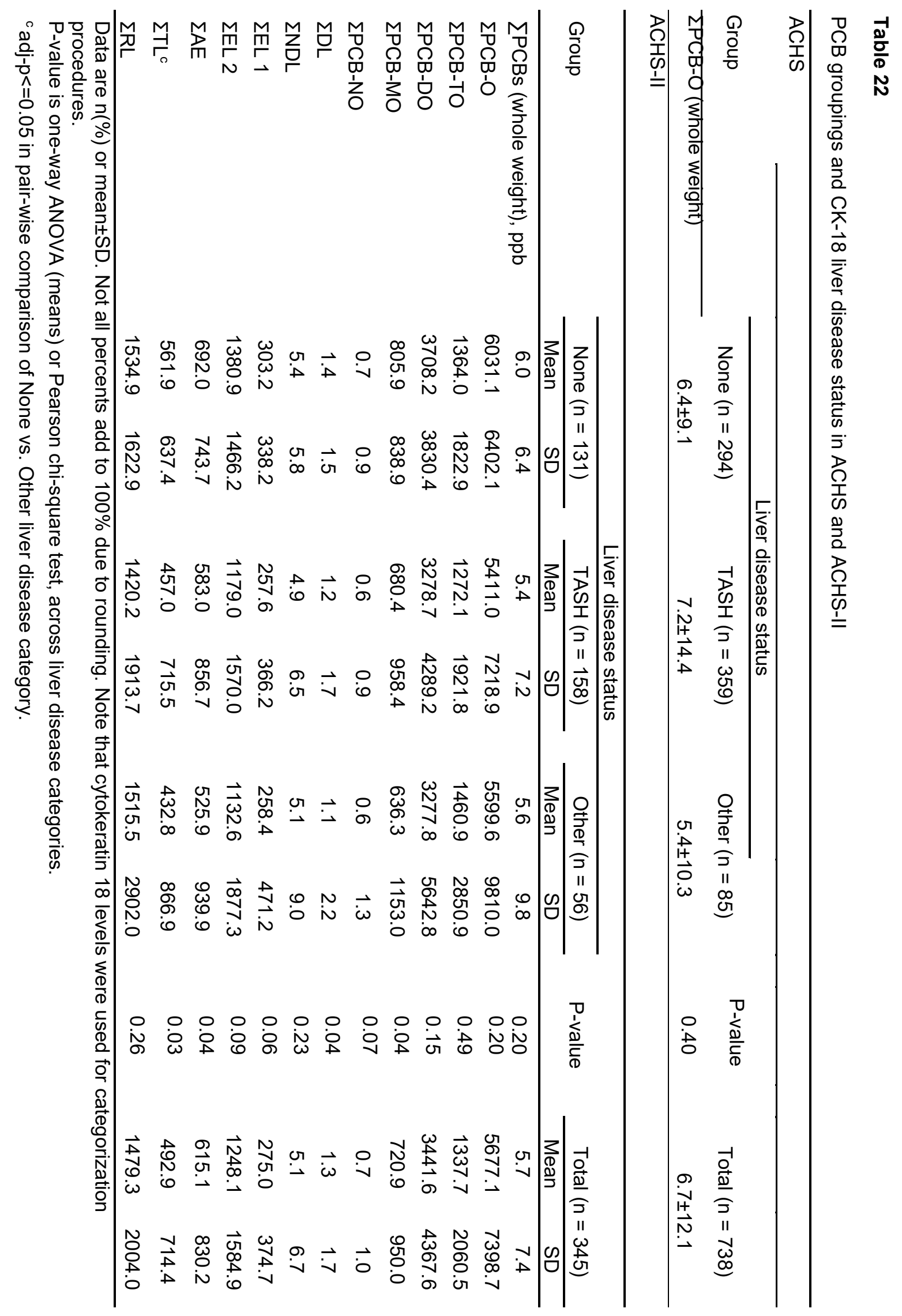


Table 23

Significant associations of $\sum$ PCBs and miRNA species

¿PCBs, whole weight

\begin{tabular}{|c|c|c|c|}
\hline miRNA species & $\beta$ & $\begin{array}{l}\text { Standard } \\
\text { Error }\end{array}$ & Raw-P \\
\hline hsa let 7d 5p & 0.07 & 0.03 & 0.03 \\
\hline hsa_miR_181d_5p & 0.08 & 0.03 & 0.003 \\
\hline
\end{tabular}

After adjustment for lipids, age, race, and inter-plate variation, no significant relationship was found between the specific liver injury biomarker miRNA-122 and $\sum$ PCBs. Two other miRNA species, miRNA-let-7d and miRNA-181d, were positively associated with $\sum$ PCBs. 


\section{Associations between individual PCB congeners and CK18-indicated liver disease in ACHS}

In the ACHS 1 cohort, TASH was positively associated with 10 PCB congeners (PCB 28, 44, 49, 52, 66 101, 110, 128, 149, 151). Three congeners associated with TASH were also associated with Other (PCB 44, 49, 52). To explore the relationship between PCB levels and cell death mechanism, we examined associations between levels of individual congeners and absolute levels of CK18 M65 and M30. Thirteen congeners were positively associated with CK18 M65, while four PCBs were positively associated with M30 (Table 24). In ACHS1, consistent with the concept that TASH is 'toxicant-associated', more PCB congeners were associated with TASH than Other (10 vs. 3). The relationships between serum PCB concentration and CK18 M65/M30 were different for individual congeners than for summed groups. Although 13 congeners were positively associated with CK18 M65, ¿PCBs was not associated with this hepatocellular necrosis biomarker. While four congeners were positively associated with M30, $\Sigma$ PCBs was inversely associated with this hepatocyte apoptosis biomarker.

In the ACHS1 population, therefore, PCBs were associated with both liver disease status and mode of hepatocyte death. Future experimental studies are required to evaluate the possible mechanistic role of PCBs in liver cell death. While animal studies have reported interactions between PCB exposures and diet-induced obesity in steatohepatitis ${ }^{22,23}$, no significant interaction was found between $\Sigma$ PCBs and BMI on liver disease status in this study. However, this analysis may have been limited by the high prevalence of overweight/obesity and the elevated PCB levels in this population.

\section{Associations between TEQs for PCB congeners and other dioxin-like species on CK18- indicated liver injury in ACHS-II}

Within the ACHS-II population, we estimated effects of dioxin-like chemicals on liver disease by using the TEQ protocol. As mentioned previously, the effects of dioxin-like chemicals have historically been attributed to AhR-mediated mechanisms, and the TEQ, by estimating the

cumulative, weighted load of AhR ligands, can be used as an exposure variable to explore the 


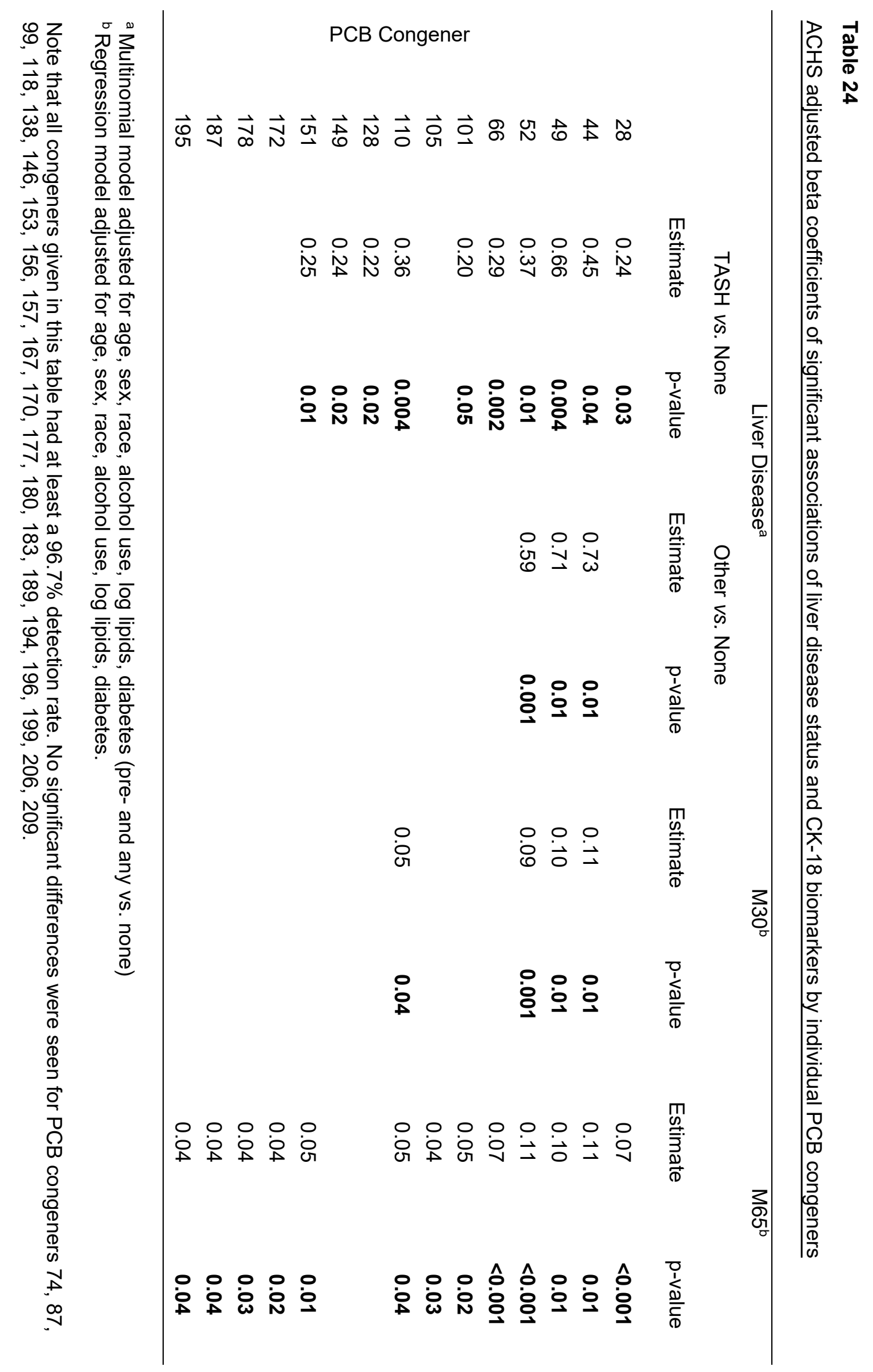


relationship between $\mathrm{AhR}$ induction and disease biomarkers. Associations between Total dioxin TEQ, TEQ by individual class (PCDD, PCDF, PCB), and TEQ for each dioxin-like chemical with a TEF available in the $2005 \mathrm{WHO}$ evaluation were evaluated and are presented in Table 25. Adjusted beta coefficients of significant associations of various TEQ summations and cytokeratin 18 biomarkers are shown in Table 26. We did not find significant associations between log transformed mean of any TEQ sum and TASH status vs. None. Non-Ortho PCB TEQ and Total Dioxins TEQ were significantly lower in the Other liver disease category compared to None.

\section{Associations between PCBs and other serum biomarkers}

PCB exposure has previously been associated with diabetes ${ }^{100}$. However, less is known regarding the potential impact of PCBs on adipocytokines. For ACHS, the effects of lipid-adjusted $\Sigma$ PCB levels on unadjusted adipocytokines and biomarkers of glucose and lipid metabolism are given as beta coefficients in Table 16. The pro-inflammatory cytokines TNFa $(p<0.001)$ and IL-6 $(p=0.001)$, were positively associated with $\Sigma P C B s$ in the unadjusted model. PCBs had no significant effects on IL-1 $\beta$, IL-8, MCP-1, PAI-1, or resistin. HOMA-B $(p<0.001)$ and insulin $(p=0.03)$ levels were inversely associated with $\Sigma$ PCB levels; while no effect was seen on HOMA-IR. Adiponectin was positively associated with PCBs $(p=0.01)$ while no difference was seen in leptin. Regression curves presented in Figure 6 graphically depict the relationships between log-transformed insulin and leptin levels with log-transformed $\Sigma$ PCBs in ACHS.

For the ACHS-2 unadjusted model, biomarkers which varied significantly with changes to serum concentrations of all PCBs measured ( $\Sigma \mathrm{PCB}), 35$ ortho-substituted congeners ( $\mathrm{PCCB}-\mathrm{O})$, or the non-dioxin-like congeners ( $\Sigma N D L)$ are shown in Table 27. In the same population and model, the biomarkers which varied significantly with changes to serum concentrations of tri/tetra-ortho substituted congeners ( $\mathrm{P} C B-T O)$, di-ortho substituted congeners ( $\mathrm{P} C B-D O)$, or mono-ortho substituted congeners ( $\mathrm{P} C \mathrm{CB}-\mathrm{MO}$ ) are shown in Table 28 , while those which varied significantly with changes to serum concentrations of non-ortho substituted congeners ( $\Sigma$ PCB-NO), or dioxinlike ( $\Sigma D L)$ are shown in Table 29. A summary of the congeners represented in each grouping is presented in Table 2. 


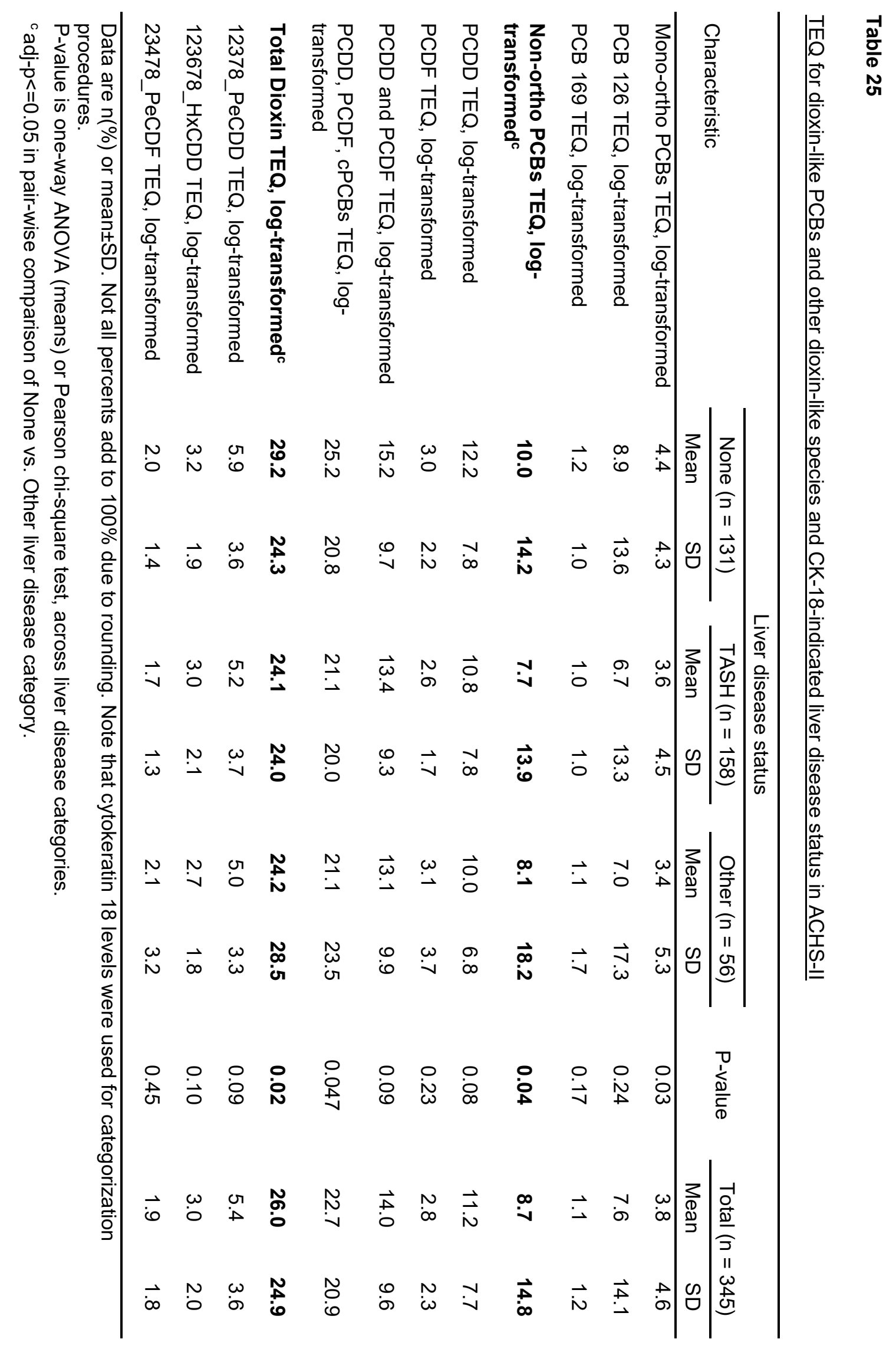




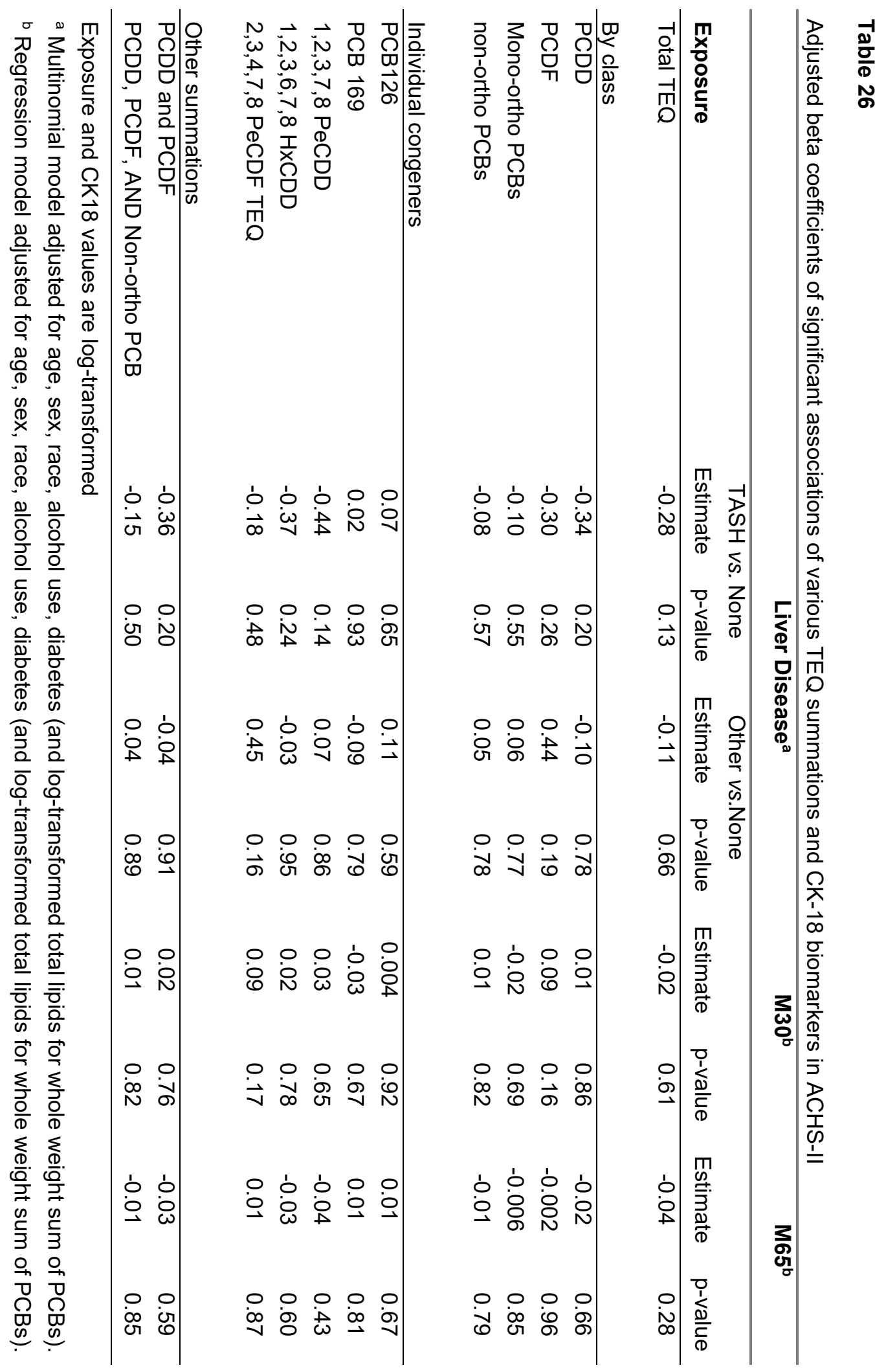



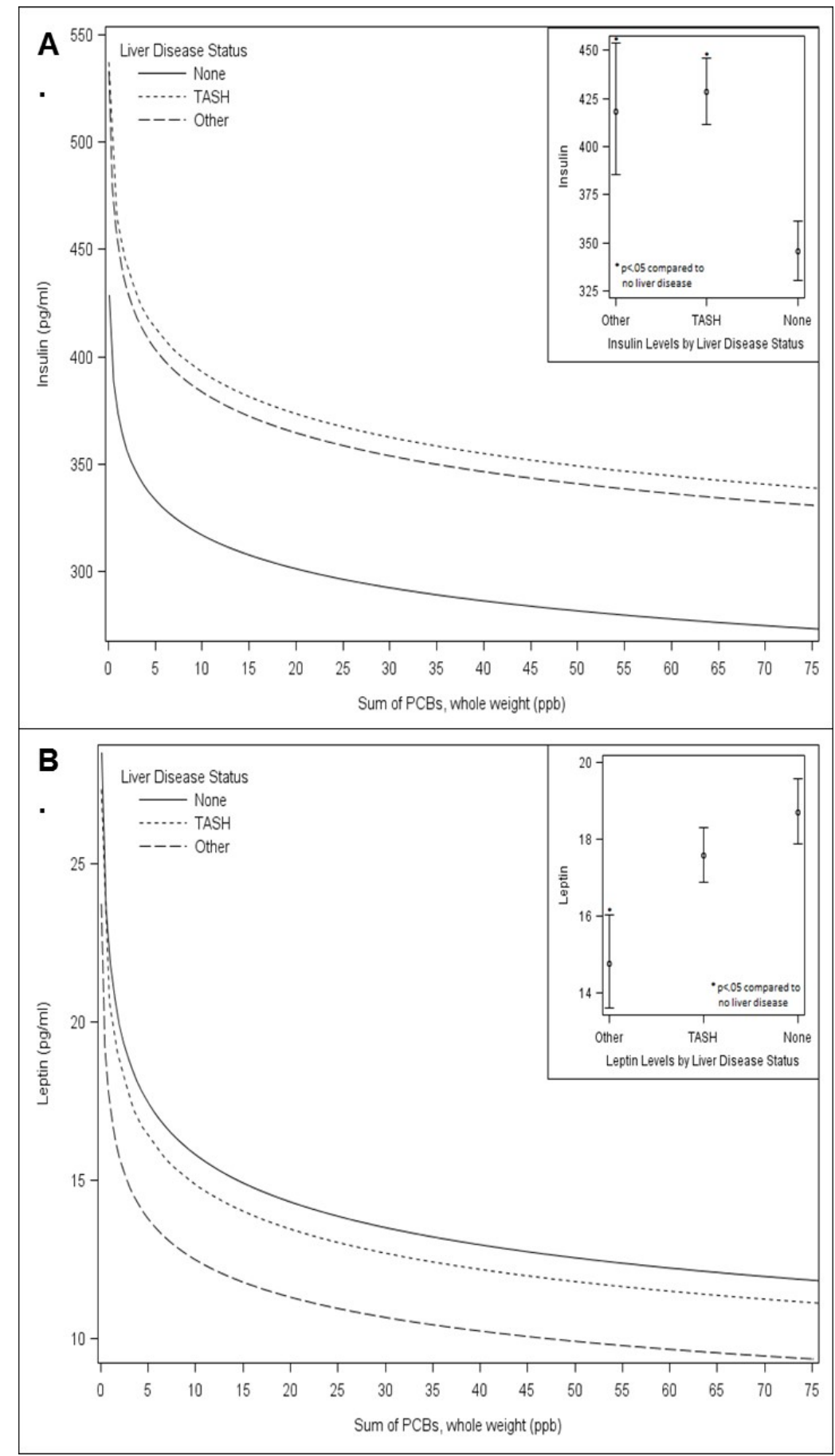

Figure 6. $\Sigma \mathrm{PCBs}$ were inversely associated with metabolic biomarkers in ACHS. Regression lines showing the change in insulin (Panel A) and leptin (Panel B) with increasing serum PCB load are shown for each liver disease group. 
Table 27

Unadjusted ACHS-II biomarker levels by sum of all PCBs ( $\sum$ PCBs ) sum of 35 orthosubstituted congeners ( $\sum$ PCB-O), and all non-dioxin-like congeners $(\Sigma N D L)$

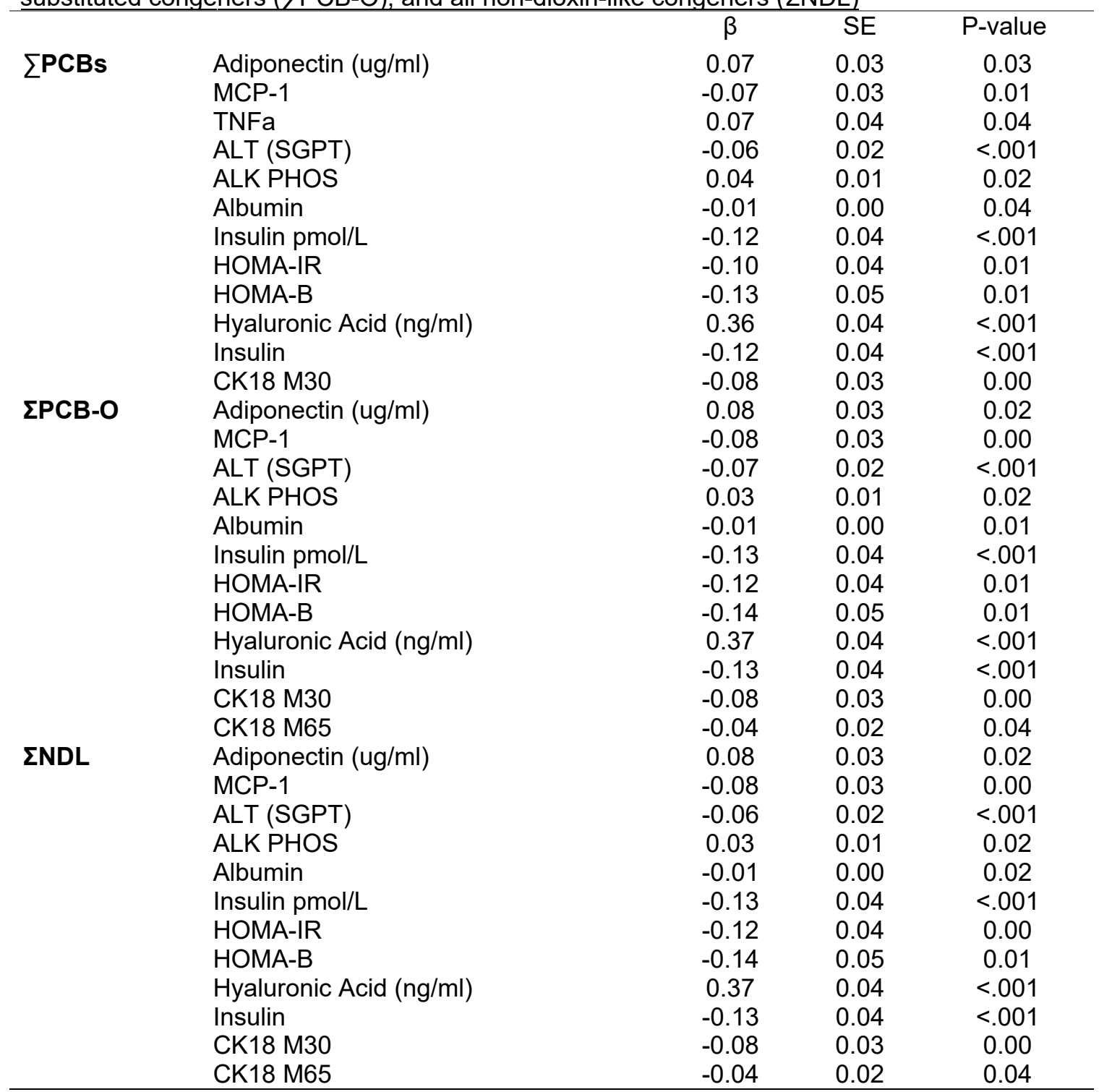

For the ACHS-II unadjusted model, biomarkers which varied significantly with

changes to serum concentrations of all PCBs measured ( $\Sigma P C B), 35$ ortho-substituted

congeners ( $\Sigma \mathrm{PCB}-\mathrm{O})$, or the non-dioxin-like congeners ( $\Sigma \mathrm{NDL})$ are shown. 
Table 28

Unadjusted ACHS-II biomarker levels by groups of ortho-substituted PCBs

\begin{tabular}{|c|c|c|c|c|}
\hline & & $\beta$ & SE & P-value \\
\hline \multirow[t]{11}{*}{ 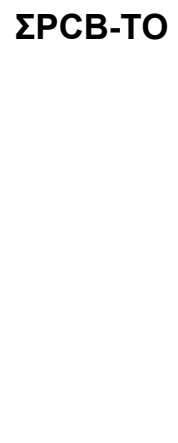 } & Adiponectin (ug/ml) & 0.08 & 0.03 & 0.01 \\
\hline & $\mathrm{MCP}-1$ & -0.08 & 0.03 & 0.00 \\
\hline & ALT (SGPT) & -0.07 & 0.02 & $<.001$ \\
\hline & ALK PHOS & 0.03 & 0.01 & 0.03 \\
\hline & Albumin & -0.01 & 0.00 & 0.01 \\
\hline & Insulin pmol/L & -0.13 & 0.03 & $<.001$ \\
\hline & HOMA-IR & -0.13 & 0.04 & 0.00 \\
\hline & HOMA-B & -0.13 & 0.05 & 0.01 \\
\hline & Hyaluronic Acid (ng/ml) & 0.34 & 0.04 & $<.001$ \\
\hline & Insulin & -0.13 & 0.03 & $<.001$ \\
\hline & CK18 M30 & -0.07 & 0.03 & 0.01 \\
\hline \multirow[t]{12}{*}{$\Sigma$ PCB-DO } & Adiponectin (ug/ml) & 0.07 & 0.03 & 0.03 \\
\hline & MCP-1 & -0.09 & 0.03 & 0.00 \\
\hline & $\mathrm{ALT}$ (SGPT) & -0.07 & 0.02 & $<.001$ \\
\hline & ALK PHOS & 0.03 & 0.01 & 0.02 \\
\hline & Albumin & -0.01 & 0.00 & 0.02 \\
\hline & Insulin pmol/L & -0.13 & 0.04 & $<.001$ \\
\hline & HOMA-IR & -0.12 & 0.04 & 0.01 \\
\hline & HOMA-B & -0.13 & 0.05 & 0.01 \\
\hline & Hyaluronic Acid (ng/ml) & 0.37 & 0.04 & $<.001$ \\
\hline & Insulin & -0.13 & 0.04 & $<.001$ \\
\hline & CK18 M30 & -0.09 & 0.03 & 0.00 \\
\hline & CK18 M65 & -0.05 & 0.02 & 0.03 \\
\hline \multirow{13}{*}{$\Sigma$ РCB-MO } & Adiponectin (ug/ml) & 0.08 & 0.03 & 0.02 \\
\hline & MCP-1 & -0.06 & 0.03 & 0.03 \\
\hline & TNFa & 0.08 & 0.04 & 0.03 \\
\hline & ALT (SGPT) & -0.08 & 0.02 & $<.001$ \\
\hline & ALK PHOS & 0.04 & 0.01 & 0.01 \\
\hline & Albumin & -0.02 & 0.00 & $<.001$ \\
\hline & Insulin pmol/L & -0.11 & 0.04 & 0.00 \\
\hline & HOMA-B & -0.14 & 0.05 & 0.01 \\
\hline & Hyaluronic Acid (ng/ml) & 0.33 & 0.04 & $<.001$ \\
\hline & Insulin & -0.11 & 0.04 & 0.00 \\
\hline & Leptin & 0.23 & 0.05 & $<.001$ \\
\hline & CK18 M30 & -0.08 & 0.03 & 0.00 \\
\hline & CK18 M65 & -0.06 & 0.02 & 0.01 \\
\hline
\end{tabular}

For the ACHS-II unadjusted model, biomarkers which varied significantly with

changes to serum concentrations of tri/tetra-ortho substituted congeners ( $\Sigma \mathrm{PCB}-\mathrm{TO}$ ), di-ortho substituted congeners ( $\Sigma$ PCB-DO), or mono-ortho substituted congeners ( $\Sigma$ PCB-MO) are shown. 
Table 29

Unadjusted ACHS-II biomarker levels by groups of non-ortho-substituted and dioxin-like PCBs

IPCB-NO

AST (SGOT)

ALT (SGPT)

Albumin

$$
\beta
$$

SE

P-value

Total Bilirubin

Direct Bilirubin

$-0.06$

0.02

0.00

Hyaluronic Acid (ng/ml)

$-0.08$

0.02

$<.001$

$-0.02$

0.01

$<.001$

$-0.07$

0.03

0.02

$-0.05$

0.02

0.04

IL-6

0.22

0.05

$<.001$

Leptin

0.14

0.06

0.01

0.27

0.06

$<.001$

CK18 M30

$-0.08$

0.03

0.02

CK18 M65

$-0.06$

0.03

0.02

TNFa

AST (SGOT)

0.09

0.03

0.01

$-0.04$

0.02

0.03

ALT (SGPT)

$-0.08$

0.02

$<.001$

ALK PHOS

0.04

0.01

0.01

Albumin

$-0.01$

0.00

$<.001$

Insulin pmol/L

$-0.10$

0.03

0.00

HOMA-B

$-0.14$

0.04

0.00

Hyaluronic Acid (ng/ml)

0.31

0.04

$<.001$

Insulin

$-0.10$

0.03

0.00

Leptin

0.18

0.05

$<.001$

CK18 M30

$-0.07$

0.02

0.00

CK18 M65

$-0.05$

0.02

0.01

For the ACHS-II unadjusted model, biomarkers which varied significantly with changes to serum concentrations of non-ortho substituted congeners ( $\Sigma \mathrm{PCB}-\mathrm{NO})$, or dioxin-like $(\Sigma \mathrm{DL})$ are shown. 
In ACHS-II, significant positive relationships were observed between $\Sigma$ PCBs and adiponectin, TNFa, alkaline phosphatase, and hyaluronic acid, while significant negative relationships were observed between $\Sigma$ PCBs and MCP-1, ALT, Albumin, Insulin, HOMA-IR, and HOMA-B. Interestingly, while most of these relationships were conserved between $\Sigma$ PCBs and other grouping structures, the positive relationship between summed PCBs and TNFa was observed only in the comparison with mono-ortho $(\beta=0.08 \pm 0.04, p=0.03)$ and dioxin-like $(\beta=0.09 \pm 0.03, p=0.01) P C B s$, along with an additional positive relationship with IL-6 $(\beta=0.14 \pm 0.06$, $p=0.01$ ) observed in the dioxin-like group (Tables 27-29). This suggests a relationship between the inflammatory processes contributing to PCB-related TASH and the structural characteristics of congeners within the exposure mixture.

In the adjusted model, $\Sigma$ PCBs remained associated with abnormal glucose metabolism. $\sum$ PCBs were associated with decreased HOMA-IR $(p=0.03)$ and insulin $(p=0.04)$, with a trend towards decreased HOMA-B ( $p=0.08)($ Table 30). $\Sigma$ PCBs were also associated with decreased leptin $(p<0.001)$. To investigate these associations further, we determined adjusted beta coefficients for individual PCB congeners and these biomarkers (Table 31). Significant inverse associations were seen between seven high molecular weight PCB congeners and both HOMA-IR and insulin. Twenty-six congeners (both low and high molecular weight) were inversely associated with leptin. Inverse associations were also found between HOMA-B and PCBs 180 and 194. $\Sigma$ PCBs was no longer associated with increased pro-inflammatory cytokines in the adjusted model. Thus, PCBs appeared to modulate both liver cell death mechanism, intermediary metabolism, and adipokines. The liver-specific impact of PCBs on inflammation was less certain due to variability between the adjusted and unadjusted models. 
Table 30

ACHS adjusted ${ }^{a}$ beta coefficients of associations of $\Sigma$ PCBs (wet weight) with biomarkers

\begin{tabular}{lccc} 
Cytokine/Adipokine & $\beta$ & $\mathrm{SE}$ & $\mathrm{p}$-Value \\
Glucose & $<0.001$ & 0.01 & 1.00 \\
Insulin & -0.07 & 0.04 & $\mathbf{0 . 0 4}$ \\
HOMA-B & -0.07 & 0.04 & 0.08 \\
HOMA-IR & -0.08 & 0.04 & $\mathbf{0 . 0 3}$ \\
IL-1 $\beta$ & 0.01 & 0.05 & 0.90 \\
IL-6 & 0.05 & 0.05 & 0.33 \\
IL-8 & 0.08 & 0.07 & 0.25 \\
Leptin & -0.14 & 0.04 & $<\mathbf{0 . 0 0 1}$ \\
MCP-1 & 0.02 & 0.03 & 0.53 \\
PAI-1 & -0.01 & 0.02 & 0.51 \\
Resistin & 0.01 & 0.03 & 0.81 \\
TNF $\alpha$ & 0.04 & 0.03 & 0.26 \\
\hline
\end{tabular}

${ }^{a}$ Adjustments were made for lipid levels, age, body mass index, gender, race/ethnicity, diabetes, and alcohol use. 
Table 31

Adjusted beta coefficients of significant associations of PCB congeners with biomarkers of glucose metabolism

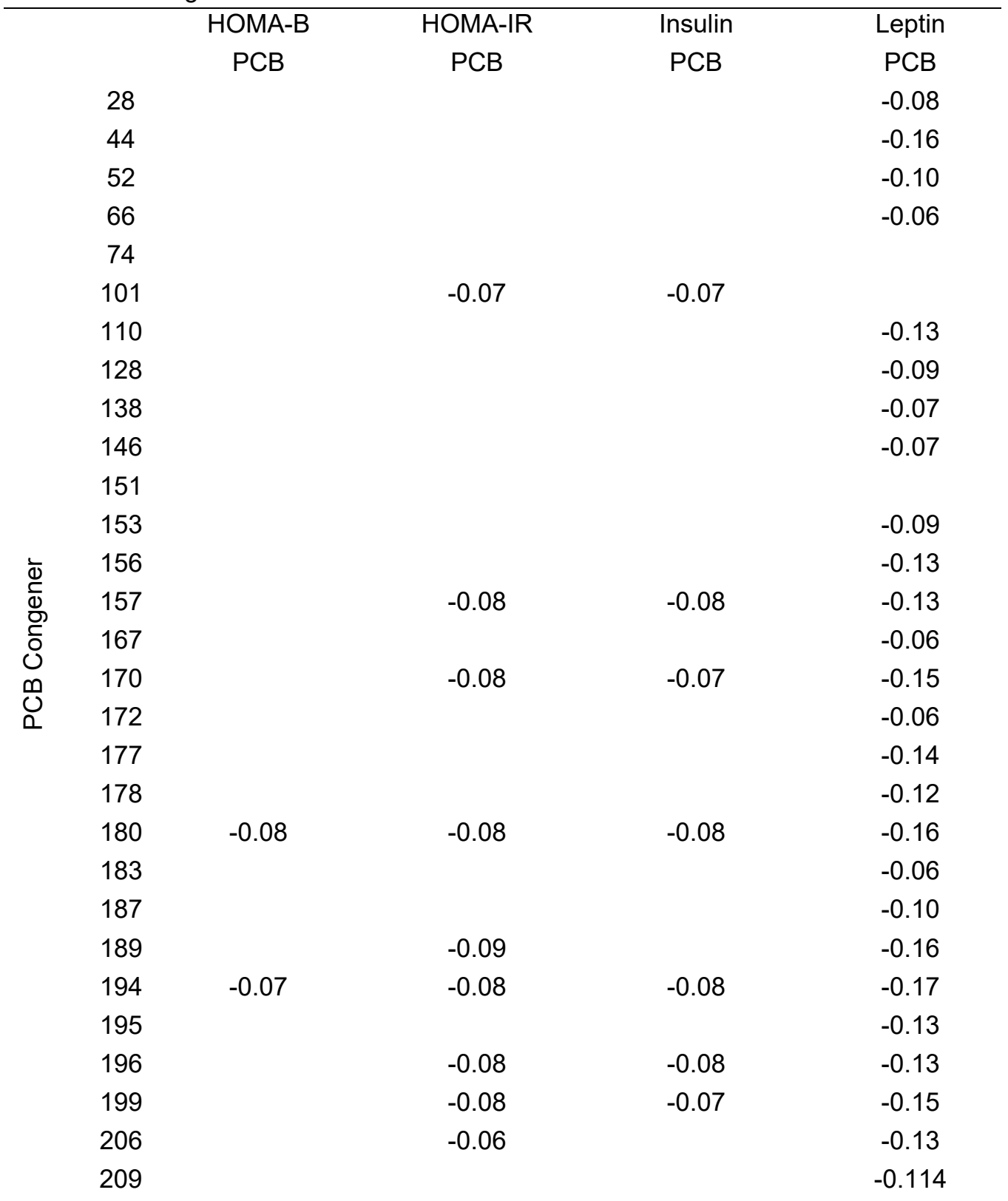

In ACHS-II, several individual congeners were associated with biomarkers or

assessments of metabolic disease. Leptin was negatively associated with most

congeners, while insulin and HOMA-IR were primarily negatively associated with higher molecular weight congeners. 


\section{CHAPTER ONE: DISCUSSION}

To our knowledge, the 738 subjects in ACHS make this the largest environmental liver disease study ever undertaken using sensitive mechanistic biomarkers. The overall liver injury prevalence of over $60 \%$ is among the highest ever reported for a residential cohort, and is well above estimates of NAFLD prevalence in the US and worldwide. Importantly, this high prevalence was maintained across both phases, suggesting that the injury remains chronic and the biomarkers of that injury are persistent. Positive associations between TASH categorization and elevated pro-inflammatory cytokines, insulin resistance, and hypertriglyceridemia further support our conclusion that chronic steatohepatitis has occurred and is persistent in these subjects.

The individuals comprising the ACHS and ACHS-II cohorts are highly contaminated with PCBs due to the proximity to a former PCB-manufacturing facility and exposure to PCBcontaining waste released into the environment. Most subjects within these cohorts were also overweight or obese, and the ACHS cohort was previously reported to have elevated prevalence of disease components of the metabolic syndrome, therefore, we expected to find increased prevalence of $\mathrm{NASH}$, consistent with these reports, and we hypothesized that mechanism of hepatic injury would be consistent with TASH because of the toxicant exposure. Liver injury in $80 \%$ of ACHS and $73.8 \%$ of ACHS-II cases was associated with hepatocellular necrosis, a characteristic of $\mathrm{TASH}^{83,84}$ but not of $\mathrm{NASH}$, or $\mathrm{ASH}$, which are associated with increased hepatocellular apoptosis ${ }^{91}$.

The liver is the principal target organ for organochlorine toxicants such as PCBs ${ }^{101-103}$. Associations between PCBs and liver injury have been demonstrated in industrial exposures and in cases of accidental poisoning in humans, and chronic PCB exposure has been shown to cause steatohepatitis in laboratory animal models. Attempts to demonstrate a straightforward 
relationship between environmental PCB exposure and liver injury in humans, however, has been frustrated, in part, by the lack of adequate biomarkers. In 2010, our laboratory published an evaluation of the 2003-2004 NHANES data, which showed an association between serum levels of PCBs and elevations of the clinical liver injury biomarker ALT. In this study, using upper limits defined in the 2003-2004 NHANES laboratory protocol ${ }^{104}, 10.6 \%$ of subjects had ALT elevation not attributable to viral hepatitis, hemochromatosis, or alcohol abuse ${ }^{37}$. Odds of inclusion in this group increased with increasing levels of serum PCBs, and inclusion was also associated with overweight/obesity and Hispanic/non-Hispanic White ethnicity, both of which are associated with increased NASH prevalence. The association between PCBs and liver injury in this population persisted even after adjustment for BMI, however, the use of ALT in the NHANES population does not allow discrimination between NASH and TASH as potential etiologies for liver injury ${ }^{37}$.

The use of CK18 M30 and M65 in the ACHS cohorts allowed dissection of these etiologies based on cell death mechanism. Our validation studies using alternative (miRNA) or approved clinical (ALT) diagnostic methods indicate significantly elevated markers of liver injury in the CK18-designated liver injury groups vs. NLD indicate that CK18 can detect liver injury in a population such as the ACHS cohorts, where TASH is suspected. This capability is particularly important when one considers the findings of the ACHS-II clinical liver function tests, which indicate statistically significant but clinically irrelevant elevations in mean ALT within the TASH group. Mean values for both NLD and "TASH" groups are well below the clinical cutoffs used in the 2003-2004 NHANES study as well as in most clinical applications. Using the upper limits recommended in Prati et al., $2002^{63}$ (shown in Table 20), which redefined the cutoffs using a cohort at low risk for chronic HCV infection or NAFLD, a much larger proportion of the ACHS-II cohort has ALT values in the abnormal range (Figure 7), however, even by the Prati definitions, ALT abnormality was only detected in $33 \%$ of males within the TASH group. This contrasts with females in the TASH group, $82 \%$ of whom had ALT abnormalities with the lower cutoff. Liver injury due to chronic TASH or TASH superimposed on NASH may not be detected in a clinical 


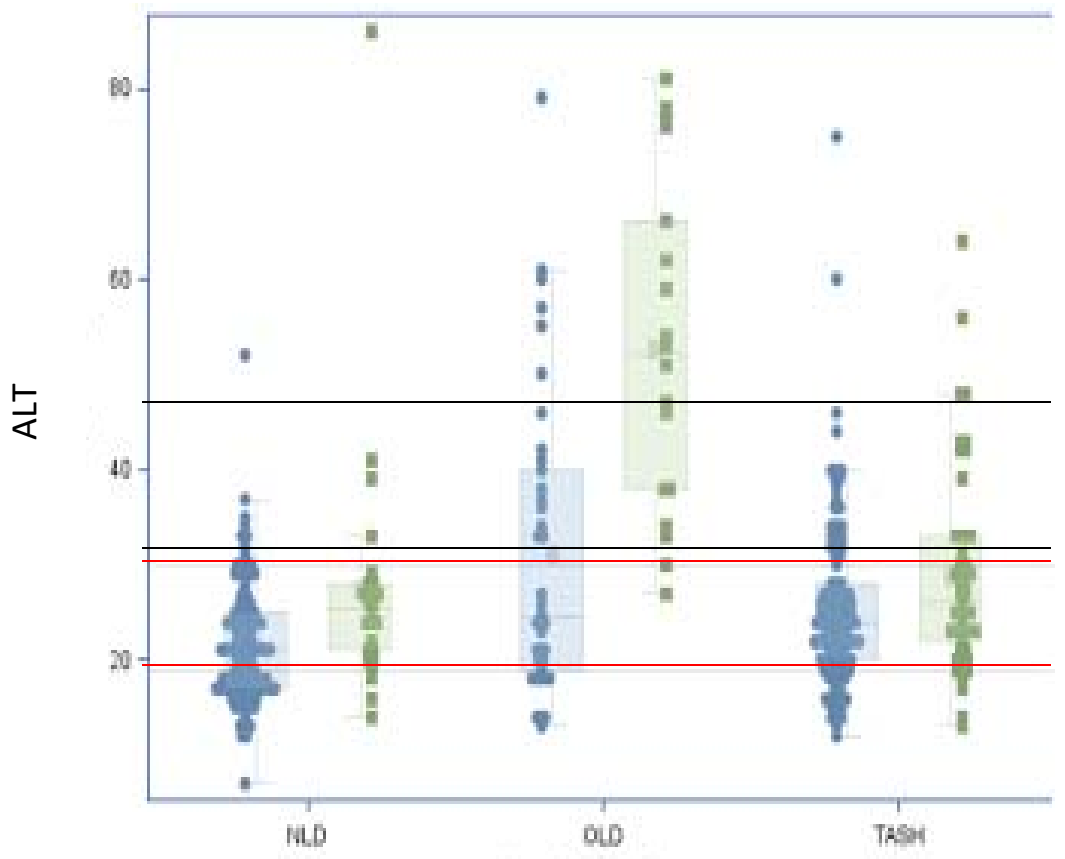

Liver Disease Status

Figure 7. Distribution of ALT levels in ACHS by liver disease category.

Distribution of ALT levels for female (blue circles) and male (green squares) participants in the three CK18-defined liver disease categories, with lines indicating upper limits of normal based on the NHANES (black lines) or Prati definition (red lines). The upper limit of normal for females is the lower line in each color, while the upper limit of normal for males is the upper line in each color. Males tended to have higher ALT levels in all groups 
setting using common screening methods, and this could delay identification therapeutic intervention.

Neither $\Sigma$ PCBs nor BMI was associated with liver disease in the ACHS cohorts, though $\Sigma \mathrm{PCBs}$ has been previously associated with liver disease in other epidemiologic studies ${ }^{37,}$ 95-99. Differences in methodologies and demographic/exposure variables might account for this discrepancy. However, exposures to 13 specific PCB congeners were associated with TASH and/or CK18 M65 elevation in the ACHS cohort. Of these, PCBs 66, 105, 149, 151, 172, 178, and 187 were previously associated with increased ALT in NHANES ${ }^{97}$. The present study using mechanistic biomarkers greatly extends this prior work. Some of the thirteen identified PCB congeners (PCBs $28,66,105$, and 128) have dioxin-like activity, while others have estrogenic $(44,49,52,101,110)$ or phenobarbital-like $(44,49,52,101,128,149,151,187,195)$ effects ${ }^{105}$. Perhaps activation of the AhR, estrogen receptor, or the constitutive androstane receptor (CAR) may be involved in PCBrelated TASH. While four congeners were associated with increased $\mathrm{CK} 18 \mathrm{M} 30$, an inverse association was observed between $\Sigma$ PCBs and this apoptosis biomarker. Notably, activation of the PCB receptor CAR, was found to be anti-apoptotic in a mouse model of cholestatic liver disease 106. This supports our clinical observation that some phenobarbital-like PCBs, which activate CAR, were associated with TASH ${ }^{106}$. Although not directly tested in this study, it is conceivable that PCBs could mediate a transition from NASH to TASH by decreasing apoptosis and promoting more proinflammatory necrotic hepatocyte death. While PCBs were a 'second hit' in the transition from dietinduced steatosis to more advanced liver disease in animal studies ${ }^{23,107-109}$, a significant interaction between PCBs and BMI on liver disease status was not observed in the present study. Perhaps the high rates of overweight/obesity and elevated PCB levels in the cohort limited the ability to detect interactions occurring at lower exposures and body weights.

In ACHS-II, the relationship between pro-inflammatory biomarkers and less orthosubstituted congener groupings again suggests receptor-mediated disease processes, although the loss of significant relationships in the model adjusted for diabetes may indicate that modulation of inflammatory response in TASH may be an extrahepatic effect. 
In ACHS, liver disease affected males (67.2\%) and non-Hispanic whites $(70.2 \%)$ disproportionately. The sex difference is consistent with a recently published report documenting increased mortality from hepatic disease in men exposed to PCB through ingestion of contaminated rice oil ${ }^{110}$. Moreover, men and Caucasians have increased susceptibility to NAFLD ${ }^{90}$. Genetic polymorphisms may contribute to ethnic differences in $\mathrm{NASH}^{90,111,112}$. For example, ethnic differences in the distribution of null alleles in the NASH susceptibility gene (patatin-like phospholipase domain containing protein 3 - PNPLA3), have been reported. We postulate that gene-environment interactions may also influence TASH; and that these interactions may have contributed to the ethnic differences observed in this study. In addition to PNPLA3, candidate genes include the pregnane $X$ receptor (PXR), which is a PCB receptor ${ }^{19}$ with at least two polymorphisms previously associated with NAFLD severity ${ }^{113}$. The observed gender differences could potentially be explained by estrogenic PCB congeners.

While steatohepatitis has long been associated abnormal adipocytokines ${ }^{83,84}$ relatively less is known about the effects of PCBs on these biomarkers. In a cohort of 992 elderly Swedish subjects, $\Sigma$ PCBs was significantly associated with vascular cell adhesion protein 1 but not IL-6, MCP-1, or TNFa ${ }^{114}$. However, Aroclor 1260 increased TNFa, IL-6, and PAI-1 ${ }^{23,108}$ in mice fed high fat diet. Adiponectin was inversely associated with PCB 28, 138, and 153 in a study of 98 Koreans 115; but $\Sigma$ PCBs was not associated with adiponectin levels in the Great Lakes Sport Fish Caught Consumers Study $(n=413)^{116}$. Leptin receptor expression was decreased in PCB-exposed children 117; and PCB-exposures induced leptin resistance in the 3T3-L1 cell culture model while increasing both TNFa and IL-6 production ${ }^{118}$; In the present study, $\Sigma$ PCBs was associated with increased TNFa and IL-6, but only in the unadjusted model. While $\Sigma$ PCBs was associated with increased adiponectin in the unadjusted model, it was associated with decreased leptin in the adjusted model. Importantly, in the adjusted model twenty-six PCB congeners were individually associated with decreased leptin. If PCBs decrease leptin expression while simultaneously inducing leptin resistance, this could be an important potential mechanism for PCB-related metabolic dysfunction.

A high prevalence of diabetes (27\%) has previously been reported in the $\mathrm{ACHS}^{49}$, and the impact of PCBs on diabetes has recently been reviewed ${ }^{100}$. In the present study, $\Sigma$ PCBs was 
associated with decreased HOMA-B in the unadjusted model with a trend $(p=0.08)$ towards decreased HOMA-B in the adjusted model. $\Sigma$ PCBs and nine individual high molecular weight congeners were associated with decreased HOMA-IR in the adjusted model. $\Sigma$ PCBs were inversely associated with insulin in both models. Adjustments were not made for diabetes medications, so it is difficult to draw firm conclusions from these data. Nonetheless, these data are by and large consistent with the findings from our recently published mouse model. In that experiment, C57BI/6 male mice fed a $42 \%$ milk fat diet for 12 weeks with or without co-exposure to a high molecular weight PCB mixture (Aroclor 1260) at a dose designed to model $\mathrm{ACHS}^{108}$. PCB treatment induced steatohepatitis, decreased HOMA-B and HOMA-IR but did not change glucose tolerance. Hepatic gluconeogenesis, glucose transporters, physical activity, food intake, and respiratory exchange rate appeared to be regulated, in part, by interactions between PCBs and the nuclear receptors $P x r$ and Car $^{108}$. These studies clearly demonstrate significant derangements in glucose metabolism occurring in the context of PCB-related liver disease. The mechanisms underpinning the complicated effects of PCBs on intermediary metabolism in steatohepatitis require further elucidation. However, based on the results of the present study, pancreatic $\beta$ cell dysfunction, rather than insulin resistance (e.g., not Type II diabetes), may be involved in the diabetes associated with PCB exposures. Importantly, such dysfunction would appear to occur in the absence of anti-islet antibodies (e.g. not Type I diabetes), suggesting the potential for Type $3 \mathrm{c}$ or pancreatogenic diabetes ${ }^{119}$ among the mechanisms of PCB-associated liver disease.

CK18 is currently the most extensively validated serum biomarker for steatohepatitis as a stand-alone test correlating with histology ${ }^{89,120}$; and the addition of transaminases to CK18 in order to create a prediction model did not improve the diagnostic value of $\mathrm{CK} 18$ alone in $\mathrm{NASH}^{89}$. In a multi-center validation study, the sensitivity and specificity of CK18 M30 for biopsy-proven NASH (vs. steatosis alone) were as high as $77 \%$ and $92 \%$ respectively depending on the test threshold 89. While CK18 correlated with ALT ${ }^{69,89}$, it offered improved diagnostic accuracy over ALT for histological $\mathrm{NASH}^{89}$ and TASH ${ }^{66}$. While CK18 is less well validated in TASH than NASH, it showed superior performance to ALT in previously published studies ${ }^{66,69}$. 
This is the first analysis of liver disease in the ACHS, and the results have several limitations. The demographic and exposure characteristics of the ACHS cohort are not representative of the overall population, limiting the generalizability of the study results. Furthermore, the ACHS cohort likely had elevated exposures to unmeasured environmental chemicals, including additional dioxin-like PCBs, most notably PCB 126. In a pilot study of 65 ACHS participants, median concentrations of the dioxin-like congeners PCB 126 and 169 approached the $95^{\text {th }}$ percentile reported for the same age group in the general U.S. population for subjects age 40-59. Importantly, dioxin-like PCBs were among the most potent environmental chemicals associated with the development of steatosis in rodent studies ${ }^{121}$, and several dioxinlike congeners were associated with TASH in ACHS. PCB exposures may also have been accompanied by exposures to dioxins, dibenzofurans, metals, and other organics related to PCB production or releases from the nearby Anniston Army Depot Superfund Site or local ferromanganese smelting operations. Although the potentially confounding effects of alcohol were taken into consideration, viral hepatitis were not excluded. Although CK18 is validated clinically, this may be the first application of CK18 in an epidemiological study. The follow-up ACHS-II study addresses some of these limitations. Additional measurements in ACHS-II will include evaluation of the relationships between $\mathrm{CK}-18$ and individual congeners. Future liver imaging and/or biopsies would help to confirm the study results.

In summary, the $60.2 \%$ prevalence of liver disease in the PCB-exposed ACHS population is among the highest ever reported for a residential cohort. Assessment of steatohepatitis in ACHS/ACHS-II population using the mechanistic liver injury biomarker CK18 revealed increased prevalence of hepatocellular necrosis, and clinical LFT analysis indicated the absence of transaminitis. This pattern is characteristic of TASH, a specific subcategory of NAFLD associated with chemical exposures. The observed hepatocellular necrosis was associated with elevations in the pro-inflammatory cytokine IL-6 and metabolic abnormalities, also consistent with TASH. Assessment of the relationship between liver injury biomarkers and PCB exposure in this population indicated a relationship between total PCB load and biomarkers of metabolic dysfunction and fibrosis, however, increasing total PCB load was not associated with increases in 
the liver injury biomarker CK18. Interestingly, positive relationships with pro-inflammatory biomarkers IL-6 and TNFa were only seen with non-ortho and dioxin-like congener groupings. Exposures to ten individual PCB congeners were also associated with TASH. This suggests that congener structures within the mixture may contribute to TASH in different ways. Individuals in this cohort are exposed to PCBs at a level 2-3 times higher than the general U.S. population, and, importantly, acquire PCBs through environmental (rather than industrial) exposure.

We therefore conclude that environmental PCB exposure is associated with increased liver injury. Individuals with TASH had corresponding elevations in other serum liver injury biomarkers, including miR-122, as well as indications of increased fibrosis and systemic inflammation. In the context of clinical hepatology practice, our findings suggest that a greater focus on the contribution of environmental chemical diseases is warranted, and the lack of correlation between clinical liver biomarkers and CK-18 and miR-122 findings suggests that standard screening practices may be insufficient to diagnose liver injury in some chemicalexposed populations. 


\section{CHAPTER TWO: PCBS INDUCE DIFFERENTIAL TRANSCRIPTION IN LIVER TISSUE FROM A MOUSE MODEL OF CHRONIC PCB/DIETARY COEXPOSURE}

The implied mechanistic differences between obesity-related NASH and TASH suggest that widespread exposure to persistent organic pollutants may contribute to liver injury. Importantly, both dioxin-like and non-dioxin-like congeners appear to drive this difference, making it clear that an understanding of the underlying mechanism is needed make therapeutic decisions and to revise risk assessment in populations exposed to related chemicals. The apparent multisystem effects of PCB exposure observed in the Anniston studies appear to converge on liver injury, but as PCB exposure in Anniston occurred in a complex coexposure environment, in the context of this epidemiological study, it is not straightforward to disentangle PCB effects from diet or metabolic disruption originating in organ systems outside the liver. We hypothesized that direct PCB effects in the liver would induce transcriptional changes that could be mapped to pathways and processes related to $\mathrm{NASH}$.

A mouse model of diet-induced obesity and PCB coexposure has been evaluated in our laboratory, as previously reported ${ }^{23}$. Notable findings from this study included the fact that exposure to PCBs decreased fasting glucose in control mice, but had had no discernable effect on histological or serological liver damage without dietary coexposure (high-fat diet, HFD). With dietary coexposure, there was more histologically evident liver damage at a moderate exposure to PCBs (HFD-A20) than at high exposure (HFD-A200). High fat diet increased body weight and fat pad weight at all three PCB exposure levels, though it was significantly less at the high dose.

Therefore, HFD and PCB exposure contribute to the phenotype of liver damage and metabolic dysfunction in this mouse model. Moreover, because the Anniston populations were observed to have high prevalence of obesity, serologic indicators of liver injury and elevations in 
IL-6 and tPAI 1 at PCB exposures at least 10 -fold lower than the $200 \mathrm{mg} / \mathrm{kg}$ dose, the HFD+20mg/kg conditions most accurately models the PCB/dietary coexposures in Anniston. We hypothesized, therefore, that HFD coexposures would modulate the PCB-dependent transcriptome (or vice versa), producing transcriptional changes related to the phenotype in this model and in ACHS. We performed RNAseq analysis on RNA isolated from liver tissue in these animals, and performed in silico analysis of the differential transcriptome. Specifically, we expected to find that the genes differentially transcribed between $C D / v e h i c l e$ and $C D / P C B$ treatment would be enriched in gene ontology terms related to insulin resistance, and genes differentially transcribed between CD/PCB and HFD/PCB would be enriched in terms related to liver diseases, metabolic diseases, and inflammation. 


\section{CHAPTER TWO: MATERIALS AND METHODS}

Design of the animal study

In this chronic (12-week) study, male C57BI/6J mice (8 weeks old; The Jackson Laboratory, BarHarbor,ME, USA) were assigned to 6 study groups $(n=10)$ as summarized in Figure 7. Beginning at week 0 , each group received one of two diets: control diet (CD, $10.2 \%$ kCal from fat; TD.06416 Harlan Teklad) or a high fat diet (HFD, 42\% kCal from milk fat; TD.88137 Harlan Teklad), and were allowed one week to acclimate. Beginning at Week 1, each group received one of 3 exposures administered in corn oil by oral gavage: corn oil vehicle, Aroclor 1260 (AccuStandard, CT, USA) (vs. corn oil alone) at $20 \mathrm{mg} / \mathrm{kg}$ or at $200 \mathrm{mg} / \mathrm{kg}$. Importantly, the $20 \mathrm{mg} / \mathrm{kg}$ exposure was designed to mimic the highest human PCB exposures in the ACHS cohort, while the $200 \mathrm{mg} / \mathrm{kg}$ exposure was similar to that used in rodent carcinogenesis studies $^{102}$. The two doses of Aroclor 1260 were administered over a different time period in order to manage acute toxicity at the higher dose, with the $20 \mathrm{mg} / \mathrm{kg}$ dose administered once (Week 1 ) and the $200 \mathrm{mg} / \mathrm{kg}$ dose administered as four $50 \mathrm{mg} / \mathrm{kg}$ doses (Weeks 1, 3, 5, and 7).

Mice were housed in a temperature- and light-controlled room (12 h light; $12 \mathrm{~h}$ dark) with food and water ad libitum. The animals were euthanized (ketamine/xylazine, 100/20 mg/kg body weight, i.p.) at the end of Week 12. Liver tissue was harvested and immediately disrupted in RNA Stat-60 reagent (AMS Biotechnology Limited, Abingdon UK) and stored on ice.

Preparation of RNA samples and RNAseq analysis

RNA was extracted by the RNA Stat-60 protocol according to manufacturer directions. For RNAseq analysis, RNA samples were multiplexed using sequence barcoding, and sequenced single ended to 75 base pair reads using a NextSeq500 to an approximate read count of 40 


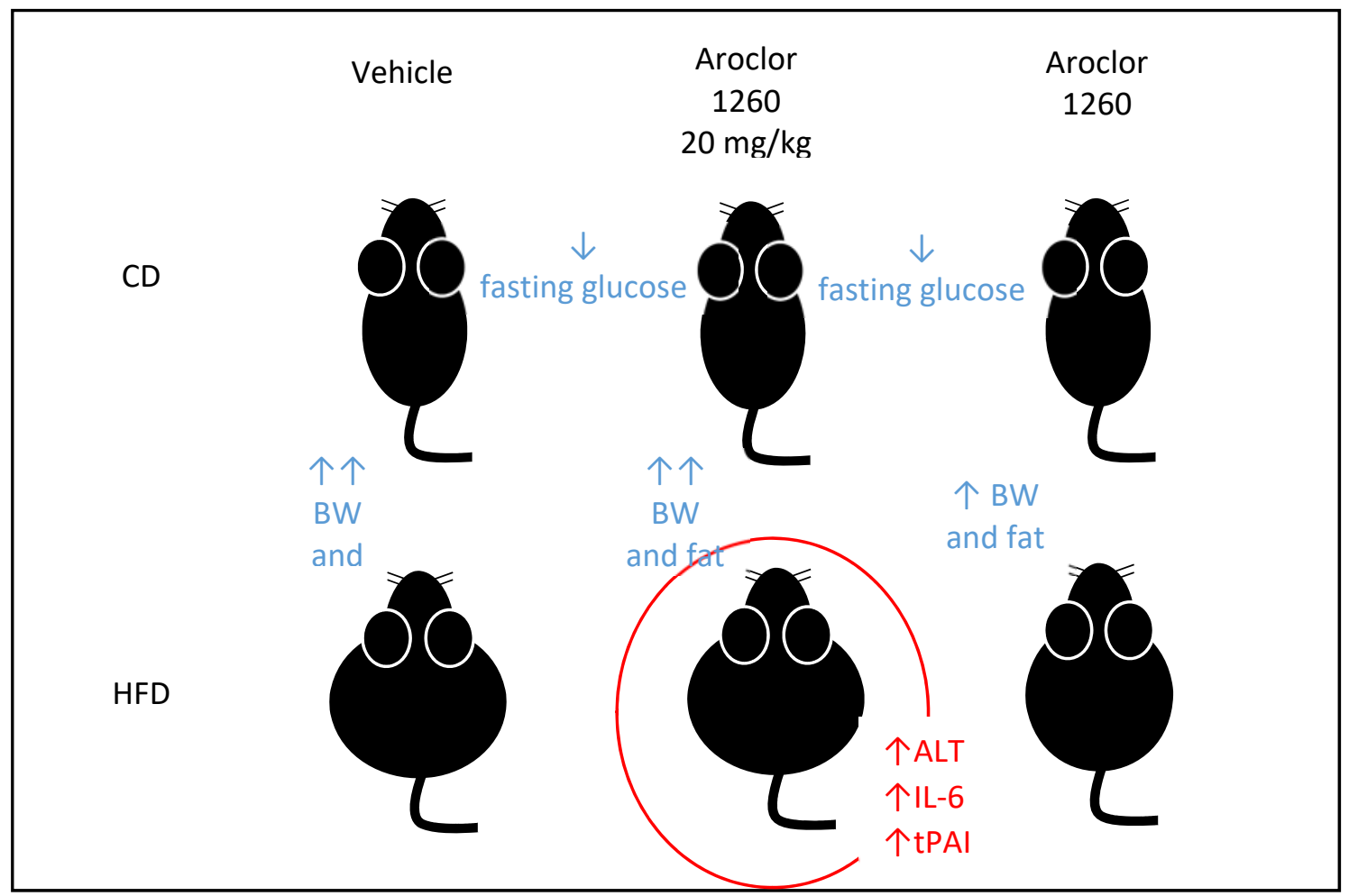

Figure 8. PCB exposure and high-fat diet induce phenotypic changes in C57/BL6 mice.

Six conditions evaluated in our mouse study are shown above, along with major phenotypic changes. 
million reads per sample. Base calling (assigning nucleobase identity to chromatogram peaks) for these files was accomplished using Illumina's bcl2fastq software. All sequences produced were aligned to the mouse reference genome $(\mathrm{GRCm} 38.83)$ using the alignment software TopHat, and transcript expression levels were calculated in FPKM (fragments per kilobase of transcript per million mapped reads) units using Cufflinks ${ }^{122}$. The quantification was guided by transcriptome annotation for the mouse downloaded from NCBI. Records corresponding to both mitochondrial and ribosomal RNA were removed (annotated as transcript_biotype rRNA or Mt_tRNA) to improve the accuracy of the transcriptome quantification. Differential analyses (FPKM units averaged from 4 replicates of each test vs. DMSO-treated controls) were performed using CuffDiff.

qPCR validation of selected targets

Targets for validation were prototypical targets of the transcription factors AhR, CAR, and PXR. From the isolated RNA samples, cDNA was generated using the QuantiTect Reverse Transcription kit (Qiagen \#205313) according to manufacturer recommendations. Multiplexed qPCR (target and GAPDH) was carried out on BioRad CFX384 system using the following TaqMan Gene Expression Array probes: Cyp1a2 (Applied Biosystems Mm00487224_m1/FAM), Cyp3a11 (Applied Biosystems Mm00731567_m1/FAM), Cyp2b10 (Applied Biosystems Mm01972453_s1/FAM) and GAPDH (Applied Biosystems, Mm99999915_g1/VIC).

Pathway and enrichment analyses

Initial analysis of the datasets were performed using the MetaCore ${ }^{\mathrm{TM}}$ (Thompson Reuters) software suite. For each treatment, the fold changes and $p$-values for each differentially transcribed gene (indexed by Ensembl ID) were uploaded into MetaCore ${ }^{\mathrm{TM}}$ and recognized genes were associated with one or more MetaCore ${ }^{\mathrm{TM}}{ }_{-}$curated network objects. Prefilters were applied to each dataset using a species (M. musculus) and tissue (liver) prefilter. Most of the raw Ensembl IDs for targets identified as differentially transcribed in each experimental comparison 
were successfully mapped to network objects represented within the MetaCore ${ }^{\mathrm{TM}}$ curated database (Table 32). An additional species and tissue prefilter applied to each dataset resulted in object sets that were approximately $70 \%$ of the original size. The number of objects which could be incorporated into one or more enrichment categories are also shown in Table 32. The fewest objects were represented within disease enrichments (2-5\% of each set), with the most represented in GO processes (approximately 90-95\%). There were no major discrepancies between groups in representation within specific MetaCore ${ }^{\mathrm{TM}}$ ontologies.

The experiments listed above were analyzed individually using the MetaCore ${ }^{\mathrm{TM}}$ Enrichment Analysis Workflow tool. Vehicle vs. PCB exposure within diet groups are represented by Groups 1 and 2, while Group 3 compares CD to HFD at 0 (Vehicle), $20 \mathrm{mg} / \mathrm{kg}$ or $200 \mathrm{mg} / \mathrm{kg}$.

The four PCB exposures vs. vehicle control experiments were analyzed using the Toxicity Workflow (Liver Toxicity filter) and Biomarkers Analysis (Metabolic Disease filter) to explore enrichments specific to liver toxicity and metabolic disease, respectively. Genomic data from these experiments was matched to maps, networks and biomarker lists from the peer-reviewed literature database manually curated by Thomson Reuters.

Because xenobiotic receptors such as PXR, CAR and AhR are transcription factors which are expected to mediate the transcriptional response to PCBs, as well as other xenobiotic and endobiotic molecules, we assessed the differential expression of MetaCore ${ }^{\mathrm{TM}}$-curated transcriptional targets of these receptors and their heterodimer complexes (PXR/RXR-alpha, CAR/RXR-alpha, and AhR/ARNT).

Using the Build Network tool within MetaCore ${ }^{\mathrm{TM}}$, we analyzed connectivity between network objects in each dataset and specific transcription factors, with the ten transcription factors which could be expected to modulate transcription of the largest number of targets within each dataset ranked highest. Because the xenobiotic receptors and heterodimer complexes are additionally expected to crosstalk with other transcription factors by protein-protein interactions, we built shortest-path networks showing the relationships between xenobiotic receptors and their binding partners which have defined relationships curated in MetaCore ${ }^{\mathrm{TM}}$. 


\section{Table 32}

in vivo experiment dataset properties

\begin{tabular}{|c|c|c|c|c|c|c|c|c|}
\hline \multirow{2}{*}{ 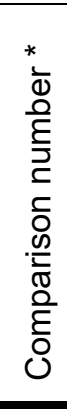 } & \multirow[b]{2}{*}{ Comparisons } & \multirow[b]{2}{*}{$\begin{array}{l}\text { \# } \\
\text { significant } \\
\text { DTGs }\end{array}$} & \multirow[b]{2}{*}{$\begin{array}{l}\text { Metacore } \\
\text { network objects } \\
\text { (unfiltered) }\end{array}$} & \multirow[b]{2}{*}{$\begin{array}{l}\text { MetaCore } \\
\text { network } \\
\text { objects } \\
\text { (prefiltered) }\end{array}$} & \multicolumn{4}{|c|}{$\begin{array}{l}\text { Included in Metacore } \\
\text { groupings }\end{array}$} \\
\hline & & & & & $\stackrel{\mathscr{0}}{\stackrel{0}{\pi}}$ & $\begin{array}{l}\mathscr{D} \\
\mathbb{D} \\
\mathbb{D} \\
\Phi \\
\Phi \\
=0\end{array}$ & $\begin{array}{l}\text { D } \\
\mathbb{N} \\
\mathbb{D} \\
\mathbb{O} \\
0 \\
0 \\
0 \\
0\end{array}$ & 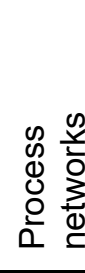 \\
\hline & \multicolumn{8}{|c|}{ Group 1 (Dose within CD) } \\
\hline 1 & CVvsC20 & 132 & 128 & 94 & 56 & 3 & 88 & 49 \\
\hline 2 & CVvsC200 & 1139 & 1088 & 825 & 327 & 17 & 736 & 306 \\
\hline & \multicolumn{8}{|c|}{ Group 2 (Dose within HFD) } \\
\hline 3 & $\mathrm{HVvsH} 20$ & 793 & 750 & 544 & 262 & 12 & 520 & 219 \\
\hline 4 & HVvsH200 & 410 & 388 & 280 & 142 & 13 & 262 & 125 \\
\hline & \multicolumn{8}{|c|}{ Group 3 (Diet within dose) } \\
\hline 5 & $\mathrm{C} 20 \mathrm{vsH} 20$ & 1436 & 1365 & 996 & 456 & 27 & 899 & 406 \\
\hline 6 & $\mathrm{C} 200 \mathrm{vsH} 200$ & 1446 & 1379 & 1007 & 487 & 30 & 936 & 419 \\
\hline 7 & CVvsHV & 1877 & 1801 & 1376 & 620 & 38 & 1256 & 564 \\
\hline
\end{tabular}

*The number associated with each comparison (column 1) is used throughout this section in the pathway maps generated with MetaCore software.

The six comparisons listed in column 2 were used to generate datasets of significantly

differentially transcribed genes (column 3). Changes to the overall sizes of the datasets were

induced by import into the MetaCore pathway analysis software and reconciliation with the

MetaCore curated database (column 4), and the application of prefilters excluding targets not

associated with species (Mus musculus) or tissue (liver) (column 5). Numbers of objects

associated each of 4 core grouping mechanisms curated by MetaCore - maps, diseases, gene

ontology (GO) processes, and process networks - are shown for each dataset in columns 6-9. 
CHAPTER TWO: RESULTS

$P C B$ exposure induced differential transcription with and without HFD coexposure

Seven comparisons were made between the aggregated RNAseq data from each condition and its respective control using CuffDiff, as indicated in Table 32. Metacore $^{\mathrm{TM}}$ enrichment analyses for the DTG sets resulting from each comparison are shown in Tables 3339.

Chronic PCB exposure in the control-diet-fed animals explored the effects of systemic PCB exposure alone on liver tissue. In the CVvsC20 comparison, DTGs were enriched in diseases related to nutritional and metabolic disease, obesity and overnutrition, although these mice were fed regular synthetic diet (Table 33). Terms related to circadian rhythm were enriched among maps, networks and processes within this DTG set as well.

The $200 \mathrm{mg} / \mathrm{kg}$ PCB exposure was a concentration based on rodent toxicity studies. Compared to vehicle, DTGs in this exposure were unsurprisingly enriched in terms related to previously reported sequelae of high-level PCB exposures: neoplastic disease biomarkers, immune-system and inflammatory process maps and networks. Disruption of cell regulatory processes was also indicated at this exposure level. Interestingly, processes and networks related to epithelial-mesenchymal transition and cell identity which are involved in both development and tissue remodeling (Wnt, TGF $\beta$ signaling), were over-represented in this dataset and, to a lesser extent, in the lower PCB exposure (Table 34).

Our evaluation of chronic moderate and high PCB exposures in the high-fat diet fed animals explored the interaction between diet and exposure, to more faithfully reconstruct conditions in many human PCB exposures, including Anniston. In the HVvsH2O and HVvsH200 comparisons, we generated datasets with genes significantly differentially transcribed in 
Table 33

Enrichments for genes differentially transcribed in comparison CVvsC20

\begin{tabular}{|c|c|c|}
\hline Diseases & p-value & FDR \\
\hline Colonic Diseases & $1.271 \mathrm{E}-11$ & 1.169E-08 \\
\hline In-house Adverse Events & $2.304 \mathrm{E}-11$ & 1.169E-08 \\
\hline Drug-Related Side Effects and Adverse Reactions & $2.949 \mathrm{E}-11$ & 1.169E-08 \\
\hline Nutritional and Metabolic Diseases & $3.875 \mathrm{E}-11$ & 1.169E-08 \\
\hline Cardiovascular Diseases & $7.116 \mathrm{E}-11$ & 1.506E-08 \\
\hline Chemically-Induced Disorders & $7.488 \mathrm{E}-11$ & 1.506E-08 \\
\hline Obesity & $1.554 \mathrm{E}-10$ & $2.280 \mathrm{E}-08$ \\
\hline Overnutrition & $1.554 \mathrm{E}-10$ & $2.280 \mathrm{E}-08$ \\
\hline Vascular Diseases & $1.712 \mathrm{E}-10$ & $2.280 \mathrm{E}-08$ \\
\hline Physiological Phenomena & $2.045 \mathrm{E}-10$ & $2.280 \mathrm{E}-08$ \\
\hline Networks & p-value & FDR \\
\hline Neurophysiological process Circadian rhythm & 2.999E-04 & 3.029E-02 \\
\hline Development Hedgehog signaling & 3.478E-03 & 1.756E-01 \\
\hline Proliferation Negative regulation of cell proliferation & 1.424E-02 & $2.721 \mathrm{E}-01$ \\
\hline $\begin{array}{l}\text { Regulation of metabolism Bile acid regulation of lipid metabolism } \\
\text { and negative FXR-dependent regulation of bile acids concentration }\end{array}$ & 1.795E-02 & 2.721E-01 \\
\hline Chemotaxis & $2.210 \mathrm{E}-02$ & $2.721 \mathrm{E}-01$ \\
\hline Cell adhesion Cell-matrix interactions & $2.427 \mathrm{E}-02$ & $2.721 \mathrm{E}-01$ \\
\hline Cytoskeleton Intermediate filaments & $2.537 \mathrm{E}-02$ & $2.721 \mathrm{E}-01$ \\
\hline Reproduction Progesterone signaling & $2.560 \mathrm{E}-02$ & $2.721 \mathrm{E}-01$ \\
\hline $\begin{array}{l}\text { Development EMT Regulation of epithelial-to-mesenchymal } \\
\text { transition }\end{array}$ & 3.091E-02 & $2.721 \mathrm{E}-01$ \\
\hline$\overline{\text { Development Neurogenesis Axonal guidance }}$ & $3.354 \mathrm{E}-02$ & $2.721 \mathrm{E}-01$ \\
\hline Maps & p-value & FDR \\
\hline Neurophysiological process Circadian rhythm & 1.656E-04 & 3.987E-02 \\
\hline Action of GSK3 beta in bipolar disorder & $3.242 \mathrm{E}-04$ & 3.987E-02 \\
\hline DNA damage ATM/ATR regulation of G1/S checkpoint & 8.741E-04 & 7.167E-02 \\
\hline Development TGF-beta receptor signaling & $3.202 \mathrm{E}-03$ & 1.199E-01 \\
\hline Development GM-CSF signaling & 3.202E-03 & 1.199E-01 \\
\hline Development WNT signaling pathway. Part 2 & $3.780 \mathrm{E}-03$ & 1.199E-01 \\
\hline Development YAP/TAZ-mediated co-regulation of transcription & 4.416E-03 & 1.199E-01 \\
\hline Nicotine metabolism in liver & 4.416E-03 & 1.199E-01 \\
\hline Immune response IL-5 signaling via JAK/STAT & $4.642 \mathrm{E}-03$ & 1.199E-01 \\
\hline Effect of H. pylori infection on gastric epithelial cell proliferation & 4.875E-03 & 1.199E-01 \\
\hline Processes & p-value & FDR \\
\hline cellular response to chemical stimulus & $4.435 \mathrm{E}-17$ & $1.807 \mathrm{E}-13$ \\
\hline circadian rhythm & $1.032 \mathrm{E}-15$ & 2.103E-12 \\
\hline rhythmic process & $1.591 \mathrm{E}-15$ & $2.161 \mathrm{E}-12$ \\
\hline cellular response to oxygen-containing compound & $6.827 \mathrm{E}-13$ & $6.955 \mathrm{E}-10$ \\
\hline regulation of developmental process & $6.009 \mathrm{E}-12$ & 3.794E-09 \\
\hline regulation of cell differentiation & $6.012 \mathrm{E}-12$ & 3.794E-09 \\
\hline response to chemical & $6.518 \mathrm{E}-12$ & 3.794E-09 \\
\hline cellular response to organic substance & $1.324 \mathrm{E}-11$ & $6.743 \mathrm{E}-09$ \\
\hline regulation of circadian rhythm & 1.493E-11 & $6.759 \mathrm{E}-09$ \\
\hline response to oxygen-containing compound & 1.939E-11 & 7.900E-09 \\
\hline
\end{tabular}


Table 34

Enrichments for genes differentially transcribed in comparison CVvsC200

\begin{tabular}{|c|c|c|}
\hline Diseases & p-value & FDR \\
\hline Neoplasms & 1.106E-19 & $1.136 \mathrm{E}-16$ \\
\hline Pathological Conditions, Signs and Symptoms & $1.280 \mathrm{E}-19$ & 1.136E-16 \\
\hline Gastrointestinal Neoplasms & 4.616E-19 & $2.488 \mathrm{E}-16$ \\
\hline Neoplasms by Site & $6.790 \mathrm{E}-19$ & $2.488 \mathrm{E}-16$ \\
\hline Respiratory Tract Neoplasms & $7.238 \mathrm{E}-19$ & $2.488 \mathrm{E}-16$ \\
\hline In-house Adverse Events & 9.070E-19 & $2.488 \mathrm{E}-16$ \\
\hline Gastrointestinal Diseases & $9.810 \mathrm{E}-19$ & $2.488 \mathrm{E}-16$ \\
\hline Drug-Related Side Effects and Adverse Reactions & $2.239 \mathrm{E}-18$ & $4.968 \mathrm{E}-16$ \\
\hline Lung Neoplasms & 4.384E-18 & $8.646 \mathrm{E}-16$ \\
\hline Chemically-Induced Disorders & $5.316 \mathrm{E}-18$ & $9.436 \mathrm{E}-16$ \\
\hline Networks & p-value & FDR \\
\hline Protein folding Response to unfolded proteins & 1.973E-08 & $3.058 \mathrm{E}-06$ \\
\hline Protein folding Folding in normal condition & $1.576 \mathrm{E}-05$ & 1.222E-03 \\
\hline Reproduction Feeding and Neurohormone signaling & 7.931E-05 & $3.470 \mathrm{E}-03$ \\
\hline Development EMT Regulation of EMT & 8.954E-05 & $3.470 \mathrm{E}-03$ \\
\hline Signal transduction WNT signaling & 1.304E-04 & $4.041 \mathrm{E}-03$ \\
\hline Inflammation Amphoterin signaling & 1.777E-04 & 4.207E-03 \\
\hline Cell cycle G1-S Growth factor regulation & 1.900E-04 & 4.207E-03 \\
\hline Apoptosis Endoplasmic reticulum stress pathway & $3.415 \mathrm{E}-04$ & $6.617 \mathrm{E}-03$ \\
\hline Signal Transduction TGF-beta, GDF and Activin signaling & 4.710E-04 & 8.112E-03 \\
\hline Inflammation IL-6 signaling & $6.253 \mathrm{E}-04$ & 9.693E-03 \\
\hline Maps & p-value & FDR \\
\hline Immune response HMGB1/RAGE signaling pathway & $1.601 \mathrm{E}-06$ & $1.242 \mathrm{E}-03$ \\
\hline Immune response IL-33 signaling pathway & 4.092E-06 & $1.564 \mathrm{E}-03$ \\
\hline Development GM-CSF signaling & $6.840 \mathrm{E}-06$ & $1.564 \mathrm{E}-03$ \\
\hline Development Gastrin in cell growth and proliferation & 8.063E-06 & 1.564E-03 \\
\hline CFTR folding and maturation (normal and CF) & 1.214E-05 & $1.884 \mathrm{E}-03$ \\
\hline Immune response IL-4 signaling pathway & $2.083 \mathrm{E}-05$ & 2.323E-03 \\
\hline Immune response TLR2 and TLR4 signaling pathways & $2.326 \mathrm{E}-05$ & 2.323E-03 \\
\hline $\begin{array}{l}\text { Development TGF-beta-dependent induction of EMT via RhoA, } \\
\text { PI3K and ILK }\end{array}$ & 2.395E-05 & 2.323E-03 \\
\hline $\begin{array}{l}\text { Development Growth factors in regulation of oligodendrocyte } \\
\text { precursor cell survival }\end{array}$ & $3.183 \mathrm{E}-05$ & 2.745E-03 \\
\hline Apoptosis and survival p53-dependent apoptosis & 4.710E-05 & $3.655 \mathrm{E}-03$ \\
\hline Processes & p-value & FDR \\
\hline negative regulation of biological process & $6.174 \mathrm{E}-24$ & $3.243 E-20$ \\
\hline regulation of response to stimulus & 1.167E-23 & $3.243 E-20$ \\
\hline regulation of apoptotic process & 1.725E-23 & $3.243 \mathrm{E}-20$ \\
\hline regulation of programmed cell death & $1.820 \mathrm{E}-23$ & $3.243 \mathrm{E}-20$ \\
\hline regulation of signal transduction & $2.106 \mathrm{E}-23$ & $3.243 E-20$ \\
\hline regulation of cell death & $6.601 \mathrm{E}-23$ & $8.472 \mathrm{E}-20$ \\
\hline negative regulation of cellular process & 8.781E-23 & 9.659E-20 \\
\hline response to organic substance & $4.861 \mathrm{E}-22$ & $4.679 \mathrm{E}-19$ \\
\hline regulation of cell communication & 1.172E-21 & $1.002 \mathrm{E}-18$ \\
\hline regulation of signaling & $3.931 \mathrm{E}-21$ & $3.027 \mathrm{E}-18$ \\
\hline
\end{tabular}


Table 35

Enrichments for genes differentially transcribed in comparison $\mathrm{HVvsH} 20$

\begin{tabular}{|c|c|c|}
\hline Diseases & p-value & FDR \\
\hline Drug-Related Side Effects and Adverse Reactions & $1.034 \mathrm{E}-34$ & 1.907E-31 \\
\hline Chemically-Induced Disorders & $4.035 \mathrm{E}-34$ & $3.721 \mathrm{E}-31$ \\
\hline In-house Adverse Events & 9.344E-33 & $5.744 \mathrm{E}-30$ \\
\hline Pathologic Processes & $4.832 \mathrm{E}-32$ & 2.227E-29 \\
\hline Pathological Conditions, Signs and Symptoms & $1.681 \mathrm{E}-31$ & $6.122 \mathrm{E}-29$ \\
\hline Ovarian Diseases & 2.159E-31 & $6.122 \mathrm{E}-29$ \\
\hline Adnexal Diseases & 2.324E-31 & $6.122 \mathrm{E}-29$ \\
\hline Gonadal Disorders & $2.441 \mathrm{E}-30$ & 5.627E-28 \\
\hline Ovarian Neoplasms & $2.986 \mathrm{E}-30$ & 6.117E-28 \\
\hline Phenomena and Processes & $5.235 \mathrm{E}-30$ & $9.653 \mathrm{E}-28$ \\
\hline Networks & p-value & FDR \\
\hline Inflammation IL-6 signaling & 7.402E-09 & 1.147E-06 \\
\hline Blood coagulation & 3.335E-08 & $2.584 \mathrm{E}-06$ \\
\hline Development Blood vessel morphogenesis & $2.242 \mathrm{E}-06$ & 1.158E-04 \\
\hline Development EMT Regulation of EMT & 1.976E-05 & 7.655E-04 \\
\hline Reproduction Gonadotropin regulation & 3.205E-05 & 9.937E-04 \\
\hline Cell adhesion Attractive and repulsive receptors & 1.921E-04 & 4.604E-03 \\
\hline Reproduction FSH-beta signaling pathway & $2.121 \mathrm{E}-04$ & 4.604E-03 \\
\hline Cell cycle G1-S Growth factor regulation & $2.376 \mathrm{E}-04$ & 4.604E-03 \\
\hline Signal transduction ESR1-nuclear pathway & 2.984E-04 & $5.138 \mathrm{E}-03$ \\
\hline Inflammation Kallikrein-kinin system & 3.730E-04 & $5.781 \mathrm{E}-03$ \\
\hline Maps & p-value & FDR \\
\hline $\begin{array}{l}\text { Immune response IL-6-induced acute-phase response in } \\
\text { hepatocytes }\end{array}$ & $5.092 \mathrm{E}-13$ & 3.753E-10 \\
\hline Reproduction Gonadotropin-releasing hormone $(\mathrm{GnRH})$ signaling & $9.800 \mathrm{E}-07$ & 2.408E-04 \\
\hline Immune response IL-6 signaling pathway via JAK/STAT & 9.800E-07 & $2.408 \mathrm{E}-04$ \\
\hline Blood coagulation Blood coagulation & 1.335E-06 & $2.460 \mathrm{E}-04$ \\
\hline Immune response IL-5 signaling via JAK/STAT & 4.937E-06 & 7.277E-04 \\
\hline Mitogenic action of Estradiol / ESR1 (nuclear) in breast cancer & 1.176E-05 & 1.444E-03 \\
\hline Signal transduction mTORC2 downstream signaling & 2.503E-05 & $2.636 \mathrm{E}-03$ \\
\hline Blood coagulation Platelet microparticle generation & 4.165E-05 & 3.567E-03 \\
\hline $\begin{array}{l}\text { Immune response MIF-induced cell adhesion, migration and } \\
\text { angiogenesis }\end{array}$ & 4.763E-05 & $3.567 \mathrm{E}-03$ \\
\hline $\begin{array}{l}\text { Development Ligand-dependent activation of the ESR1/AP-1 } \\
\text { pathway }\end{array}$ & 4.840E-05 & 3.567E-03 \\
\hline Processes & p-value & FDR \\
\hline response to organic substance & $1.741 \mathrm{E}-44$ & $1.269 \mathrm{E}-40$ \\
\hline response to chemical & $5.052 \mathrm{E}-38$ & 1.841E-34 \\
\hline cellular response to chemical stimulus & $9.611 \mathrm{E}-37$ & $2.336 \mathrm{E}-33$ \\
\hline response to oxygen-containing compound & $6.073 \mathrm{E}-33$ & 1.107E-29 \\
\hline response to stress & 9.920E-28 & 1.446E-24 \\
\hline response to organic cyclic compound & $1.411 \mathrm{E}-27$ & 1.714E-24 \\
\hline response to hormone & $3.131 \mathrm{E}-25$ & $3.261 \mathrm{E}-22$ \\
\hline positive regulation of multicellular organismal process & $6.891 \mathrm{E}-25$ & $6.279 \mathrm{E}-22$ \\
\hline response to lipid & $2.898 \mathrm{E}-24$ & $2.348 \mathrm{E}-21$ \\
\hline cellular response to organic substance & 7.733E-24 & 5.637E-21 \\
\hline \multicolumn{3}{|c|}{$\begin{array}{l}\text { Enrichment is based on the significantly differentially transcribed genes }(\mathrm{p}<.05) \text { in a } \\
\text { comparison between high-fat diet/vehicle and high-fat diet/20 mg/kg PCB }(\mathrm{HVvsH} \text { - Top ten } \\
\text { enrichments for each grouping, ranked from most to least significant by } \mathrm{p} \text {-value. }\end{array}$} \\
\hline
\end{tabular}




\section{Table 36}

Enrichments for genes differentially transcribed in comparison $\mathrm{HVvsH} 200$

\begin{tabular}{|c|c|c|}
\hline Diseases & p-value & FDR \\
\hline Pathologic Processes & $1.902 \mathrm{E}-22$ & $2.574 \mathrm{E}-19$ \\
\hline Pathological Conditions, Signs and Symptoms & $3.351 \mathrm{E}-20$ & $2.267 \mathrm{E}-17$ \\
\hline Neoplasms, Ductal, Lobular, and Medullary & $9.171 \mathrm{E}-20$ & 4.136E-17 \\
\hline Neoplasms, Glandular and Epithelial & $6.602 \mathrm{E}-19$ & $2.233 \mathrm{E}-16$ \\
\hline Neoplasms, Neuroepithelial & $3.418 \mathrm{E}-18$ & $9.250 \mathrm{E}-16$ \\
\hline In-house Adverse Events & $1.007 \mathrm{E}-17$ & $2.022 \mathrm{E}-15$ \\
\hline Digestive System Neoplasms & 1.046E-17 & $2.022 \mathrm{E}-15$ \\
\hline Drug-Related Side Effects and Adverse Reactions & 1.713E-17 & $2.639 \mathrm{E}-15$ \\
\hline Carcinoma & 1.986E-17 & 2.639E-15 \\
\hline Stomach Diseases & 1.996E-17 & $2.639 \mathrm{E}-15$ \\
\hline$\overline{\text { Networks }}$ & p-value & FDR \\
\hline Reproduction FSH-beta signaling pathway & 1.355E-04 & 1.350E-02 \\
\hline Development Regulation of angiogenesis & $2.381 \mathrm{E}-04$ & 1.350E-02 \\
\hline $\begin{array}{l}\text { Development EMT Regulation of epithelial-to-mesenchymal } \\
\text { transition }\end{array}$ & $2.736 \mathrm{E}-04$ & 1.350E-02 \\
\hline Apoptosis Anti-apoptosis mediated by external signals via NF-kB & 5.384E-04 & $1.758 \mathrm{E}-02$ \\
\hline Protein folding Response to unfolded proteins & 5.940E-04 & 1.758E-02 \\
\hline Protein folding Folding in normal condition & $8.931 \mathrm{E}-04$ & $2.203 E-02$ \\
\hline Signal transduction WNT signaling & 1.266E-03 & 2.677E-02 \\
\hline Proliferation Negative regulation of cell proliferation & $1.731 \mathrm{E}-03$ & $3.162 E-02$ \\
\hline Apoptosis Apoptotic nucleus & 1.923E-03 & $3.162 \mathrm{E}-02$ \\
\hline Proliferation Positive regulation cell proliferation & $2.434 \mathrm{E}-03$ & 3.602E-02 \\
\hline Maps & p-value & FDR \\
\hline Development Regulation of EMT & 7.035E-06 & 3.737E-03 \\
\hline Immune response IL-6-induced acute-phase response/hepatocytes & 1.913E-05 & 3.737E-03 \\
\hline $\begin{array}{l}\text { Development Lipoxin inhibitory action on PDGF, EGF and LTD4 } \\
\text { signaling }\end{array}$ & 1.913E-05 & 3.737E-03 \\
\hline CFTR folding and maturation (normal and CF) & 3.154E-05 & $4.620 \mathrm{E}-03$ \\
\hline Impaired Lipoxin A4 signaling in CF & $6.244 \mathrm{E}-05$ & 7.318E-03 \\
\hline Immune response IL-5 signaling via JAK/STAT & 2.709E-04 & $2.645 \mathrm{E}-02$ \\
\hline Immune response Oncostatin M signaling via JAK-Stat & 3.581E-04 & $2.998 \mathrm{E}-02$ \\
\hline $\begin{array}{l}\text { Development Regulation of lung epithelial progenitor cell } \\
\text { differentiation }\end{array}$ & 4.474E-04 & 3.277E-02 \\
\hline Regulation of Tissue factor signaling in cancer & $5.602 \mathrm{E}-04$ & $3.648 \mathrm{E}-02$ \\
\hline Immune response TNF-R2 signaling pathways & 6.931E-04 & 3.750E-02 \\
\hline Processes & p-value & FDR \\
\hline response to organic substance & $6.419 \mathrm{E}-23$ & $3.433 \mathrm{E}-19$ \\
\hline cellular response to chemical stimulus & 3.993E-21 & $1.068 \mathrm{E}-17$ \\
\hline response to oxygen-containing compound & 1.463E-19 & $2.608 \mathrm{E}-16$ \\
\hline regulation of signal transduction & $3.241 \mathrm{E}-19$ & 4.333E-16 \\
\hline response to hormone & $7.205 \mathrm{E}-19$ & 7.707E-16 \\
\hline negative regulation of cellular process & $2.385 \mathrm{E}-18$ & $2.105 \mathrm{E}-15$ \\
\hline negative regulation of biological process & 3.113E-18 & $2.105 E-15$ \\
\hline regulation of response to stimulus & $3.148 \mathrm{E}-18$ & $2.105 \mathrm{E}-15$ \\
\hline response to organic cyclic compound & 3.973E-18 & $2.361 \mathrm{E}-15$ \\
\hline regulation of cell communication & $5.115 \mathrm{E}-18$ & $2.735 \mathrm{E}-15$ \\
\hline \multicolumn{3}{|c|}{$\begin{array}{l}\text { Enrichment is based on the significantly differentially transcribed genes }(\mathrm{p}<.05) \text { in a } \\
\text { comparison between high-fat diet/vehicle and high-fat diet/200 mg/kg PCB }(\mathrm{HVvsH} H 200) \text {. Top } \\
\text { ten enrichments for each grouping, ranked from most to least significant by } \mathrm{p} \text {-value. }\end{array}$} \\
\hline
\end{tabular}


Table 37

Enrichments for genes differentially transcribed in comparison CVvsHV

\begin{tabular}{|c|c|c|}
\hline Diseases & $p$-value & FDR \\
\hline Pathological Conditions, Signs and Symptoms & $1.668 \mathrm{E}-40$ & $3.561 \mathrm{E}-37$ \\
\hline Pathologic Processes & 1.095E-38 & 1.169E-35 \\
\hline Nutritional and Metabolic Diseases & 2.753E-37 & 1.959E-34 \\
\hline Drug-Related Side Effects and Adverse Reactions & $4.662 \mathrm{E}-36$ & $2.489 \mathrm{E}-33$ \\
\hline In-house Adverse Events & $9.122 \mathrm{E}-36$ & 3.895E-33 \\
\hline Chemically-Induced Disorders & 1.856E-34 & $6.605 E-32$ \\
\hline Metabolic Diseases & $6.604 \mathrm{E}-32$ & $2.014 \mathrm{E}-29$ \\
\hline Respiratory Tract Neoplasms & $3.016 \mathrm{E}-29$ & 7.174E-27 \\
\hline Physiological Phenomena & 3.024E-29 & 7.174E-27 \\
\hline Phenomena and Processes & $1.560 \mathrm{E}-28$ & $3.330 \mathrm{E}-26$ \\
\hline Networks & p-value & FDR \\
\hline Development Regulation of angiogenesis & $2.143 \mathrm{E}-05$ & 3.387E-03 \\
\hline Cell adhesion Platelet-endothelium-leucocyte interactions & 4.700E-05 & 3.713E-03 \\
\hline Cell adhesion Cell-matrix interactions & 8.355E-05 & 4.400E-03 \\
\hline Chemotaxis & 1.403E-04 & $5.542 \mathrm{E}-03$ \\
\hline Cell adhesion Leucocyte chemotaxis & 4.519E-04 & 1.220E-02 \\
\hline Cell adhesion Integrin-mediated cell-matrix adhesion & $5.221 \mathrm{E}-04$ & 1.220E-02 \\
\hline Cell cycle G2-M & 5.407E-04 & $1.220 \mathrm{E}-02$ \\
\hline $\begin{array}{l}\text { Apoptosis Anti-Apoptosis mediated by external signals via } \\
\text { PI3K/AKT }\end{array}$ & $6.200 \mathrm{E}-04$ & 1.224E-02 \\
\hline Signal Transduction TGF-beta, GDF and Activin signaling & 9.318E-04 & 1.560E-02 \\
\hline Translation Translation initiation & $9.871 \mathrm{E}-04$ & 1.560E-02 \\
\hline Maps & p-value & FDR \\
\hline Transcription Sirtuin6 regulation and functions & $2.545 \mathrm{E}-09$ & $2.146 \mathrm{E}-06$ \\
\hline $\begin{array}{l}\text { Immune response IL-4-induced regulators of cell growth, survival, } \\
\text { differentiation and metabolism }\end{array}$ & 4.351E-07 & 1.834E-04 \\
\hline Cell cycle Regulation of G1/S transition (part 1) & 3.614E-06 & 1.015E-03 \\
\hline Cell cycle ESR1 regulation of G1/S transition & 7.096E-06 & 1.495E-03 \\
\hline Development TFs in segregation of hepatocytic lineage & 1.382E-05 & 2.115E-03 \\
\hline $\begin{array}{l}\text { Protein folding and maturation Angiotensin system maturation I } \\
\text { Human version }\end{array}$ & $1.505 \mathrm{E}-05$ & 2.115E-03 \\
\hline Cell adhesion ECM remodeling & 3.057E-05 & $3.018 \mathrm{E}-03$ \\
\hline Cell cycle Chromosome condensation in prometaphase & 3.389E-05 & $3.018 \mathrm{E}-03$ \\
\hline Role of ZNF202 in regulating gene expression in atherosclerosis & 3.389E-05 & $3.018 \mathrm{E}-03$ \\
\hline Cell adhesion Integrin inside-out signaling in neutrophils & $3.580 \mathrm{E}-05$ & $3.018 \mathrm{E}-03$ \\
\hline Processes & p-value & FDR \\
\hline response to organic substance & $1.024 \mathrm{E}-38$ & $9.358 \mathrm{E}-35$ \\
\hline response to oxygen-containing compound & $7.650 \mathrm{E}-31$ & $3.335 \mathrm{E}-27$ \\
\hline cellular response to chemical stimulus & $1.095 \mathrm{E}-30$ & $3.335 \mathrm{E}-27$ \\
\hline single-organism metabolic process & $8.462 \mathrm{E}-30$ & 1.933E-26 \\
\hline response to endogenous stimulus & $2.380 \mathrm{E}-28$ & 4.349E-25 \\
\hline response to organic cyclic compound & 7.309E-28 & 1.113E-24 \\
\hline response to stress & $3.846 \mathrm{E}-27$ & $5.021 \mathrm{E}-24$ \\
\hline response to external stimulus & $6.496 \mathrm{E}-27$ & 7.419E-24 \\
\hline response to lipid & $1.313 \mathrm{E}-26$ & 1.333E-23 \\
\hline response to hormone & 1.619E-26 & 1.391E-23 \\
\hline \multicolumn{3}{|c|}{$\begin{array}{l}\text { Enrichment is based on the significantly differentially transcribed genes }(p<.05) \text { in a } \\
\text { comparison between control diet/vehicle and high-fat diet/vehicle (CVvSH). Top ten } \\
\text { enrichments for each grouping, ranked from most to least significant by } p \text {-value. }\end{array}$} \\
\hline
\end{tabular}


Table 38

Enrichments for genes differentially transcribed in comparison $\mathrm{C} 20 \mathrm{vsH} 2 \mathrm{O}$

\begin{tabular}{|c|c|c|}
\hline Diseases & p-value & FDR \\
\hline Drug-Related Side Effects and Adverse Reactions & $9.926 \mathrm{E}-38$ & $2.026 \mathrm{E}-34$ \\
\hline Chemically-Induced Disorders & 4.539E-37 & $4.632 E-34$ \\
\hline In-house Adverse Events & 8.728E-37 & $5.938 E-34$ \\
\hline Pathological Conditions, Signs and Symptoms & $2.331 \mathrm{E}-36$ & 1.189E-33 \\
\hline Pathologic Processes & 3.434E-36 & 1.402E-33 \\
\hline Fibrosis & $1.512 \mathrm{E}-34$ & $5.142 \mathrm{E}-32$ \\
\hline Phenomena and Processes & 6.761E-34 & 1.971E-31 \\
\hline Physiological Phenomena & $2.105 \mathrm{E}-33$ & $5.371 \mathrm{E}-31$ \\
\hline Body Weight & $4.840 \mathrm{E}-32$ & 1.098E-29 \\
\hline Nutritional and Metabolic Diseases & 7.909E-32 & 1.614E-29 \\
\hline Networks & p-value & FDR \\
\hline Development Regulation of angiogenesis & 1.829E-09 & 2.889E-07 \\
\hline Development Blood vessel morphogenesis & 8.673E-06 & 6.851E-04 \\
\hline $\begin{array}{l}\text { Development EMT Regulation of epithelial-to-mesenchymal } \\
\text { transition }\end{array}$ & 1.716E-05 & 9.036E-04 \\
\hline Inflammation IL-6 signaling & 1.992E-04 & 6.405E-03 \\
\hline Signal Transduction BMP and GDF signaling & 2.027E-04 & $6.405 \mathrm{E}-03$ \\
\hline Inflammation Amphoterin signaling & $5.171 \mathrm{E}-04$ & 1.191E-02 \\
\hline $\begin{array}{l}\text { Regulation of metabolism Bile acid regulation of lipid metabolism } \\
\text { and negative FXR-dependent regulation of bile acids concentration }\end{array}$ & $5.276 \mathrm{E}-04$ & 1.191E-02 \\
\hline Apoptosis Endoplasmic reticulum stress pathway & 1.562E-03 & $3.086 \mathrm{E}-02$ \\
\hline Cell adhesion Attractive and repulsive receptors & 1.912E-03 & 3.357E-02 \\
\hline Blood coagulation & $2.654 \mathrm{E}-03$ & 3.839E-02 \\
\hline$\overline{\text { Maps }}$ & p-value & FDR \\
\hline Transcription Sirtuin6 regulation and functions & 8.710E-09 & 7.003E-06 \\
\hline Immune response IL-6-induced acute-phase response/hepatocytes & 8.699E-08 & 3.497E-05 \\
\hline $\begin{array}{l}\text { Development } \beta \text {-adrenergic receptors/brown adipocyte } \\
\text { differentiation }\end{array}$ & $1.118 \mathrm{E}-06$ & 2.997E-04 \\
\hline Development TGF-beta-dependent induction of EMT via MAPK & 2.196E-06 & 4.414E-04 \\
\hline Role of ZNF202 in regulating gene expression in atherosclerosis & 4.150E-06 & $6.673 \mathrm{E}-04$ \\
\hline Protein folding/maturation Angiotensin system maturation $\backslash$ Human & $5.728 \mathrm{E}-06$ & 7.675E-04 \\
\hline Protein folding/maturation Angiotensin system maturation $\backslash$ Rodent & 1.793E-05 & $2.060 \mathrm{E}-03$ \\
\hline Signal transduction mTORC1 downstream signaling & $3.867 \mathrm{E}-05$ & $3.526 \mathrm{E}-03$ \\
\hline Immune response IL-6 signaling pathway via JAK/STAT & $4.800 \mathrm{E}-05$ & $3.526 \mathrm{E}-03$ \\
\hline Apoptosis and survival Role of PKR in stress-induced apoptosis & 4.824E-05 & 3.526E-03 \\
\hline Processes & p-value & FDR \\
\hline response to oxygen-containing compound & $1.007 \mathrm{E}-43$ & $8.605 E-40$ \\
\hline response to organic substance & $5.608 \mathrm{E}-42$ & 2.397E-38 \\
\hline cellular response to chemical stimulus & $1.522 \mathrm{E}-37$ & 4.337E-34 \\
\hline response to organic cyclic compound & 4.105E-34 & $8.772 \mathrm{E}-31$ \\
\hline response to organonitrogen compound & 2.307E-31 & $3.944 \mathrm{E}-28$ \\
\hline response to chemical & 2.647E-30 & 3.770E-27 \\
\hline response to external stimulus & $3.106 \mathrm{E}-30$ & $3.792 \mathrm{E}-27$ \\
\hline response to nitrogen compound & 4.595E-30 & 4.909E-27 \\
\hline single-organism metabolic process & 1.205E-29 & 1.144E-26 \\
\hline response to lipid & 2.262E-29 & 1.933E-26 \\
\hline \multicolumn{3}{|c|}{$\begin{array}{l}\text { Enrichment is based on the significantly differentially transcribed genes }(\mathrm{p}<.05) \text { in a } \\
\text { comparison between control diet/20 mg/kg PCB and high-fat diet/20 mg/kg PCB }(\mathrm{C} 20 \mathrm{vsH} 20) \text {. } \\
\text { Top ten enrichments for each grouping, ranked from most to least significant by } \mathrm{p} \text {-value. }\end{array}$} \\
\hline
\end{tabular}


Table 39

Enrichments for genes differentially transcribed in comparison $\mathrm{C} 200 \mathrm{vsH} 200$

\begin{tabular}{|c|c|c|}
\hline Diseases & p-value & FDR \\
\hline Pathologic Processes & $2.843 \mathrm{E}-45$ & $5.814 \mathrm{E}-42$ \\
\hline Drug-Related Side Effects and Adverse Reactions & $4.265 \mathrm{E}-40$ & 4.139E-37 \\
\hline Pathological Conditions, Signs and Symptoms & $6.071 \mathrm{E}-40$ & $4.139 \mathrm{E}-37$ \\
\hline In-house Adverse Events & 4.134E-39 & $1.816 \mathrm{E}-36$ \\
\hline Chemically-Induced Disorders & $4.439 \mathrm{E}-39$ & 1.816E-36 \\
\hline Nutritional and Metabolic Diseases & $1.527 \mathrm{E}-31$ & $5.204 \mathrm{E}-29$ \\
\hline Metabolic Diseases & $8.142 E-31$ & $2.379 \mathrm{E}-28$ \\
\hline Vascular Diseases & $2.710 \mathrm{E}-29$ & $6.928 \mathrm{E}-27$ \\
\hline Endocrine System Diseases & $2.060 \mathrm{E}-28$ & $4.681 \mathrm{E}-26$ \\
\hline Cardiovascular Diseases & $7.414 \mathrm{E}-28$ & 1.516E-25 \\
\hline$\overline{\text { Networks }}$ & p-value & FDR \\
\hline Development Regulation of angiogenesis & $5.068 \mathrm{E}-06$ & 4.739E-04 \\
\hline Chemotaxis & $6.115 \mathrm{E}-06$ & 4.739E-04 \\
\hline Immune response Phagocytosis & 1.356E-05 & $7.005 \mathrm{E}-04$ \\
\hline Inflammation IL-6 signaling & 6.717E-05 & $2.603 \mathrm{E}-03$ \\
\hline Cell adhesion Attractive and repulsive receptors & 1.385E-04 & $3.664 \mathrm{E}-03$ \\
\hline Development Blood vessel morphogenesis & $1.418 \mathrm{E}-04$ & 3.664E-03 \\
\hline Cell adhesion Leucocyte chemotaxis & $2.759 \mathrm{E}-04$ & $6.108 \mathrm{E}-03$ \\
\hline Signal Transduction Cholecystokinin signaling & 1.198E-03 & $2.320 \mathrm{E}-02$ \\
\hline Cell cycle G1-S Interleukin regulation & 1.407E-03 & $2.423 \mathrm{E}-02$ \\
\hline Immune response Phagosome in antigen presentation & $1.960 \mathrm{E}-03$ & 3.037E-02 \\
\hline Maps & p-value & FDR \\
\hline $\begin{array}{l}\text { Immune response IL-4-induced regulators of cell growth, survival, } \\
\text { differentiation and metabolism }\end{array}$ & 3.816E-07 & 3.114E-04 \\
\hline Immune response IL-6-induced acute-phase response/hepatocytes & 9.473E-07 & 3.865E-04 \\
\hline Immune response IL-2 activation and signaling pathway & $3.245 \mathrm{E}-06$ & $6.912 \mathrm{E}-04$ \\
\hline Development Role of IL-8 in angiogenesis & 3.388E-06 & $6.912 \mathrm{E}-04$ \\
\hline Neurophysiological Receptor-mediated axon growth repulsion & 1.057E-05 & 1.539E-03 \\
\hline Immune response Antigen presentation by $\mathrm{MHC}$ class II & 1.161E-05 & 1.539E-03 \\
\hline Transcription Role of AP-1 in regulation of cellular metabolism & 1.320E-05 & 1.539E-03 \\
\hline Putative pathways for BPA-stimulated fat cell differentiation & 1.999E-05 & $2.039 \mathrm{E}-03$ \\
\hline Cell cycle ESR1 regulation of G1/S transition & 3.414E-05 & 3.095E-03 \\
\hline Signal transduction mTORC1 downstream signaling & 4.447E-05 & 3.629E-03 \\
\hline Processes & p-value & FDR \\
\hline response to organic substance & 1.995E-37 & $1.674 \mathrm{E}-33$ \\
\hline single-organism metabolic process & $1.987 \mathrm{E}-32$ & $8.334 \mathrm{E}-29$ \\
\hline small molecule metabolic process & $2.501 \mathrm{E}-31$ & $6.994 \mathrm{E}-28$ \\
\hline response to stress & $1.066 \mathrm{E}-29$ & 1.913E-26 \\
\hline lipid metabolic process & 1.140E-29 & 1.913E-26 \\
\hline response to oxygen-containing compound & $5.001 \mathrm{E}-29$ & $6.993 \mathrm{E}-26$ \\
\hline cellular response to chemical stimulus & $8.298 \mathrm{E}-29$ & $9.946 \mathrm{E}-26$ \\
\hline response to organic cyclic compound & $2.895 \mathrm{E}-27$ & 3.036E-24 \\
\hline response to chemical & $8.844 \mathrm{E}-27$ & $8.245 E-24$ \\
\hline response to hormone & $1.217 \mathrm{E}-26$ & $1.021 \mathrm{E}-23$ \\
\hline
\end{tabular}


HFD/PCB coexposure vs. HFD alone. In the HVvsH20 comparison, enriched diseases were associated with gonadal and ovarian disease, including ovarian neoplasms. Enriched networks and maps including both inflammatory and EMT-related categories (Table 35). The HVvsH200 comparison was notable for enrichments in inflammatory, immune-system and EMT-related networks and maps, and neoplastic diseases (Table 36). Interestingly, both of the HFD+PCB groups were highly enriched in multiple processes related to xenobiotic responses (Tables 3536), while the $C D+P C B$ groups were enriched in categories related to regulation of physiological processes (Tables 33-34).

Transcriptional effects of HFD exposure were modulated by coexposure to PCBs

Comparisons between CD and HFD at each level of PCB exposure (CVvsHV, C20vsH20, C200vsH200 comparisons) explored the transcriptional effects of HFD exposure without or with two levels of PCB coexposure (Tables 37-39). Unsurprisingly, nutritional and metabolic disease biomarkers were over-represented in all three comparisons. Enriched processes for comparisons at all levels were associated with changes in cellular response to endobiotic and xenobiotic organic cyclic compounds, with responses to endogenous compounds (e.g., hormone, lipid, etc.) featuring prominently in the CVvsHV comparison (Table 37), and responses to organonitrogens and external stimulus encroaching in the moderate exposure (Table 38). In a comparison between CD and HFD in the highest PCB exposure group, enrichments in process maps related to pro-inflammatory and anti-inflammatory cytokine signaling were over-represented (Table 39).

The sets of genes differentially transcribed by moderate and high PCB exposures within each diet partially overlapped

Figure 9 shows the relative size of the targets differentially transcribed in a comparison between vehicle and moderate (gold) or vehicle and high (red) PCB exposure in animals on control diet (Panel A) or HFD (Panel B). The overlapping areas indicate targets which are shared between moderate and high PCB exposure levels within a single diet condition. On control diet, a 


\section{Panel A - Control Diet}

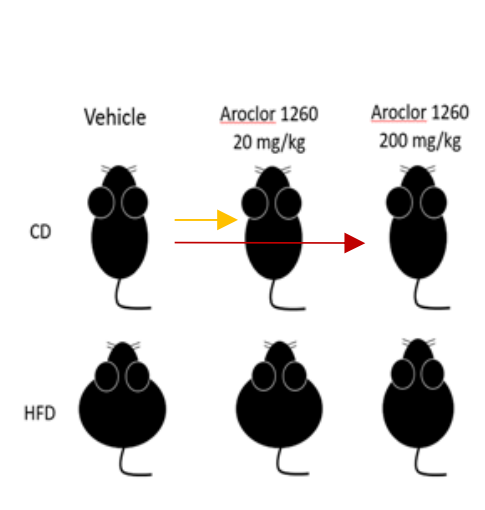

$20 \mathrm{mg} / \mathrm{kg}$

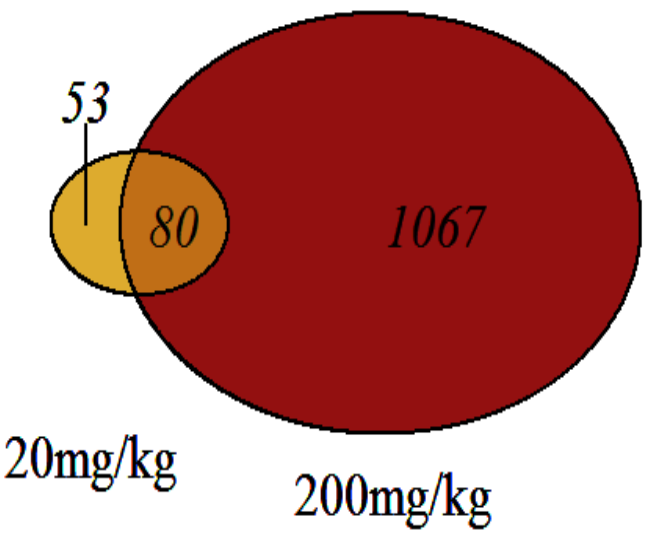

\section{Panel B - High-Fat Diet}
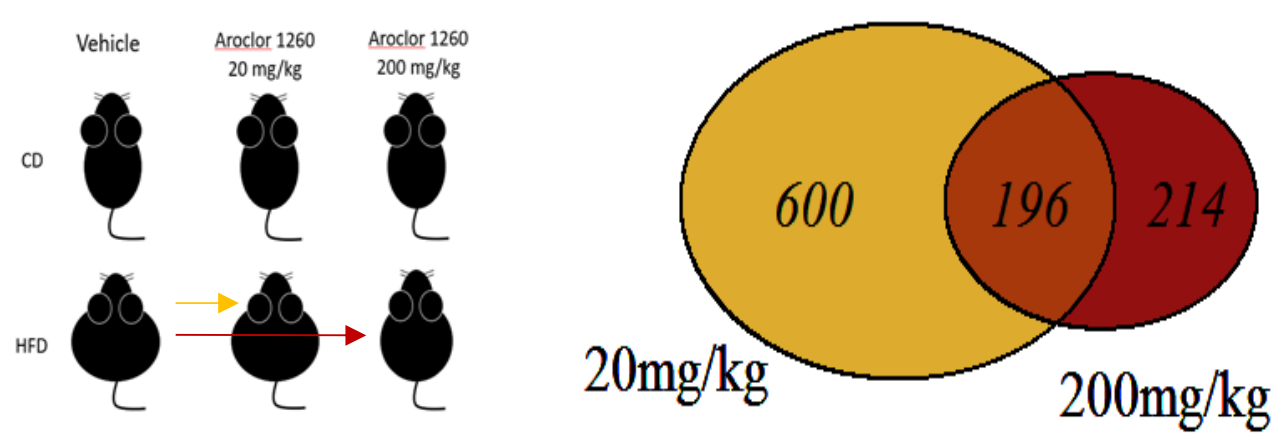

Figure 9. Moderate and high PCB exposures produce overlapping DTG sets (vs. vehicle) In an evaluation of PCB transcriptional effect, comparison between PCB exposure and control (vehicle) produced sets of significantly differentially transcribed genes (DTGs), which are shown for control diet (CD, Panel A) and high fat diet (HFD, Panel B). The number of targets which were differentially affected at both $20 \mathrm{mg} / \mathrm{kg}$ Aroclor 1260 and $200 \mathrm{mg} / \mathrm{kg}$ Aroclor 1260 are shown in the intersecting regions. Targets which were differentially regulated at only moderate (gold) or high (red) dose are shown in the non-intersecting regions. For CD, high PCB exposure resulted in a larger DTG set than moderate PCB exposure. For HFD, moderate PCB exposure resulted in a larger DTG set than high PCB exposure. 
larger number of targets were significantly differentially transcribed in response to chronic high PCB exposure (CVvsC200 comparison) than chronic moderate PCB exposure (CVvsC20 comparison). In the HFD groups, this pattern was reversed, with a smaller number of genes (both absolute and relative) differentially transcribed with the high PCB exposure (HVvsH200 comparison) and a larger number in the moderate PCB exposure (HVvsH20 comparison).

Enrichment analysis of DTGs common between moderate and high PCB exposure within the CD group are shown in Table 40. Targets perturbed in both exposures were overrepresented in circadian rhythm-related categories within networks and processes, while processes involving response to endogenous and exogenous molecules comprised the remainder of the most ten enriched processes. Interestingly, hedgehog signaling and WNT signaling, both involved in development and remodeling, were differentially regulated in both moderate and high PCB exposures within this diet group. In the HFD-fed groups, enrichment analysis of DTGs common between both moderate and high PCB exposures were enriched in several networks and maps involved in development and EMT, particularly WNT signaling (Table 41). Hormone signaling pathways including FSH, progesterone and gonadotropin were also identified as commonly engaged networks.

HFD coexposure at different PCB doses resulted in DTG sets with large areas of overlap

When high fat diet was compared to control diet within each PCB exposure level (CDvsHFD, C20vsH20, C200vsH200), three large sets of differentially transcribed genes were identified, with substantial overlap (Figure 10). In the vehicle-treated comparison (black) the entire DTG set can be described as diet-dependent, and the overlapping areas between this and other sets can be described as targets which are differentially transcribed with HFD exposure in the presence or absence of Aroclor 1260 coexposure. The top enrichments for the DTGs produced are shown in Tables 42-46. The moderate (gold) and high (red) PCB coexposuredependent DTGs are represented by the sections of those sets that do not overlap with the vehicle-treated comparison. 


\section{Table 40}

Common targets between moderate/high PCB exposure levels within the control diet groups

\begin{tabular}{|c|c|c|}
\hline Diseases & $p$-value & FDR \\
\hline In-house Adverse Events & 3.271E-07 & $1.434 \mathrm{E}-12$ \\
\hline Colonic Diseases & $3.480 \mathrm{E}-07$ & 9.982E-08 \\
\hline Drug-Related Side Effects and Adverse Reactions & 3.774E-07 & $2.375 \mathrm{E}-12$ \\
\hline Endocrine System Diseases & 9.532E-07 & 2.795E-05 \\
\hline$\overline{\text { Chemically-Induced Disorders }}$ & 1.422E-06 & $2.626 \mathrm{E}-12$ \\
\hline Cardiovascular Diseases & 1.731E-06 & $1.550 \mathrm{E}-08$ \\
\hline Neoplasms, Bone Tissue & $3.722 \mathrm{E}-06$ & $2.101 \mathrm{E}-05$ \\
\hline$\overline{\text { Vascular Diseases }}$ & $4.292 \mathrm{E}-06$ & 1.097E-08 \\
\hline Diabetes Mellitus, Type 2 & 4.349E-06 & 4.745E-04 \\
\hline Eye Diseases & $6.021 \mathrm{E}-06$ & 5.913E-04 \\
\hline Networks & p-value & FDR \\
\hline Reproduction Progesterone signaling & $2.405 \mathrm{E}-03$ & 1.465E-01 \\
\hline $\begin{array}{l}\text { Regulation of metabolism Bile acid regulation of lipid metabolism } \\
\text { and negative FXR-dependent regulation of bile acids concentration }\end{array}$ & 3.806E-03 & 1.465E-01 \\
\hline Signal transduction WNT signaling & 7.644E-03 & 2.304E-02 \\
\hline Immune response IL-5 signalling & $1.238 \mathrm{E}-02$ & $1.980 \mathrm{E}-01$ \\
\hline Reproduction Feeding and Neurohormone signaling & 1.374E-02 & 1.489E-02 \\
\hline Development Hedgehog signaling & $2.575 \mathrm{E}-02$ & 3.305E-01 \\
\hline Development Ossification and bone remodeling & $3.250 \mathrm{E}-02$ & 3.387E-01 \\
\hline Cell adhesion Glycoconjugates & 3.519E-02 & 3.387E-01 \\
\hline Muscle contraction & $4.152 \mathrm{E}-02$ & 7.278E-02 \\
\hline Neurophysiological process Circadian rhythm & 4.519E-02 & $7.038 \mathrm{E}-02$ \\
\hline Maps & p-value & FDR \\
\hline Development WNT signaling pathway. Part 2 & $6.710 \mathrm{E}-04$ & 4.347E-02 \\
\hline Nicotine metabolism in liver & 7.887E-04 & 4.347E-02 \\
\hline Immune response IL-5 signaling via JAK/STAT & 8.307E-04 & 4.347E-02 \\
\hline Action of GSK3 beta in bipolar disorder & $2.524 \mathrm{E}-03$ & $8.628 \mathrm{E}-02$ \\
\hline Immune response IL-27 signaling pathway & $2.748 \mathrm{E}-03$ & $8.628 \mathrm{E}-02$ \\
\hline $\begin{array}{l}\text { Regulation of lipid metabolism FXR-dependent negative-feedback } \\
\text { regulation of bile acids concentration }\end{array}$ & 4.564E-03 & 1.090E-01 \\
\hline DNA damage ATM/ATR regulation of G1/S checkpoint & 4.858E-03 & $9.421 \mathrm{E}-02$ \\
\hline Development EPO-induced Jak-STAT pathway & $6.121 \mathrm{E}-03$ & $1.201 \mathrm{E}-01$ \\
\hline $\begin{array}{l}\text { Regulation of metabolism Bile acids FXR regulation/ glucose and } \\
\text { lipid metabolism }\end{array}$ & 7.886E-03 & 1.376E-01 \\
\hline Regulation of GSK3 beta in bipolar disorder & 9.852E-03 & 8.940E-02 \\
\hline Processes & p-value & FDR \\
\hline cellular response to chemical stimulus & $1.811 \mathrm{E}-12$ & $6.576 \mathrm{E}-12$ \\
\hline cellular response to organic substance & 1.056E-09 & 1.657E-09 \\
\hline response to stimulus & $5.204 \mathrm{E}-09$ & $1.921 \mathrm{E}-06$ \\
\hline cellular response to oxygen-containing compound & 5.723E-09 & 4.689E-06 \\
\hline circadian rhythm & 1.362E-08 & $1.522 \mathrm{E}-06$ \\
\hline response to chemical & 2.079E-08 & 8.674E-07 \\
\hline response to organic substance & $5.529 \mathrm{E}-08$ & $2.305 \mathrm{E}-14$ \\
\hline rhythmic process & 9.143E-08 & 4.206E-07 \\
\hline response to organic cyclic compound & $1.641 \mathrm{E}-07$ & $2.153 \mathrm{E}-10$ \\
\hline cellular response to organic cyclic compound & $2.955 \mathrm{E}-07$ & $8.475 \mathrm{E}-05$ \\
\hline
\end{tabular}


Table 41

Common targets between moderate/high PCB exposure levels within the high-fat diet groups

\begin{tabular}{|c|c|c|}
\hline Diseases & $p$-value & FDR \\
\hline Hypoxia & 1.987E-16 & $2.285 \mathrm{E}-13$ \\
\hline Neoplasms, Ductal, Lobular, and Medullary & $4.844 \mathrm{E}-16$ & $2.785 \mathrm{E}-13$ \\
\hline Physiological Phenomena & $1.831 \mathrm{E}-15$ & $7.121 \mathrm{E}-15$ \\
\hline Ovarian Neoplasms & $1.840 \mathrm{E}-15$ & $2.478 \mathrm{E}-15$ \\
\hline$\overline{O \text { Ovarian Diseases }}$ & $2.609 \mathrm{E}-15$ & $3.432 \mathrm{E}-16$ \\
\hline Adnexal Diseases & $2.675 \mathrm{E}-15$ & $3.432 \mathrm{E}-16$ \\
\hline In-house Adverse Events & $2.932 \mathrm{E}-15$ & $2.308 \mathrm{E}-17$ \\
\hline Vascular Diseases & $3.321 \mathrm{E}-15$ & 5.733E-15 \\
\hline Drug-Related Side Effects and Adverse Reactions & $4.143 \mathrm{E}-15$ & $7.903 \mathrm{E}-19$ \\
\hline Signs and Symptoms, Respiratory & $4.344 \mathrm{E}-15$ & 4.996E-13 \\
\hline$\overline{\text { Networks }}$ & p-value & FDR \\
\hline Reproduction FSH-beta signaling pathway & 2.903E-05 & 3.056E-03 \\
\hline Reproduction Progesterone signaling & 5.023E-05 & 3.056E-03 \\
\hline Proliferation Positive regulation cell proliferation & $6.596 \mathrm{E}-05$ & 3.056E-03 \\
\hline Inflammation IL-6 signaling & 1.805E-04 & 9.444E-04 \\
\hline Signal transduction ESR1-membrane pathway & 2.894E-04 & $6.880 \mathrm{E}-03$ \\
\hline Development Regulation of angiogenesis & 3.587E-04 & $6.880 \mathrm{E}-03$ \\
\hline Signal transduction WNT signaling & 3.775E-04 & $6.880 \mathrm{E}-03$ \\
\hline Development EMT Regulation of EMT & 3.960E-04 & $6.880 \mathrm{E}-03$ \\
\hline Cell cycle G1-S Growth factor regulation & $7.198 \mathrm{E}-04$ & 1.112E-02 \\
\hline Reproduction Gonadotropin regulation & 8.224E-04 & 1.143E-02 \\
\hline Maps & p-value & FDR \\
\hline IL-6-induced acute-phase response/hepatocytes & 7.330E-06 & 3.084E-05 \\
\hline Lipoxin inhibitory action-PDGF/EGF/LTD4 signaling & 7.330E-06 & 1.646E-03 \\
\hline Impaired Lipoxin A4 signaling in CF & 2.013E-05 & 3.013E-03 \\
\hline Development WNT signaling pathway. Part 2 & $5.040 \mathrm{E}-05$ & $5.658 \mathrm{E}-03$ \\
\hline Effect of H. pylori infection on gastric epithelial cell proliferation & 7.811E-05 & 7.015E-03 \\
\hline Development Gastrin in cell growth and proliferation & 1.077E-04 & 7.580E-03 \\
\hline$\overline{\text { Development Regulation of EMT }}$ & $1.254 \mathrm{E}-04$ & 7.580E-03 \\
\hline Ovarian cancer (main signaling cascades) & $1.351 \mathrm{E}-04$ & 7.580E-03 \\
\hline Development Beta-adrenergic receptors transactivation of EGFR & 1.753E-04 & 8.333E-03 \\
\hline Development Ligand-dependent activation/ESR1/AP-1 pathway & 1.856E-04 & 8.333E-03 \\
\hline Processes & p-value & FDR \\
\hline response to organic substance & $2.006 \mathrm{E}-20$ & $5.752 \mathrm{E}-23$ \\
\hline cellular response to chemical stimulus & $2.280 \mathrm{E}-20$ & 4.951E-17 \\
\hline regulation of signal transduction & $4.488 \mathrm{E}-19$ & $6.498 \mathrm{E}-16$ \\
\hline regulation of cell communication & $1.713 \mathrm{E}-18$ & $1.860 \mathrm{E}-15$ \\
\hline regulation of signaling & $3.782 \mathrm{E}-18$ & $3.286 \mathrm{E}-15$ \\
\hline regulation of response to stimulus & $1.437 \mathrm{E}-17$ & $1.041 \mathrm{E}-14$ \\
\hline response to chemical & $2.895 \mathrm{E}-17$ & 8.033E-20 \\
\hline response to oxygen-containing compound & $3.049 \mathrm{E}-17$ & $1.468 \mathrm{E}-15$ \\
\hline positive regulation of cell communication & $2.363 \mathrm{E}-16$ & 1.140E-13 \\
\hline positive regulation of signaling & $2.696 \mathrm{E}-16$ & 1.171E-13 \\
\hline
\end{tabular}




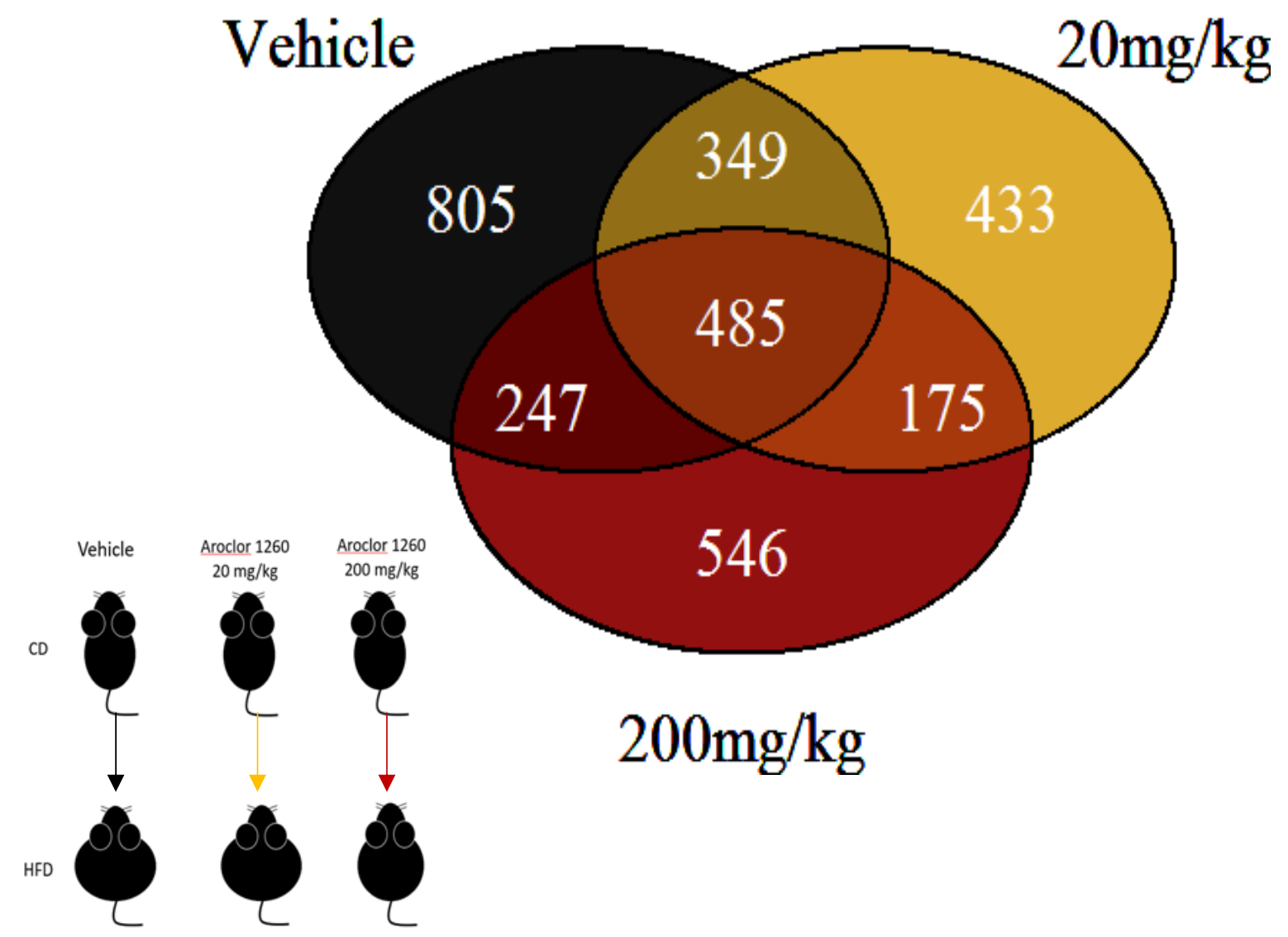

Figure 10. Comparison of PCB/Diet interactions.

For each PCB exposure, control and high fat diet were compared to generate a differentially regulated target set. These differentially regulated target sets are compared in the figure above. 
Enrichment analysis of common targets for all PCB exposure levels within diet comparisons (Table 42) showed nutritional, metabolic, and endocrine-related diseases among the highly-enriched disease biomarkers, and IL-6 signaling among the engaged maps and processes. Processes related to lipid and steroid metabolism, endobiotic and xenobiotic response were also commonly engaged in these comparisons.

Differentially regulated targets within vehicle vs. PCB comparisons were enriched in liver and metabolic disease biomarkers

Because the animal model phenotype suggested liver and metabolic disease processes at work, we further explored liver toxicity analysis and biomarker analysis for nutritional and metabolic disease within these datasets. In an evaluation of liver toxicity endpoints in the control diet groups, there were no significantly enriched pathways for the moderate exposure group (Table 43), however, the high exposure was significantly (FDR $<0.05)$ enriched in pathways related to ischemia and hypoxia (Table 44). In the high fat diet groups, significantly enriched categories within the liver toxicity analysis were cholestasis and steatosis pathways for moderate exposure (Table 45) and steatosis, fibrosis, and liver hypertrophy in the high exposure group (Table 46). Targets affected within the steatosis category are shown in Table 47, and included LIPE, ELOVL6, FADS3, GPAM, and several cytochrome P450s including members of the Cyp4A and Cyp2C subfamilies.

One liver injury pathway map which was highly enriched in DTGs from several of the conditions was IL-6-mediated acute-phase response in hepatocytes (Figure 11). Several immediate effectors of acute phase response down-stream of IL-6 signaling were up-regulated, particularly in the $\mathrm{HVvsH} 20$ condition, including serum amyloid proteins (A1, A2, and $\mathrm{A} 3$ isoforms), serum amyloid P-component, and fibrinogen components (alpha, beta, and gamma), shown in Figure 12. Engagement of this pathway of local hepatic inflammatory response is consistent with histological findings in this animal model, as well as serological findings of increased IL-6 and liver injury in both the animal model, and in the PCB-exposed population of Anniston, AL. 
Table 42

Common targets between all PCB exposure levels within diet comparisons

\begin{tabular}{|c|c|c|}
\hline Diseases & p-value & FDR \\
\hline Pathological Conditions, Signs and Symptoms & $6.116 \mathrm{E}-27$ & $8.706 \mathrm{E}-24$ \\
\hline Pathologic Processes & 1.071E-26 & 8.706E-24 \\
\hline Drug-Related Side Effects and Adverse Reactions & $2.676 \mathrm{E}-24$ & $1.450 \mathrm{E}-21$ \\
\hline In-house Adverse Events & $1.478 \mathrm{E}-23$ & $4.980 \mathrm{E}-21$ \\
\hline Chemically-Induced Disorders & $1.531 \mathrm{E}-23$ & $4.980 \mathrm{E}-21$ \\
\hline Nutritional and Metabolic Diseases & $2.480 \mathrm{E}-23$ & $6.722 \mathrm{E}-21$ \\
\hline Metabolic Diseases & 1.164E-22 & $2.704 \mathrm{E}-20$ \\
\hline$\overline{\text { Phenomena and Processes }}$ & $5.832 \mathrm{E}-22$ & 1.185E-19 \\
\hline Physiological Phenomena & 1.705E-21 & $3.081 \mathrm{E}-19$ \\
\hline Endocrine System Diseases & $4.360 \mathrm{E}-21$ & 7.089E-19 \\
\hline Networks & p-value & FDR \\
\hline Development Regulation of angiogenesis & $3.424 \mathrm{E}-07$ & $5.000 \mathrm{E}-05$ \\
\hline Inflammation IL-6 signaling & 5.307E-06 & 3.874E-04 \\
\hline Development Blood vessel morphogenesis & $3.416 \mathrm{E}-05$ & $1.662 \mathrm{E}-03$ \\
\hline Translation Translation initiation & 2.444E-04 & 8.920E-03 \\
\hline Translation Elongation-Termination & 1.509E-03 & $4.405 \mathrm{E}-02$ \\
\hline Signal Transduction Cholecystokinin signaling & $4.062 \mathrm{E}-03$ & $8.473 \mathrm{E}-02$ \\
\hline Cell cycle Meiosis & $4.062 \mathrm{E}-03$ & $8.473 \mathrm{E}-02$ \\
\hline Reproduction Feeding and Neurohormone signaling & $4.882 \mathrm{E}-03$ & $8.910 \mathrm{E}-02$ \\
\hline Cell cycle G0-G1 & $7.245 \mathrm{E}-03$ & $1.109 \mathrm{E}-01$ \\
\hline Blood coagulation & 7.594E-03 & 1.109E-01 \\
\hline Maps & p-value & FDR \\
\hline Cholesterol Biosynthesis & 7.453E-06 & $4.636 \mathrm{E}-03$ \\
\hline Cell cycle Role of Nek in cell cycle regulation & 3.880E-05 & 1.207E-02 \\
\hline IL-6-induced acute-phase response/hepatocytes & 7.792E-05 & 1.616E-02 \\
\hline$\overline{\text { Effect of H. pylori infection on gastric epithelial cell proliferation }}$ & 1.643E-04 & $2.555 \mathrm{E}-02$ \\
\hline Transcription Sirtuin6 regulation and functions & 3.062E-04 & 3.809E-02 \\
\hline $\begin{array}{l}\text { Role of ZNF202 in regulation of expression of genes involved in } \\
\text { atherosclerosis }\end{array}$ & 7.644E-04 & 7.149E-02 \\
\hline $\begin{array}{l}\text { Development Beta adrenergic receptors in brown adipocyte } \\
\text { differentiation }\end{array}$ & $8.592 \mathrm{E}-04$ & 7.149E-02 \\
\hline Expression targets of Tissue factor signaling in cancer & 9.195E-04 & 7.149E-02 \\
\hline$\overline{\text { Signal transduction mTORC1 downstream signaling }}$ & 1.456E-03 & 9.691E-02 \\
\hline Regulation of Tissue factor signaling in cancer & 1.719E-03 & $9.691 \mathrm{E}-02$ \\
\hline Processes & p-value & FDR \\
\hline lipid metabolic process & $1.500 \mathrm{E}-25$ & 9.695E-22 \\
\hline response to organic cyclic compound & 7.688E-24 & $2.484 \mathrm{E}-20$ \\
\hline response to oxygen-containing compound & 1.649E-23 & $3.553 \mathrm{E}-20$ \\
\hline single-organism metabolic process & $4.262 \mathrm{E}-23$ & $6.635 E-20$ \\
\hline small molecule metabolic process & $5.133 \mathrm{E}-23$ & $6.635 \mathrm{E}-20$ \\
\hline response to organic substance & 8.934E-21 & $9.623 \mathrm{E}-18$ \\
\hline response to external stimulus & $2.110 \mathrm{E}-20$ & $1.948 \mathrm{E}-17$ \\
\hline response to drug & 4.389E-20 & $3.546 \mathrm{E}-17$ \\
\hline steroid metabolic process & $5.788 \mathrm{E}-20$ & $3.908 \mathrm{E}-17$ \\
\hline single-organism process & $6.046 \mathrm{E}-20$ & $3.908 \mathrm{E}-17$ \\
\hline
\end{tabular}




\section{Table 43}

Enrichments for liver and metabolic disease endpoints in comparison CVvsC20

\begin{tabular}{|c|c|c|}
\hline Liver Toxicity Endpoint Processes & p-value & FDR \\
\hline Steatosis, development liver & 4.866E-02 & $5.422 \mathrm{E}-01$ \\
\hline Fibrosis, development liver & 7.746E-02 & $5.422 \mathrm{E}-01$ \\
\hline Hypertrophic organ growth liver & 1.679E-01 & 7.837E-01 \\
\hline Cell cycle, processes involved in G0-phase & $3.450 \mathrm{E}-01$ & $7.870 \mathrm{E}-01$ \\
\hline Cell cycle, processes involved in G1-phase & 4.245E-01 & $7.870 \mathrm{E}-01$ \\
\hline Cholestasis, development liver & 4.409E-01 & 7.870E-01 \\
\hline Cell cycle, processes involved in G2-phase & 4.702E-01 & $7.870 \mathrm{E}-01$ \\
\hline Peroxisomal proliferation, induction liver & 5.235E-01 & $7.870 \mathrm{E}-01$ \\
\hline Cell cycle, processes involved in S-phase & 5.474E-01 & 7.870E-01 \\
\hline Apoptosis via Mitochondrial membrane dysfunction & $6.746 \mathrm{E}-01$ & $7.870 \mathrm{E}-01$ \\
\hline Liver-Specific Maps & p-value & FDR \\
\hline Neurophysiological process Circadian rhythm & 1.030E-04 & 9.994E-03 \\
\hline DNA damage ATM/ATR regulation of G1/S checkpoint & 6.179E-04 & 2.997E-02 \\
\hline Development TGF-beta receptor signaling & 2.286E-03 & $6.552 \mathrm{E}-02$ \\
\hline Development WNT signaling pathway. Part 2 & $2.702 \mathrm{E}-03$ & $6.552 \mathrm{E}-02$ \\
\hline Signal transduction mTORC2 downstream signaling & $5.471 \mathrm{E}-03$ & 1.061E-01 \\
\hline Development Signaling of $\beta$-adrenergic receptors via Beta-arrestins & 7.044E-03 & 1.125E-01 \\
\hline DNA damage ATM / ATR regulation of G2 / M checkpoint & 8.240E-03 & 1.125E-01 \\
\hline Development SSTR1 in regulation of cell proliferation and migration & 1.019E-02 & 1.125E-01 \\
\hline DNA damage Brca1 as a transcription regulator & 1.088E-02 & 1.125E-01 \\
\hline $\begin{array}{l}\text { Regulation of lipid metabolism FXR-dependent negative-feedback } \\
\text { regulation of bile acids concentration }\end{array}$ & 1.159E-02 & $1.125 \mathrm{E}-01$ \\
\hline Nutritional and Metabolic Disease Biomarkers & p-value & FDR \\
\hline Hypokalemia & $6.052 \mathrm{E}-08$ & $3.752 \mathrm{E}-06$ \\
\hline$\overline{\text { Overnutrition }}$ & 9.254E-04 & 1.707E-02 \\
\hline Obesity & 9.254E-04 & 1.707E-02 \\
\hline Water-Electrolyte Imbalance & 1.105E-03 & 1.707E-02 \\
\hline Hypertriglyceridemia & 1.377E-03 & 1.707E-02 \\
\hline Hypercholesterolemia & 1.839E-03 & 1.901E-02 \\
\hline Hypoglycemia & $2.258 \mathrm{E}-03$ & $2.000 \mathrm{E}-02$ \\
\hline Hyperlipidemias & $2.774 \mathrm{E}-03$ & $2.150 \mathrm{E}-02$ \\
\hline Nutrition Disorders & 3.774E-03 & $2.600 \mathrm{E}-02$ \\
\hline Insulin Resistance & $7.968 \mathrm{E}-03$ & 4.448E-02 \\
\hline Nutritional and Metabolic Disease Maps & p-value & FDR \\
\hline Development Signaling of $\beta$-adrenergic receptors via Beta-arrestins & $2.670 \mathrm{E}-03$ & 9.095E-02 \\
\hline $\begin{array}{l}\text { Regulation of lipid metabolism FXR-dependent negative-feedback } \\
\text { regulation of bile acids concentration }\end{array}$ & 4.437E-03 & 9.095E-02 \\
\hline Bile acids regulation of glucose and lipid metabolism via FXR & 7.673E-03 & 9.830E-02 \\
\hline Transcription Androgen Receptor nuclear signaling & 9.590E-03 & $9.830 \mathrm{E}-02$ \\
\hline Development IGF-1 receptor signaling & 1.214E-02 & 9.958E-02 \\
\hline Unsaturated fatty acid biosynthesis & 1.699E-02 & 1.161E-01 \\
\hline Transcription Transcription regulation of aminoacid metabolism & 7.854E-02 & $2.751 \mathrm{E}-01$ \\
\hline Triacylglycerol metabolism p.2 & 8.456E-02 & $2.751 \mathrm{E}-01$ \\
\hline $\begin{array}{l}\text { Role of Diethylhexyl Phthalate and Tributyltin in fat cell } \\
\text { differentiation }\end{array}$ & $9.055 \mathrm{E}-02$ & 2.751E-01 \\
\hline Vitamin B6 metabolism & $9.353 \mathrm{E}-02$ & $2.751 \mathrm{E}-01$ \\
\hline
\end{tabular}


Table 44

Enrichments for liver and metabolic disease endpoints in comparison CVvsC200

\begin{tabular}{|c|c|c|}
\hline Liver Toxicity Endpoint Processes & p-value & FDR \\
\hline Ischemia-induced cellular changes liver & $1.588 \mathrm{E}-03$ & 2.179E-02 \\
\hline Hypoxia, development & 2.421E-03 & 2.179E-02 \\
\hline Apoptosis via Mitochondrial membrane dysfunction & 5.073E-02 & $2.521 \mathrm{E}-01$ \\
\hline Fibrosis, development liver & 7.399E-02 & 2.521E-01 \\
\hline Steatosis, development liver & 8.062E-02 & $2.521 \mathrm{E}-01$ \\
\hline Hypertrophic organ growth liver & 8.403E-02 & $2.521 \mathrm{E}-01$ \\
\hline Phospholipidosis, development liver & 1.391E-01 & 3.576E-01 \\
\hline Cholestasis, development liver & 1.710E-01 & 3.848E-01 \\
\hline Cell cycle, processes involved in G1-phase & 3.839E-01 & $7.678 \mathrm{E}-01$ \\
\hline Cell cycle, processes involved in G2-phase & 5.009E-01 & 8.667E-01 \\
\hline Liver-Specific Maps & p-value & FDR \\
\hline Immune response TLR2 and TLR4 signaling pathways & $1.658 \mathrm{E}-05$ & 5.109E-03 \\
\hline Apoptosis and survival p53-dependent apoptosis & 3.762E-05 & 5.109E-03 \\
\hline G-protein signaling Proinsulin C-peptide signaling & 5.040E-05 & 5.109E-03 \\
\hline Signal transduction mTORC2 upstream signaling & 5.539E-05 & 5.109E-03 \\
\hline $\begin{array}{l}\text { Apoptosis and survival Endoplasmic reticulum stress response } \\
\text { pathway }\end{array}$ & 9.226E-05 & $5.608 \mathrm{E}-03$ \\
\hline Immune response IL-1 signaling pathway & 9.229E-05 & $5.608 \mathrm{E}-03$ \\
\hline Immune response TLR5, TLR7, TLR8, TLR9 signaling pathways & 1.064E-04 & $5.608 \mathrm{E}-03$ \\
\hline Immune response IL-18 signaling & 1.600E-04 & 7.170E-03 \\
\hline Histamine $\mathrm{H} 1$ receptor signaling/immune response & 1.749E-04 & 7.170E-03 \\
\hline Development ERBB-family signaling & 2.779E-04 & $9.580 \mathrm{E}-03$ \\
\hline Nutritional and Metabolic Disease Biomarkers & p-value & FDR \\
\hline Obesity & $3.371 \mathrm{E}-07$ & $2.174 \mathrm{E}-05$ \\
\hline Overnutrition & 3.371E-07 & 2.174E-05 \\
\hline Nutrition Disorders & 3.925E-06 & $1.688 \mathrm{E}-04$ \\
\hline Vascular Calcification & 9.711E-05 & $3.132 \mathrm{E}-03$ \\
\hline Frontotemporal Dementia & $5.455 \mathrm{E}-04$ & 1.173E-02 \\
\hline Frontotemporal Lobar Degeneration & $5.455 \mathrm{E}-04$ & 1.173E-02 \\
\hline Hyperinsulinism & 9.153E-04 & 1.687E-02 \\
\hline Diabetes Mellitus, Type 1 & 1.805E-03 & $2.911 \mathrm{E}-02$ \\
\hline Insulin Resistance & 4.129E-03 & $5.918 \mathrm{E}-02$ \\
\hline Calcinosis & 5.304E-03 & $6.842 \mathrm{E}-02$ \\
\hline Nutritional and Metabolic Disease Maps & p-value & FDR \\
\hline G-protein signaling Proinsulin C-peptide signaling & 1.347E-06 & $2.102 \mathrm{E}-04$ \\
\hline Development Activation of ERK by Alpha-1 adrenergic receptors & 4.336E-06 & $2.431 \mathrm{E}-04$ \\
\hline Immune response IL-18 signaling & 4.675E-06 & $2.431 \mathrm{E}-04$ \\
\hline Development IGF-1 receptor signaling & 1.332E-05 & 5.196E-04 \\
\hline Putative pathways for BPA-stimulated fat cell differentiation & 5.286E-05 & 1.649E-03 \\
\hline Apoptosis and survival Ceramides signaling pathway & 1.942E-04 & 5.049E-03 \\
\hline $\begin{array}{l}\text { Development } \beta \text { adrenergic receptors in brown adipocyte } \\
\text { differentiation }\end{array}$ & 1.109E-03 & 2.471E-02 \\
\hline Transcription Transcription regulation of aminoacid metabolism & 1.875E-03 & $3.656 \mathrm{E}-02$ \\
\hline Transcription Sirtuin6 regulation and functions & 2.481E-03 & $4.221 \mathrm{E}-02$ \\
\hline Membrane-bound ESR1: interaction with growth factors signaling & 2.706E-03 & $4.221 \mathrm{E}-02$ \\
\hline
\end{tabular}


Table 45

Enrichments for liver and metabolic disease endpoints in comparison $\mathrm{HVvsH} 20$

\begin{tabular}{|c|c|c|}
\hline Liver Toxicity Endpoint Processes & p-value & FDR \\
\hline Cholestasis, development liver & $6.523 \mathrm{E}-04$ & $9.480 \mathrm{E}-03$ \\
\hline Steatosis, development liver & 1.053E-03 & $9.480 \mathrm{E}-03$ \\
\hline Fibrosis, development liver & 1.364E-02 & $6.576 \mathrm{E}-02$ \\
\hline Progression of oxidative stress & 1.461E-02 & $6.576 \mathrm{E}-02$ \\
\hline Inflammation, development & $2.248 \mathrm{E}-02$ & $8.092 \mathrm{E}-02$ \\
\hline Hypertrophic organ growth liver & 1.108E-01 & 3.323E-01 \\
\hline Hypoxia, development & 4.259E-01 & $1.000 \mathrm{E}+00$ \\
\hline Apoptosis via Mitochondrial membrane dysfunction & 5.346E-01 & $1.000 \mathrm{E}+00$ \\
\hline Ischemia-induced cellular changes liver & 8.177E-01 & $1.000 \mathrm{E}+00$ \\
\hline Apoptosis via Death Domain receptors cascades & $8.298 \mathrm{E}-01$ & $1.000 \mathrm{E}+00$ \\
\hline Liver-Specific Maps & p-value & FDR \\
\hline IL-6-induced acute-phase response/hepatocytes & $4.661 \mathrm{E}-13$ & $1.571 \mathrm{E}-10$ \\
\hline Immune response IL-6 signaling pathway via JAK/STAT & 9.310E-07 & 1.569E-04 \\
\hline Signal transduction mTORC2 downstream signaling & $2.422 \mathrm{E}-05$ & $2.721 \mathrm{E}-03$ \\
\hline MIF-induced cell adhesion, migration and angiogenesis & 4.658E-05 & $3.231 \mathrm{E}-03$ \\
\hline Development Ligand-dependent activation-ESR1/AP-1 pathway & 4.793E-05 & $3.231 \mathrm{E}-03$ \\
\hline Development Beta-adrenergic receptors transactivation of EGFR & 8.020E-05 & 4.505E-03 \\
\hline Transcription Role of AP-1 in regulation of cellular metabolism & 9.590E-05 & 4.617E-03 \\
\hline Glutathione metabolism & $1.810 \mathrm{E}-04$ & $7.624 \mathrm{E}-03$ \\
\hline Development VEGF signaling and activation & 2.163E-04 & 8.100E-03 \\
\hline Immune response IL-1 signaling pathway & $2.509 \mathrm{E}-04$ & 8.457E-03 \\
\hline Nutritional and Metabolic Disease Biomarkers & p-value & FDR \\
\hline Nutrition Disorders & 9.404E-08 & $7.284 \mathrm{E}-06$ \\
\hline Obesity & 1.539E-07 & $7.284 \mathrm{E}-06$ \\
\hline Overnutrition & 1.539E-07 & $7.284 \mathrm{E}-06$ \\
\hline Diabetes Mellitus, Type 2 & 1.879E-06 & 6.669E-05 \\
\hline Dyslipidemias & 6.992E-06 & 1.986E-04 \\
\hline Hypokalemia & 2.684E-05 & $6.352 \mathrm{E}-04$ \\
\hline Hyperinsulinism & $2.901 \mathrm{E}-04$ & $5.885 \mathrm{E}-03$ \\
\hline Lipid Metabolism Disorders & 4.613E-04 & $8.188 \mathrm{E}-03$ \\
\hline Colorectal Neoplasms, Hereditary Nonpolyposis & 6.567E-04 & 1.036E-02 \\
\hline Hyperlipidemias & 1.077E-03 & $1.472 \mathrm{E}-02$ \\
\hline Nutritional and Metabolic Disease Maps & p-value & FDR \\
\hline Ligand-dependent activation of the ESR1/AP-1 pathway & 1.295E-05 & 7.881E-04 \\
\hline$\underline{\beta}$ adrenergic receptors/brown adipocyte differentiation & 1.397E-05 & $7.881 \mathrm{E}-04$ \\
\hline Transcription Role of AP-1 in regulation of cellular metabolism & 1.681E-05 & $7.881 \mathrm{E}-04$ \\
\hline Glutathione metabolism & 2.174E-05 & 7.881E-04 \\
\hline $\begin{array}{l}\text { Putative pathways for stimulation of fat cell differentiation by } \\
\text { Bisphenol A }\end{array}$ & 6.243E-05 & 1.811E-03 \\
\hline Immune response IL-18 signaling & $3.418 \mathrm{E}-04$ & $8.259 \mathrm{E}-03$ \\
\hline Transcription Sirtuin6 regulation and functions & 5.104E-04 & 1.057E-02 \\
\hline Development $\alpha-1$ adrenergic receptors signaling via Cyclic AMP & 7.107E-04 & $1.288 \mathrm{E}-02$ \\
\hline Insulin, IGF-1 and TNF-alpha in brown adipocyte differentiation & 9.726E-04 & 1.567E-02 \\
\hline $\begin{array}{l}\text { Regulation of metabolism Bile acids regulation of glucose and lipid } \\
\text { metabolism via FXR }\end{array}$ & 2.027E-03 & $2.939 \mathrm{E}-02$ \\
\hline \multicolumn{3}{|c|}{$\begin{array}{l}\text { Enrichment is based on the significantly differentially transcribed genes }(p<.05) \text { in a } \\
\text { comparison between control diet/vehicle and control diet/20 mg/kg PCB }(C V v s C 20) \\
\text { enrichments for each grouping, ranked from most to least significant by } p \text {-value }\end{array}$} \\
\hline
\end{tabular}


Table 46

Enrichments for liver and metabolic disease endpoints in comparison HVvsH200

\begin{tabular}{|c|c|c|}
\hline Liver Toxicity Endpoint Processes & p-value & FDR \\
\hline Hypertrophic organ growth liver & $1.768 \mathrm{E}-03$ & $2.109 \mathrm{E}-02$ \\
\hline Fibrosis, development liver & $2.343 \mathrm{E}-03$ & $2.109 \mathrm{E}-02$ \\
\hline Steatosis, development liver & 5.006E-03 & 3.004E-02 \\
\hline Cell cycle progression of Mitosis & $8.310 \mathrm{E}-02$ & 3.725E-01 \\
\hline Apoptosis via Mitochondrial membrane dysfunction & 1.035E-01 & 3.725E-01 \\
\hline Cholestasis, development liver & 1.580E-01 & 4.741E-01 \\
\hline Hypoxia, development & 3.809E-01 & 9.793E-01 \\
\hline Inflammation, development & 4.762E-01 & 9.971E-01 \\
\hline Cell cycle, processes involved in S-phase & 5.009E-01 & 9.971E-01 \\
\hline Cell cycle, processes involved in G1-phase & $6.586 \mathrm{E}-01$ & 9.971E-01 \\
\hline Liver-Specific Maps & p-value & FDR \\
\hline Development Regulation of EMT & 3.347E-06 & 8.870E-04 \\
\hline Immune response IL-6-induced acute-phase response in & & \\
\hline hepatocytes & $1.092 \mathrm{E}-05$ & $1.447 \mathrm{E}-03$ \\
\hline Immune response Oncostatin M signaling via JAK-Stat & 2.479E-04 & $2.190 \mathrm{E}-02$ \\
\hline$\overline{\text { Signal transduction mTORC2 downstream signaling }}$ & 4.219E-04 & $2.795 \mathrm{E}-02$ \\
\hline Development IGF-1 receptor signaling & 8.743E-04 & $4.215 \mathrm{E}-02$ \\
\hline Development WNT signaling pathway. Part 2 & 9.544E-04 & $4.215 \mathrm{E}-02$ \\
\hline Development Ligand-dependent activation - ESR1/AP-1 pathway & 1.180E-03 & 4.469E-02 \\
\hline Cell cycle ESR1 regulation of G1/S transition & 1.376E-03 & $4.557 \mathrm{E}-02$ \\
\hline Development Beta-adrenergic receptors transactivation of EGFR & 1.895E-03 & $5.580 \mathrm{E}-02$ \\
\hline Transcription Sirtuin6 regulation and functions & $2.236 \mathrm{E}-03$ & $5.926 \mathrm{E}-02$ \\
\hline Nutritional and Metabolic Disease Biomarkers & p-value & FDR \\
\hline Obesity & $8.546 \mathrm{E}-05$ & 2.606E-03 \\
\hline Overnutrition & 8.546E-05 & $2.606 \mathrm{E}-03$ \\
\hline Vascular Calcification & 1.017E-04 & $2.606 \mathrm{E}-03$ \\
\hline Nutrition Disorders & 1.032E-04 & $2.606 \mathrm{E}-03$ \\
\hline Frontotemporal Dementia & 1.580E-04 & $2.659 \mathrm{E}-03$ \\
\hline Frontotemporal Lobar Degeneration & 1.580E-04 & $2.659 \mathrm{E}-03$ \\
\hline Zellweger Syndrome & 1.506E-03 & $2.165 \mathrm{E}-02$ \\
\hline Peroxisome biogenesis disorders & 1.715E-03 & $2.165 \mathrm{E}-02$ \\
\hline$\overline{\text { Diabetes Mellitus, Type } 2}$ & $2.031 \mathrm{E}-03$ & $2.279 \mathrm{E}-02$ \\
\hline Calcinosis & $2.557 \mathrm{E}-03$ & $2.582 \mathrm{E}-02$ \\
\hline$\overline{\text { Nutritional and Metabolic Disease Maps }}$ & p-value & FDR \\
\hline Development IGF-1 receptor signaling & $2.504 \mathrm{E}-04$ & $2.165 \mathrm{E}-02$ \\
\hline Development Ligand-dependent activation - ESR1/AP-1 pathway & $5.388 \mathrm{E}-04$ & $2.165 \mathrm{E}-02$ \\
\hline Transcription Sirtuin6 regulation and functions & 6.629E-04 & $2.165 \mathrm{E}-02$ \\
\hline Retinol metabolism & 1.066E-03 & $2.612 \mathrm{E}-02$ \\
\hline Unsaturated fatty acid biosynthesis & 4.752E-03 & 9.314E-02 \\
\hline Galactose metabolism & $6.265 \mathrm{E}-03$ & 1.023E-01 \\
\hline $\begin{array}{l}\text { Regulation of metabolism Bile acids regulation of glucose and lipid } \\
\text { metabolism via FXR }\end{array}$ & $1.028 \mathrm{E}-02$ & $1.439 \mathrm{E}-01$ \\
\hline Transcription Androgen Receptor nuclear signaling & 1.407E-02 & 1.724E-01 \\
\hline Signal transduction PTMs in BAFF-induced signaling & 1.857E-02 & $2.022 \mathrm{E}-01$ \\
\hline Amitraz-induced inhibition of Insulin secretion & $2.424 \mathrm{E}-02$ & 2.127E-01 \\
\hline
\end{tabular}


Table 47

Targets associated with liver disease enrichments and change vs. vehicle control by comparison

\begin{tabular}{|c|c|c|c|c|c|}
\hline Input IDs & $\begin{array}{l}\text { Gene } \\
\text { Symbol }\end{array}$ & CVvsC20 & CVvsC200 & HVvsH2O & HVvsH2OO \\
\hline \multicolumn{6}{|l|}{ Liver toxicity: Steatosis } \\
\hline ENSMUSG00000035561 & Aldh1b1 & & & -0.68 & \\
\hline ENSMUSG00000025003 & Cyp2c39 & & & -2.02 & \\
\hline ENSMUSG00000066072 & Cyp4a10 & & & -0.83 & \\
\hline ENSMUSG00000032349 & Elovl5 & & & -0.53 & \\
\hline ENSMUSG00000010663 & Fads1 & & & & -0.64 \\
\hline ENSMUSG00000025153 & Fasn & & & -1.12 & \\
\hline ENSMUSG00000078650 & $\mathrm{G} 6 \mathrm{pc}$ & & & & 1.01 \\
\hline ENSMUSG00000027984 & $\underline{\mathrm{Hadh}}$ & & & -0.47 & \\
\hline ENSMUSG00000042632 & Pla2g6 & & 0.98 & & \\
\hline ENSMUSG00000023913 & Pla2g7 & & 0.65 & & \\
\hline ENSMUSG00000000440 & Pparg & & 0.62 & & \\
\hline \multicolumn{6}{|l|}{ Liver toxicity: fibrosis } \\
\hline ENSMUSG00000000532 & Acvr1b & & 0.97 & & \\
\hline ENSMUSG00000041324 & Inhba & & & 1.24 & \\
\hline ENSMUSG00000039304 & Tnfsf10 & & 1.10 & & \\
\hline ENSMUSG00000029661 & Col1a2 & -0.76 & & & \\
\hline ENSMUSG00000021820 & Camk2g & & 0.80 & & \\
\hline ENSMUSG00000029675 & Eln & & & & -0.87 \\
\hline ENSMUSG00000026072 & IIr1 & & & 0.58 & \\
\hline ENSMUSG00000017057 & Il13ra1 & & & 0.71 & \\
\hline ENSMUSG00000030748 & II4ra & & 0.57 & & \\
\hline ENSMUSG00000027947 & $116 \mathrm{ra}$ & & & -0.67 & \\
\hline ENSMUSG00000029304 & Spp1 & & & 0.48 & \\
\hline ENSMUSG00000024620 & Pdgfrb & & & & -0.53 \\
\hline ENSMUSG00000028599 & $\underline{\text { Tnfrsf1b }}$ & & & & 0.58 \\
\hline ENSMUSG00000021250 & Fos & & & 1.27 & \\
\hline
\end{tabular}




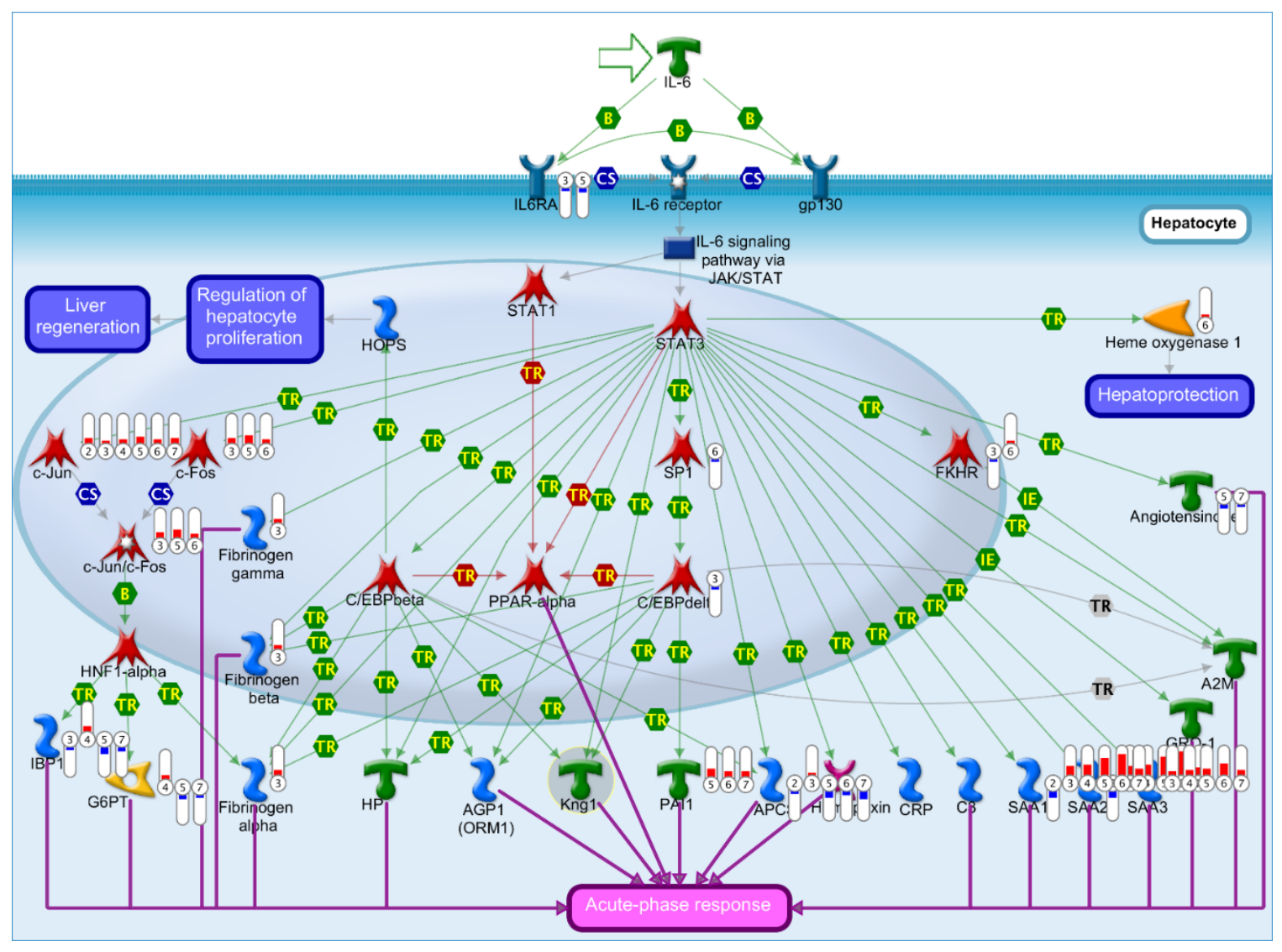

Figure 11. IL-6-mediated acute-phase response in hepatocytes.

PCB exposure and PCB/HFD coexposures induced transcriptional changes in elements of IL-6mediated acute-phase response in hepatocytes. In particular, components of the AP-1 complex (c-Jun, c-Fos) were differentially regulated in the CVvsC200 comparison (2), the HVvsH2O comparison (3), and the HVvsH200 comparison (4), along with changes in transcription of HNF1alpha targets IBP1, G6PT and Fibrinogen alpha. The HVvsH20 condition was associated with differential transcription of the most targets associated with this pathway, in particular, targets of STAT3 transcriptional activation. An increase in local inflammation associated with this condition is consistent with our histological findings. Image generated using MetaCore ${ }^{\mathrm{TM}}$ from Thomson Reuters. 


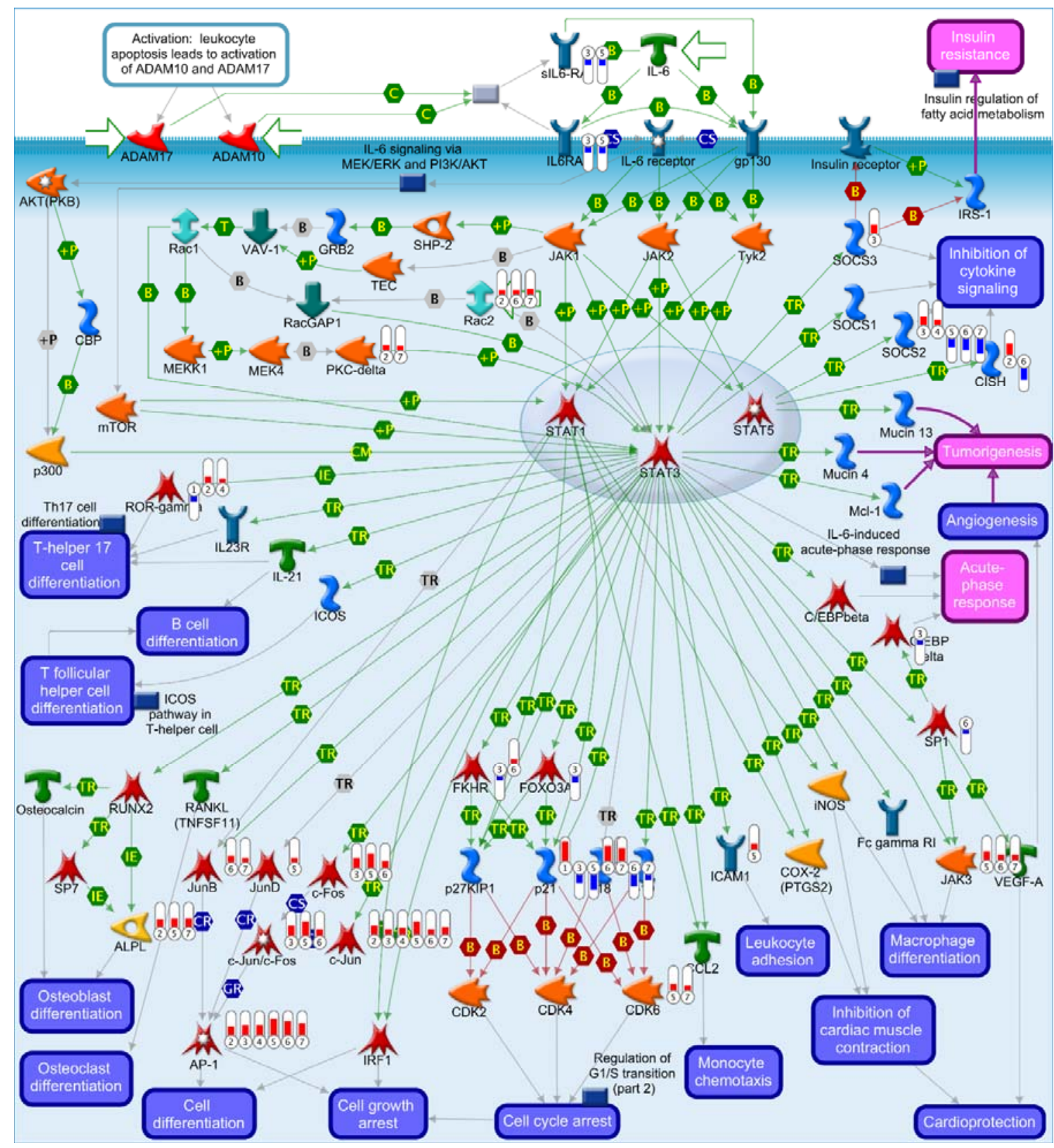

Figure 12. IL-6/STAT-3 signaling pathways.

PCB exposure and PCB/HFD coexposures induced transcriptional changes in elements of IL-6signaling pathways. In particular, components of the AP-1 complex (c-Jun, c-Fos) were differentially regulated in the CVvsC200 comparison (2), the $\mathrm{HVvsH} 20$ comparison (3), and the $\mathrm{HVvsH} 200$ comparison (4). Image generated using MetaCore ${ }^{\mathrm{TM}}$ from Thomson Reuters. 
Both the lack of transcription-level changes in biomarkers of liver disease in the CVvsC20 comparison and the relative abundance of changes in the $\mathrm{HVvsH} 20$ comparison vs. either of the higher PCB exposure groups is in keeping with the histological severity of liver disease in these groups $^{23}$. Few common targets were seen in this enrichment, which may suggest pleiotropic effects on hepatocyte toxicity, as well as complex interactions between nutrition/PCB coexposures on disease mechanisms.

Among nutritional and metabolic disease enrichments, the pattern presented by the individual comparisons was far more cohesive, with over-nutrition/obesity in the top enrichments for every comparison. Fold changes of differentially transcribed genes associated with overnutrition/obesity for each vehicle vs. PCB comparison are listed in Table 48. Other metabolic diseases that were of interest to us and highly enriched in one or more datasets were type 2 diabetes mellitus (Table 49), metabolic syndrome (Table 50), and insulin resistance (Table 51). In each of these disease categories, the condition with the most changes vs. vehicle control was the $\mathrm{HVvsH} 20$ condition. These changes included down-regulation of fatty acid synthetase and beta 2-adrenergic receptor, polymorphisms of which have been associated with metabolic disease $^{123,124}$. Down-regulation of GLUT4 reduces insulin-sensitive glucose uptake in nonhepatocyte liver cells and other insulin-sensitive tissues ${ }^{125}$. Metabolic disease enrichments for the CVvsC20 and CVvsC200 group indicated changes to PPAR-alpha and LXR-mediated glucose and lipid homeostasis, including alterations in expressions of the Tribbles-3 pseudokinase, which contributes to insulin resistance ${ }^{126}$ and down-regulation of Cyp7a1, which could lead to cholesterol accumulation.

MetaCore ${ }^{\mathrm{TM}}$ network analysis of dose-within-diet comparisons did not appear to indicate enrichment in transcriptional targets of the xenobiotic transcription factors $A h R, P X R$, or CAR

Because xenobiotic receptors AhR, PXR, and CAR are classically associated with transcriptional response to PCBs and other chemicals, we used MetaCore ${ }^{\mathrm{TM}}$ to evaluate overrepresentation of curated transcriptional targets of these receptors. The direct transcriptional targets of these receptors as defined by the MetaCore ${ }^{\mathrm{TM}}$ database did not comprise a large 
Table 48

Targets associated with metabolic disease enrichments (overnutrition/obesity) and their change vs. vehicle control in each comparison

\begin{tabular}{|c|c|c|c|c|c|}
\hline Input IDs & $\begin{array}{l}\text { Gene } \\
\text { Symbol }\end{array}$ & CVvsC20 & CVvsC200 & HVvsH20 & HVvsH2OO \\
\hline \multicolumn{6}{|c|}{ Metabolic Disease Biomarkers: Obesity } \\
\hline ENSMUSG00000020917 & Acly & & & -0.72 & \\
\hline ENSMUSG00000031278 & Acsl4 & & -0.47 & 1.05 & \\
\hline ENSMUSG00000035783 & Acta2 & & -0.96 & & -0.71 \\
\hline ENSMUSG00000026883 & Dab2ip & & 0.65 & & \\
\hline ENSMUSG00000042429 & Adora1 & & 0.81 & -0.64 & -0.66 \\
\hline ENSMUSG00000022994 & Adcy6 & & & -0.69 & \\
\hline ENSMUSG00000005580 & Adcy9 & & & -0.92 & -0.73 \\
\hline ENSMUSG00000046532 & $\mathrm{Ar}$ & & -0.86 & -1.11 & \\
\hline ENSMUSG00000027792 & Bche & & & -0.67 & \\
\hline ENSMUSG00000055116 & Arntl & 2.19 & 1.04 & & \\
\hline ENSMUSG00000045730 & Adrb2 & -1.04 & & -1.49 & \\
\hline ENSMUSG00000082361 & Btc & & 1.10 & & \\
\hline ENSMUSG00000002944 & $\mathrm{Cd} 36$ & -0.75 & 0.74 & -0.89 & \\
\hline ENSMUSG00000039804 & Ncoa5 & & -0.65 & & \\
\hline ENSMUSG00000029238 & Clock & 0.77 & & & \\
\hline ENSMUSG00000000326 & Comt & & & 0.62 & \\
\hline ENSMUSG00000056054 & S100a8 & & 1.44 & 1.49 & 1.62 \\
\hline ENSMUSG00000056071 & S100a9 & & 1.39 & 1.69 & 1.58 \\
\hline ENSMUSG00000019768 & Esr1 & & & 0.57 & 0.69 \\
\hline ENSMUSG00000025153 & Fasn & & & -1.12 & \\
\hline ENSMUSG00000044167 & $\overline{\text { Foxo1 }}$ & & & -0.53 & \\
\hline ENSMUSG00000048756 & Foxo3 & & & -0.79 & \\
\hline ENSMUSG00000019779 & Frk & & 0.58 & & \\
\hline ENSMUSG00000028001 & $\overline{\text { Fga }}$ & & & 1.06 & \\
\hline ENSMUSG00000033831 & $\overline{F g b}$ & & & 1.02 & \\
\hline ENSMUSG00000033860 & Fgg & & & 0.96 & \\
\hline ENSMUSG00000018566 & Slc2a4 & & & -1.78 & \\
\hline ENSMUSG00000031451 & Gas6 & & & -0.47 & \\
\hline ENSMUSG00000091971 & Hspa1a & & -3.96 & & \\
\hline ENSMUSG00000041798 & Gck & & -0.98 & -1.01 & -0.63 \\
\hline ENSMUSG00000020429 & Igfbp1 & & & -0.46 & 1.08 \\
\hline ENSMUSG00000020427 & Igfbp3 & & 0.44 & & \\
\hline ENSMUSG00000046070 & Igfals & & -0.70 & & \\
\hline ENSMUSG00000027947 & II6ra & & & -0.67 & \\
\hline ENSMUSG00000038894 & $\underline{\operatorname{Irs2}}$ & & & 0.57 & 0.83 \\
\hline ENSMUSG00000025780 & Itih5 & & 0.60 & 1.01 & \\
\hline ENSMUSG00000055148 & $\underline{\text { Klf2 }}$ & & -1.38 & -0.74 & \\
\hline ENSMUSG00000032796 & Lama1 & & & -0.63 & \\
\hline ENSMUSG00000053846 & Lipg & -1.08 & -0.78 & -1.66 & -0.94 \\
\hline ENSMUSG00000020593 & Lpin1 & & -0.76 & -1.96 & -0.70 \\
\hline
\end{tabular}


Table 48 (continued)

Targets associated with metabolic disease enrichments (overnutrition/obesity) and their change vs. vehicle control in each comparison

\begin{tabular}{|c|c|c|c|c|c|}
\hline Input IDs & $\begin{array}{l}\text { Gene } \\
\text { Symbol }\end{array}$ & CVvsC20 & CVvsC200 & HVvsH20 & HVvsH2OO \\
\hline ENSMUSG00000028553 & Angptl3 & & & -0.55 & \\
\hline ENSMUSG00000061132 & Blnk & -1.59 & & & \\
\hline ENSMUSG00000027556 & Car1 & & -0.65 & & \\
\hline ENSMUSG00000008845 & Cd163 & 0.84 & 1.32 & & \\
\hline ENSMUSG00000028551 & Cdkn2c & & 0.93 & & \\
\hline ENSMUSG00000020038 & Cry1 & & & & 0.65 \\
\hline ENSMUSG00000068742 & Cry2 & & 0.48 & & \\
\hline ENSMUSG00000015312 & Gadd45b & 2.17 & 1.23 & & 1.05 \\
\hline ENSMUSG00000038039 & Gcc2 & & 0.69 & & \\
\hline ENSMUSG00000026864 & $\overline{\mathrm{Hspa5}}$ & & -1.17 & & \\
\hline ENSMUSG00000074896 & Ifit3 & & 1.13 & & 0.67 \\
\hline ENSMUSG00000052684 & $\overline{\text { Jun }}$ & & 1.15 & 0.62 & 0.80 \\
\hline ENSMUSG00000049723 & Mmp12 & & & -0.97 & \\
\hline ENSMUSG00000003849 & Nqo1 & & 0.47 & -1.28 & \\
\hline ENSMUSG00000020889 & $\mathrm{Nr1d1}$ & 1.04 & & & -1.52 \\
\hline ENSMUSG00000020893 & $\overline{\text { Per1 }}$ & & & -0.97 & \\
\hline ENSMUSG00000025509 & Pnpla2 & & 0.85 & & \\
\hline ENSMUSG00000045038 & Prkce & & & -0.53 & \\
\hline ENSMUSG00000020641 & Rsad2 & & 0.89 & & 0.72 \\
\hline ENSMUSG00000057465 & Saa2 & & -1.37 & 2.06 & 2.62 \\
\hline ENSMUSG00000041567 & Serpina12 & & -1.04 & 0.65 & \\
\hline ENSMUSG00000032902 & SIc16a1 & & & 0.59 & \\
\hline ENSMUSG00000020027 & Socs2 & & & 1.10 & 0.71 \\
\hline ENSMUSG00000023905 & Tnfrsf12a & & & 0.81 & \\
\hline ENSMUSG00000034485 & Uaca & & 0.69 & & \\
\hline ENSMUSG00000008348 & Ubc & & -0.65 & & \\
\hline ENSMUSG00000024924 & $\overline{\text { Vldlr }}$ & & & -2.54 & \\
\hline $\begin{array}{l}\text { Metabolic disease analysi } \\
\text { including obesity/overnutri } \\
\text { comparison, fold change }\end{array}$ & or each com & $\begin{array}{l}\text { rison indicat } \\
\text { y regulated } \\
\text { vn. }\end{array}$ & ed gene sets & associated w & $\begin{array}{l}\text { th endpoints } \\
\text { For each }\end{array}$ \\
\hline
\end{tabular}


Table 48 (continued)

Targets associated with metabolic disease enrichments (overnutrition/obesity) and their change vs. vehicle control in each comparison

\begin{tabular}{|c|c|c|c|c|c|}
\hline Input IDs & $\begin{array}{l}\text { Gene } \\
\text { Symbol }\end{array}$ & CVvsC20 & CVvsC200 & HVvsH20 & HVvsH200 \\
\hline ENSMUSG00000024052 & Lpin2 & & & -0.73 & \\
\hline ENSMUSG00000015568 & $\underline{\mathrm{Lpl}}$ & & 0.72 & 0.91 & \\
\hline ENSMUSG00000027253 & Lrp4 & -0.73 & & -0.53 & \\
\hline ENSMUSG00000040584 & $\overline{\mathrm{Abcb} 1 \mathrm{a}}$ & & & -2.05 & \\
\hline ENSMUSG00000024589 & Nedd4I & 1.14 & 0.60 & 0.86 & \\
\hline ENSMUSG00000026822 & Lcn2 & 1.18 & & & 1.47 \\
\hline ENSMUSG00000032715 & Trib3 & 1.21 & 0.91 & -0.59 & \\
\hline ENSMUSG00000024413 & Npc1 & & 0.68 & & \\
\hline ENSMUSG00000030659 & Nucb2 & & & 0.82 & \\
\hline ENSMUSG00000029304 & Spp1 & & & 0.48 & \\
\hline ENSMUSG00000038508 & Gdf15 & & 1.52 & 1.22 & 1.08 \\
\hline ENSMUSG00000055866 & Per2 & -1.39 & -0.74 & & \\
\hline ENSMUSG00000033871 & Ppargc1b & & 0.86 & & \\
\hline ENSMUSG00000002289 & Angpt|4 & & -0.56 & -0.48 & \\
\hline ENSMUSG00000032462 & Pik3cb & & 0.89 & & \\
\hline ENSMUSG00000041417 & Pik3r1 & & -0.47 & & \\
\hline ENSMUSG00000000440 & Pparg & & 0.62 & & \\
\hline ENSMUSG00000027540 & Ptpn1 & & & 0.61 & \\
\hline ENSMUSG00000021876 & Rnase4 & & & 0.66 & \\
\hline ENSMUSG00000022883 & Robo1 & -0.80 & & -1.82 & \\
\hline ENSMUSG00000028150 & $\overline{\text { Rorc }}$ & -0.74 & 0.88 & & 0.53 \\
\hline ENSMUSG00000074115 & Saa1 & & -1.44 & 2.34 & 2.41 \\
\hline ENSMUSG00000075701 & Selenos & & -0.47 & & \\
\hline ENSMUSG00000060807 & Serpina6 & -0.97 & & -1.02 & \\
\hline ENSMUSG00000053113 & Socs3 & & & 1.08 & \\
\hline ENSMUSG00000025006 & Sorbs1 & & 0.64 & -0.50 & \\
\hline ENSMUSG00000012428 & $\overline{\text { Steap4 }}$ & & -0.62 & 1.22 & \\
\hline ENSMUSG00000025743 & Sdc3 & & 0.49 & & \\
\hline ENSMUSG00000029174 & $\overline{\mathrm{Tbc} 1 \mathrm{~d} 1}$ & & 0.57 & & \\
\hline ENSMUSG00000028599 & Tnfrsf1b & & & & 0.58 \\
\hline ENSMUSG00000035799 & Twist1 & & 1.70 & & \\
\hline ENSMUSG00000055254 & Ntrk2 & -1.28 & -2.03 & & 0.72 \\
\hline ENSMUSG00000027962 & Vcam1 & & 0.63 & -0.85 & \\
\hline ENSMUSG00000020484 & $\underline{X b p 1}$ & & & 0.71 & \\
\hline ENSMUSG00000028978 & Nos3 & & -0.87 & & \\
\hline ENSMUSG00000041653 & Pnpla3 & & & -1.78 & -1.17 \\
\hline ENSMUSG00000034853 & Acot11 & & 1.52 & & \\
\hline ENSMUSG00000004730 & Adgre1 & & 0.80 & & \\
\hline
\end{tabular}


Table 49

Targets associated with metabolic disease enrichments (Type 2 Diabetes Mellitus) and their change vs. vehicle control in each comparison

\begin{tabular}{|c|c|c|c|c|c|}
\hline Input IDs & Gene Symbol & CVvsC20 & CVvsC200 & HVvsH20 & HVvsH200 \\
\hline \multicolumn{6}{|c|}{ Metabolic Disease Biomarkers: Type 2 Diabetes Mellitus } \\
\hline ENSMUSG00000035783 & Acta2 & & -0.96 & & -0.71 \\
\hline ENSMUSG00000026883 & Dab2ip & & 0.65 & & \\
\hline ENSMUSG00000035561 & Aldh1b1 & & & -0.68 & \\
\hline ENSMUSG00000026289 & Atg16l1 & & -0.57 & & \\
\hline ENSMUSG00000024391 & Apom & & & 0.76 & \\
\hline ENSMUSG00000019947 & Arid5b & & & & 0.51 \\
\hline ENSMUSG00000046532 & $\underline{\underline{\mathrm{Ar}}}$ & & -0.86 & -1.11 & \\
\hline ENSMUSG00000032066 & Bco2 & & & -0.45 & \\
\hline ENSMUSG00000027792 & Bche & & & -0.67 & \\
\hline ENSMUSG00000055116 & Arntl & 2.19 & 1.04 & & \\
\hline ENSMUSG00000033863 & Klf9 & 0.91 & 0.80 & & \\
\hline ENSMUSG00000045730 & Adrb2 & -1.04 & & -1.49 & \\
\hline ENSMUSG00000082361 & Btc & & 1.10 & & \\
\hline ENSMUSG00000027381 & Bcl2/11 & & & & 0.91 \\
\hline ENSMUSG00000022637 & Cblb & & 0.62 & & \\
\hline ENSMUSG00000035042 & $\mathrm{Ccl} 5$ & & & -0.97 & \\
\hline ENSMUSG00000002944 & Cd36 & -0.75 & 0.74 & -0.89 & \\
\hline ENSMUSG00000039804 & Ncoa5 & & -0.65 & & \\
\hline ENSMUSG00000000326 & Comt & & & 0.62 & \\
\hline ENSMUSG00000068742 & Cry2 & & 0.48 & & \\
\hline ENSMUSG00000019997 & Ctgf & & & -0.85 & -0.63 \\
\hline ENSMUSG00000025003 & Cyp2c39 & & & -2.02 & \\
\hline ENSMUSG00000003053 & Сур2c29 & & 2.75 & & 1.31 \\
\hline ENSMUSG00000056054 & S100a8 & & 1.44 & 1.49 & 1.62 \\
\hline ENSMUSG00000056071 & S100a9 & & 1.39 & 1.69 & 1.58 \\
\hline ENSMUSG00000028914 & Casp9 & & 0.64 & & \\
\hline ENSMUSG00000072082 & Ccnf & & & 0.46 & \\
\hline ENSMUSG00000027533 & Fabp5 & & & 0.80 & \\
\hline ENSMUSG00000054191 & Klf1 & & & -0.73 & \\
\hline ENSMUSG00000041112 & Elmo1 & & & & 0.87 \\
\hline ENSMUSG00000042787 & Exog & & -0.92 & & -0.88 \\
\hline ENSMUSG00000019768 & Esr1 & & & 0.57 & 0.69 \\
\hline ENSMUSG00000010663 & Fads1 & & & & -0.64 \\
\hline ENSMUSG00000039529 & Atp8b1 & & & 0.42 & \\
\hline ENSMUSG00000044167 & Foxo1 & & & -0.53 & \\
\hline ENSMUSG00000048756 & Foxo3 & & & -0.79 & \\
\hline ENSMUSG00000049721 & Gal3st1 & & 1.51 & & \\
\hline ENSMUSG00000029992 & Gfpt1 & & -0.75 & & -0.56 \\
\hline ENSMUSG00000028645 & Slc2a1 & & 0.93 & & \\
\hline ENSMUSG00000018566 & Slc2a4 & & & -1.78 & \\
\hline ENSMUSG00000024978 & Gpam & & & -1.08 & -0.93 \\
\hline ENSMUSG00000040562 & Gstm2 & & & -1.48 & \\
\hline ENSMUSG00000031451 & Gas6 & & & -0.47 & \\
\hline ENSMUSG00000027984 & Hadh & & & -0.47 & \\
\hline ENSMUSG00000036594 & H2-Aa & & & -0.42 & \\
\hline ENSMUSG00000037025 & Foxa2 & & & & -0.63 \\
\hline
\end{tabular}




\begin{tabular}{|c|c|c|c|c|c|}
\hline Input IDs & Gene Symbol & CVvsC20 & CVvsC200 & HVvsH20 & HVvsH2OO \\
\hline ENSMUSG00000043013 & Onecut1 & & & & 0.96 \\
\hline ENSMUSG00000091971 & Hspa1a & & -3.95949 & & \\
\hline ENSMUSG00000041798 & Gck & & -0.98 & -1.01 & -0.63 \\
\hline ENSMUSG00000032115 & Hyou1 & & -1.35 & 0.52 & -0.75 \\
\hline ENSMUSG00000026185 & Igfbp5 & -1.14 & -1.39 & -0.97 & -0.54 \\
\hline ENSMUSG00000026072 & II1r1 & & & 0.58 & \\
\hline ENSMUSG00000027947 & II6ra & & & -0.67 & \\
\hline ENSMUSG00000038894 & Irs2 & & & 0.57 & 0.83 \\
\hline ENSMUSG00000020653 & Klf11 & 1.13 & 0.73 & & \\
\hline ENSMUSG00000052040 & Klf13 & & & -0.72 & \\
\hline ENSMUSG00000055148 & Klf2 & & -1.38 & -0.74 & \\
\hline ENSMUSG00000032796 & Lama1 & & & -0.63 & \\
\hline ENSMUSG00000020593 & Lpin1 & & -0.76 & -1.96 & -0.70 \\
\hline ENSMUSG00000024052 & Lpin2 & & & -0.73 & \\
\hline ENSMUSG00000015568 & Lpl & & 0.73 & 0.91 & \\
\hline ENSMUSG00000035202 & Lars2 & & & -1.38 & -1.58 \\
\hline ENSMUSG00000026688 & $\overline{\text { Mgst3 }}$ & & & -1.19 & \\
\hline ENSMUSG00000049723 & $\overline{\mathrm{Mmp12}}$ & & & -0.97 & \\
\hline ENSMUSG00000021025 & Nfkbia & & -0.51 & -0.46 & \\
\hline ENSMUSG00000032715 & $\overline{\text { Trib3 }}$ & 1.21 & 0.91 & -0.59 & \\
\hline ENSMUSG00000024413 & $\overline{\mathrm{Npc1}}$ & & 0.68 & & \\
\hline ENSMUSG00000030659 & Nucb2 & & & 0.82 & \\
\hline ENSMUSG00000023034 & $\overline{\mathrm{Nr4a1}}$ & & -0.63 & & 0.94 \\
\hline ENSMUSG00000096054 & $\overline{\text { Syne1 }}$ & 0.62 & & & \\
\hline ENSMUSG00000041827 & Oasl1 & & 1.15 & & \\
\hline ENSMUSG00000000168 & Dlat & & & -0.76 & \\
\hline ENSMUSG00000029304 & Spp1 & & & 0.48 & \\
\hline ENSMUSG00000028211 & Trp53inp1 & & & & 1.08 \\
\hline ENSMUSG00000044254 & Pcsk9 & & & -0.99 & -1.20 \\
\hline ENSMUSG00000028525 & $\underline{P d e 4 b}$ & & 0.73 & & \\
\hline ENSMUSG00000038508 & Gdf15 & & 1.52 & 1.22 & 1.08 \\
\hline ENSMUSG00000019577 & Pdk4 & & 1.04 & & 1.37 \\
\hline ENSMUSG00000025509 & Pnpla2 & & 0.85 & & \\
\hline ENSMUSG00000020893 & Per1 & & & -0.97 & \\
\hline ENSMUSG00000033871 & Ppargc1b & & 0.86 & & \\
\hline ENSMUSG00000032462 & Pik3cb & & 0.89 & & \\
\hline ENSMUSG00000041417 & Pik3r1 & & -0.47 & & \\
\hline ENSMUSG00000045038 & Prkce & & & -0.53 & \\
\hline ENSMUSG00000000440 & Pparg & & 0.62 & & \\
\hline ENSMUSG00000027540 & Ptpn1 & & & 0.61 & \\
\hline ENSMUSG00000028150 & Rorc & -0.74 & 0.88 & & 0.53 \\
\hline ENSMUSG00000015843 & Rxrg & & 0.83 & & \\
\hline ENSMUSG00000043895 & S1pr2 & & 1.20 & & \\
\hline ENSMUSG00000074115 & Saa1 & & -1.44 & 2.34 & 2.41 \\
\hline ENSMUSG00000041567 & Serpina12 & & -1.04 & 0.65 & \\
\hline ENSMUSG00000019970 & Sgk1 & & & & 0.77 \\
\hline ENSMUSG00000038351 & Sgsm2 & & 0.76 & & \\
\hline
\end{tabular}


Table 49 (continued)

Targets associated with metabolic diseases enrichments (Type 2 Diabetes Mellitus) and their change vs. vehicle control in each comparison

\begin{tabular}{|c|c|c|c|c|c|}
\hline Input IDs & $\begin{array}{l}\text { Gene } \\
\text { Symbol }\end{array}$ & CVvsC20 & CVvsC200 & HVvsH2O & HVvsH2OO \\
\hline ENSMUSG00000020027 & Socs2 & & & 1.10 & 0.71 \\
\hline ENSMUSG00000053113 & Socs3 & & & 1.08 & \\
\hline ENSMUSG00000025006 & Sorbs1 & & 0.64 & -0.50 & \\
\hline ENSMUSG00000037465 & Klf10 & 0.86 & & -0.54 & -1.29 \\
\hline ENSMUSG00000028599 & Tnfrsf1b & & & & 0.58 \\
\hline ENSMUSG00000022797 & Tfrc & & & -0.80 & \\
\hline ENSMUSG00000028128 & $\overline{\mathrm{F3}}$ & & 1.32 & 0.85 & \\
\hline ENSMUSG00000020123 & Avpr1a & 0.98 & -1.29 & 0.72 & \\
\hline ENSMUSG00000027962 & Vcam1 & & 0.63 & -0.85 & \\
\hline ENSMUSG00000024924 & Vldlr & & & -2.54 & \\
\hline ENSMUSG00000028978 & Nos3 & & -0.87 & & \\
\hline ENSMUSG00000041653 & Pnpla3 & & & -1.78 & -1.17 \\
\hline
\end{tabular}

Metabolic disease analysis for each comparison indicated gene sets associated with endpoints

including diabetes mellitus. Differentially regulated gene targets are indicated. For each

comparison, fold change vs. control is shown. 
Table 50

Targets associated with metabolic disease enrichments (metabolic syndrome) and their change vs. vehicle control in each comparison

\begin{tabular}{|c|c|c|c|c|c|}
\hline Input IDs & Gene Symbol & CVvsC20 & CVvsC200 & HVvsH20 & HVvsH200 \\
\hline \multicolumn{6}{|c|}{ Metabolic Disease Biomarkers: Metabolic Syndrome } \\
\hline ENSMUSG00000031278 & Acsl4 & & -0.47 & 1.05 & \\
\hline ENSMUSG00000024391 & Apom & & & 0.76 & \\
\hline ENSMUSG00000055116 & Arntl & 2.2 & 1.04 & & \\
\hline ENSMUSG00000045730 & Adrb2 & -1.04 & & -1.49 & \\
\hline ENSMUSG00000002944 & $\mathrm{Cd} 36$ & -0.75 & 0.74 & -0.89 & \\
\hline ENSMUSG00000029238 & Clock & 0.77 & & & \\
\hline ENSMUSG00000068742 & Cry2 & & 0.48 & & \\
\hline ENSMUSG00000072082 & $\underline{\mathrm{Ccnf}}$ & & & 0.46 & \\
\hline ENSMUSG00000019768 & Esr1 & & & 0.57 & 0.69 \\
\hline ENSMUSG00000010663 & Fads1 & & & & -0.64 \\
\hline ENSMUSG00000028001 & Fga & & & 1.06 & \\
\hline ENSMUSG00000033831 & $\mathrm{Fgb}$ & & & 1.02 & \\
\hline ENSMUSG00000033860 & Fgg & & & 0.96 & \\
\hline ENSMUSG00000020429 & Igfbp1 & & & -0.46 & 1.08 \\
\hline ENSMUSG00000020427 & Igfbp3 & & 0.44 & & \\
\hline ENSMUSG00000026185 & Igfbp5 & -1.14 & -1.39 & -0.97 & -0.54 \\
\hline ENSMUSG00000027947 & I16ra & & & -0.674195 & \\
\hline ENSMUSG00000020593 & Lpin1 & & -0.76 & -1.96 & -0.70 \\
\hline ENSMUSG00000015568 & Lpl & & 0.72 & 0.91 & \\
\hline ENSMUSG00000059436 & Max & & -0.55 & & \\
\hline ENSMUSG00000032715 & Trib3 & 1.21 & 0.91 & -0.59 & \\
\hline ENSMUSG00000027820 & Mme & & 0.73 & -0.81 & \\
\hline ENSMUSG00000019577 & Pdk4 & & 1.04 & & 1.37 \\
\hline ENSMUSG00000055866 & Per2 & -1.39 & -0.74 & & \\
\hline ENSMUSG00000042632 & Pla2g6 & & 0.98 & & \\
\hline ENSMUSG00000023913 & Pla2g7 & & 0.65 & & \\
\hline ENSMUSG00000000440 & Pparg & & 0.62 & & \\
\hline ENSMUSG00000027540 & Ptpn1 & & & 0.61 & \\
\hline ENSMUSG00000027368 & $\overline{\text { Dusp2 }}$ & & -1.27 & & \\
\hline ENSMUSG00000020580 & Rock2 & & & -0.42 & \\
\hline ENSMUSG00000074115 & $\overline{\text { Saa1 }}$ & & -1.44 & 2.34 & 2.41 \\
\hline ENSMUSG00000041567 & Serpina12 & & -1.04 & 0.65 & \\
\hline ENSMUSG00000012428 & Steap4 & & -0.62 & 1.22 & \\
\hline ENSMUSG00000028978 & Nos3 & & -0.87 & & \\
\hline ENSMUSG00000041653 & Pnpla3 & & & -1.78 & -1.17 \\
\hline ENSMUSG00000027962 & Vcam1 & & 0.63 & -0.85 & \\
\hline
\end{tabular}


Table 50 (continued)

Targets associated with metabolic disease enrichments (metabolic syndrome) and their change vs. vehicle control in each comparison

\begin{tabular}{|c|c|c|c|c|c|}
\hline Input IDs & Gene Symbol & CVvsC20 & CVvsC200 & HVvsH20 & HVvsH200 \\
\hline ENSMUSG00000048376 & $\mathrm{F} 2 \mathrm{r}$ & & & 0.44 & \\
\hline ENSMUSG00000030748 & I14ra & & 0.57 & & \\
\hline ENSMUSG00000021871 & Pnp & & & 0.61 & \\
\hline ENSMUSG00000033220 & Rac2 & & 0.56 & & \\
\hline ENSMUSG00000057465 & $\underline{\text { Saa2 }}$ & & -1.37 & 2.06 & 2.62 \\
\hline ENSMUSG00000028599 & $\underline{\text { Tnfrsf1b }}$ & & & & 0.58 \\
\hline \multicolumn{6}{|c|}{ Metabolic disease analysis for each comparison indicated gene sets associated with endpoir } \\
\hline
\end{tabular}


Table 51

Targets associated with metabolic disease enrichments (insulin resistance) and their change vs. vehicle control in each comparison

\begin{tabular}{|c|c|c|c|c|c|}
\hline Input IDs & $\begin{array}{l}\text { Gene } \\
\text { Symbol }\end{array}$ & CVvsC20 & CVvsC200 & HVvsH20 & HVvsH2OO \\
\hline \multicolumn{6}{|c|}{ Metabolic Disease Biomarkers: Insulin resistance } \\
\hline ENSMUSG00000031278 & Acsl4 & & -0.47 & 1.05 & \\
\hline ENSMUSG00000035783 & Acta2 & & -0.96 & & -0.71 \\
\hline ENSMUSG00000024391 & Apom & & & 0.76 & \\
\hline ENSMUSG00000027792 & Bche & & & -0.67 & \\
\hline ENSMUSG00000055116 & Arntl & & 1.04 & & \\
\hline ENSMUSG00000045730 & Adrb2 & & & & \\
\hline ENSMUSG00000036098 & Myrf & & & 0.51 & \\
\hline ENSMUSG00000035042 & $\mathrm{Ccl} 5$ & & & -0.97 & \\
\hline ENSMUSG00000002944 & $\mathrm{Cd} 36$ & -0.75 & 0.74 & -0.89 & \\
\hline ENSMUSG00000029238 & Clock & 0.77 & & & \\
\hline ENSMUSG00000068742 & Cry2 & & 0.48 & & \\
\hline ENSMUSG00000072082 & $\underline{\text { Conf }}$ & & & 0.46 & \\
\hline ENSMUSG00000041220 & Elovi6 & & & -0.89 & -0.53 \\
\hline ENSMUSG00000019768 & Esr1 & & & 0.57 & 0.69 \\
\hline ENSMUSG00000010663 & Fads1 & & & & -0.64 \\
\hline ENSMUSG00000024664 & Fads3 & -0.91 & & -0.56 & -0.91 \\
\hline ENSMUSG00000044167 & $\overline{\text { Foxo1 }}$ & & & -0.53 & \\
\hline ENSMUSG00000048756 & $\overline{\text { Foxo3 }}$ & & & -0.79 & \\
\hline ENSMUSG00000028001 & Fga & & & 1.06 & \\
\hline ENSMUSG00000033831 & $\mathrm{Fgb}$ & & & 1.02 & \\
\hline ENSMUSG00000033860 & Fgg & & & 0.96 & \\
\hline ENSMUSG00000049721 & Gal3st1 & & & & \\
\hline ENSMUSG00000029992 & Gfpt1 & & -0.75 & & -0.56 \\
\hline ENSMUSG00000018566 & Slc2a4 & & & & \\
\hline ENSMUSG00000021670 & $\mathrm{Hmgcr}$ & & & -0.73 & \\
\hline ENSMUSG00000043013 & Onecut1 & & & & 0.96 \\
\hline ENSMUSG00000041798 & Gck & & -0.98 & & -0.63 \\
\hline ENSMUSG00000020429 & Igfbp1 & & & -0.46 & 1.08 \\
\hline ENSMUSG00000020427 & Igfbp3 & & 0.44 & & \\
\hline ENSMUSG00000026185 & Igfbp5 & & & -0.97 & -0.54 \\
\hline ENSMUSG00000027947 & $\underline{116 \mathrm{ra}}$ & & & -0.67 & \\
\hline ENSMUSG00000038894 & $\operatorname{Irs2}$ & & & 0.57 & 0.83 \\
\hline ENSMUSG00000020593 & Lpin1 & & -0.76 & & -0.70 \\
\hline ENSMUSG00000024052 & Lpin2 & & & -0.73 & \\
\hline ENSMUSG00000015568 & $\underline{\text { Lpl }}$ & & 0.72 & 0.91 & \\
\hline ENSMUSG00000054612 & Mgmt & & & & \\
\hline ENSMUSG00000059436 & Max & & -0.55 & & \\
\hline ENSMUSG00000054764 & Mtnr1a & & & & \\
\hline ENSMUSG00000021025 & Nfkbia & & -0.51 & -0.46 & \\
\hline
\end{tabular}


Table 51 (continued)

Targets associated with metabolic disease enrichments (insulin resistance) and their change vs. vehicle control in each comparison

\begin{tabular}{|c|c|c|c|c|c|}
\hline Input IDs & Gene Symbol & CVvsC20 & CVvsC200 & HVvsH2O & HVvsH2OO \\
\hline ENSMUSG00000032715 & Trib3 & 1.21 & 0.91 & -0.59 & \\
\hline ENSMUSG00000027820 & Mme & & 0.73 & -0.81 & \\
\hline ENSMUSG00000019577 & Pdk4 & & 1.04 & & \\
\hline ENSMUSG00000025509 & Pnpla2 & & 0.85 & & \\
\hline ENSMUSG00000055866 & Per2 & & -0.74 & & \\
\hline ENSMUSG00000032462 & Pik3cb & & 0.89 & & \\
\hline ENSMUSG00000041417 & Pik3r1 & & -0.47 & & \\
\hline ENSMUSG00000042632 & Pla2g6 & & 0.98 & & \\
\hline ENSMUSG00000023913 & Pla2g7 & & 0.65 & & \\
\hline ENSMUSG00000000440 & Pparg & & 0.62 & & \\
\hline ENSMUSG00000027540 & Ptpn1 & & & 0.61 & \\
\hline ENSMUSG00000027368 & Dusp2 & & & & \\
\hline ENSMUSG00000020580 & Rock2 & & & -0.42 & \\
\hline ENSMUSG00000074115 & $\underline{\text { Saa1 }}$ & & & & \\
\hline ENSMUSG00000075701 & Selenos & & -0.47 & & \\
\hline ENSMUSG00000041567 & Serpina12 & & & 0.65 & \\
\hline ENSMUSG00000060807 & Serpina6 & -0.97 & & & \\
\hline ENSMUSG00000053113 & Socs3 & & & 1.08 & \\
\hline ENSMUSG00000012428 & Steap4 & & -0.62 & 1.22 & \\
\hline ENSMUSG00000028599 & Tnfrsf1b & & & & 0.58 \\
\hline ENSMUSG00000027962 & Vcam1 & & 0.63 & -0.85 & \\
\hline ENSMUSG00000044786 & Zfp36 & & -0.53 & & \\
\hline ENSMUSG00000028978 & Nos3 & & -0.87 & & \\
\hline ENSMUSG00000041653 & Pnpla3 & & & & \\
\hline ENSMUSG00000008845 & Cd163 & 0.84 & & & \\
\hline ENSMUSG00000048376 & F2r & & & 0.44 & \\
\hline ENSMUSG00000044674 & $\underline{\text { Fzd1 }}$ & & 0.53 & & \\
\hline ENSMUSG00000030748 & $\| 4 \mathrm{ra}$ & & 0.57 & & \\
\hline ENSMUSG00000021871 & Pnp & & & 0.61 & \\
\hline ENSMUSG00000033871 & Ppargc1b & & 0.86 & & \\
\hline ENSMUSG00000033220 & Rac2 & & 0.56 & & \\
\hline ENSMUSG00000015843 & $\overline{\text { Rxrg }}$ & & 0.83 & & \\
\hline ENSMUSG00000057465 & Saa2 & & & & \\
\hline $\begin{array}{l}\text { Metabolic disease analys } \\
\text { including insulin resistanc } \\
\text { comparison, fold change }\end{array}$ & ferentially regul & indicated $\mathrm{g}$ & ene sets as & ociated wit & $\begin{array}{l}\text { e endpoints } \\
\text { each }\end{array}$ \\
\hline
\end{tabular}


proportion of the DTG sets. Comparison of these results to outputs of the same network frame showing DTG sets in vitro primary hepatocyte exposure to prototypical ligands for these receptors, however, indicates that the sets of direct transcriptional targets as curated by MetaCore $^{\mathrm{TM}}$ are probably not comprehensive (data not shown). Other software tools may be needed to further explore these relationships.

Analysis of dose-within-diet comparisons indicated enrichment in disease-associated transcription factors

Transcription factor analysis for the CVvsC20, CVvsC200, $\mathrm{HVvsH} 20$ and $\mathrm{HVvsH} 200$ are shown in Tables 52-55. These analyses are based on gene ontology, therefore the transcription factors ranked highest in the list of engaged transcription factors were those with the most literature-based relationships among the DTGs in each dataset. Many of these connections are based on chromatin immunoprecipitation studies or computational binding site prediction, and are of unspecified effect, however, when only the defined relationships (activation or inhibition via transcriptional regulation) were considered, we were able to develop a percent activation for each listed transcription factor under each condition: the number differentially regulated in a direction consistent with TF activation/total differentially regulated. These are listed in Tables 56 and 57. Overall, transcription factors tended to increase in percent activation with increasing PCB concentration and decrease with HFD exposure. Interestingly, transcription factors which were targets of PXR regulation by binding showed evidence of increased activation at the $20 \mathrm{mg} / \mathrm{kg}$ exposure, compared to the $200 \mathrm{mg} / \mathrm{kg}$ exposure, shown in Figure 13. PXR targets of regulation by protein-protein interaction.

Specific targets within pathways engaged in these experiments are related to metabolic syndrome components, including fatty liver disease and diabetes, as well as liver damage and regeneration pathways.

Several of the targets differentially regulated under one or more of the experimental conditions have been characterized as potential contributors to metabolic diseases, including 


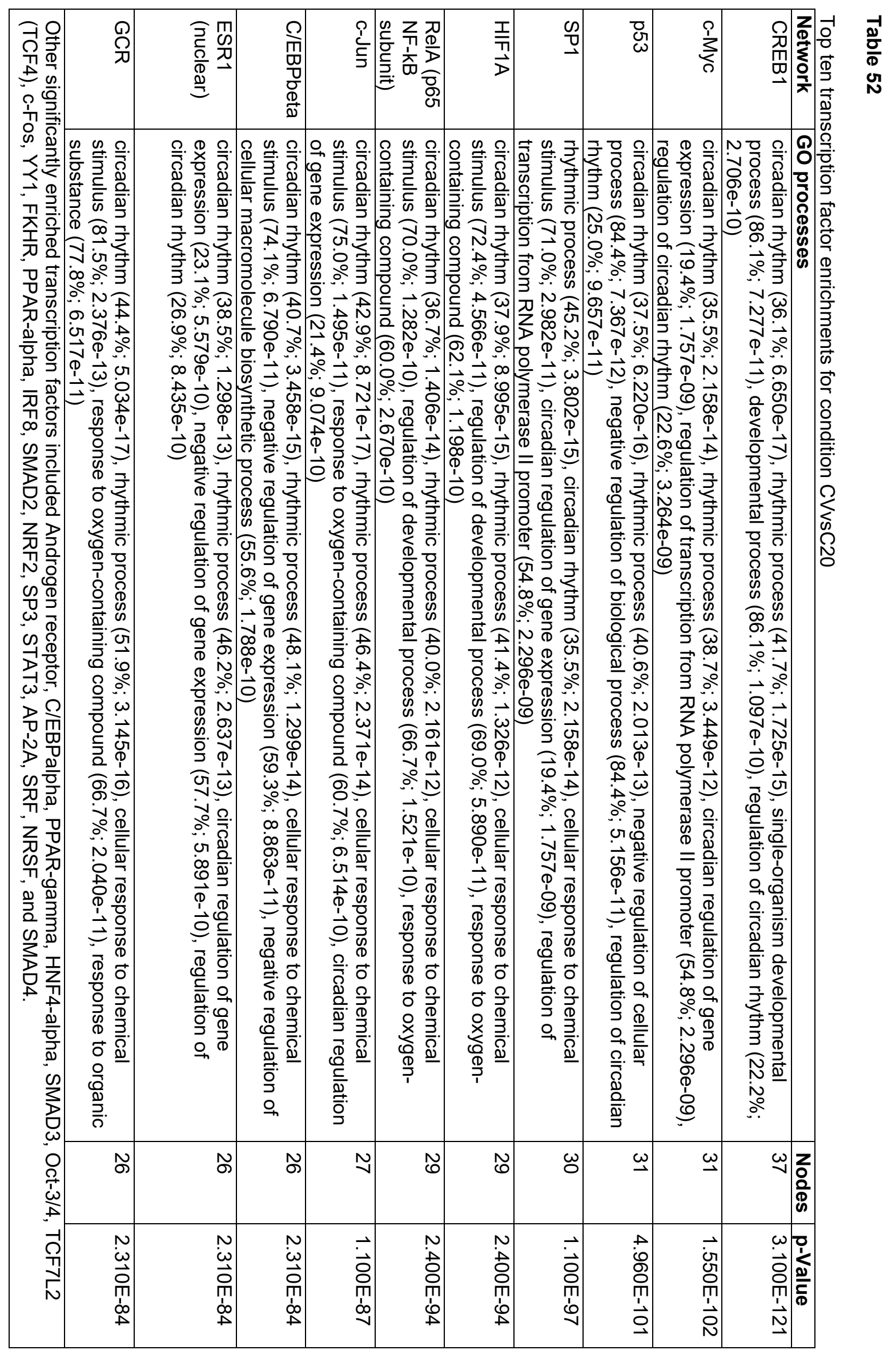




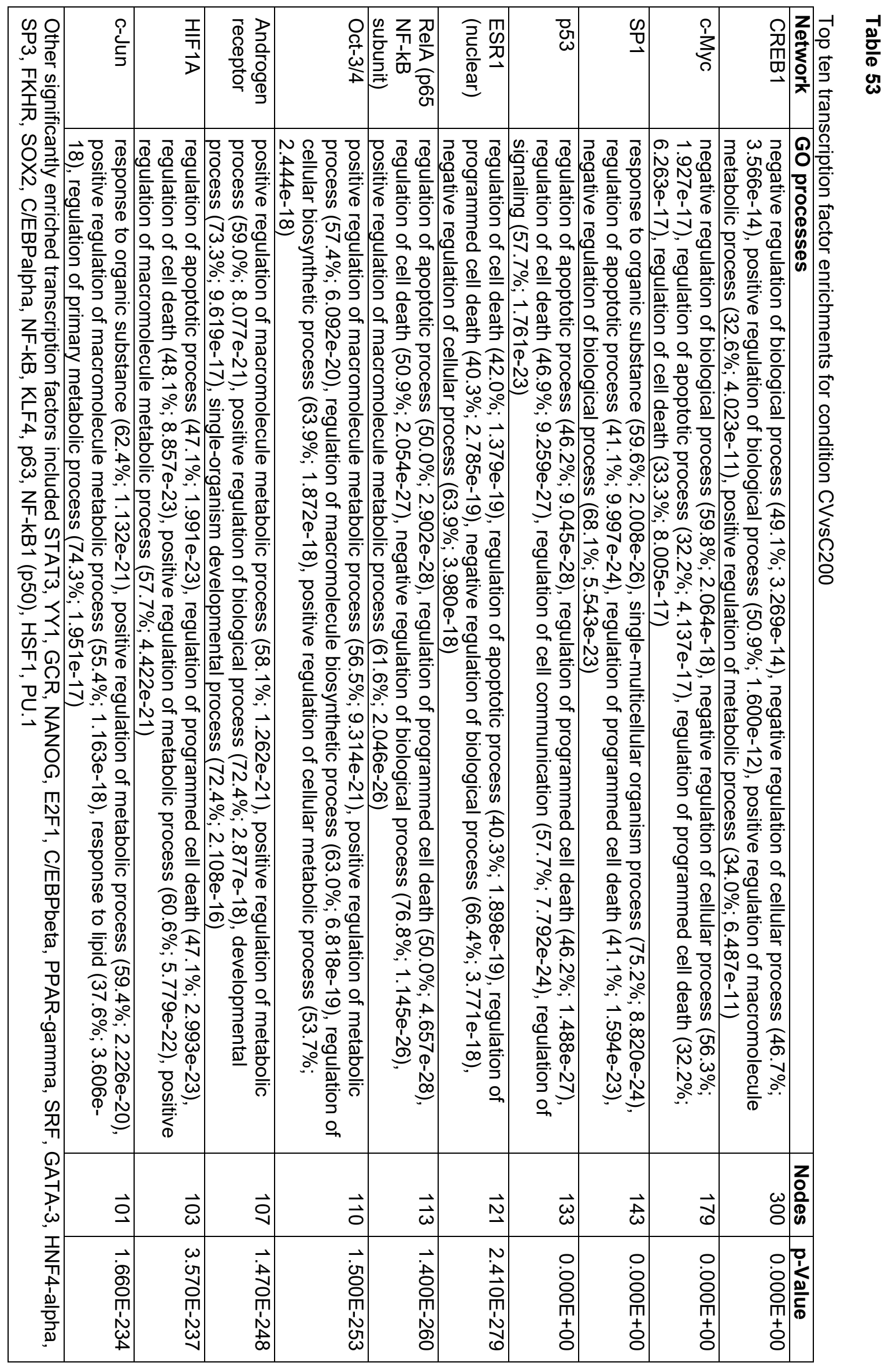




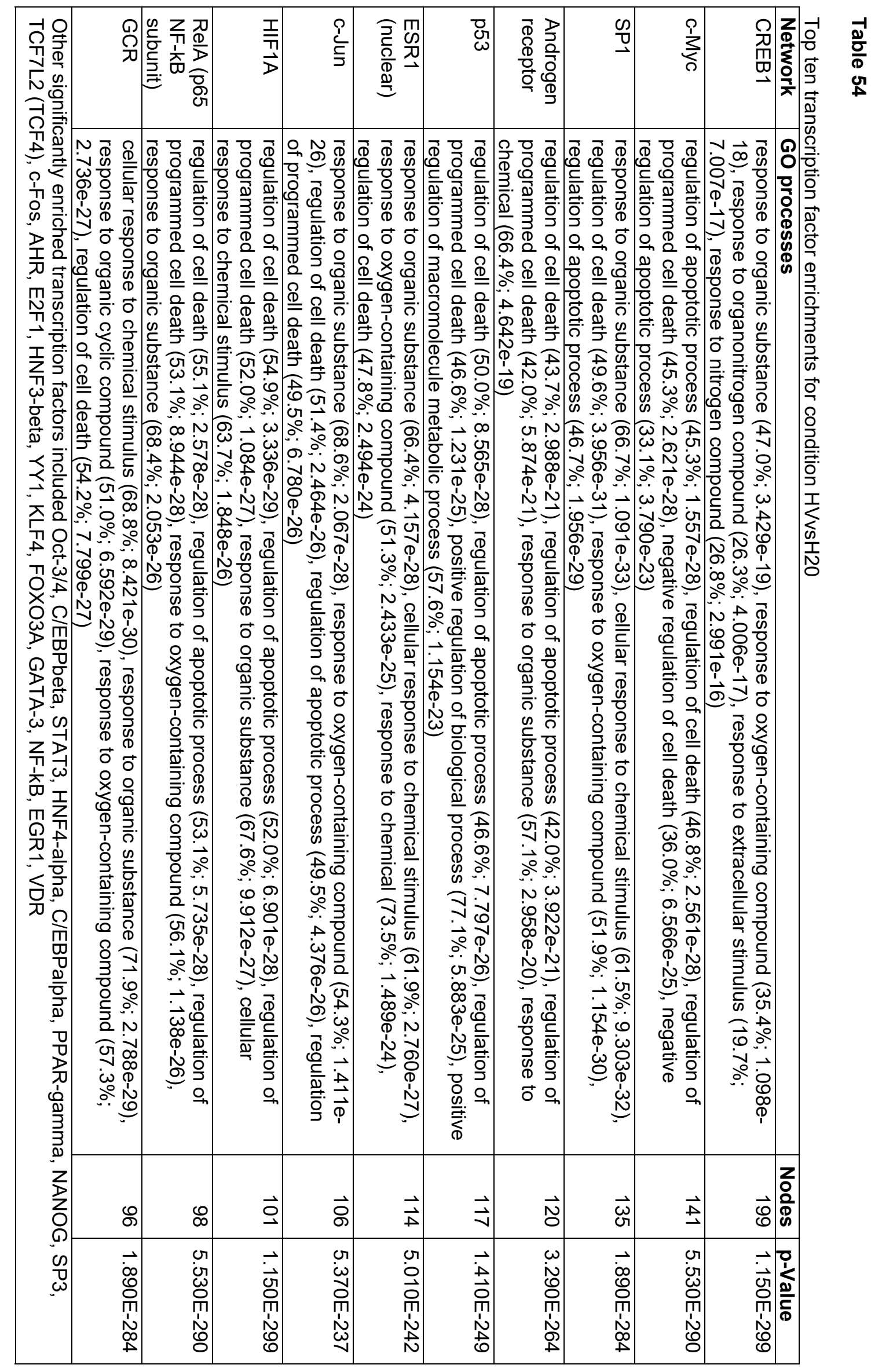




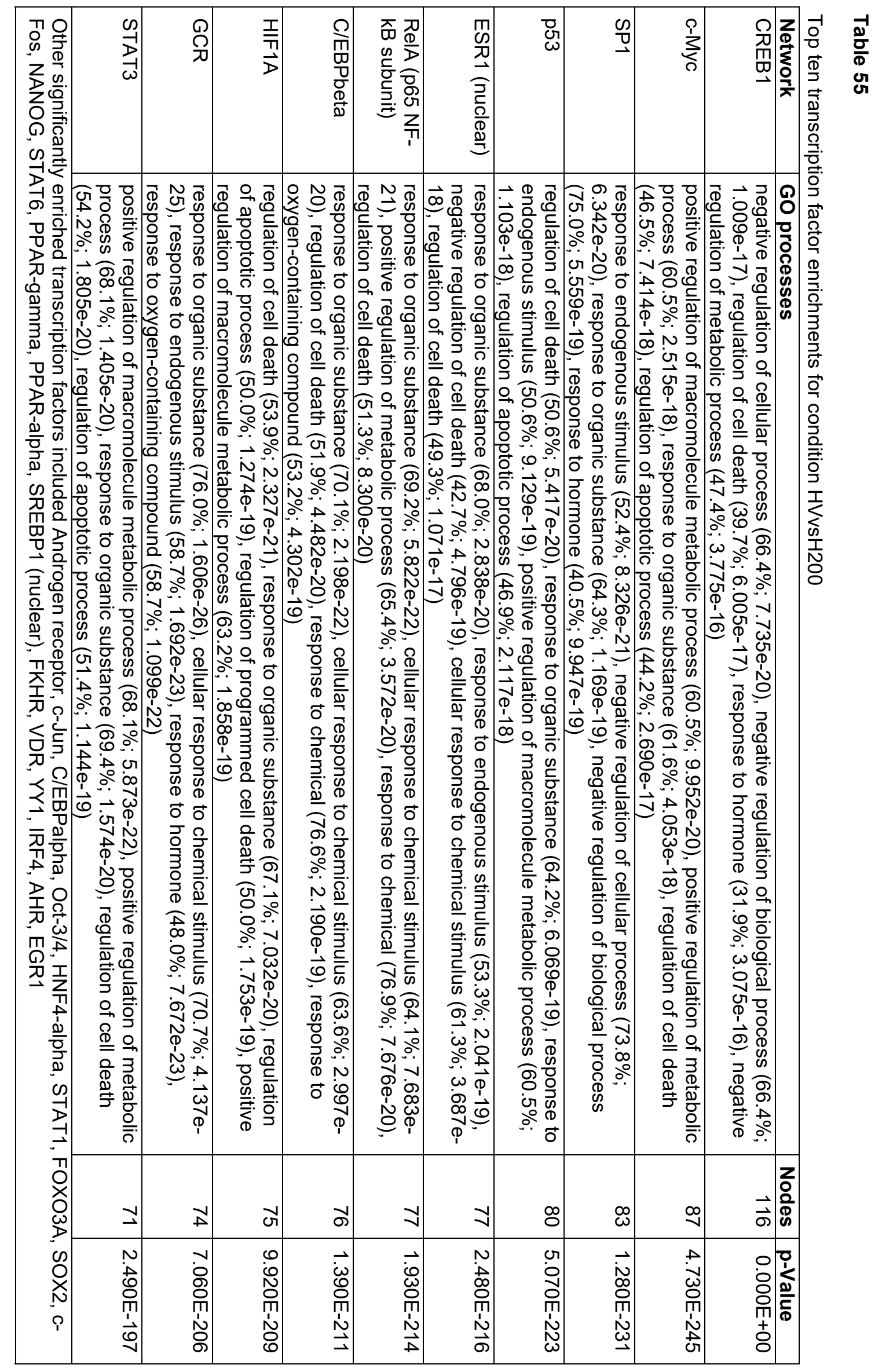


Table 56

Percent activation for engaged transcription factors identified in all conditions

\begin{tabular}{|l|c|c|c|c|c|c|c|c|}
\hline & \multicolumn{2}{|c|}{ CVvsC20 } & \multicolumn{2}{c|}{ CVvsC200 } & \multicolumn{2}{c|}{ HVvsH20 } & \multicolumn{2}{c|}{ HVvsH200 } \\
\hline & Total & $\begin{array}{c}\% \\
\text { CWA }\end{array}$ & Total & $\begin{array}{c}\% \\
\text { CWA }\end{array}$ & Total & $\begin{array}{c}\% \\
\text { CWA }\end{array}$ & Total & $\begin{array}{c}\% \\
\text { CWA }\end{array}$ \\
\hline CREB1 & 31 & $16 \%$ & 48 & $52 \%$ & 168 & $13 \%$ & 93 & $24 \%$ \\
\hline c-Myc & 25 & $32 \%$ & 72 & $32 \%$ & 116 & $28 \%$ & 62 & $18 \%$ \\
\hline p53 & 19 & $37 \%$ & 68 & $60 \%$ & 92 & $34 \%$ & 50 & $46 \%$ \\
\hline SP1 & 23 & $30 \%$ & 69 & $59 \%$ & 109 & $32 \%$ & 58 & $43 \%$ \\
\hline HIF1A & 21 & $38 \%$ & 54 & $63 \%$ & 62 & $34 \%$ & 44 & $48 \%$ \\
\hline $\begin{array}{l}\text { RelA (p65 NF- } \\
\text { kB subunit) }\end{array}$ & 16 & $25 \%$ & 53 & $64 \%$ & 69 & $38 \%$ & 43 & $56 \%$ \\
\hline c-Jun & 14 & $50 \%$ & 37 & $73 \%$ & 71 & $38 \%$ & 35 & $49 \%$ \\
\hline C/EBPbeta & 14 & $64 \%$ & 34 & $56 \%$ & 58 & $47 \%$ & 41 & $61 \%$ \\
\hline ESR1 (nuclear) & 18 & $33 \%$ & 52 & $54 \%$ & 84 & $33 \%$ & 50 & $50 \%$ \\
\hline GCR & 16 & $56 \%$ & 47 & $43 \%$ & 60 & $27 \%$ & 37 & $68 \%$ \\
\hline $\begin{array}{l}\text { Androgen } \\
\text { receptor }\end{array}$ & 13 & $15 \%$ & 37 & $54 \%$ & 89 & $25 \%$ & 33 & $24 \%$ \\
\hline C/EBPalpha & 13 & $46 \%$ & 25 & $60 \%$ & 47 & $38 \%$ & 33 & $39 \%$ \\
\hline PPAR-gamma & 9 & $78 \%$ & 30 & $57 \%$ & 36 & $28 \%$ & 15 & $47 \%$ \\
\hline HNF4-alpha & 14 & $50 \%$ & 29 & $62 \%$ & 45 & $33 \%$ & 30 & $53 \%$ \\
\hline Oct-3/4 & 12 & $42 \%$ & 54 & $50 \%$ & 56 & $39 \%$ & 31 & $39 \%$ \\
\hline YY1 & 7 & $29 \%$ & 19 & $42 \%$ & 33 & $15 \%$ & 15 & $27 \%$ \\
\hline FKHR & 7 & $43 \%$ & 24 & $63 \%$ & 24 & $21 \%$ & 18 & $50 \%$ \\
\hline STAT3 & 10 & $50 \%$ & 44 & $45 \%$ & 62 & $48 \%$ & 37 & $57 \%$ \\
\hline
\end{tabular}

Relative activation of the most enriched transcription factors (second column for each

comparison) was determined by comparing the proportion of differentially transcribed targets in

which the direction of transcription was concordant with activation of the transcription factor, based on the MetaCore curated TF $\rightarrow$ target relationship and normalized to the total number of targets for that TF differentially transcribed in our experiment (first column for each comparison).

This table shows \% engagement for TFs which were enriched in all group 1-2 comparisons. 
Table 57

Percent activation for engaged transcription factors identified in 1-3 conditions

\begin{tabular}{|c|c|c|c|c|c|c|c|c|}
\hline & \multicolumn{2}{|c|}{ CVvsC20 } & \multicolumn{2}{|c|}{ CVvsC200 } & \multicolumn{2}{|c|}{ HVvsH2O } & \multicolumn{2}{|c|}{ HVvsH2OO } \\
\hline & Total & $\begin{array}{c}\% \\
C W A\end{array}$ & Total & $\begin{array}{c}\% \\
C W A\end{array}$ & Total & $\begin{array}{c}\% \\
C W A\end{array}$ & Total & $\begin{array}{c}\% \\
C W A\end{array}$ \\
\hline SP3 & 6 & $33 \%$ & 25 & $52 \%$ & 38 & $37 \%$ & 0 & - \\
\hline TCF7L2 (TCF4) & 7 & $43 \%$ & 0 & - & 41 & $34 \%$ & 0 & - \\
\hline c-FOS & 9 & $33 \%$ & 0 & - & 35 & $49 \%$ & 22 & $55 \%$ \\
\hline SMAD3 & 8 & $38 \%$ & 0 & - & 0 & - & 0 & - \\
\hline PPAR-alpha & 8 & $50 \%$ & 0 & - & - & - & 14 & $43 \%$ \\
\hline IRF8 & 8 & $13 \%$ & 0 & - & - & - & 0 & - \\
\hline SMAD2 & 5 & $20 \%$ & 0 & - & - & - & - & - \\
\hline NRF2 & 6 & $83 \%$ & 0 & - & - & - & - & - \\
\hline$A P-2 A$ & 8 & $25 \%$ & 0 & - & - & - & - & - \\
\hline SRF & 5 & $40 \%$ & 26 & $42 \%$ & 0 & - & - & - \\
\hline NRSF & 4 & $25 \%$ & 0 & - & - & - & - & - \\
\hline SMAD4 & 5 & $100 \%$ & 0 & - & - & - & - & - \\
\hline NANOG & 0 & - & 27 & $48 \%$ & 45 & $31 \%$ & 23 & $26 \%$ \\
\hline$E 2 F 1$ & 0 & - & 21 & $81 \%$ & 37 & $35 \%$ & 0 & - \\
\hline GATA-3 & 0 & - & 9 & $33 \%$ & 26 & $15 \%$ & 0 & - \\
\hline SOX2 & 0 & - & 18 & $39 \%$ & 0 & - & 24 & $33 \%$ \\
\hline$N F-k B$ & 0 & - & 27 & $70 \%$ & 28 & $32 \%$ & 0 & - \\
\hline KLF4 & 0 & - & 20 & $55 \%$ & 29 & $62 \%$ & 0 & - \\
\hline p63 & 0 & - & 25 & $52 \%$ & 0 & - & - & - \\
\hline$N F-k B 1(p 50)$ & 0 & - & 13 & $62 \%$ & 0 & - & - & - \\
\hline HSF1 & 0 & - & 27 & $26 \%$ & 0 & - & - & - \\
\hline PU.1 & 0 & - & 26 & $65 \%$ & 0 & - & - & - \\
\hline AHR & 0 & - & 0 & - & 33 & $12 \%$ & 18 & $22 \%$ \\
\hline HNF3-beta & 0 & - & 0 & - & 26 & $15 \%$ & 19 & $47 \%$ \\
\hline FOXОЗА & 0 & - & 0 & - & 29 & $28 \%$ & 23 & $57 \%$ \\
\hline EGR1 & 10 & $50 \%$ & 0 & - & 32 & $47 \%$ & 21 & $43 \%$ \\
\hline VDR & 0 & - & 0 & - & 26 & $27 \%$ & 16 & $50 \%$ \\
\hline STAT1 & 0 & - & 0 & - & 0 & - & 20 & $70 \%$ \\
\hline STAT6 & 0 & - & 0 & - & 0 & - & 16 & $0 \%$ \\
\hline SREBP1 (nuclear) & 0 & - & 0 & - & 0 & - & 14 & $7 \%$ \\
\hline IRF4 & 0 & - & 0 & - & 0 & - & 16 & $38 \%$ \\
\hline
\end{tabular}

Relative activation of the most enriched transcription factors (second column for each

comparison) was determined by comparing the proportion of differentially transcribed targets in which the direction of transcription was concordant with activation of the transcription factor, based on the MetaCore curated TF $\rightarrow$ target relationship and normalized to the total number of targets for that TF differentially transcribed in our experiment (first column for each comparison).

This table shows \% engagement for TFs which were enriched in 1, 2, or 3 of the group 1-2 comparisons. 


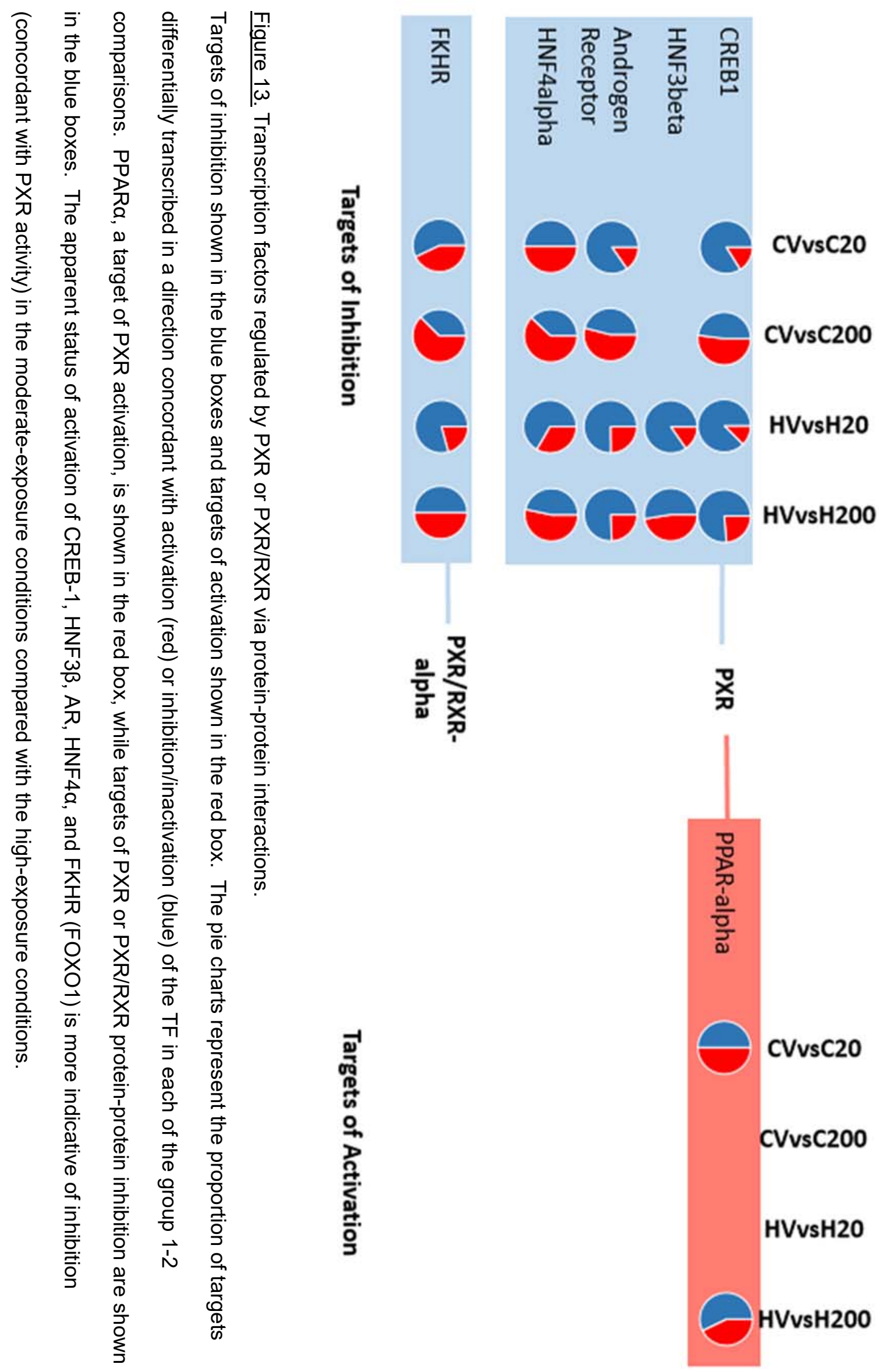


Trib3/NIPK, Arntl, and claudin-1. Associated pathway maps are shown in Figures 14-16, respectively.

The Tribbles 3 pseudokinase (NIPK or TRIB3) interrupts insulin signaling by binding and preventing phosphorylation of AKT, thereby promoting gluconeogenic down-stream targets such as PEPCK and diminishing the activity of GSK3B and associated glycogen synthesis ${ }^{127,}, 128$ (Figure 14). TRIB3 can also dephosphorylate several MAP kinases and AMPK. TRIB3 is activated by PPAR $\alpha$ and by endoplasmic reticulum stress (PERK-elF2alpha-ATF4-CHOP arm of the unfolded protein response), ${ }^{129}$ thereby providing a mechanism by which ER stress can modulate the transduction energy and growth factor signaling to intermediary metabolic and developmental transcriptional outputs. The human TRIB3 polymorphism Q84R, which enhances its inhibition of $\mathrm{AKT}$, has been linked to increased prevalence of insulin resistance and associated cardiovascular risk ${ }^{130}$. TRIB3 is up-regulated in obesity and metabolic syndrome.

The arylhydrocarbon receptor nuclear translocator-like (Arntl) forms a heterodimer with CLOCK, an active complex driving transcription of circadian output genes such as the Period (Per1, 2, etc.) and Cryptochrome (Cry1, 2, etc.) genes (Figure 15). Another target up-regulated in all four comparisons was nuclear factor interleukin-3 (Nfil-3, or E4BP4), which encodes a protein that represses expression of the Period genes ${ }^{131,132}$. Processes related to circadian rhythm and control of rhythmic processes were enriched with moderate PCB exposure in our model. Again, disruption of normal circadian rhythm may be both cause and effect in metabolic disease ${ }^{133,134}$. The oscillatory patterns of circadian clock output genes (BMAL/CLOCK target genes) are disrupted in response to nutritional challenges ${ }^{135,136}$. Disrupted sleep patterns due to sleep apnea $^{137}$ or shift work ${ }^{138}$ are also associated with metabolic disease progression. Another target of circadian rhythm circuitry is Kruppel-like factor 9 (KLF9), a transcription factor which expressed in epithelial cells and regulates daytime-specific expression of differentiation-related targets (EII3) as well as targets related to glucocorticoid signaling (Fkbp5) $)^{139}$.

Other targets associated with cell differentiation/identity, epithelial-to-mesenchymal transition and regenerative processes were differentially regulated, particularly within the moderate exposure models. These included up-regulated cell adhesion proteins such as claudin- 


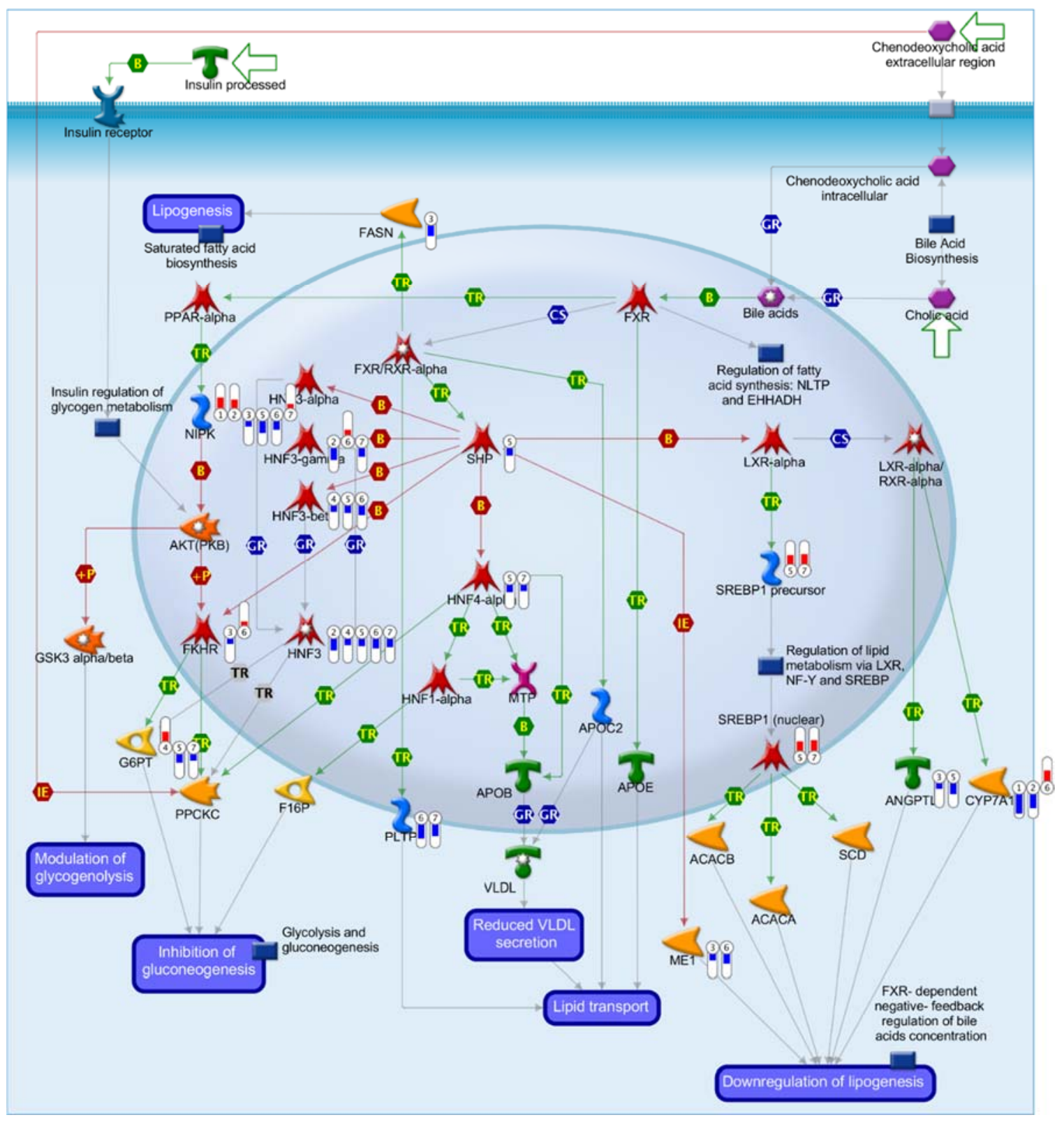

Figure 14. Nipk/Trib3 signaling pathway downstream of PPAR-alpha.

PCB exposure and PCB/HFD coexposures induced transcriptional changes in elements of signaling pathways downstream of PPAR-alpha. In particular, the tribbles pseudokinase (Nipk/Trib3) differentially regulated in all comparisons: CVvsC20 (1), CVvsC200 (2), HVvsH2O (3), HVvsH200 (4), C20vsH20 (5), C200vsH200 (6), and CVvsHV (7). Notably, transcription was upregulated in both control diet comparisons and in CVvsHV and downregulated in other comparisons. Image generated using MetaCore ${ }^{\mathrm{TM}}$ from Thomson Reuters. 


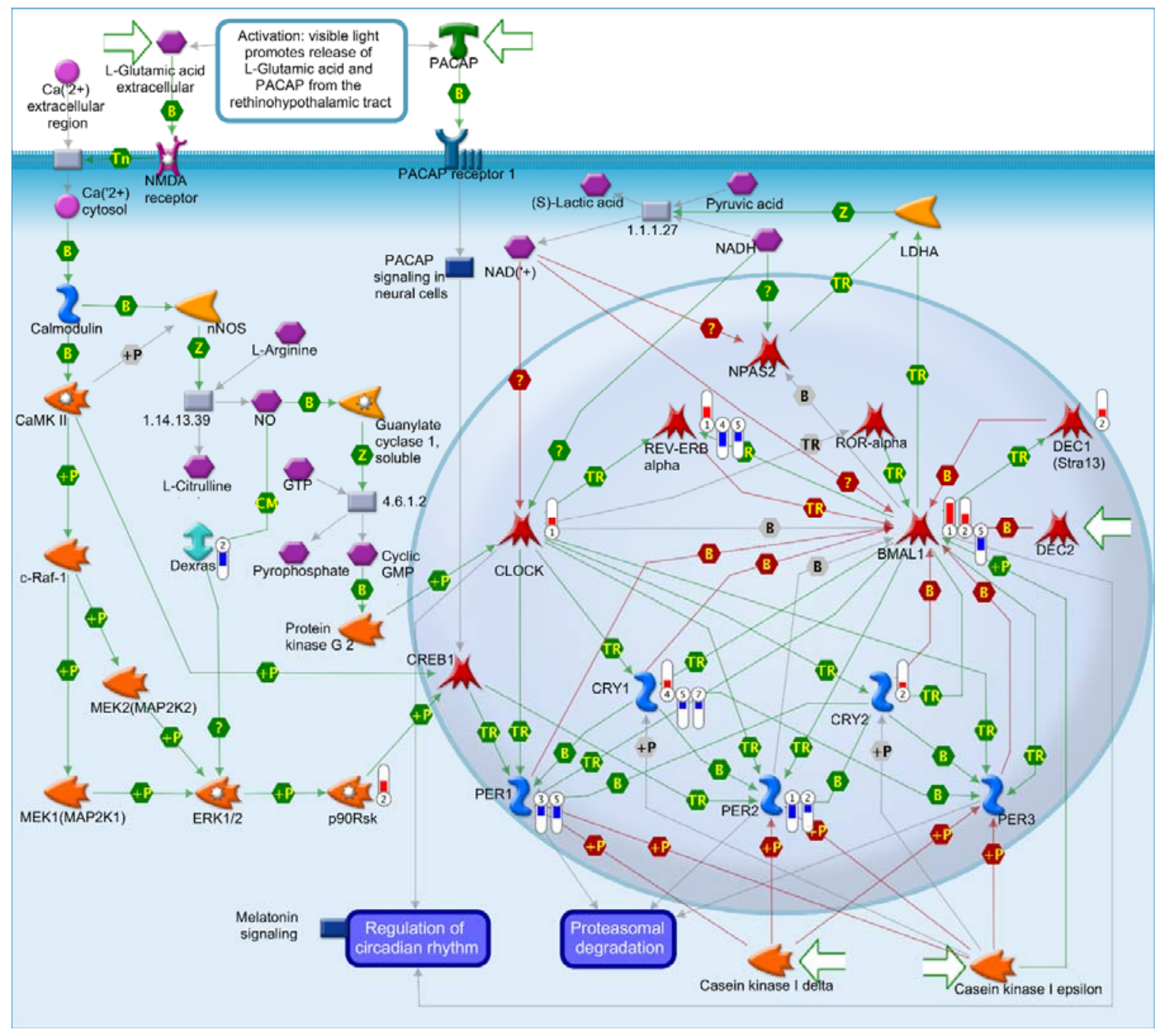

Figure 15. Arntl/Bmal1 pathway related to circadian signaling.

PCB exposure and PCB/HFD coexposures induced transcriptional changes in elements of signaling pathways related to circadian signaling. In particular, the arylhydrocarbon receptor nuclear translocator-like (Arntl/Bmal1) was differentially regulated in comparison CVvsC20 (1), CVvsC200 (2), and $\mathrm{C} 20$ vsH20 (5). Clock output targets such as Per1/2, and Cry1/2 were differentially regulated in comparisons $\mathrm{HVvsH} 20$ (3), $\mathrm{HVvsH} 200$ (4), and $\mathrm{CVvsHV}(7)$, as well as comparison 5. Image generated using MetaCore ${ }^{\mathrm{TM}}$ from Thomson Reuters. 
1, endothelin-1, and vimentin, changes in which signal epithelial-to-mesenchymal or mesenchymal-to-epithelial transitions in hepatocytes ${ }^{140}$ (Figure 16). Other differentially regulated targets in this category, including Frizzled, bone morphogenic protein 2 (BMP-2, a TGF $\beta$-family member), Kruppel-like factors 10/11 (KLF-10, KLF-11: TGF $\beta$-inducible growth regulators) and ID$1 / 2$ (inhibitor of DNA binding) are components of multiple development and differentiation pathways such as WNT (Frizzled), Hedgehog (BMP2), TGFß (BMP2, TIEG), and Hippo (ID-1/2). Alterations in cell identity that accompany EMT are classically associated with neoplastic liver disease $^{141}$, however there is evidence to link alterations in Hedgehog ${ }^{142,143}$, TGF $\beta^{144}$, WNT ${ }^{145,}$ 146, and Hippo ${ }^{147}$ signaling to the progression of steatohepatitis and fibrosis. 


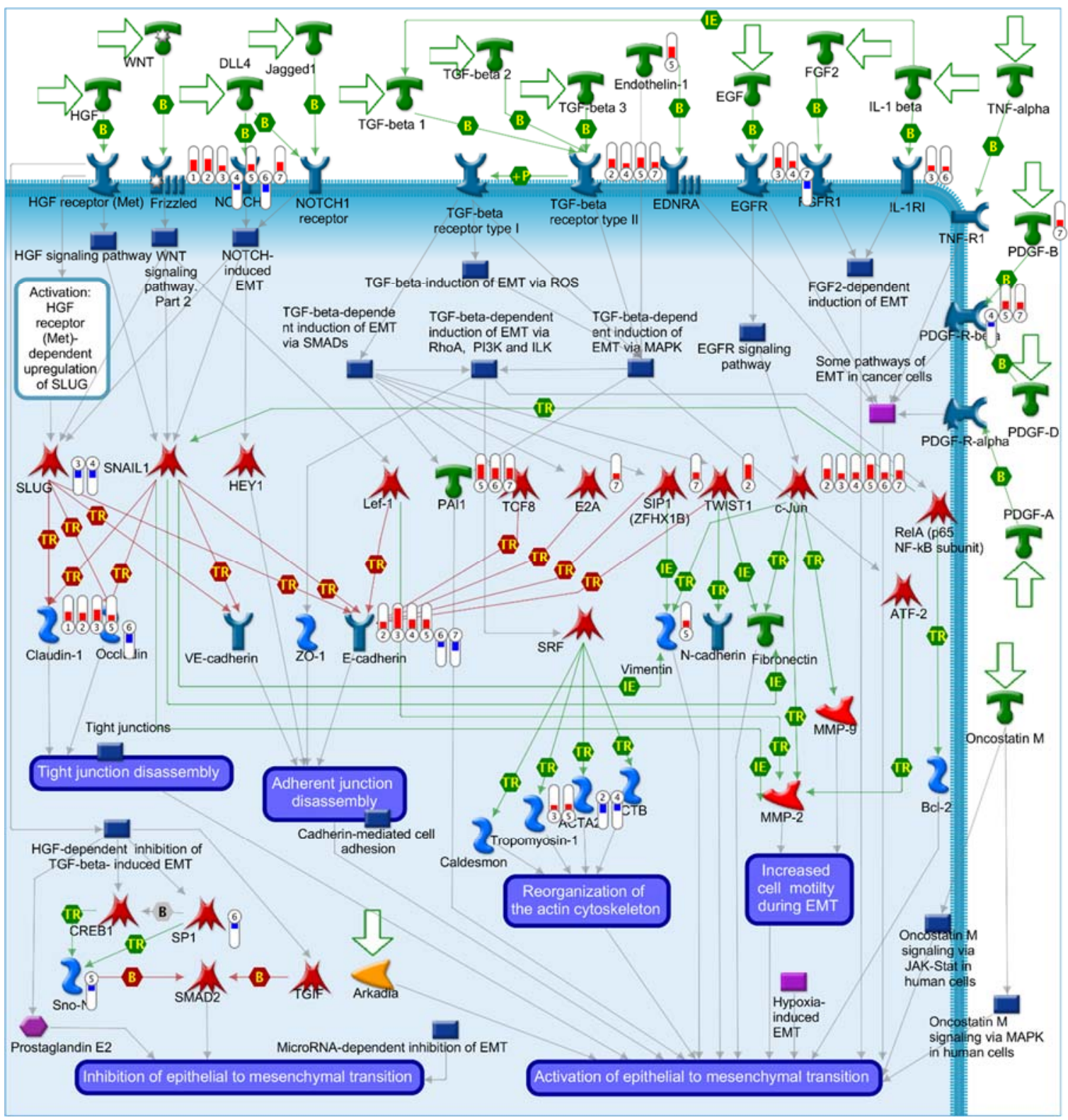

Figure 16. Claudin-1 and e-cadherin pathways related to EMT.

PCB exposure and PCB/HFD coexposures induced transcriptional changes in elements of signaling pathways related to epithelial to mesenchymal transition. The cell adhesion molecules claudin-1 and e-cadherin were differentially regulated in all or most conditions CVvsC20 (1), CVvsC2O0 (2), a HVvsH20 (3), HVvsH2O0 (4), C20vsH20 (5), C200vsH2O0 (6) and CVvsHV (7). Image generated using MetaCore ${ }^{\mathrm{TM}}$ from Thomson Reuters. 


\section{CHAPTER TWO: DISCUSSION}

PCB exposure induced transcriptional changes in mouse liver, and higher PCB exposure substantially increased the number of genes differentially transcribed compared to lower PCB exposure in the absence of dietary coexposure, the pathway characteristics of which are summarized in Figure 17. The addition of HFD as a coexposure greatly affected the number of differentially transcribed genes produced by PCB exposure: increasing the number and changing the distribution of engaged pathways. HFD itself produces many transcriptional changes in mouse liver, as have previously been described. PCB exposure modulates this effect, resulting in sets of differentially transcribed genes which overlap substantially. In the CVvsC20 comparison, nutritional and metabolic disease biomarkers were over-represented, and along with cardiovascular and vascular disease categories (also metabolic syndrome components), occupied five of the top ten most enriched categories in this group. It is interesting that these indicators were enriched in regular defined diet-fed animals exposed to PCBs, and that the

difference between vehicle and moderate PCB exposure was lost in HFD coexposure. This suggests that PCB and HFD exposure may converge on pathways regulating nutritional homeostasis, and that PCBs may contribute to metabolic dysregulation even in the absence of HFD.

With or without HFD coexposure, exposure to PCBs at $200 \mathrm{mg} / \mathrm{kg}$ increases enrichment in biomarkers of neoplastic disease. This is consistent with findings in previously reported rodent toxicity studies. Cancer outcomes have been a historic focus of PCB research, mainly due to the presence of dioxins and dioxin-like PCBs and dibenzofurans within the exposure mixture. Dioxins are potent carcinogens in mice, and have been classified as human carcinogens. Other than a recently reported increased prevalence of liver cancer among male Yusho (but not Yucheng) 


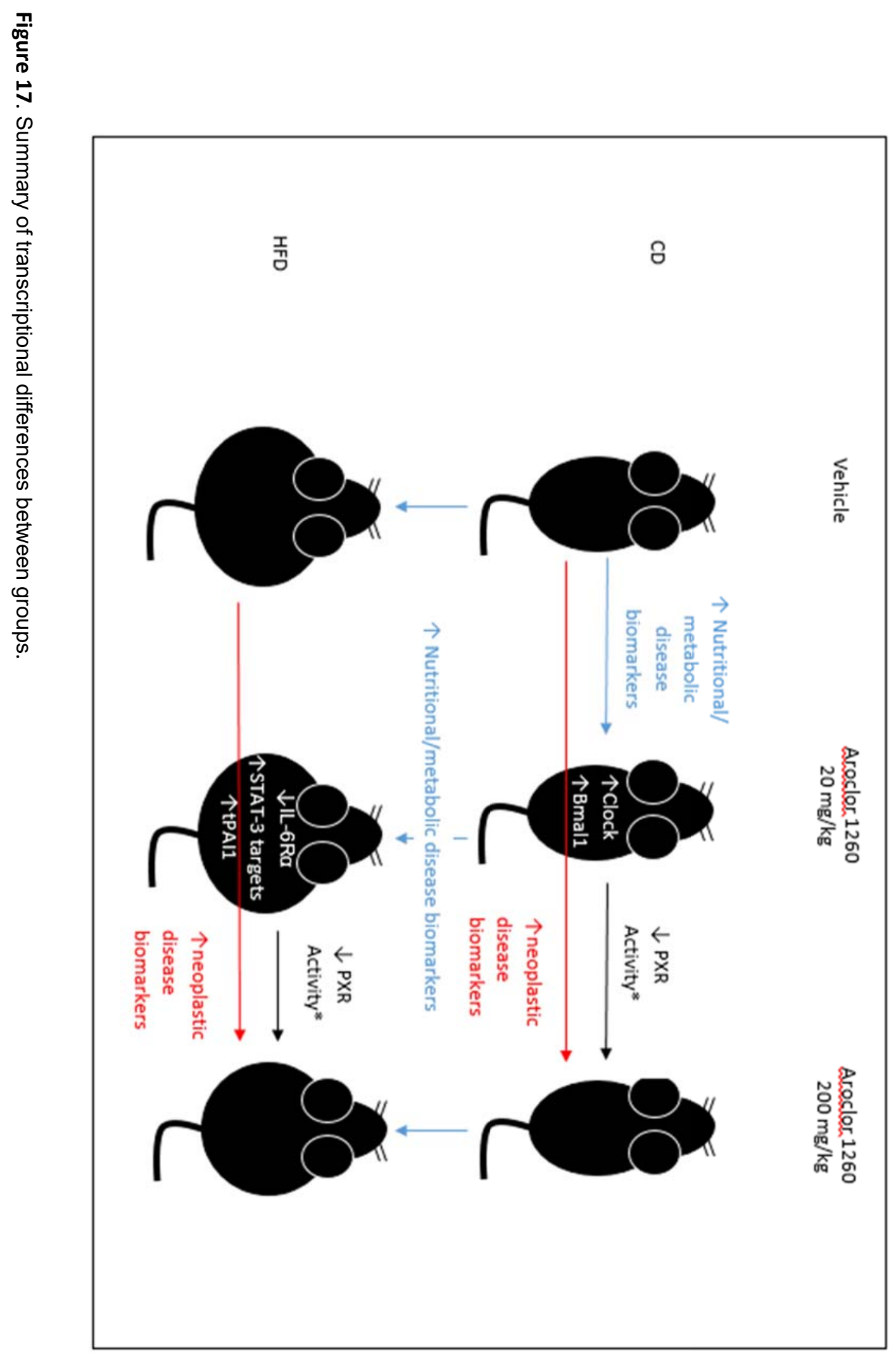


victims, however, the evidence linking PCB to cancer in humans has been underwhelming ${ }^{110}$. Interestingly, other than ovarian neoplasms in the HVvsH20 comparison, neoplastic disease categories were not in the top 10 disease enrichments by biomarker for either of the moderate exposure groups.

Our $20 \mathrm{mg} / \mathrm{kg}$ PCB exposure was based on the highest serum PCB level reported in the Anniston population ${ }^{23,79}$, which itself has a mean several times higher than that of the US average $^{148}$. The moderate PCB dose, therefore, is more representative of environmentallyrelevant human exposures, and we find that the distribution of diseases in these enrichments reflects this. Our analysis of the differential transcriptomes produced by liver tissue in these experiments in some ways recapitulated the proteomic and metabolic phenotype assessments. Within the HVvsH20 comparison, however, the most highly enriched disease category was IL-6 signaling (Table 45 and Figures 11-12). This is consistent with the findings of Wahlang et al., which indicated that the increased liver injury observed in the $\mathrm{HVvsH} 20$ model was not due to increased steatosis, but due to increased inflammation ${ }^{23}$. Increases in serum IL6 and tPAI1 protein were observed in the HFD/moderate exposure. In our transcriptomic analysis of liver tissue, pathways involving hepatocyte acute-phase response triggered by IL6 were observed in the moderate exposure (comparison $\mathrm{HVvsH} 20$ ).

We also saw enrichment in pathways and processes associated with control of circadian rhythm and rhythmic processes with moderate PCB exposure. Interestingly, in a 2015 report using metabolic cages to carry out metabolic phenotyping on PCB-exposed mice, our laboratory showed increased movement during the light (typical sleep) cycle in transgenic mice chronically exposed to $20 \mathrm{mg} / \mathrm{kg}$ Aroclor 1260 . These differences were observed only in mice with ablated constitutive androstane receptor (CAR) or pregnane and xenobiotic receptor (PXR), with a nonsignificant increase in wild-type mice. HFD feeding alone has been reported to alter the oscillatory patterns of circadian rhythm output genes in wild-type mice (Nampt, Acss2/ACSAdownreg in all CDvsHFD comparisons, Cyp2a5) ${ }^{136}$, but not the core clock genes, which are more resistant to reprogramming by nutritional challenge ${ }^{135,136}$. We also found differences in the 
transcription of clock output genes with HFD and HFD/PCB coexposure, but with moderate PCB exposure alone, we saw differences in transcription of the core clock genes as well, suggesting that PCB exposure and nutritional coexposures may impact these systems by different and potentially interacting mechanisms.

Although neoplastic disease biomarkers may not be captured by enrichment analysis in more realistic models of human environmental exposure, processes such as cell adhesion, cellcell communication, and pathways dictating cell identity changes through the processes of development and remodeling are over-represented in these models. The liver has extraordinary regenerative potential requiring plasticity in development and differentiation pathways even in adult hepatic tissue. Multiple pathways involved in development as well as repair were engaged in these models, including TGF-beta signaling, Wnt/ß-Catenin signaling, Hedgehog signaling, and Hippo signaling. Because these pathways are involved in development and regeneration, they also share common processes related to cell identity, orientation, and cell-cell recognition. Although pathway analysis shows engagement of these pathways in our animal model of environmental liver disease, it is unclear whether this engagement indicates regenerative response to ongoing PCB/HFD-dependent liver damage or modulation of basal or HFD-driven liver regeneration through xenobiotic receptor mediated PCB effect.

Responses to endogenous and exogenous organic compounds were over-represented among engaged processes both in the presence and absence of HFD coexposure. Responses to xenobiotics are thought to be mediated by xenobiotic receptors, of which AhR, PXR, and CAR are the prototypical example. In addition, xenobiotic receptors may also be activated by endogenous molecules, and crosstalk at multiple levels with receptors transducing hormone and metabolite sensing to control of intermediary metabolic processes. Among the direct transcriptional targets of these receptors with defined relationships curated by MetaCore ${ }^{\mathrm{TM}}$, few were differentially regulated, however, MetaCore ${ }^{\mathrm{TM}}$ software does not offer a comprehensive database of interactions for these receptors, and even some prototypical transcriptional effects of receptor-target interactions, such as Mus musculus PXR transcriptional activation of Cyp3a11, are not among the curated dataset. Canonical prototypical targets of PXR, including Cyp3a11, 
were up-regulated in the CVvsC20 condition. Alternative pathway analysis tools may offer more insight into the direct transcriptional effects of xenobiotic receptors in this model. It is interesting to note, however, that transcription factors which directly interact with and are inhibited by PXR showed a pattern consistent with increased engagement in the moderate exposure compared to the high exposures. Since these protein-protein interactions are ligand-dependent, it suggests that PXR (and potentially other xenobiotic receptors) may indeed be engaged, and that the mechanism of their effect in PCB-associated liver injury requires further study.

In summary, our RNAseq and subsequent pathway analysis of liver tissue from an animal model of PCB/HFD coexposure revealed several transcriptional changes which may be related to the observed phenotypic differences between groups. In particular, the enrichments in nutritional disease terms observed in the control diet animals exposed to PCBs may explain some of the differences in glucose handling which were previously reported for this experiment. ${ }^{23}$ Enrichments in IL-6 signaling with the moderate PCB/HFD coexposure may explain the increased histological evidence of liver injury observed only in this condition, while differential transcription affecting pathways involved in development and regeneration, as well as cell adhesion and cellcell recognition may provide some insight into differences in regenerative/fibrotic processes observed in the animal model. 
CHAPTER THREE

\section{PCB EXPOSURE INDUCES A DIFFERENTIAL TRANSCRIPTOME WHICH PARTIALLY OVERLAPS WITH THAT OF PROTOTYPICAL XENOBIOTIC RECEPTOR LIGANDS IN A MOUSE PRIMARY HEPATOCYTE MODEL}

Xenobiotic receptors are transcription factors which allow an organism to respond to chemical changes in the environment with appropriate metabolic adaptations. Although these changes are often circumscribed in the literature as encounters with environmental toxicants, it is important to recognize that xenobiotic receptors often respond to endobiotic signals as well, and are intimately involved in intermediary metabolism and inter-tissue communication along with the metabolism of xenobiotics. Even the arylhydrocarbon receptor (AhR), a classic focus of xenobiotic and toxicant research, affects ${ }^{149}$ and is affected by ${ }^{150}$, hormonal signaling pathways and the metabolic products of gut commensals. It is not surprising, therefore, that AhR and other xenobiotic receptors such as the pregnane and xenobiotic receptor (PXR) and the constitutive androstane receptor (CAR), have been implicated in metabolic derangements associated with toxicant exposures. Such derangements may manifest clinically and experimentally as components of metabolic syndrome, including steatohepatitis.

Nuclear receptors including PXR, CAR, farnesoid $X$ receptor $(F X R)$ and the liver $X$ receptor (LXR) are known to cross-talk at multiple levels, from common ligands ${ }^{151}$, binding partners, and cofactors ${ }^{152}$ to conserved response elements characterized by a DR $4^{153}$ or other shared motifs. This last intersection is most intriguing because while PXR and CAR are known to be sensors and effectors in xenobiotic metabolism, induction of the sterol-sensor LXR and the bile acid receptor FXR directly induce lipogenic gene targets, a phenomenon which is known to be modulated by CAR, PXR, and AhR. LXR induction, particularly as an off-target effect of 
pharmacotherapy, has been shown to lead to nonalcoholic fatty liver disease (NAFLD) ${ }^{154}$. Considering the interplay of these factors, it is not surprising that PXR, CAR, and LXR have been implicated along with the AhR in toxicant-associated steatohepatitis.

A further degree of complexity arises when we consider the effects of toxicant mixtures such as PCBs, which can be expected to affect several of these sensors simultaneously. PCBs are always encountered and bioaccumulated as mixtures of congeners, and persist at detectable levels in the serum of every adult in the United States ${ }^{37}$. The distribution of congeners found in the adipose tissue and serum of humans contains species which are known to modulate the activity of AhR, PXR, CAR ${ }^{19,34,35,155}$ and other receptors, and may, through crosstalking pathways, affect the transcriptional function of LXR. Because each of these receptors modulates gene targets related to steatohepatitis, an understanding of the contribution of each of these transcription factors to the overall PCB-modified transcriptome would assist with both risk assessment and the rational development of therapies.

Other groups have elucidated the subsets of the transcriptome modulated by AhR, PXR, and CAR individually in response to prototypical ligands. Findings have been intriguing for several reasons. Both in vivo and in vitro experiments suggest that for both $A h R$ and PXR, differential transcription of target genes appears to be ligand-specific ${ }^{156}$. Additionally, for primary hepatocytes, variations in cell isolation and culture techniques including culture time and plating substrate may drastically affect the expression levels of prototypical targets of AhR, CAR and PXR. In light of this, we sought to consolidate our assessment into a single primary murine hepatocyte model, examining the relationships between prototypical ligand responses and response to $\mathrm{PCBs}$ using RNAseq. We further sought to evaluate the PCB-dependent transcriptional output of this model for components of metabolic pathways related to steatosis and for gene ontology (GO) term enrichments within hepatic and metabolic disease categories. 


\section{CHAPTER THREE: MATERIALS AND METHODS}

Animals and primary cell isolation procedure

Male C57BL6 mice 8-10 weeks of age were purchased from Jackson Labs and allowed to acclimate for 1 week on a defined control diet (Harlan Teklad Cat TD.06416). Mice were deeply anesthetized with ketamine/xylazine, and the liver was flushed by retrograde perfusion first with a washing solution for 6 minutes, then with a buffered collagenase solution for 10 minutes. After perfusion, the liver was isolated and the gallbladder removed in a dish of ice-cold FBSsupplemented Waymouth's media. The liver was finely minced and the resulting suspension filtered through a $70 \mu \mathrm{m}$ cell strainer. Cells were immediately centrifuged, washed twice with ice cold HBSS, and selected by densitiy gradient centrifugation. The cell pellet was then resuspended in cold, supplemented Waymouth's media, and viability and cell count were determined. Cells were plated on collagen-coated 12-well plates and allowed to adhere. Adherent cells were exposed for 6 hours to $4 \mu \mathrm{l} /$ well vehicle (DMSO) or test substance solubilized in DMSO: Aroclor 1260 at $5 \mu \mathrm{g} / \mathrm{ml}$ or a prototypical ligand for $\mathrm{mPXR}$ - summarized in Table 58 (10 $\mu \mathrm{M}$ pregnane carboxynitrile, PCN), mCAR (10 $\mu \mathrm{M}$, TCPOBOP), LXR (1 $\mu \mathrm{M}$ GW3965 hydrochloride, GW) or AhR (50 $\mu \mathrm{M}$ benzathracene, BA). After exposure, media was removed and RNA was isolated by the RNAStat60 manufacturer's protocol.

RNAseq and gene transcription analysis

RNA samples were multiplexed using sequence barcoding, and sequenced single ended to 75 base pair reads using a NextSeq500 to an approximate read count of 40 million reads per 


\section{Table 58}

\section{$\underline{\text { Treatment summary }}$}

\begin{tabular}{|l|c|}
\hline Exposure & Anticipated Target \\
\hline DMSO & Control \\
\hline Benzanthracene (BA), $50 \mu \mathrm{M}$ & AhR \\
\hline Pregnane Carboxynitrile (PCN), $10 \mu \mathrm{M}$ & mPXR \\
\hline GW3965 $1 \mu \mathrm{M}$ & LXR \\
\hline TCPOBOP $\mu \mathrm{M}$ & mCAR \\
\hline Aroclor $1260(\mathrm{PCB}) 5 \mu \mathrm{g} / \mathrm{ml}$ & -- \\
\hline
\end{tabular}

Isolated primary mouse hepatocytes were exposed to Aroclor 1260 (a representative PCB mixture) or prototypical ligand for the above anticipated targets for $6 \mathrm{~h}$. 
sample. The bcl files produced were basecalled using Illumina's bcl2fastq software. All sequences produced were aligned to the mouse reference genome (GRCm38.83) using the alignment software TopHat, and transcript expression levels were calculated in FPKM units using Cufflinks $^{122}$. The quantification was guided by transcriptome annotation for the mouse downloaded from NCBI. Records corresponding to both mitochondrial and ribosomal RNA were removed (annotated as transcript_biotype rRNA or Mt_tRNA) to improve the accuracy of the transcriptome quantification. Differential analyses (FPKM units averaged from 4 replicates of each test vs. DMSO-treated controls) were performed using CuffDiff.

\section{Pathway and GO enrichment term analysis}

For each treatment, the fold changes and $p$-values for each differentially transcribed gene (indexed by Ensembl ID) were uploaded into MetaCore ${ }^{\mathrm{TM}}$ (Thompson Reuters) and recognized genes were associated with one or more MetaCore ${ }^{\mathrm{TM}}$-curated network objects. Experiments were analyzed using the MetaCore ${ }^{\mathrm{TM}}$ Enrichment Analysis Workflow tool, using a species $(M$. musculus) and tissue (liver) prefilter. With prefilters, the number of tags/genes was reduced to 87 targets (from 119).

QPCR validation of selected targets:

Targets for validation were selected based on RNAseq-indicated up-regulation and relevance to implicated transcription factors, pathways and disease processes. cDNA was generated using the QuantiTect Reverse Transcription kit (Qiagen \#205313) according to manufacturer recommendations. Multiplexed qPCR (target and GAPDH) was carried out on BioRad CFX384 system using the following TaqMan Gene Expression Array probes: Cyp1a2 (Applied Biosystems Mm00487224_m1/FAM), Cyp3a11 (Applied Biosystems Mm00731567_m1/FAM), Cyp2b10 (Applied Biosystems Mm01972453_s1/FAM) and GAPDH (Applied Biosystems, Mm99999915_g1/VIC). 


\section{CHAPTER THREE: RESULTS}

Aroclor1260 exposure at $5 \mathrm{ug} / \mathrm{mL}$ for $6 \mathrm{~h}$ induced differential transcription in primary mouse hepatocytes

Under these conditions, 123 targets were significantly differentially transcribed with Aroclor 1260 treatment vs. DMSO control (Figure 18 and Table 59). Among these, 68 were down-regulated $(F C>0.5)$, with 13 genes showing greater than 1 -fold reduction in transcription. Aroclor 1260 exposure resulted in up-regulation $(\mathrm{FC}<0.5)$ of 55 genes, with 3 genes upregulated more than 1 -fold.

In this model, the Aroclor 1260-dependent DTG sets partially overlapped with DTG sets produced by exposure to ligands for AhR, PXR, CAR, and $L X R$

Of the 123 targets within the PCB-DTG set, 41 (33\%) were not shared with the DTG sets produced by the prototypical ligands investigated, as shown in Figure 19. Most (67.5\%) of the genes within the PCB-DTG set overlapped with DTG sets produced by prototypical ligands for murine AhR, PXR, CAR, and/or LXR, as shown in Figure 20. The largest areas of overlap as a proportion of the total PCB-DTG set occurred with the PXR agonist PCN (65 targets in common with $\mathrm{PCB}$ exposure) and the AhR agonist BA (64 targets in common with PCB exposure). These two prototypical ligands produced by far the largest differential transcriptomes: 672 and 720 total DTGs, respectively, and the proportion of these transcriptomes which were also modulated by PCBs was relatively small $(9.7 \%$ and $8.9 \%$, respectively). The smallest area of overlap occurred between PCB and GW3960, a specific inducer of LXR, with only 14 targets differentially transcribed under both conditions. Although the direct murine CAR agonist TCPOBOP produced 


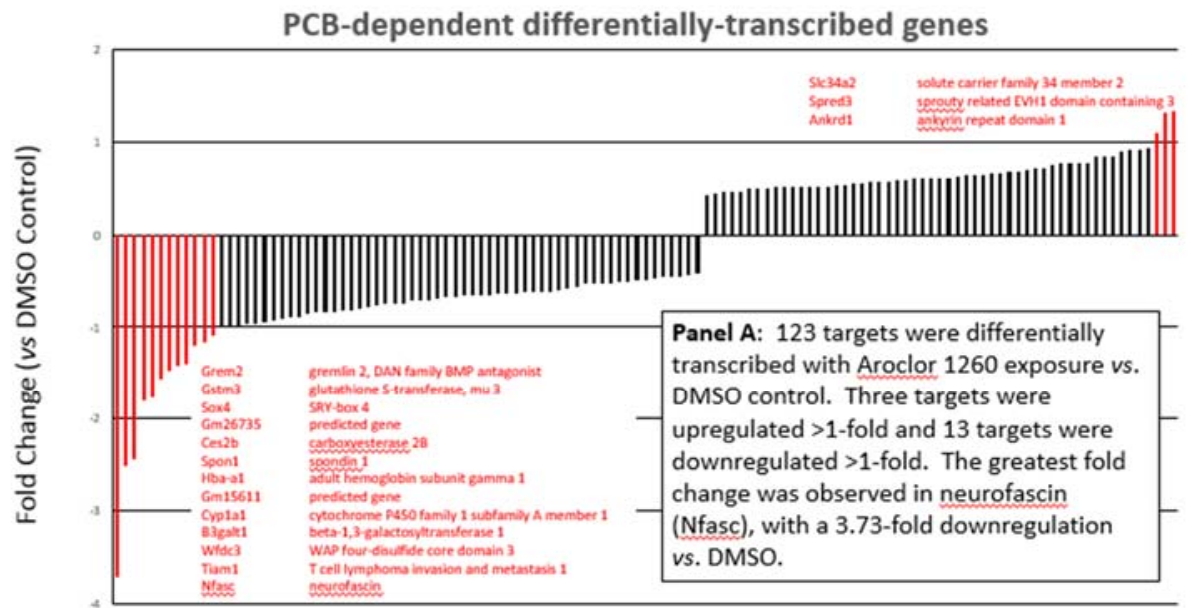

BA-dependent differentially-transcribed genes

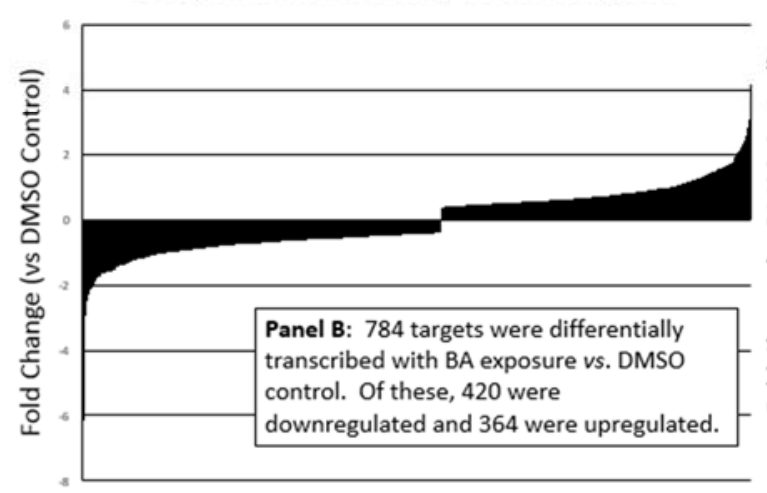

TCPOBOP-dependent Differentially Transcribed Genes

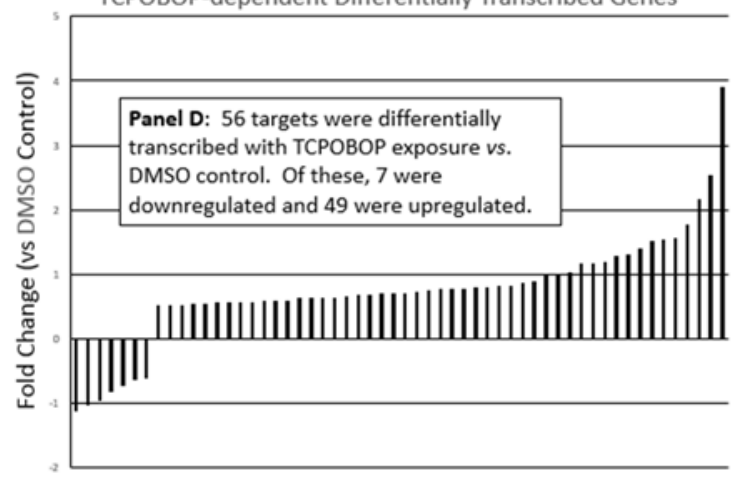

PCN-dependent differentially-transcribed genes

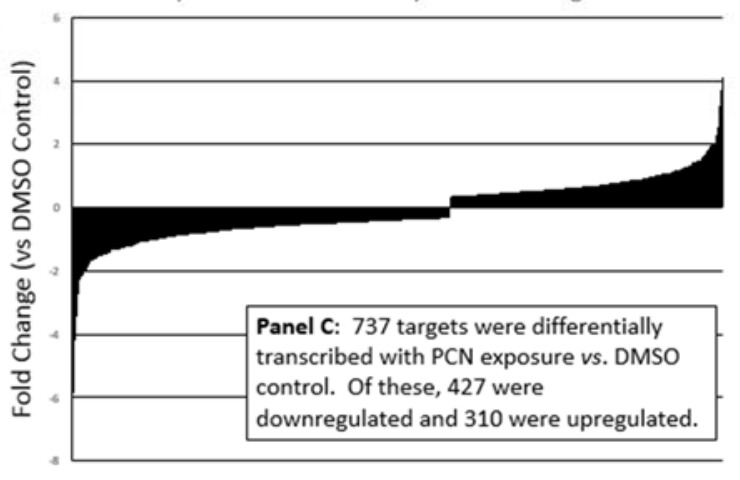

GW3965-dependent Differentially Transcribed Genes

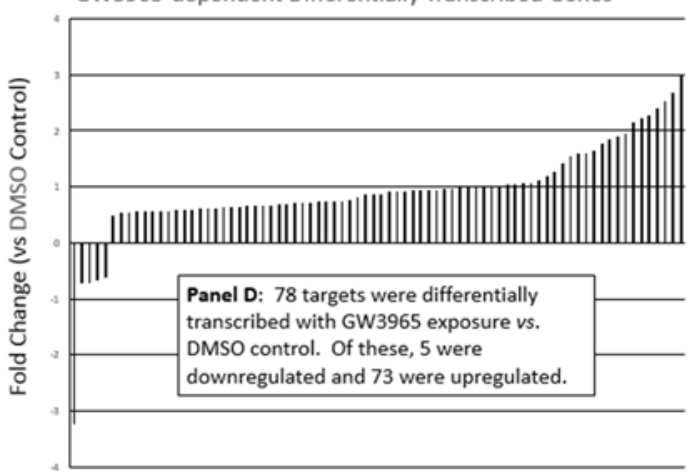

Figure 18. Histograms of differential transcriptomes produced by $\mathrm{PCB} /$ prototypical ligand exposure.

The differential transcriptome produced by Aroclor 1260 exposure in this model included both upregulated and downregulated targets, with fold changes ranging from -3.73 to +1.3 (Panel A).

Differential transcriptome FC ranges for prototypical ligands are shown in Panels B-E. 
Table 59

Targets differentially regulated with Aroclor 1260 Exposure

\begin{tabular}{|c|c|c|c|c|c|}
\hline Ensembl ID & Name & $\mathrm{FC}$ & Ensembl ID & Name & FC \\
\hline ENSMUSG00000026442 & Nfasc & -3.73 & ENSMUSG00000035171 & & -0.61 \\
\hline ENSMUSG00000002489 & Tiam1 & -2.51 & ENSMUSG00000011305 & Plin5 & -0.59 \\
\hline ENSMUSG00000076434 & Wfdc3 & -2.45 & ENSMUSG00000100798 & Gm19589 & -0.59 \\
\hline ENSMUSG00000034780 & B3galt1 & -1.81 & ENSMUSG00000036941 & Elac1 & -0.56 \\
\hline ENSMUSG00000032315 & Сур1a1 & -1.78 & ENSMUSG00000045502 & Hcar2 & -0.53 \\
\hline ENSMUSG00000084923 & Gm15611 & -1.57 & ENSMUSG00000028088 & Fmo5 & -0.53 \\
\hline ENSMUSG00000069919 & Hba-a1 & -1.47 & ENSMUSG00000035202 & Lars2 & -0.52 \\
\hline ENSMUSG00000038156 & Spon1 & -1.43 & ENSMUSG00000032310 & Cyp1a2 & -0.52 \\
\hline ENSMUSG00000050097 & Ces $2 b$ & -1.41 & ENSMUSG00000059325 & Hopx & -0.51 \\
\hline ENSMUSG00000097461 & Gm26735 & -1.20 & ENSMUSG00000030643 & Rab30 & -0.51 \\
\hline ENSMUSG00000076431 & Sox4 & -1.17 & UUSG00000019832 & Rab32 & -0.49 \\
\hline ENSMUSG00000004038 & Gstm3 & -1.10 & MUSG00000019726 & Lyst & -0.49 \\
\hline ENSMUSG00000050069 & Grem2 & -1.00 & MUSG00000039270 & Megf9 & -0.47 \\
\hline ENSMUSG00000041992 & Rapgef5 & -0.99 & MUSG00000049404 & Rarres1 & -0.46 \\
\hline ENSMUSG00000030909 & Anks4b & -0.97 & MUSG00000019866 & Aim1 & -0.46 \\
\hline ENSMUSG00000053168 & & -0.96 & ENSMUSG00000012428 & Steap4 & -0.45 \\
\hline ENSMUSG00000052305 & $\mathrm{Hbb}-\mathrm{bs}$ & -0.96 & MUSG00000023073 & Slc10a2 & -0.43 \\
\hline ENSMUSG00000101206 & Gm5266 & -0.95 & ENSMUSG00000018102 & Hist1h2bc & -0.42 \\
\hline ENSMUSG00000064128 & Cenpj & -0.92 & ENSMUSG00000038332 & Sesn1 & 0.42 \\
\hline ENSMUSG00000091572 & Vmn2r3 & -0.92 & MUSG00000021190 & Lgmn & 0.45 \\
\hline ENSMUSG00000052131 & Akr1b7 & -0.90 & MUSG00000056204 & Pgpep1 & 0.45 \\
\hline ENSMUSG00000031169 & Porcn & -0.89 & ENSMUSG00000000303 & Cdh1 & 0.46 \\
\hline 1USG00000057068 & Fam47e & -0.86 & VUSG00000041483 & Zfp281 & 0.46 \\
\hline ENSMUSG00000021260 & Hhipl1 & -0.84 & VUSG00000022512 & Cldn1 & 0.49 \\
\hline 1USG00000041132 & N4bp2I1 & -0.84 & VUSG00000037260 & Hgsnat & 0.50 \\
\hline USG00000055312 & Them7 & -0.84 & 0016382 & Pls3 & 0.50 \\
\hline USG00000038403 & $\mathrm{Hfe} 2$ & -0.83 & 0024773 & Atg2a & 0.51 \\
\hline USG00000078612 & & -0.81 & 21998 & Lcp1 & 0.51 \\
\hline 00020000 & Moxd1 & -0.80 & 35671 & Zswim4 & 0.51 \\
\hline 00030107 & Usp18 & -0.79 & 30790 & Adm & 0.51 \\
\hline 0061540 & Orm2 & -0.76 & 47777 & Phf13 & 0.52 \\
\hline 30089 & Slc41a3 & -0.75 & 4042 & Sik1 & 0.52 \\
\hline 57425 & Ugt2b37 & -0.75 & 3876 & Cgn & 0.52 \\
\hline 28359 & Orm3 & -0.74 & 014 & Pim1 & 0.53 \\
\hline 22032 & Scara5 & -0.72 & 350 & Gclc & 0.54 \\
\hline 63659 & Zbtb18 & -0.71 & 854 & Reps1 & 0.54 \\
\hline 46027 & Stard5 & -0.71 & 034 & Tcp11I2 & 0.55 \\
\hline 32715 & Trib3 & -0.69 & 351 & Cd63 & 0.56 \\
\hline 22244 & Amacr & -0.67 & 866 & Gm14420 & 0.56 \\
\hline 20553 & Pctp & -0.67 & 957 & Mmp14 & 0.57 \\
\hline 46794 & Ppp1r3b & -0.66 & 24486 & Hbegf & 0.58 \\
\hline 03762 & Adck4 & -0.65 & 19960 & Dusp6 & 0.59 \\
\hline 56148 & Rdh9 & -0.65 & 381 & Ugcg & 0.60 \\
\hline 73565 & Prr16 & -0.65 & 1USG0 & Ly96 & 0.61 \\
\hline ENSMUSG00000036181 & Hist1h1c & -0.64 & ENSMUSG00 & Plekhg3 & 0.61 \\
\hline ENSMUSG00000030827 & Fgf21 & -0.63 & ENSMUSG00 & Efna1 & 0.61 \\
\hline ENSMUSG00000031482 & Slc25a15 & -0.63 & 0021765 & Fst & 0.61 \\
\hline ENSMUSG00000107198 & Gm19619 & -0.63 & ENSMUSG00000024810 & II33 & 0.62 \\
\hline ENSMUSG00000022946 & Dopey2 & -0.62 & ENSMUSG00000040152 & Thbs1 & 0.64 \\
\hline ENSMUSG00000040562 & Gstm2 & -0.61 & ENSMUSG00000079427 & Mthfsl & 0.65 \\
\hline
\end{tabular}


Table 59 (Continued)

Targets differentially regulated with Aroclor 1260 Exposure

\begin{tabular}{|l|l|l|}
\hline Ensembl ID & Name & FC \\
\hline ENSMUSG00000078952 & Lincenc1 & 0.65 \\
\hline ENSMUSG00000044134 & Fam109a & 0.65 \\
\hline ENSMUSG00000024912 & Fosl1 & 0.66 \\
\hline ENSMUSG00000068299 & 1700019 G17Rik & 0.67 \\
\hline ENSMUSG00000078650 & G6pc & 0.68 \\
\hline ENSMUSG00000051439 & Cd14 & 0.70 \\
\hline ENSMUSG00000022218 & Tgm1 & 0.71 \\
\hline ENSMUSG00000017002 & Slpi & 0.71 \\
\hline ENSMUSG00000018774 & Cd68 & 0.75 \\
\hline ENSMUSG00000020607 & Fam84a & 0.76 \\
\hline ENSMUSG00000041836 & Ptpre & 0.77 \\
\hline ENSMUSG00000026728 & Vim & 0.77 \\
\hline ENSMUSG00000063531 & Sema3e & 0.77 \\
\hline ENSMUSG00000056515 & Rab31 & 0.84 \\
\hline ENSMUSG00000097073 & $9430037 G 07 R i k$ & 0.85 \\
\hline ENSMUSG00000097879 & Gm26869 & 0.85 \\
\hline ENSMUSG00000046733 & Gprc5a & 0.89 \\
\hline ENSMUSG00000032643 & Fhl3 & 0.91 \\
\hline ENSMUSG00000075707 & Dio3 & 0.92 \\
\hline ENSMUSG00000029304 & Spp1 & 0.93 \\
\hline ENSMUSG00000024803 & Ankrd1 & 1.09 \\
\hline ENSMUSG00000037239 & Spred3 & 1.31 \\
\hline ENSMUSG00000029188 & Slc34a2 & 1.34 \\
\hline
\end{tabular}

Gene targets differentially regulated with Aroclor 1260 exposure are shown. FC = fold change compared to DMSO control. 


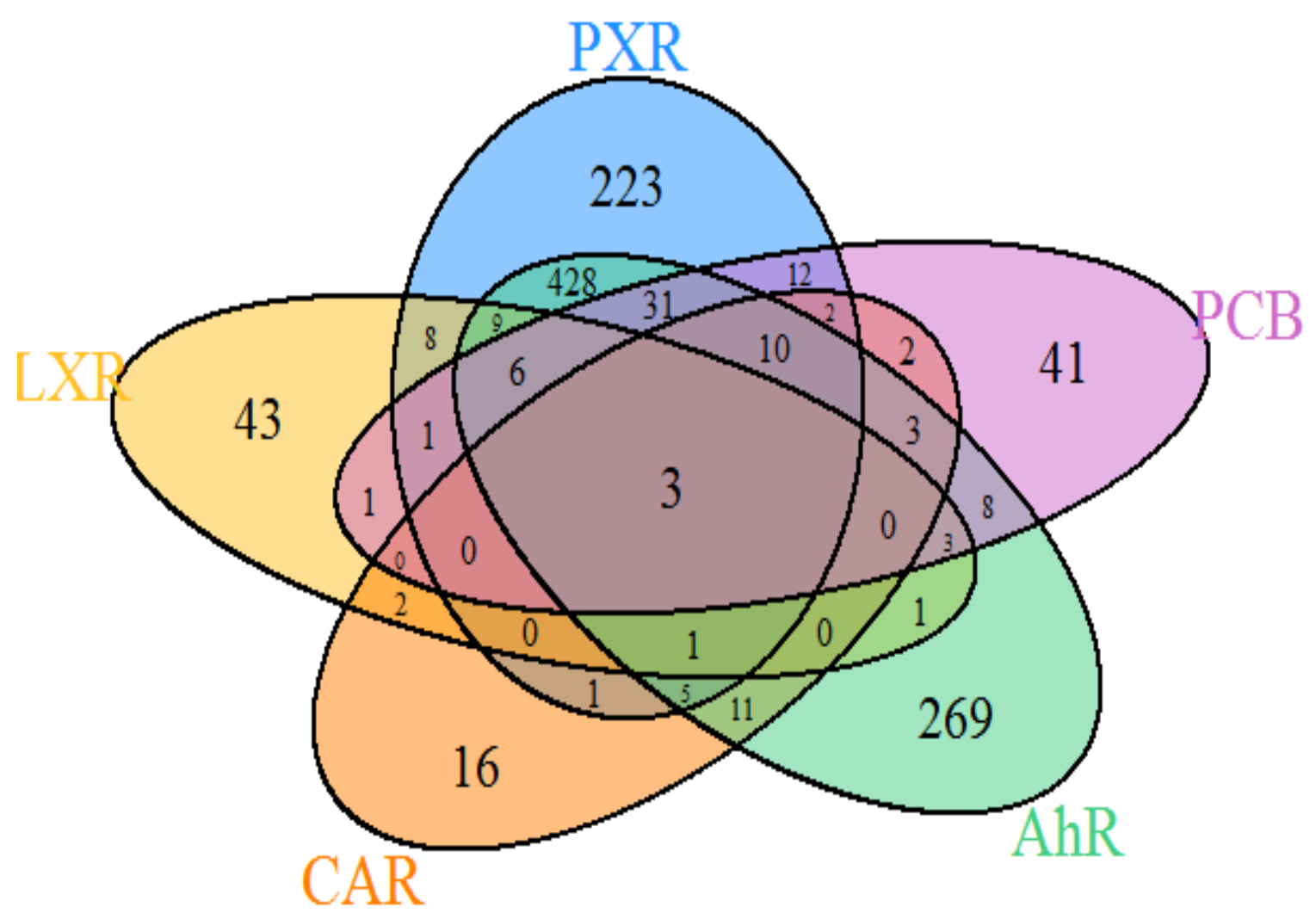

Figure 19. Venn diagram showing the overlapping differentially transcribed gene sets between PCB and prototypical ligand exposures.

Aroclor 1260 (PCB) exposure resulted in differential transcription of 41 (33.3\%) targets not attributable to ligand-activated transcription by any of the other four transcription factors investigated. This uniquely regulated section of the PCB-dependent transcriptome included unique of CREB1, SP1, and c-Myc. Several of the differentially transcribed targets, including SOX-4, Ptpre (Receptor-type Protein Tyrosine Phosphatase epsilon) and Sik-1 (salt-inducible kinase 1) are implicated in regulation of hepatic intermediary metabolism. 

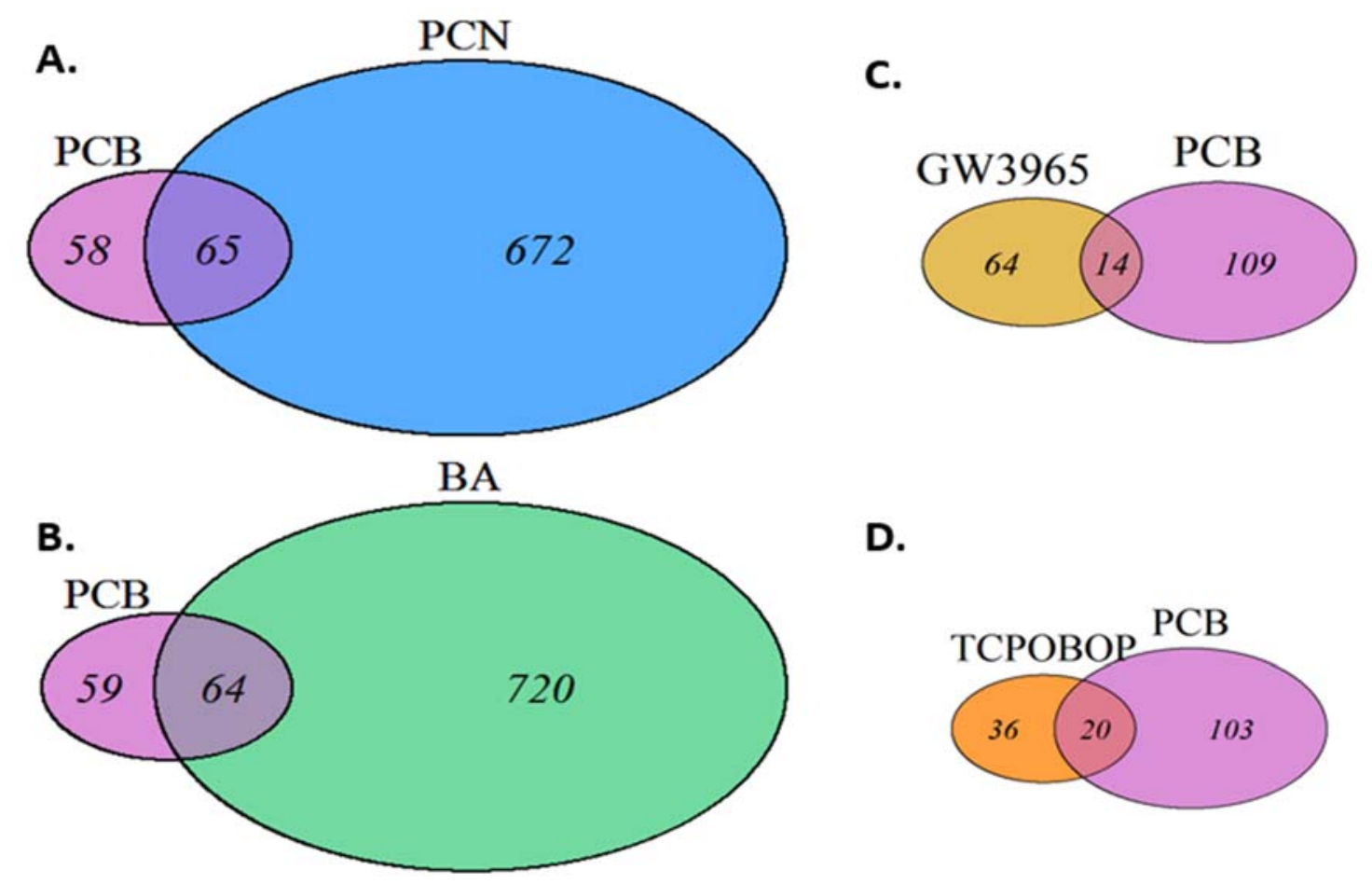

D.

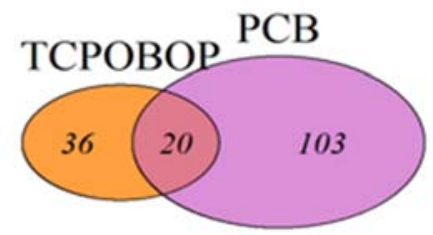

Figure 20. A portion of the PCB-DTG overlapped with DTGs for AhR, PXR, CAR, and LXR prototypical ligands. The differential transcriptome produced by Aroclor 1260 (PCB) exposure in this model overlapped with the differential transcriptome produced by each of the 4 prototypical ligands studied. The largest differentially-transcribed gene (DTG) sets were produced by A. pregnane carboxynitrile (PCN), a pregnane and xenobiotic receptor agonist and $\mathbf{B}$. Benzanthracene (BA), an arylhydrocarbon receptor agonist. These two prototypical ligands also produced the largest areas of overlap with PCB exposure (65 and 64 common DTGs, respectively). Two other prototypical ligands produced smaller total DTG sets. C. GW3965, a LXR agonist, produced a total differential transcriptome of 78 genes, 14 of which were also affected by PCB exposure. D. TCPOBOP, a direct constitutive androstane receptor (CAR) agonist, caused differential transcription of the smallest number of total genes (56), 20 of which were shared with PCB exposure. PCB exposure regulated a larger proportion of the total CAR differential transcriptome, however, than any other prototypical ligand studied $(36 \%$, vs $8 \%$ of BA-DTGs, $9 \%$ of PCN-DTGs, and $18 \%$ of LXR-DTGs). 
the smallest differential transcriptome (56 targets), 20 targets within this set were also targets of differential regulation by Aroclor 1260 exposure.

The direction of change in these common DTGs varied between treatment with Aroclor and prototypical ligands, but agreed with the direction of PXR and AhR differential transcription in most cases, as expected (Table 60), with more shared targets down-regulated than up-regulated. Of the 65 targets shared between PCB and PCN, 87.7\% agreed in direction. Exceptions included the gene target Usp18, which was moderately up-regulated in the PXR prototypical ligand condition and down-regulated with both Aroclor 1260 treatment and CAR prototypical ligand treatment. Of the 64 targets shared between $\mathrm{PCB}$ and $\mathrm{BA}, 85.9 \%$ agreed in direction. The six genes up-regulated with BA and down-regulated with Aroclor 1260 were all differentially regulated by at least one other transcription factor, and in fact, two of the three DTGs common to all 5 conditions were represented in this group (Cyp1a1 and Lars2). DTGs shared between GW3965/PCB and TCPOBOP/PCB included both up-regulated and down-regulated targets. In both cases, the up-regulated target sets were entirely concordant (6/6 for GW3965 and 10/10 for TCPOBOP, concurrently up-regulated/shared). $87.5 \%$ of genes were concordantly downregulated between GW3965 and PCB, while only half of the shared targets of TCPOBOP downregulated with PCB exposure agreed in direction. Although a tremendous amount of overlap existed between the conditions investigated, we were surprised to find that 41 DTGs $(33.3 \%)$ were unique to Aroclor exposure and are not shared with other prototypical ligand treatment (Figure 19 and Appendix Table 1), with 21 up-regulated and 20 down-regulated.

As expected, exposure to each of the prototypical ligands resulted in up-regulation of the corresponding prototypical target (Table 61). With Aroclor 1260 exposure, however, no significantly different transcription occurred (vs. DMSO control) in any prototypical target other than Cyp1a2, which was significantly down-regulated. This finding validates previous reports that while PCB exposure leads to induction of CAR, PXR, AhR and LXR prototypical targets in vivo ${ }^{23}$, these effects are not recapitulated in vitro. 


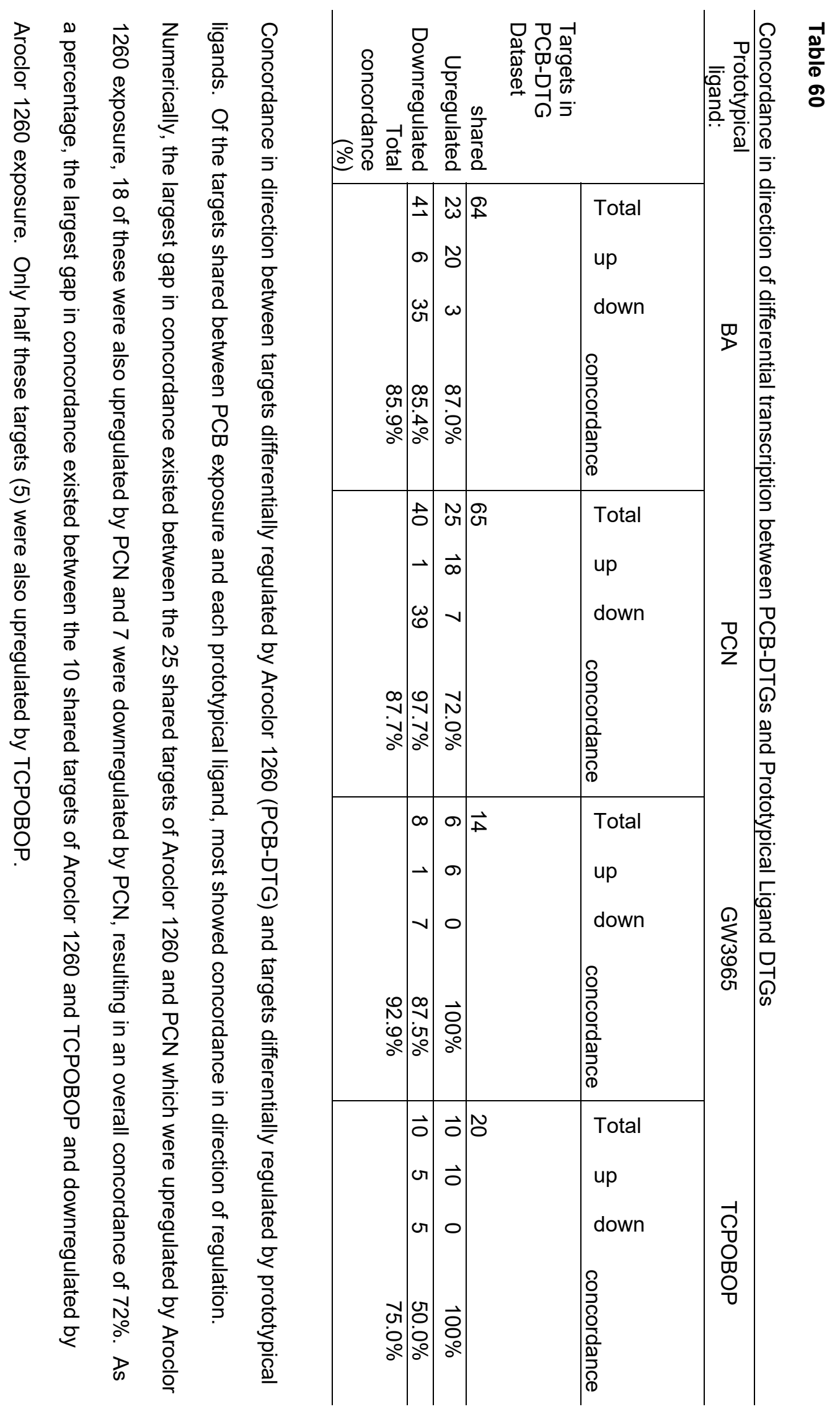




\section{Table 61}

Fold induction of prototypical targets for $A h R, P X R$, and CAR with prototypical ligand exposure

\begin{tabular}{lllll}
\hline & BA & PCN & TCPOBOP & Aroclor 1260 \\
\hline Cyp1a2 & $\mathbf{1 . 2 5}$ & -1.10 & 0.89 & -0.52 \\
Cyp3a11 & 0.41 & $\mathbf{0 . 9 7 0}$ & & \\
Cyp2b10 & & & $\mathbf{2 . 5 4}$ & \\
\hline
\end{tabular}

Fold change in each of the prototypical targets with exposure to prototypical ligands is shown. As expected, exposure to each prototypical ligand induced upregulation of the corresponding prototypical targets (shown in bold). Exposure to Aroclor 1260 did not result in significant change in any prototypical target except for Cyp1a2 (AhR target), which was downregulated. 
Aroclor1260 differentially transcribed genes are significantly enriched in pathway maps involved in cell identity and development, and in GO terms related to liver, endocrine, and metabolic disease

Initial output of in silico analysis using the MetaCore ${ }^{\mathrm{TM}}$ Enrichment analysis workflow identified 9 pathway maps significantly (FDR < .05) over-represented in the Aroclor1260 differentially transcribed gene set, shown in Table 62. Within these pathways, network objects related to differentiation or epithelial to mesenchymal transition (EMT) were enriched, particularly E-cadherin, vimentin, and claudin-1, which appeared separately or together in half of the top 10 enriched pathways. Pathways related to EMT were enriched for the PCB-DTG members. Figure 21 illustrates elements of this pathway.

Components of the PCB-DTG set were enriched in GO terms related to liver (31/4260 curated network objects, $p=6.0 \mathrm{E}-05, \mathrm{FDR}=4.7 \mathrm{E}-04)$, pancreatic $(27 / 3463$ curated network objects, $p=6.8 e-5$, FDR $=5.2$ e-4) and metabolic (29/3584 curated network objects, $p=1.6$ e-5, FDR $=2.0$ e-4) disease biomarkers. Within these sets, E-cadherin, vimentin and claudin-1 figured prominently, as did HB-EGF, AMACR, and osteopontin.

Aroclor1260 DTGs contain known or putative targets of transcription factors with known involvement in fatty liver disease and metabolic dysfunction. Transcription factor analysis of Aroclor 1260 indicated that large subsets of the PCB-DTGs set were predicted to be influenced by specific transcription factors including CREB-1, SP-1 and cMyc. Nuclear receptors such as HNF4- $\alpha$ and PPAR- $\gamma$, with known roles in steatosis and steatohepatitis, were also found to modulate the transcription of targets within this set. Significantly overconnected transcription factors are listed in Table 63. Notably, several of these transcription factors are direct or downstream targets of EGFR activation. 


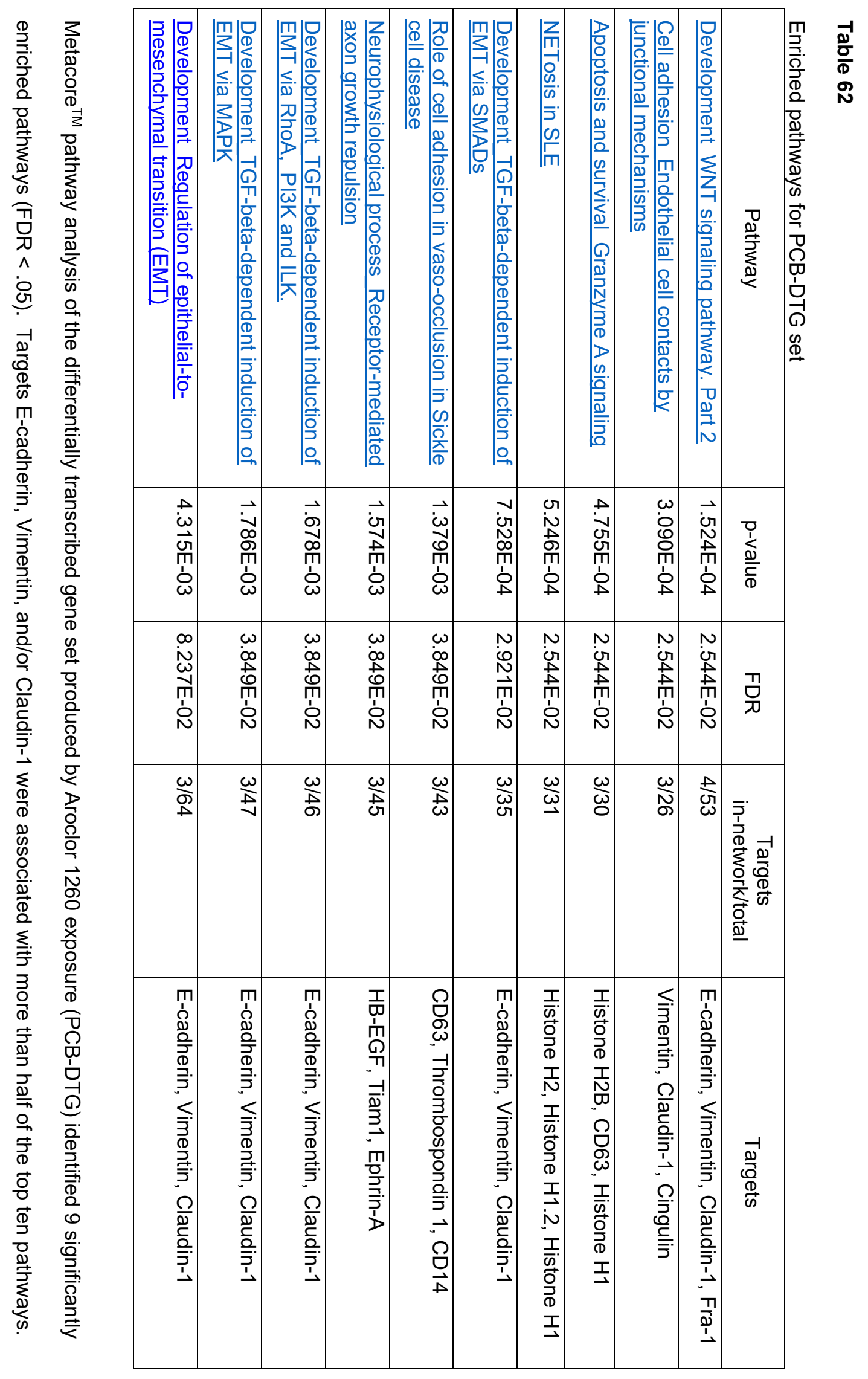




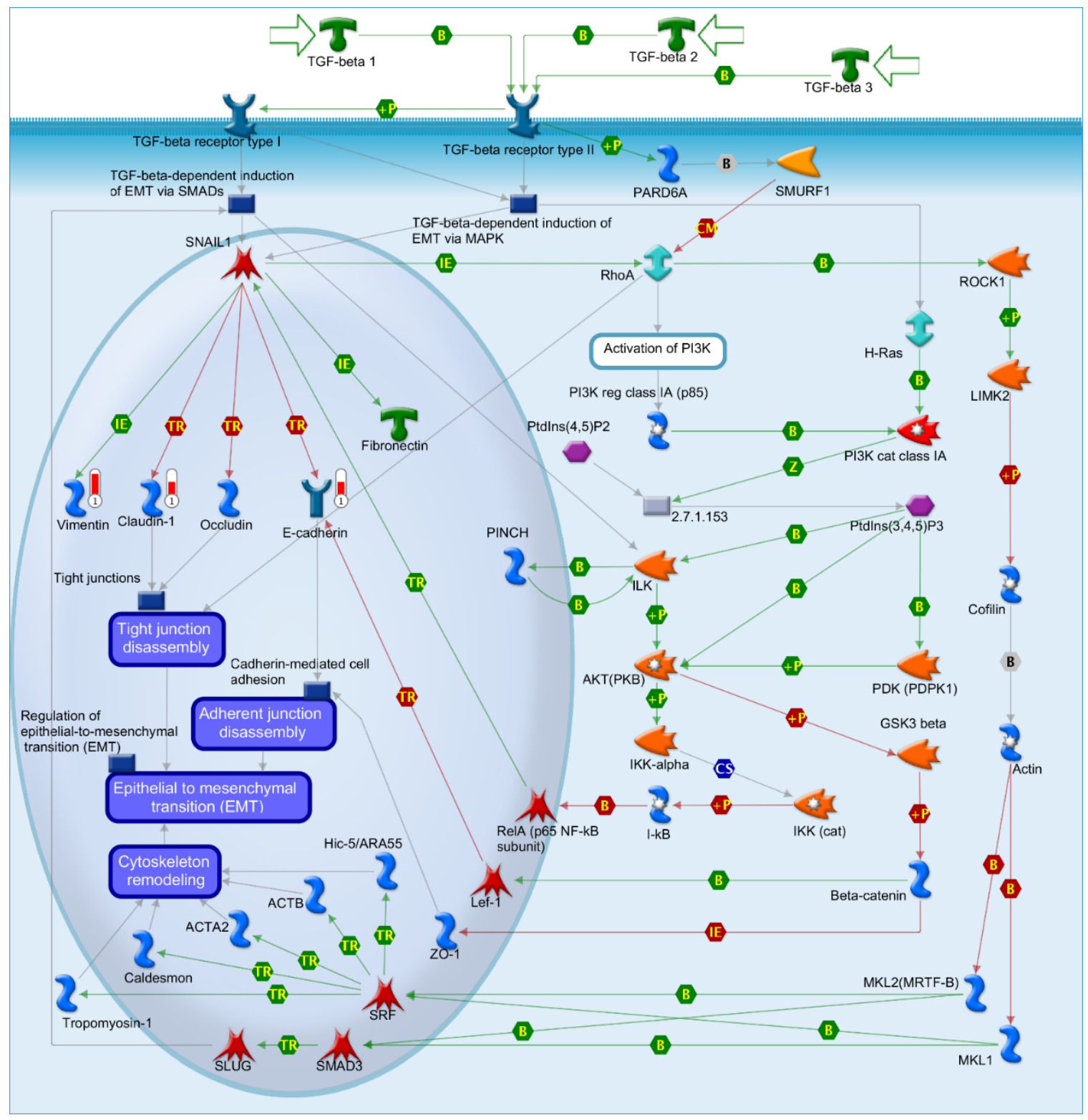

Figure 21. Pathways related to EMT were enriched in genes in the PCB-DTG set. The cell adhesion molecules claudin-1 and E-cadherin, as well as vimentin were important components of this pathway as well as others related to cell differentiation and tissue regeneration pathways. Image generated using MetaCore ${ }^{\mathrm{TM}}$ from Thomson Reuters. 


\section{Table 63}

Transcription factors with targets in the PCB-dependent differentially-transcribed gene set

\begin{tabular}{|l|l|l|l|}
\hline Network & $\begin{array}{l}\text { Seed } \\
\text { nodes }\end{array}$ & p-Value & zScore \\
\hline CREB1 & 33 & $3.050 \mathrm{E}-105$ & 212.83 \\
\hline SP1 & 23 & $8.940 \mathrm{E}-73$ & 176.55 \\
\hline c-Myc & 21 & $2.420 \mathrm{E}-66$ & 168.36 \\
\hline p53 & 20 & $3.910 \mathrm{E}-63$ & 164.12 \\
\hline C/EBPbeta & 19 & $6.230 \mathrm{E}-60$ & 159.76 \\
\hline Androgen receptor & 17 & $1.530 \mathrm{E}-53$ & 150.68 \\
\hline c-Jun & 15 & $3.570 \mathrm{E}-47$ & 141.01 \\
\hline ESR1 (nuclear) & 14 & $5.360 \mathrm{E}-44$ & 135.93 \\
\hline PPAR-gamma & 14 & $5.360 \mathrm{E}-44$ & 135.93 \\
\hline HNF4-alpha & 14 & $5.360 \mathrm{E}-44$ & 135.93 \\
\hline NANOG & 13 & $7.920 \mathrm{E}-41$ & 130.65 \\
\hline C/EBPalpha & 13 & $7.920 \mathrm{E}-41$ & 130.65 \\
\hline STAT3 & 12 & $1.150 \mathrm{E}-37$ & 125.15 \\
\hline HIF1A & 12 & $1.150 \mathrm{E}-37$ & 125.15 \\
\hline GATA-3 & 12 & $1.150 \mathrm{E}-37$ & 125.15 \\
\hline ATF-4 & 12 & $1.150 \mathrm{E}-37$ & 125.15 \\
\hline c-Fos & 11 & $1.660 \mathrm{E}-34$ & 119.4 \\
\hline ReIA (p65 NF-kB subunit) & 11 & $1.660 \mathrm{E}-34$ & 119.4 \\
\hline Bcl-6 & 10 & $2.340 \mathrm{E}-31$ & 113.37 \\
\hline SMAD3 & 10 & $2.340 \mathrm{E}-31$ & 113.37 \\
\hline VDR & 10 & $2.340 \mathrm{E}-31$ & 113.37 \\
\hline IRF1 & 10 & $2.340 \mathrm{E}-31$ & 113.37 \\
\hline SMAD2 & 10 & $2.340 \mathrm{E}-31$ & 113.37 \\
\hline Oct-3/4 & 10 & $2.340 \mathrm{E}-31$ & 113.37 \\
\hline JunD & 9 & $3.250 \mathrm{E}-28$ & 107.02 \\
\hline GCR & 9 & $3.250 \mathrm{E}-28$ & 107.02 \\
\hline FKHR & 9 & $3.250 \mathrm{E}-28$ & 107.02 \\
\hline PPAR-alpha & 8 & $4.430 \mathrm{E}-25$ & 100.27 \\
\hline TCF7L2 (TCF4) & 8 & $4.430 \mathrm{E}-25$ & 100.27 \\
\hline p63 & $4.430 \mathrm{E}-25$ & 100.27 \\
\hline
\end{tabular}

Transcription regulation pathway analysis was performed on the PCB-DTG dataset without prefilters. 


\section{CHAPTER THREE: DISCUSSION}

Classically, the transcriptional effects of PCB exposure have been attributed entirely to ligand-dependent activation of AhR, PXR and CAR. We expected that the subset of genes differentially transcribed with Aroclor 1260 exposure in this acute primary hepatocyte model would overlap almost entirely with the transcriptional output of prototypical ligands for these three receptors. We were therefore surprised to discover that fully $1 / 3$ of DTGs were unique to Aroclor1260 in this system, possibly due to the transcriptional effects of another xenobiotic receptor or by an indirect mechanism. Our laboratory has recently reported that EGFR is also a target of inhibition by both Aroclor1260 and individual PCB congeners. PCBs therefore would be expected to function as an indirect CAR activator in both mice and humans by the same mechanism as phenobarbital ${ }^{21}$.

The canonical cytochrome P450 targets of CAR and PXR activation cyp2b10 and cyp3a11 did not appear among the RNA-seq derived PCB-DTG gene set, while cyp1a1 and cyp1a2 (prototypical targets of AhR induction) were both down-regulated in the PCB-DTG set. Interestingly, cyp1a1 and cyp1a2 were also down-regulated by PCN treatment in this model. Regulation of cyp1a1 was particularly complex: up-regulated by inducers of CAR and LXR as well as AhR and down-regulated by PXR induction. We conclude that differential regulation of the proportion of PCB-DTGs overlapping with targets of the evaluated receptors is complex and may be influenced by the combined effects of these and other xenobiotic receptors.

The targets most strongly up-regulated with Aroclor 1260 exposure, slc34a2 (+1.34-fold vs. control) and spred3 (+1.31-fold vs. control) were uniquely up-regulated by PCBs in this model. Although slc34a2 was also a target differentially regulated by PCN exposure, it was downregulated in that condition and unchanged with exposure to other prototypical ligands. The targets 
most strongly down-regulated with Aroclor 1260 exposure, nfasc (-3.73-fold vs. control) Tiam1(0.51 -fold vs. control) and wfdc3 (-2.45-fold vs. control) were also strongly down-regulated by PCN treatment (-4.20, -2.73 , and -2.13 -fold vs. control, respectively). PXR is involved in differential regulation of transcription related to PCB exposure, however, it seems more involved in inhibition of these targets than induction.

Several of the genes differentially transcribed with PCB exposure in this model are differentially transcribed in the incidence or progression of liver disease or liver disease biomarkers. Osteopontin (Spp), which was up-regulated with PCB (+0.93-fold vs. control) is upregulated in NASH fibrosis has been shown to oppose EMT in liver progenitor cells ${ }^{157}$. Four And A Half LIM Domains 3 (FHL3), which was up-regulated with PCB (+0.91-fold vs. control) affects transcription of tPAl1, a fibrosis biomarker up-regulated in PCB-exposed humans. RAB31, a member RAS Oncogene Family, which was up-regulated with PCB (+0.84-fold vs. control), is associated with invasive/metastatic characteristics and worse prognosis in $\mathrm{HCC} .{ }^{158}$. Semaphorin 3E (Sema3e), which was up-regulated in PCB exposure (+0.77 vs. control) is expressed by damaged hepatocytes and secreted, inducing contraction of sinusoidal epithelial cells and activation of stellate cells. Chronic exposure to Semaphorin $3 \mathrm{E}$ in a mouse model of $\mathrm{CCl}_{4}$-induced chronic heptatitis lead to disorganized sinusoidal regeneration and exacerbated fibrosis. ${ }^{159}$ Cytochrome P450 1a1 (Cyp1a1), which was down-regulated (-1.78-fold vs. control) is a prototypical target of the arylhydrocarbon receptor and is up-regulated in animal models of TCDD-induced nonalcoholic steatohepatitis. ${ }^{160}$ Interestingly, in this model, although it was upregulated by prototypical ligands of LXR and CAR in addition to AhR, it was strongly downregulated by PXR ligand exposure. Carboxyesterase 2 (Ces2b), which was down-regulated in our model (-1.41-fold vs. control), and in the livers of NASH patients. Experimental ablation of Ces2b in a mouse model causes ER stress in hepatocytes and stimulates lipogenesis in an SREBP-1dependent manner. ${ }^{161}$ Aldo-keto reductase family 1, member B7 (Akr1b7), which was downregulated in our model (-0.90-fold vs. control) is enriched in adipose stromal vascular fraction, but not in mature adipocytes. In a mouse model, Akr1b7 KO increased basal adiposity (adipocyte 
hyperplasia and hypertrophy) with diet-independent development of liver steatosis and insulin resistance. ${ }^{162}$ Hemochromatosis, type 2 (Hfe2), which was down-regulated in our model (-0.83 vs. control), is expressed at significantly lower levels in NAFLD patients vs. those with no disease. Because $\mathrm{Hfe} 2$ is involved in iron-sensing pathways, it is hypothesized that lower levels of $\mathrm{Hfe} 2$ are related to the dysregulated iron-sensing pathways leading to iron overload in NAFLD. ${ }^{163}$ Thrombospondin-1 (Thbs 1$)$, was up-regulated in our model (+0.64 vs. control). Transcription of Thrombospondin-1 is increased in individuals with chronic liver disease and in mouse models of liver fibrosis. ${ }^{164}$ Interleukin 33 (II33) was up-regulated in our model (+0.62 vs. control), and is elevated in the serum of NASH patients, increasing with increasing histologic severity. ${ }^{165}$ Several of the genes differentially transcribed with PCB exposure in this model affect pathways of interest. Sprouty-related protein with EVH-domain 3 (Spred-3), which was upregulated with $\mathrm{PCB}\left(+1.31\right.$-fold vs. control) potently inhibits ERK1/2 signaling ${ }^{166,167}$. Four And A Half LIM Domains 3 (FHL3) physically interacts with SMAD proteins (TGF $\beta$ ) pathway. ${ }^{168}$ RAB31 is associated with modulation of EGFR trafficking. It physically interacts with EGFR and its overexpression increases early-late endosome trafficking and degradation of EGFR. ${ }^{169,}{ }^{170} \mathrm{~T}$ lymphoma invasion and metastasis 1 (Tiam1), which is strongly down-regulated with PCB (-2.51fold vs. control) is differentially regulated in hepatocellular carcinoma and pancreatic cancer tissues. ${ }^{171,172}$ In pancreatic cancers, the Par3 interacts with Tiam1 to affect tight junctions by downregulating Claudin-1 and Occludin. ${ }^{172}$ Tiam1 is also a target suppressed by several miRNAs. ${ }^{173,174}$ Sustained ectopic exposure to Sema3E (up-regulated by PCB exposure in this model) has been found to result in disorganized regeneration of liver sinusoids, contributing to fibrosis, in a mouse model of liver regeneration. ${ }^{159}$ SRY (sex determining region Y)-box 4 (Sox-4), was down-regulated with PCB exposure in our model (-1.17-fold vs. control). In HepG2 cells, inhibition of Sox-4 blocks caspase-1-dependent apoptosis. ${ }^{175}$ Glutathione S-transferase, mu 3 (Gstm3), which was down-regulated with PCB exposure in our model (-1.10-fold vs. control) is involved in glutathione-dependent clearance of xenobiotics (Phase II metabolism). ${ }^{176}$ Protein Tyrosine Phosphatase, Receptor Type E (Ptpre) was up-regulated with PCB exposure, and functions to modulate insulin signaling in hepatocytes and skeletal muscle by suppressing 
phosphorylation of targets down-stream of insulin signaling such as Akt, ERK and GSK3. ${ }^{177}$ Porcupine homologue (Porcn), which was down-regulated (-0.89-fold vs. control) is an ER protein essential for the processing of Wnt proteins, and its inhibition inhibits Wnt signaling. ${ }^{178}$

Lastly, several of the genes differentially transcribed with PCB exposure in this model are affected by pathways of interest. $\mathrm{Na}^{+}-\mathrm{P}_{\mathrm{i}}$ Cotransporter $\mathrm{NaPi}-\mathrm{Ilb}$ is regulated by glucocorticoid, estrogen, EGF, aging, ${ }^{179}$ dietary phosphate status, hormones like parathyroid hormone, 1,25 $\mathrm{OH} 2$ vitamin D3 or FGF23 ${ }^{180}$. Expression of Gstm is induced by wnt/ $\beta$-catenin activation and inhibited with Ras activation. ${ }^{181}$ Aldo-keto reductase family 1, member B7 (Akr1b7) is induced in the liver by activation of FXR ${ }^{182} C A R, P X R$, and $L X R$ via direct interaction with DR4 elements in the promoter, and that the influence of PXR/LXR is additive. ${ }^{183}$

The MetaCore ${ }^{\mathrm{TM}}$ curated GO processes most significantly enriched in genes affected by Aroclor 1260 exposure included several recurring objects with roles in Wnt signaling, cell adhesion and/or EMT: pathways which are altered in the progression of fatty liver disease ${ }^{184}$. Of these, up-regulation of Claudin-1 was unique to Aroclor 1260 exposure and although more than one prototypical ligand induced differential expression of E-cadherin (PCN and BA), it was upregulated only with PCB exposure. Vimentin, in contrast, was up-regulated with PCN and TCPOBOP as well as PCB exposure. Importantly, EMT in hepatocytes is characterized by a decrease in E-cadherin and claudin-1, corresponding to increased inhibition by SNAIL/SLUG signaling down-stream of TGF $\beta$. EMT in hepatocytes is characterized by decreases in Ecadherin and claudin-1, and increased Vimentin, however, with PCB exposure, we saw increases in expression of both epithelial (E-cadherin and Claudin-1) biomarkers, along with increased Vimentin (a mesenchymal biomarker). Although decreases in expression are associated with mesenchymal change, exogenously expressed Claudin-1 promotes the acquisition of mesenchymal characteristics in hepatocellular carcinoma ${ }^{185}$. Decreased Claudin-1 and Ecadherin in Increases in Claudin-1 expression characterize HUVEC cells undergoing differentiation to endodermal and hepatic stages. ${ }^{186}$

In the context of liver damage and regeneration, the designation of "epithelial" or "mesenchymal" refers not to the inflexible characteristic of lineage (origin) but rather, the plastic 
cellular organization and metabolic commitments underlying tissue-level function ${ }^{187}$. Epithelial cells are characterized by tight cell-cell contacts and apico-basal polarity. In epithelioid adult liver parenchymal cells, (hepatocytes and cholangiocytes), these characteristics are critical to their function as a selectively permeable, transformative metabolic layer receiving input from enterohepatic circulation (sinusoidal/basolateral) and contributing modified output to systemic circulation and the biliary system (cannalicular/apical). The levels, ratio, and distribution of claudins, members of the main transmembrane protein family forming hepatocyte intercellular tight junctions form the structural underpinning of this polarity/permeability, and so define specific identity and function of the cell.

In contrast, traits associated with mesenchymal cells include a lack or loss of polarity and loss or differential organization of the protein architecture required to maintain cell-cell and cellECM contacts. A transition from epithelial to mesenchymal or mesenchymal to epithelial qualities involves the gradual loss of characteristic markers of one state and gain of markers characteristic of the other, with transitioning cells often expressing some of each. In this case, high E-cadherin and claudin would be indicative of epithelioid character, while Vimentin is typically a mesenchymal marker ${ }^{140}$.

In summary, in primary hepatocytes, differential transcription induced by PCB exposure cannot be completely explained by direct ligand-induced transcriptional activity of the AhR, PXR, or CAR by congeners within the Aroclor 1260 mixture. This is surprising, considering the vast amount of research that has focused on these three receptors as mediators of PCB effect. AhR and PXR had the largest areas of overlap, however, the direction of differential transcription was often opposite between Aroclor 1260 DTGs and the DTGs produced by treatment with these prototypical ligands, particularly PXR. LXR, which is unlikely to be bound by congeners within the mixture, is likely to crosstalk with PXR and CAR at the level of chromatin binding, as it recognizes the same DR-4 motif response element. LXR is often implicated as a key transcription factor involved in the development of steatosis leading to steatohepatitis, however, transcriptional changes due to direct ligand activation of LXR did not have a large overlap with the Aroclor 1260dependent differential transcriptome either. We conclude from the information in this model that 
other transcription factors are likely responsible for a large portion of the transcriptional changes observed with PCB exposure.

Transcription factors that appear to be involved in PCB-dependent transcription are often directly or indirectly influenced by EGFR-mediated cell signaling via phosphorylation, consistent with our recent report that PCBs inhibit EGFR signaling ${ }^{20}$. MetaCore ${ }^{\mathrm{TM}}$ pathway analysis of the PCB-DTG set indicated enrichment in biomarkers associated with liver disease, and components pathways known to be involved in the progression of fatty liver disease. Targets uniquely modified by PCB exposure in this model include markers of epithelioid character and hepatocyte polarity, as well as ECM modifiers. Together, these suggest that at least some of the diseases associated with PCB exposure are due to differential transcription mediated by one or more receptors. More work is needed to determine the relevance of engaged pathways suggested by this preliminary investigation. 


\section{CONCLUSIONS}

The study of human disease related to environmental exposures requires a multidisciplinary approach unique in biomedical research. It requires a balance between overly reductionist approaches that ignore poorly understood pathways and massive, non-mechanistic association studies which muddy the waters and yield unapproachable data. The experimental approaches to PCB research suffer from both problems, resulting in a body of literature that is measured "by the pound, not by the page" 39 : a massive body of exposure data with mechanistic assumptions based on changes to one or two transcriptional targets of one or two xenobiotic receptors. The overall result of this status is that even 80 years after publication of the first paper linking PCB exposure to human disease, the mechanisms of PCB-related diseases are poorly understood.

The Cave laboratory began with a study of the people exposed to PCBs - every adult in the US population, represented by the massive, cross-sectional National Health and Nutrition Evaluation Survey, or NHANES. That study demonstrated that PCBs are ubiquitous and associated with liver injury in the general population ${ }^{37}$. A logical next step was then an epidemiological evaluation of liver disease in a population with a relatively high exposure to environmental PCBs - that of Anniston, Alabama, already under investigation to determine whether PCB exposure was associated with multiple metabolic diseases.

Both the positive and negative findings of our study were striking and novel - although nonalcoholic fatty liver disease in Anniston was clearly elevated compared to expected prevalence in the general US and worldwide population, the mechanistic biomarkers employed showed that the overall level of the PCB exposure represented by the sum of all congeners was 
not correlated with the severity of liver injury or inclusion in a liver disease category in the overall population.

We considered two potential reasons for this observation which were not mutually exclusive. First, the Anniston population represents a complex, but not atypical, exposure situation, which was one of the acknowledged limitations of the study. Chemical exposures alone included not only PCBs, but also heavy metals and pesticides, many of which are associated with liver or metabolic disease ${ }^{37,121,188}$, while nutritional coexposures inferred from the high prevalence of obesity/overweight in this population could contribute independently to fatty liver disease. A second possibility is that, like other endocrine/metabolism-disrupting chemical exposures ${ }^{189}$, increasing PCB dose could result in a nonmonotonic dose-response (NMDR) with respect to damage biomarkers - not steadily increasing or decreasing with increasing exposure concentration. At the cellular level, differential engagement of crosstalking mechanisms may result in different output at different exposures. At the organismal level, PCB effects on multiple systems must be considered: suppressive effects on inflammatory pathways could lead to higher overall liver damage indicators at a lower exposure level, interference with circadian control could contribute to hyperphagy and decreased energy expenditure, while effects on hormonal signaling could communication between concerted systems. For this last consideration, nutritional coexposures play a role as well: direct effects of PCBs to reduce insulin output could limit the compensatory role of the pancreas in glucose allostasis, putting more stress on connected systems including the liver.

The animal study previously reported by Wahlang et al..$^{23}$ was an attempt to disambiguate the effects of PCB exposure level and nutritional coexposure in an animal model of chronic PCB/HFD coexposure. We integrated our epidemiological study with our animal model by comparing serology and phenotype in both studies. The condition represented by animals coexposed to HFD and moderate PCBs (20 mg/kg) was designed to most closely model the Anniston exposure, and, indeed, the phenotype of animals within this condition approximated the findings in Anniston with respect to physical characteristics of metabolic syndrome and 
serological evidence of liver injury, inflammation, and metabolic dysfunction. The exposure to higher levels of PCBs in this animal model did not result in additional histological evidence of liver injury, suggesting that it was possible to observe nonmontonic dose responses to PCBs with respect to serological biomarkers of liver injury.

One of the major findings of the Wahlang et al. study was that Aroclor 1260 exposure contributed to liver injury not by exacerbating steatosis, but by increasing inflammation, particularly in the $\mathrm{HVvsH} 20$ model. Our original hypothesis was that, at higher levels of Aroclor 1260 exposure, the concentration of trace dioxin-like PCBs within the mixture would reach a "tipping point" that would quell immune response in our inflammation-primed HFD-fed mice. Previous reports suggested that the ED 50 for Aroclor 1260 immunotoxicity (in mice) was 360 $\mathrm{mg} / \mathrm{kg}^{190}$, still higher than our highest in vivo dose. We saw differences in the moderate vs. high dose with regards to transcriptional output of IL-6 $\rightarrow$ JAK $\rightarrow$ STAT- 3 signaling, which plays a role in chronic inflammation.

Inflammatory response is a process that is both context-modulated and dynamic, involving initiation and resolution processes that confound a simple understanding of "proinflammatory" and "anti-inflammatory" signaling molecules. IL-6, which is produced by many cell types, including hepatocytes and Kupffer cells (liver-resident macrophages), is often categorized as a "pro-inflammatory" cytokine, and was elevated in both the ACHS cohort and in the in vivo moderate dose + HFD. Certainly, IL-6 is elevated in inflammation and has down-stream effects that promote inflammation; on the other hand, IL-6 has distinctly "anti-inflammatory" effects in the liver as well, particularly to oppose exaggerated cytokine release from neighboring Kupffer cells.

Components of IL-6 signaling pathways were more enriched in the moderate PCB/HFD model (Figure 22). IL-6 signaling mediators down-stream of STAT-3 transcriptional regulation leading to cell differentiation pathways (cFos and cJun - 1.23-fold and 0.64-fold increased, respectively), were also affected with the high PCB exposures (cJun only: 0.80 -fold, $p=0.00005$ and 1.15-fold, $p=0.00005$ in the high PCB exposure with HFD and CD, respectively). Another group of STAT-3 targets with PCB-dependent transcriptional changes were the suppressors of cytokine signaling (SOCS2 and SOCS3 - 1.10-fold and 0.71-fold increased, respectively in the 


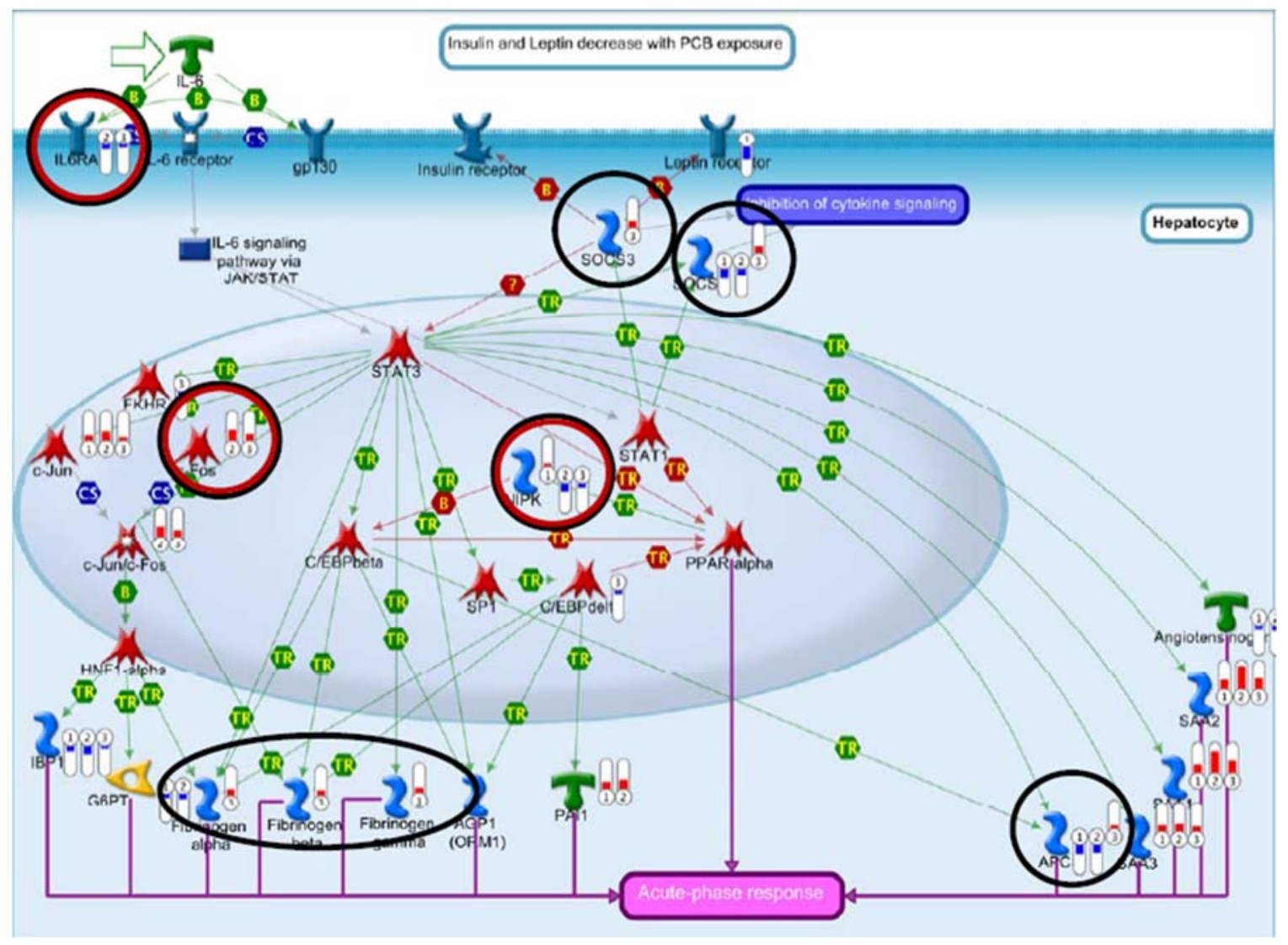

Figure 22. Exposure to PCB with or without HFD coexposure alters transcription of multiple components of IL-6 signaling. The red circles correspond to targets differentially regulated in a comparison between 0 and $20 \mathrm{mg} / \mathrm{kg}$ Aroclor 1260 within the control diet group while the black circles correspond to targets differentially regulated in a comparison between control and HFD within the Aroclor 1260 20mg/kg group. 
moderate PCB/HFD exposure). SOCS3 is an important player in the attenuation of IL-6-mediated STAT-3 signaling. Potently up-regulated by active STAT-3, SOCS3 binds to and inhibits JAK2/gp130 kinases, which couple IL-6 to STAT-3 signaling. SOCS3 also independently contributes to hepatic insulin resistance and metabolic dysfunction by binding to and inhibiting both insulin receptor and its down-stream mediator IRS-1 ${ }^{191}$ and well as leptin receptor ${ }^{192}$. SOCS3 was only up-regulated in the moderate PCB/HFD exposure, while in the high PCB/HFD exposure, SOCS2 was up-regulated as well $(0.71$-fold, $p=0.00005)$.

In addition to transcriptional changes down-stream of STAT-3 signaling, the HFD/moderate exposure animals had decreased transcription of IL-6 receptor alpha (IL-6Ra: 0.67-fold, $p=0.0000)$. IL-6Ra plays an important role in mechanisms contributing to chronic STAT-3 activation and related metabolic dysfunction and chronic inflammation. Of interest is the relationship between IL-6R $\alpha$ and EGFR. Independent of EGF activation, EGFR forms a complex with both IL6R $\alpha$ and STAT-3 in a mechanism which potentiates STAT-3 phosphorylation even in the presence of SOCS-3 $3^{193}$. EGFR/IL-6Ra-mediated STAT-3 activation contributes to chronic IL6 expression by hepatocytes ${ }^{193}$. Expression of EGFR itself was down-regulated in HFD alone (0.85 -fold, $p=0.0027$ ), but was up-regulated in both the moderate and high PCB + HFD exposures (1.15-fold, $p=0.00005$ and 0.78 -fold, $p=0.0011$, respectively). If protein levels were similarly affected, this might represent a mechanism by which PCBs and HFD contribute to chronic IL-6 release. Alternatively, liver-specific knockout of IL-6Ra has been shown to enhance the release of IL-6 and TNF $\alpha$ from Kupffer cells (liver-specific tissue-resident macrophages), preventing the IL-6-mediated down-regulation of these cytokines. Abrogation of this receptor in liver parenchymal cells also reduces insulin-stimulated glucose uptake in white adipose tissue and skeletal muscle, contributing to systemic insulin resistance ${ }^{194}$. Therefore, by altering IL-6 signaling within hepatocytes, PCBs may affect not only the transduction of inflammatory signals in the liver, but also signals coordinating metabolic response between tissues. Future studies may be able to pinpoint whether PCB-mediated EGFR/IL-6R $\alpha / S T A T-3$ perpetuation of STAT-3 signaling and/or IL-6Ra inhibition contribute to systemic IL-6 elevation, which has been associated with T2DM and many other metabolic diseases. 
Another factor contributing to increased systemic IL-6 may be hyaluronic acid, an extracellular matrix protein which was significantly increased with PCB exposure in sera from the ACHS-II cohort. Hyaluronic acid binds and stabilizes extracellular IL-6, resulting in increased serum protein levels without affecting IL-6 mRNA transcription ${ }^{195}$. Multiple factors contributing to high chronic systemic IL-6 may therefore represent a self-sustaining system by which PCBs exacerbate pre-existing metabolic dysfunction. Chronic IL-6 elevation may also represent a point of convergence for liver injury mechanisms, agreeing well with the "multiple-hits hypothesis".

PCB exposure did not contribute to increased steatosis in our animal model beyond the effects of HFD, nevertheless, genes encoding elements of hepatic lipid processing were differentially transcribed in the exposure models relative to vehicle-treated controls on the same diet. Key events regulating the development of steatosis include increased fatty acid uptake, increased lipid synthesis, decreased fatty acid oxidation, and decreased lipid efflux. ${ }^{56}$ Transcription of CD36, a FA uptake receptor, was up-regulated in high-exposure/control diet (0.74, $p=0.0004)$ and with HFD alone (CVvsHV: 0.57 -fold, $p=0.00185)$, and was down-regulated in both moderate exposure models (CVvsC20: -0.75 -fold, $\mathrm{p}=0.00045$, and $\mathrm{HVvsH20:}-0.89$-fold, $p=0.00005)$. Transcription of long-chain acyl-CoA synthetase 4 (ACSL4), involved in lipid synthesis, was down-regulated in high-exposure/control diet $(-0.47$-fold, $p=0.00315)$ as in HFD alone $(-0.91$-fold, $p=0.00005)$, but was up-regulated in moderate exposure/HFD (1.05-fold, $p=0.00005)$. These patterns do not suggest a consistent increase in pro-steatotic molecular changes, which would be consistent with the observation that Aroclor 1260 exposure (particularly at the moderate dose) did not worsen steatosis. They do, however, suggest that PCB exposure at both high and low levels affects cellular events which can alter hepatocellular lipid flux, and, moreover, PCB exposure affects the components of those events which are known to be influenced by nuclear receptor-driven transcriptional changes. ${ }^{56}$

The classically understood function of xenobiotic receptors is to respond to the presence of chemical ligands by heterodimerizing with RXR (nuclear receptors) or ARNT (AhR), translocating to the nucleus, and binding response elements in the promoters of their respective cytochrome p450 oxidase targets. A more nuanced understanding includes the association of 
unliganded receptors with specific binding partners in a corepressor complex and liganded receptors with coactivators. In addition to ligand-binding, post-translational modifications such as phosphorylation, acetylation, and sumoylation can change the receptor's protein-protein associations, adding tremendous range to both the direct and indirect transcriptional targets affected by xenobiotic sensing. This provides a compelling mechanism by which the changing chemical environment can shape the pattern of physiological metabolism.

Just as multiple systems create a dynamic balance by shifting chemical pressures, rhythmic metabolic processes affected by the central or peripheral circadian clock alter metabolic tides in response to temporal cues. An exploration of PCB effects on the suprachiasmatic nucleus (SCN) which regulates the central clock is beyond the scope of this work, however, the behavioral changes in our mouse model described in Chapter 2 (anachronistically increased movement during typical sleep cycles in transgenic mice chronically exposed to $20 \mathrm{mg} / \mathrm{kg}$ Aroclor 1260 ) are most likely a manifestation of central clock alterations. In peripheral tissues including the liver, however, oscillations in the transcription of specific gene sets create feedback loops which reinforce the periodic cycling within those tissues. Importantly, the challenge of nutritional stress HFD $^{136}$ or time-restricted ${ }^{135}$ feeding - can uncouple the peripheral circadian clock from the central, leading to differential temporal expression of targets of timekeeping transcription factors (CLOCK and BMAL1) as well as new oscillations of previously non-cycling gene targets of transcription factors such as PPARY ${ }^{136}$.

It is not now known whether the behavioral changes seen in our transgenic mouse model were a direct effect of PCBs on the SCN neurons or a product of peripheral PCB effect feeding back by some mechanism to reprogram the central clock. Studies investigating the effects of dietary stresses on circadian cycling have indicated that while peripheral tissues are reprogrammed by nutritional challenge, the SCN central pacemaker remains unaffected. However, these studies have also reported changes to the clock output genes (Per1/2 and Cry1/2 isoforms) without changes to expression of the core clock genes (Clock and Bmal1). In our in vivo experiment, we observed changes in both clock output and core clock genes under multiple conditions (Table 64). Differential transcription of Clock, however, was unique to the moderate 


\section{Table 64}

Differentially transcribed circadian-rhythm related targets from in vivo and in vitro experiments

\begin{tabular}{|c|c|c|c|c|c|c|c|}
\hline & \multicolumn{5}{|c|}{ In vivo } & \multirow{2}{*}{\multicolumn{2}{|c|}{$\begin{array}{c}\text { In vitro } \\
\text { FC vs. DMSO }\end{array}$}} \\
\hline & \multirow[t]{2}{*}{ HFD vs CD } & \multicolumn{2}{|c|}{ FC vs. CD/V } & \multicolumn{2}{|c|}{ FC vs. HFD/V } & & \\
\hline & & $\mathrm{C} 20$ & C200 & $\mathrm{H} 2 \mathrm{O}$ & $\mathrm{H} 200$ & $\mathrm{PCN}$ & $B A$ \\
\hline \multicolumn{8}{|c|}{ Core Clock Genes } \\
\hline Clock & & 0.77 & & & & & \\
\hline Bmal1 & & 2.19 & 1.04 & & & -0.81 & -0.73 \\
\hline \multicolumn{8}{|c|}{ Clock output Genes } \\
\hline Cry1 & -0.73 & & & & 0.65 & & \\
\hline Cry2 & & & 0.48 & & & -0.47 & \\
\hline Per1 & & & & -0.97 & & & \\
\hline Per2 & & -1.39 & -0.74 & & & & \\
\hline \multicolumn{8}{|c|}{ Selected Clock-associated genes } \\
\hline $\begin{array}{l}\text { Melatonin } \\
\text { receptor } \\
1 \mathrm{~A}\end{array}$ & 2.80 & & 1.51 & & & & \\
\hline ROR-g & & -0.74 & 0.88 & & 0.53 & & \\
\hline TrkB & 0.66 & -1.28 & -2.03 & & 0.72 & & \\
\hline
\end{tabular}

Table 64 shows fold changes for the comparisons listed above. Targets are grouped by their relationship to circadian rhythm control machinery (core clock genes, clock output genes, or clock-associated genes). Numbers are fold change vs. the indicated control, $p<.05$. Blank fields were not significantly different in the listed comparison. 
$\mathrm{PCB} /$ control diet condition, however, where it was up-regulated compared to vehicle/control diet $(0.77$-fold, $p=0.00005)$. Our in vitro experiment did not indicate differential regulation of clockrelated genes with Aroclor 1260 exposure, but did indicate down-regulation of one core clock gene (Bmal1) with PCN or BA, and down-regulation of one clock output gene (Cry2) with PCN exposure. The direction of fold change was again opposite from any of the in vivo conditions in which these genes were differentially transcribed.

The mechanism by which PCBs affect the transcription of peripheral circadian rhythm machinery is unclear from our in vivo or in vitro experiments, although there exists no shortage of potential interactions between elements of these processes and xenobiotic receptors. BMALI1, also known as the arylhydrocarbon receptor nuclear translocator-like (ARNTL), and CLOCK are PAS-domain proteins which readily associate with AhR, and their regulation of Per1 has been shown to be disrupted by AhR activity in response to TCDD ${ }^{196}$. In addition, the hepatocytespecific cyclic AMP responsive element-binding protein (CREBH) is a transcription factor with transcriptional activity controlling the rhythmic expression of hepatic triglyceride and fatty acid metabolic targets via its associations with PPARa and $\mathrm{LXRa}^{131}$, which crosstalk with xenobiotic nuclear receptors as previously described. The processes leading to circadian activation of CREBH are controlled by BMAL1 via GSK3ß/AKT pathways ${ }^{131}$. Other isoforms of CREB physically interact with and are inhibited by PXR (Figure 13). ${ }^{197}$

A clear limitation of the experiment in this area is the lack of tissue harvest timepoints designed to capture oscillations in transcription at multiple timepoints. Harvest was carried out only during the light cycle and over a period of approximately 8 hours. Future studies to explore PCB effect in this area could be modeled on the methods of Eckel-Mahan et al. ${ }^{136}$, to capture potential PCB exposure-related shifts in the temporal expression of normally cyclic or normally acyclic hepatic genes.

A final pattern observed in the enrichment analyses of both the in vivo and in vitro study was that of cell adhesion and related cell-cell communications apparatus. Components of ephrin signaling were highly differentially expressed between conditions. Ephrin receptors are part of a large class of receptor tyrosine kinases which form bi-directional communication complexes 
between cells. Ephrins and ephrin receptors are well-studied in the context of cytoskeletal remodeling during neuronal path-finding and development, and, importantly, play a role in adhesion and communication between cells of the same or different types, including monocytes and endothelial cells ${ }^{198}$. The ephrin signaling system consists of two classes of receptor ( $\mathrm{A}$ and $\mathrm{B}$, based on sequence homology and corresponding to structural characteristics) which respond to ligands produced by neighboring cells ${ }^{199}$. Both receptor and ligand are generally membranebound, facilitating communication between cells that are in physical contact, however, there is evidence that some ligands, specifically Ephrin A1, can be shed as soluble ligands to affect cells over longer distances ${ }^{200}$. In the ephrin signaling system, signal transduction in the direction of the receptor is described as forward-signaling and signal transduction in the direction of the ligand is described as reverse signaling. Ephrin-A/EphA forward signaling affects many of the pathways activated by other RTKs, including IGF-1-activated Ras-Erk, and may inhibit or activate these cascades in a cell-type specific manner ${ }^{201}$.

In our experimental models, both ephrin receptors and ephrin ligands were differentially regulated under some or all conditions (Figures 23-25). Interestingly, Ephrin A1 (Efna1) was significantly up-regulated in every in vivo comparison except moderate PCB/control diet exposure as well as in vitro PCB exposure, and was one of the targets which was uniquely regulated in PCB exposure (Figure 23). Ephrin A5 (Efna5), another ligand, was also up-regulated in all HFDexposed groups and and upon exposure to $200 \mathrm{mg} / \mathrm{kg}$ PCB with CD (Figure 24). Ephrin A3 (Efna3) was strongly up-regulated only in the moderate PCB/HFD coexposure (3.85-fold, $p=0.00095)$, and was otherwise not significantly different from vehicle/CD. Ephrin receptor B2 was differentially regulated under all conditions (Figure 25).

Differential expression of ephrin receptors and ligands is associated with proinflammatory states and changes in inflammatory cytokines in multiple tissue. Expression of EphA2 (receptor) and Ephrin A1 (ligand) have been shown to increase in lung tissue after LPS exposure, and posttreatment with mAb against EphA2 receptor reduces lung injury and permeability in LPS-exposed animals, as well as the production of pro-inflammatory cytokines $(I L 1 \beta, M I P-2)^{202}$. 


\section{A. $\quad$ Ephrin A1 ligand (Efna1) Expression (in vivo)}

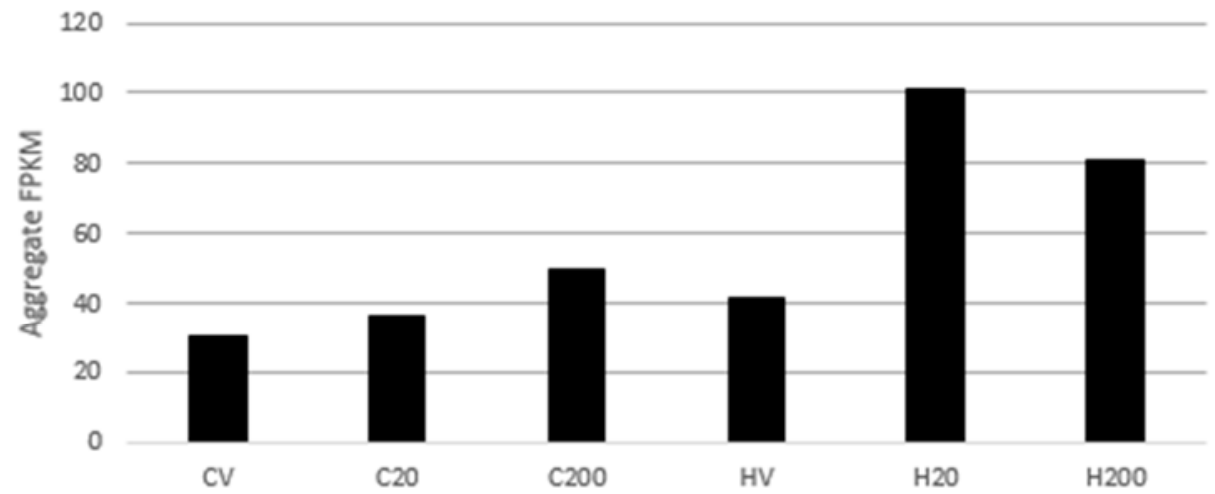

B. Ephrin A1 ligand (Efna1) Expression ( $1^{\circ}$ Hepatocytes)

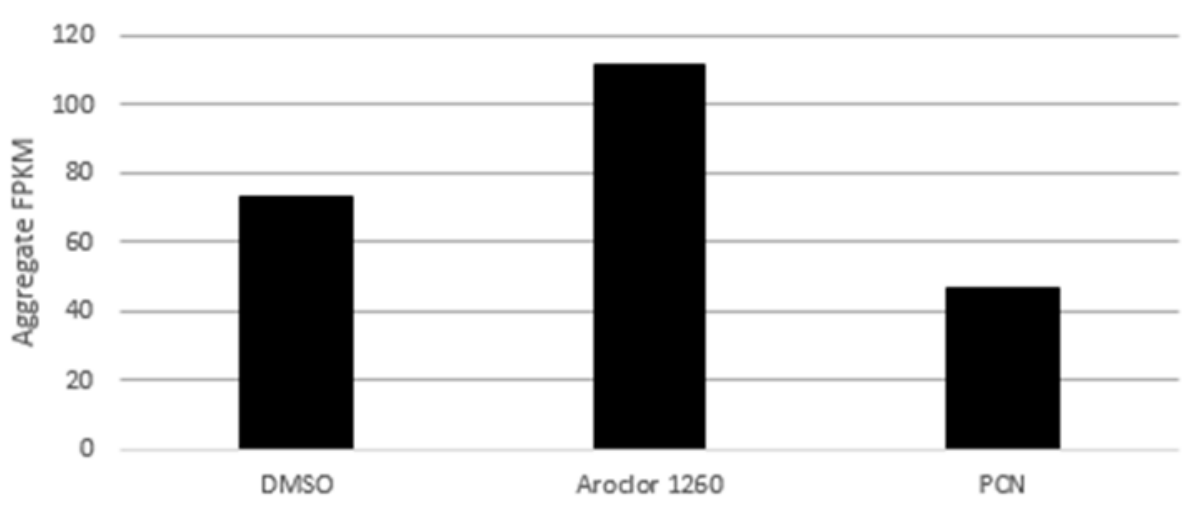

Figure 23. Ephrin ligand A1 (Efna1) was differentially regulated in multiple comparisons. The vertical axis shows aggregate fragments per kilobase of transcript per million mapped reads (FPKM) for each condition, with significant differences indicated by bridging lines. Panel A shows the respective aggregate FPKM for six in vivo conditions, while Panel B shows the same for the in vitro experiment, with exposure to Aroclor 1260 and PCN shown. Treatment with prototypical ligands for AhR, CAR, and LXR did not induce significant changes in Efna1 expression. 


\section{Ephrin A5 ligand (Efna5) Expression}

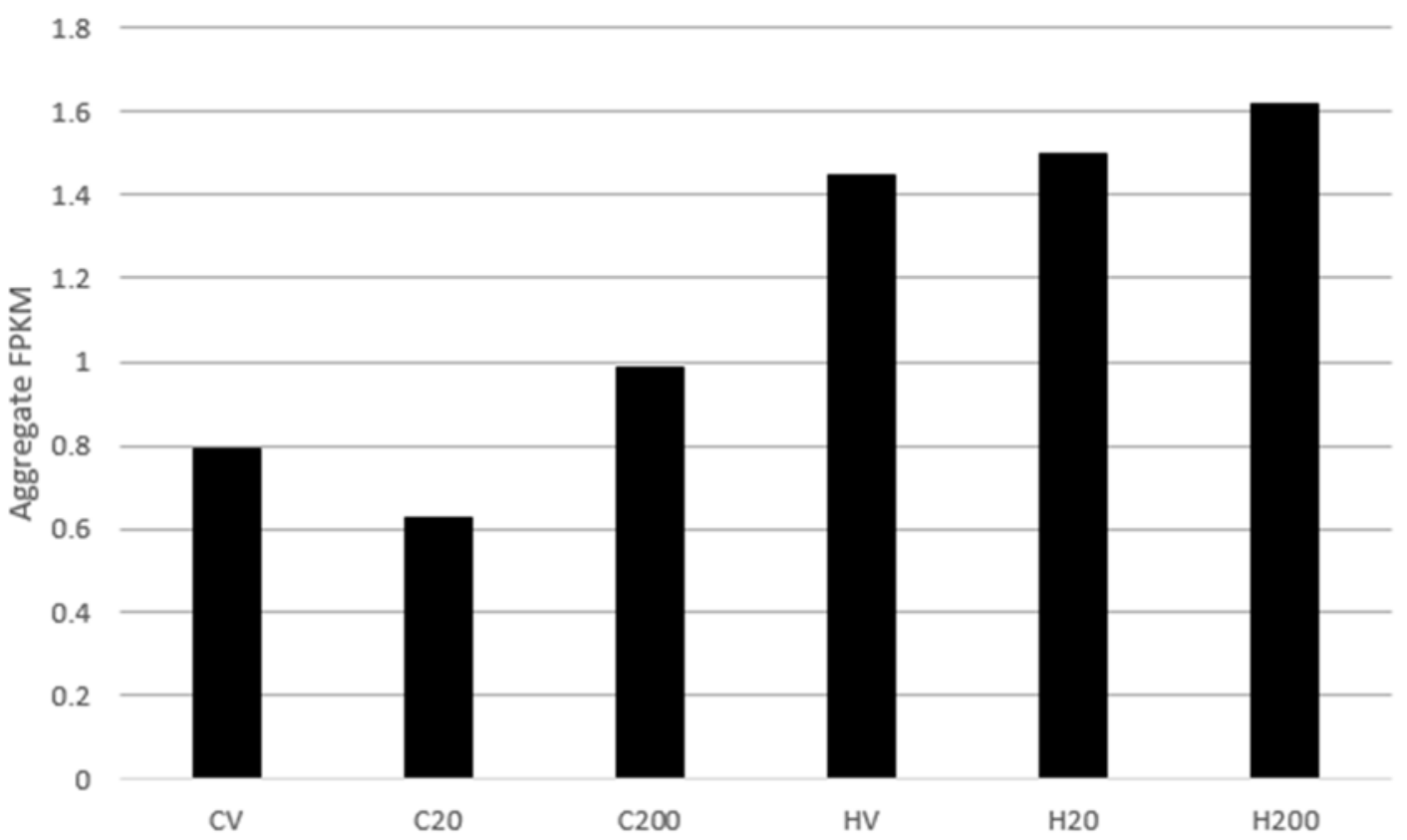

Figure 24. Ephrin ligand A5 (Efna5) was differentially regulated in multiple comparisons. The vertical axis shows aggregate fragments per kilobase of transcript per million mapped reads (FPKM) for each condition, with significant differences indicated by bridging lines. 


\section{Ephrin-B Receptor 2 (Ephb2) Expression}

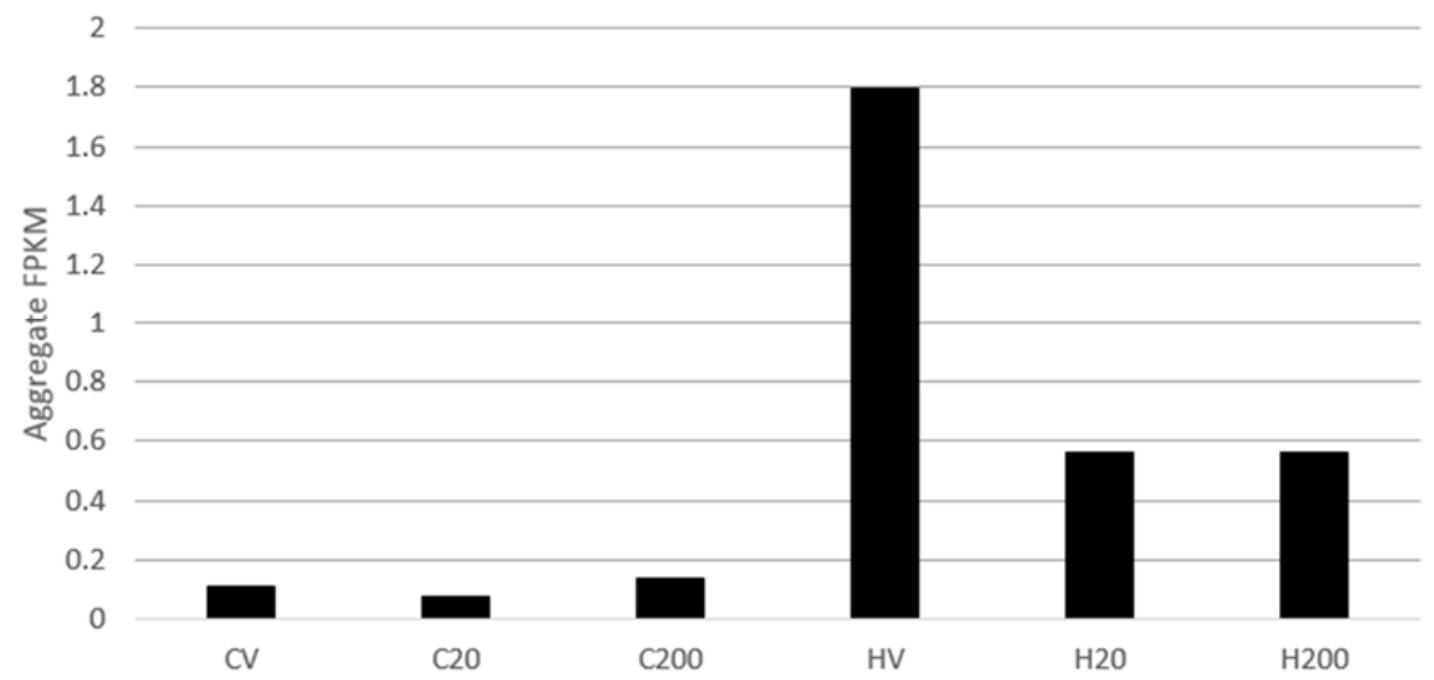

Figure 25. Ephrin receptor B2 (EphB2) was differentially regulated in multiple comparisons. The vertical axis shows aggregate fragments per kilobase of transcript per million mapped reads (FPKM) for each condition, with significant differences indicated by bridging lines. 
Differential expression of ephrin receptors and ligands is also associated with transitions between differentiated (epithelial) and undifferentiated (mesenchymal) cell characteristics. In the context of cancer, it has been observed that overexpression of ephrin receptors tends to favor metastatic characteristics (loss of adhesion), while overexpression of ligand tends to favor increased cell adhesion ${ }^{203}$. To that effect, EphA2 was found to be overexpressed in neoplastic cells, and the distribution changed from clustered localization of EphA2 at sites of cell-cell contacts to either a diffuse pattern or concentration in the leading edge of migrating cells ${ }^{204}$. This loss of localization at sites of stable cell-cell contacts led to decreased ligand-dependent phosphorylation of EphA2, because the membrane-bound Ephrin-A ligands also localize at these sites. E-cadherin forms homodimers with E-cadherin in adjacent cells to mediate highly stable calcium-dependent cell adhesion foci, and E-cadherin function in these cell-cell junctions is necessary to prevent decreased phosphorylation of EphA2. Given the role of ephrin receptors and ligands in cytoskeletal remodeling during growth and development as well as regeneration, development, especially with the sequential events necessary for cells to form properly oriented relationships with one another. In all PCB exposures, both in vivo and in vitro, the expression of adhesion proteins such as Claudin-1 and E-Cadherin was up-regulated vs. untreated control. Most literature describing changes to these proteins in the context of liver disease deals with their importance as biomarkers of cell identity during periods of physiological or pathological change: development, regeneration, and neoplasia. Up-regulation of these proteins is associated with a more terminally differentiated, epithelioid cell, but in the larger context, expression of these proteins is necessary for proper orientation of cells relative to one another within the larger tissue structure.

Ephrin signaling is important in other tissues which participate in metabolic allostasis. For instance, pancreatic beta cells employ ephrin signaling to synchronize release of insulin in response to glucose, using engagement of EphA forward signaling to inhibit insulin secretion and ephrin-A reverse signaling to stimulate insulin secretion ${ }^{205}$. If expression of Ephrin-A1 is similarly up-regulated in the pancreas as it is in the liver, this may represent another mechanism by which 
PCBs and PCB+HFD contribute to the phenotype of reduced serum insulin observed in both Anniston and our mouse model.

Xenobiotic receptors are known to crosstalk with signaling cascades down-stream of tyrosine kinase signaling. Ephrin receptor signaling may crosstalk with regulation of the constitutive androstane receptor in a similar way to EGFR. The receptor for activated c-kinase 1 (RACK-1) associates with ligand-bound, autophosphorylated and active EphB3, forming a complex with PP2A and AKT and promoting the dephosphorylation of AKT at S473 ${ }^{203}$. an event which leads to reduced cell migration as well as apoptosis. Active (dephosphorylated) RACK-1 also facilitates the interaction of CAR with PP2A, an event which is necessary for the nuclear translocation of CAR, the indirect or ligand-independent pathway for CAR activation described by Mutoh et al. ${ }^{21}$ This pathway is critical to PCB research, because it activates CAR via EGFR inhibition, a mechanism which is shared by phenobarbital and PCB congeners ${ }^{20}$. Input into CAR transcriptional activity via ephrin signaling represents a mechanism by which inflammatory status and cell-cell communication status can crosstalk with the output of xenobiotic receptors, providing more nuanced control over adaptive metabolic control. Our in vitro experiment, however, indicated that only one target related to ephrin signaling was differentially regulated by Aroclor 1260 exposure: the Ephrin A ligand Efna1 (Figure 26, Panel B). Interestingly, Efna1 was also differentially regulated in hepatocytes cells exposed to PCN, although in an opposite direction (0.66-fold, $p=0.00005$ ), which may be another example of the trend of PCB-dependent upregulation of targets in vivo which are negatively regulated by PCN exposure, and presumably PXR activation, in vitro.

Our combined approach of epidemiological assessment followed by in vivo and in vitro mechanistic exploration has uncovered many interesting relationships between environmental PCB exposure and human disease. While the question of the roles of xenobiotic receptors in PCB-associated fatty liver disease is far from resolved, we have at least pushed the understanding of those roles beyond direct, ligand-activated transcriptional activity of a few (or worse, one), xenobiotic-responsive transcription factor. It is likely that a great many of the effects of PCB-associated TASH are independent of direct effects of CAR, PXR, or AhR. It is equally 
likely that input from these receptors is indirectly involved in the pathways and processes modulated by PCB effects. We have described three groups of effects which are related to effects seen in all three of our models: systemic inflammatory dysregulation via IL-6 signalling, disruption of circadian rhythm processes, and modulation of cell adhesion and cell-cell communication via differential expression of E-cadherin/claudin-1 and related ephrin signaling pathways. These effects appear to be directly related to PCB exposure, are strongly involved in and modulated by dietary coexposures, and are likely to affect multiple systems involved in metabolic disease. Simultaneous dysregulation of these systems during PCB exposure could explain the "2-hit" phenomenon, providing a mechanism by which PCB/HFD coexposures could accelerate allostatic overload - crippling the normal adaptive response to metabolic challenge.

Future studies will likely focus on individual components of these effects, and may involve analysis of tissues other than liver. It remains to be determined whether PCB-dependent changes to IL-6 signaling lead to failure of feedback inhibition from hepatocytes to Kupffer cells and subsequent uncontrolled inflammation, or whether the systemic IL-6 elevations seen in Anniston arise from a different tissue - adipose, for instance. Likewise, an assessment of the PCBdependent changes to circadian signaling require evaluation of PCB-dependent changes to clock output and, more intriguingly, core clock gene transcription not only in peripheral tissues, but in the SCN as well. Exploring the mechanism and effects of the PCB-dependent transcriptional changes to ephrin signaling components and cell adhesion molecules may allow us to determine whether cell-cell communication between hepatocytes, endothelial cells, and/or Kupffer cells are dysregulated in PCB exposure, and whether changes to cell adhesion mediators is indicative of failed regenerative processes which could explain the apparent "chronic necrosis" observed in the Anniston population as well as the increased biomarkers of fibrosis.

As for the activities of the xenobiotic receptors themselves, our in vitro model was interesting from the standpoint that it showed that a substantial portion of the targets differentially transcribed with Aroclor 1260 exposure were not attributable to activation of AhR, PXR, CAR, or LXR. Targets within the set differentially transcribed with Aroclor 1260 exposure were associated with many liver and metabolic disease states, however, in a direct comparison between targets 
differentially transcribed with Aroclor 1260 exposure in vitro and in vivo, there were very few shared between the in vitro (Figure 27) and moderate exposure, with far more shared between the in vitro and high exposure. Although the in vitro experiment used $5 \mu \mathrm{g} / \mathrm{ml}$ Aroclor 1260 , a concentration lower than the concentration used to induce activity of human PXR, CAR, and AhR transfected into a HepG2 model luciferase reporter system ${ }^{19}$, there were more DTGs shared between the in vitro and the two high-dose in vivo models than the in vitro and two moderatedose in vivo models. It is possible, therefore, that $5 \mu \mathrm{g} / \mathrm{ml}$ is still too high to accurately model a relevant environmental exposure in primary hepatocytes. With that in mind, however, targets such as Claudin-1, E-Cadherin, Ephrin-A, and Tribbles-3 pseudokinase were conserved across more than one of the comparisons, suggesting that they may be part of common mechanisms for PCB effect. Because the raw data from the in vitro work will be available for further interrogation, it is likely that alternative bioinformatics tools may bring some clarity to the specific roles of these receptors in the observed transcriptional changes. If, instead of direct transactivation PXR, CAR, and/or AhR are involved via protein-protein interactions with other transcription factors, this may be due to a PCB-dependent intracellular milieu that favors specific post-translational modifications and corresponding specific protein associations.

This laboratory set out to investigate the relationship between exposure to PCBs a widespread class of persistent organic pollutant, and steatohepatitis, a widespread manifestation of metabolic disease. Because xenobiotic receptors drive the metabolic machinery responsible for recognizing and detoxifying foreign chemicals, and because of historic attention to the role of one part of this machinery, the cytochrome p450s, in generating carcinogenic metabolites from polycyclic aromatic hydrocarbons ${ }^{206}$ including PCBs, we and others expected to find a central role for these receptors which placed down-stream transcriptional targets as effectors in disease. Although some targets of these receptors are undoubtedly involved in steatohepatitis and other disease states, I find that the evidence from this study suggests a more peripheral role for these xenobiotic receptors in PCB-associated TASH: modulating metabolic responses with the additional information about the presence of toxicants. This makes sense from a standpoint of phenotype: moderate-dose PCBs alone did not induce steatohepatitis in our mouse model, and 

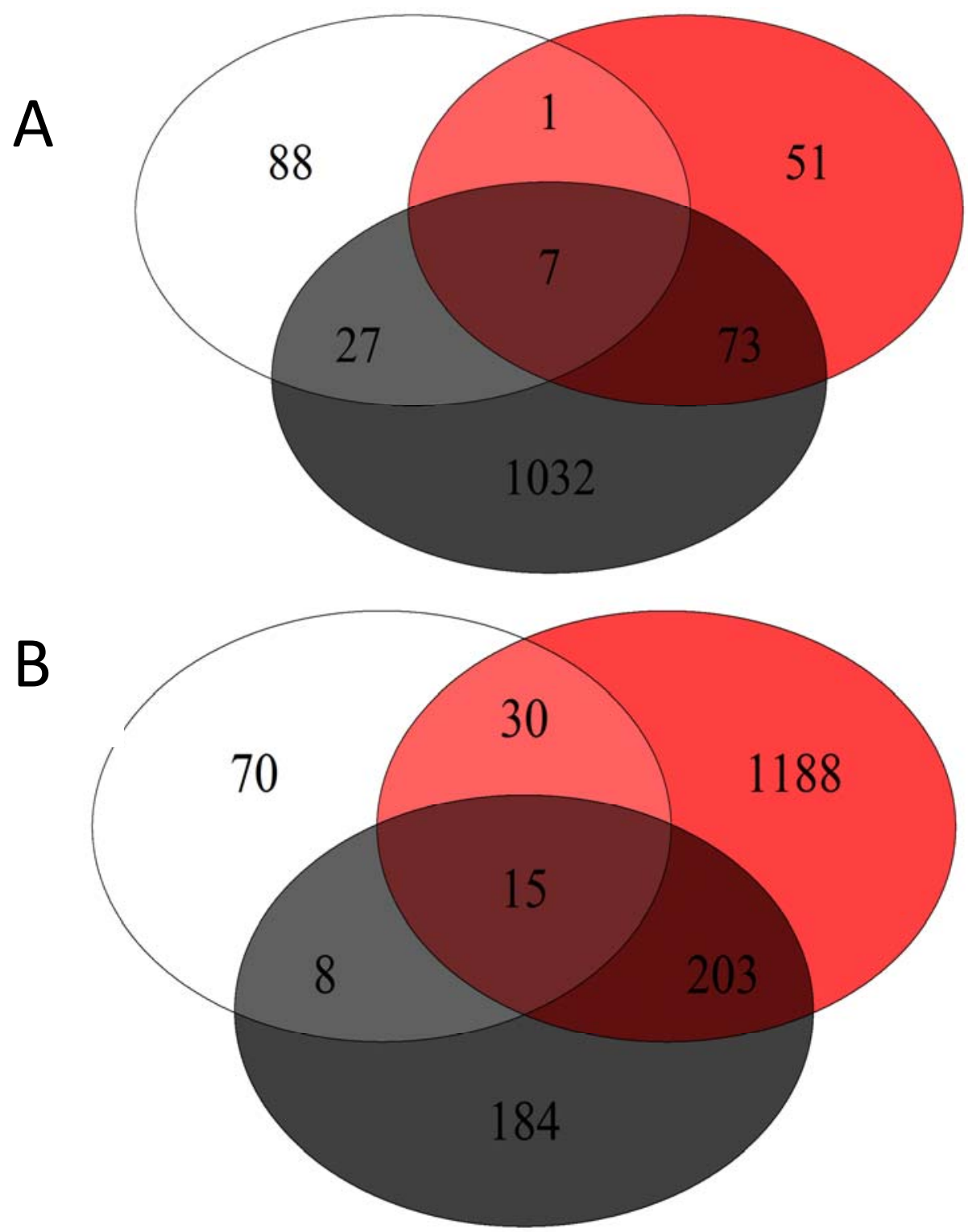

Figure 26. In vivo comparison diagrams. Venn diagrams show the overlapping region between in vitro study (white), $20 \mathrm{mg} / \mathrm{kg}$ Aroclor 1260 in vivo (red) and $200 \mathrm{mg} / \mathrm{kg}$ in vivo (black) on in vivo datasets grouped by diet. Panel A, control diet, shows a larger proportion of DTGs overlapping between the in vivo and high Aroclor. Shared targets include Gstm3, Cldn1, Pim1, Orm3, Trib3, and Akr1b7. In Panel B, which shows the overlap with the HFD in vivo expsoures, a larger proportion of the DTGs identified in the in vitro Aroclor 1260 exposure overlapped with the moderate in vivo exposure + high fat diet. Shared targets include Cdh1, Moxd1, Slc10a2, Efna1, Orm3, Rab30, Cyp1a2, Hist1h1c, Rarres1, Ces2b, Akr1b7, Ugt2b37, Orm2, and G6pc. 
there is not a clear dose-response relationship between total PCB load or the concentration of a defined PCB functional grouping and TASH in Anniston. The requirement for co-occurring metabolic stress would also explain the convergence in phenotype seen in both human NASH/TASH and in mouse models. PCB exposure appears to change elements of the response to this metabolic pressure - hepatocyte death mechanism, and inflammatory response, for instance, or elements of cell-cell signaling and adhesion important in regeneration. Lastly, the extrahepatic effects of PCBs on the liver cannot be overemphasized. Our human population showed inappropriate insulin production in response to hyperglycemia, strongly suggesting direct PCB effects in the pancreas, and a transgenic mouse (CAR-/-) in our laboratory demonstrated altered circadian patterns in response to PCB. One or both of these effects may involve the activity of xenobiotic receptors - their role in mediation of PCB effect in extrahepatic tissues is beyond the scope of this project - however, both effects have the potential to impact the development of steatohepatitis through effects on other metabolic allostasis machinery. These studies have strengthened the body of evidence connecting environmental PCB exposures to toxicant-associated steatohepatitis, have shifted the focus of mechanism from strictly xenobioticreceptor-mediated effects, and have pointed to new areas of inquiry we can explore to address the health effects of PCB exposure in the Anniston population and in all PCB-exposed humans. 


\section{REFERENCES}

[1] A.T.S.D.R. (2015) The Priority List of Hazardous Substances That Will Be the Candidates for Toxicological Profiles.

[2] US Environmental Protection Agency (1979) Polychlorinated Biphenyls 1929-1979: Final Report National Technical Information Service, Springfield, VA.

[3] Erickson, M. D. (2015) Introduction: PCB properties, uses, occurance and regulatory history, In PCBs : recent advances in environmental toxicology and health effects (Robertson, $L$. W., and Hansen, L. G., Eds.), pp xi-xxx, The University Press of Kentucky, Lexington :.

[4] Saba, T., and Boehm, P. D. (2011) Congener-based analysis of the weathering of PCB Aroclor 1242 in paper mill sludge, Chemosphere 82, 1321-1328.

[5] Metcalfe, T. L., and Metcalfe, C. D. (1997) The trophodynamics of PCBs, including mono- and non-ortho congeners, in the food web of North-Central Lake Ontario, Sci Total Environ 201, 245-272.

[6] Drinker, C. K., Warren, M. F., and Bennett, G. A. (1937) The Problem of Possible Systemic Effects from Certain Chlorinated Hydrocarbons, Journal of Industrial Hygiene and Toxicology 19, 283-299.

[7] Maroni, M., Colombi, A., Arbosti, G., Cantoni, S., and Foa, V. (1981) Occupational exposure to polychlorinated biphenyls in electrical workers. II. Health effects, $\mathrm{Br} J$ Ind Med 38, 55-60.

[8] Smith, A. B., Schloemer, J., Lowry, L. K., Smallwood, A. W., Ligo, R. N., Tanaka, S., Stringer, W., Jones, M., Hervin, R., and Glueck, C. J. (1982) Metabolic and health consequences of occupational exposure to polychlorinated biphenyls, Br J Ind Med 39, 361-369.

[9] Fischbein, A. (1985) Liver function tests in workers with occupational exposure to polychlorinated biphenyls (PCBs): comparison with yusho and yu-cheng, Environ Health Perspect 60, 145-150.

[10] Lawton, R. W., Ross, M. R., Feingold, J., and Brown, J. F., Jr. (1985) Effects of PCB exposure on biochemical and hematological findings in capacitor workers, Environ Health Perspect 60, 165-184.

[11] Prince, M. M., Hein, M. J., Ruder, A. M., Waters, M. A., Laber, P. A., and Whelan, E. A. (2006) Update: cohort mortality study of workers highly exposed to polychlorinated biphenyls (PCBs) during the manufacture of electrical capacitors, 1940-1998, Environ Health 5, 13.

[12] Schecter, A., Stanley, J., Boggess, K., Masuda, Y., Mes, J., Wolff, M., Furst, P., Furst, C., Wilson-Yang, K., and Chisholm, B. (1994) Polychlorinated biphenyl levels in the tissues of exposed and nonexposed humans, Environ Health Perspect 102 Suppl 1, 149-158.

[13] Wolff, M. S., and Schecter, A. (1992) Use of PCB blood levels to assess potential exposure following an electrical transformer explosion, J Occup Med 34, 1079-1083.

[14] Masuda, Y., Kuroki, H., Haraguchi, K., and Nagayama, J. (1985) PCB and PCDF congeners in the blood and tissues of yusho and yu-cheng patients, Environ Health Perspect 59, 5358.

[15] Ikeda, M. (1996) Comparison of clinical picture between Yusho/Yucheng cases and occupational PCB poisoning cases, Chemosphere 32, 559-566.

[16] Safe, S. H. (1994) Polychlorinated biphenyls (PCBs): environmental impact, biochemical and toxic responses, and implications for risk assessment, Crit Rev Toxicol 24, 87-149.

[17] Sorg, O. (2014) AhR signalling and dioxin toxicity, Toxicol Lett 230, 225-233.

[18] Patterson, A. T., Kaffenberger, B. H., Keller, R. A., and Elston, D. M. (2016) Skin diseases associated with Agent Orange and other organochlorine exposures, J Am Acad Dermatol 74, 143-170. 
[19] Wahlang, B., Falkner, K. C., Clair, H. B., Al-Eryani, L., Prough, R. A., States, J. C., Coslo, D. M., Omiecinski, C. J., and Cave, M. C. (2014) Human receptor activation by aroclor 1260, a polychlorinated biphenyl mixture, Toxicol Sci 140, 283-297.

[20] Hardesty, J. E., Wahlang, B., Falkner, K. C., Clair, H. B., Clark, B. J., Ceresa, B. P., Prough, R. A., and Cave, M. C. (2017) Polychlorinated biphenyls disrupt hepatic epidermal growth factor receptor signaling, Xenobiotica 47, 807-820.

[21] Mutoh, S., Sobhany, M., Moore, R., Perera, L., Pedersen, L., Sueyoshi, T., and Negishi, M. (2013) Phenobarbital indirectly activates the constitutive active androstane receptor (CAR) by inhibition of epidermal growth factor receptor signaling, Sci Signal 6, ra31.

[22] Wahlang, B., Falkner, K. C., Gregory, B., Ansert, D., Young, D., Conklin, D. J., Bhatnagar, A., McClain, C. J., and Cave, M. (2013) Polychlorinated biphenyl 153 is a diet-dependent obesogen that worsens nonalcoholic fatty liver disease in male C57BL6/J mice, J Nutr Biochem 24, 1587-1595.

[23] Wahlang, B., Song, M., Beier, J. I., Cameron Falkner, K., Al-Eryani, L., Clair, H. B., Prough, R. A., Osborne, T. S., Malarkey, D. E., Christopher States, J., and Cave, M. C. (2014) Evaluation of Aroclor 1260 exposure in a mouse model of diet-induced obesity and nonalcoholic fatty liver disease, Toxicol Appl Pharmacol 279, 380-390.

[24] Wong, P. W., and Pessah, I. N. (1996) Ortho-substituted polychlorinated biphenyls alter calcium regulation by a ryanodine receptor-mediated mechanism: structural specificity toward skeletal- and cardiac-type microsomal calcium release channels, Mol Pharmacol 49, 740-751.

[25] Pessah, I. N., Hansen, L. G., Albertson, T. E., Garner, C. E., Ta, T. A., Do, Z., Kim, K. H., and Wong, P. W. (2006) Structure-activity relationship for noncoplanar polychlorinated biphenyl congeners toward the ryanodine receptor-Ca2+ channel complex type 1 (RyR1), Chem Res Toxicol 19, 92-101.

[26] Pessah, I. N., Cherednichenko, G., and Lein, P. J. (2010) Minding the calcium store: Ryanodine receptor activation as a convergent mechanism of PCB toxicity, Pharmacology \& therapeutics 125, 260-285.

[27] Abdelrahim, M., Ariazi, E., Kim, K., Khan, S., Barhoumi, R., Burghardt, R., Liu, S., Hill, D., Finnell, R., Wlodarczyk, B., Jordan, V. C., and Safe, S. (2006) 3-Methylcholanthrene and other aryl hydrocarbon receptor agonists directly activate estrogen receptor alpha, Cancer Res 66, 2459-2467.

[28] Zhang, Q., Lu, M., Wang, C., Du, J., Zhou, P., and Zhao, M. (2014) Characterization of estrogen receptor alpha activities in polychlorinated biphenyls by in vitro dual-luciferase reporter gene assay, Environ Pollut 189, 169-175.

[29] Bonefeld-Jorgensen, E. C., Andersen, H. R., Rasmussen, T. H., and Vinggaard, A. M. (2001) Effect of highly bioaccumulated polychlorinated biphenyl congeners on estrogen and androgen receptor activity, Toxicology 158, 141-153.

[30] Schrader, T. J., and Cooke, G. M. (2003) Effects of Aroclors and individual PCB congeners on activation of the human androgen receptor in vitro, Reprod Toxicol 17, 15-23.

[31] Van den Berg, M., Birnbaum, L. S., Denison, M., De Vito, M., Farland, W., Feeley, M., Fiedler, H., Hakansson, H., Hanberg, A., Haws, L., Rose, M., Safe, S., Schrenk, D., Tohyama, C., Tritscher, A., Tuomisto, J., Tysklind, M., Walker, N., and Peterson, R. E. (2006) The 2005 World Health Organization reevaluation of human and Mammalian toxic equivalency factors for dioxins and dioxin-like compounds, Toxicol Sci 93, 223-241.

[32] Rushneck, D. R., Beliveau, A., Fowler, B., Hamilton, C., Hoover, D., Kaye, K., Berg, M., Smith, T., Telliard, W. A., Roman, H., Ruder, E., and Ryan, L. (2004) Concentrations of dioxin-like 
PCB congeners in unweathered Aroclors by HRGC/HRMS using EPA Method 1668A, Chemosphere 54, 79-87.

[33] Jacobs, M. N., Nolan, G. T., and Hood, S. R. (2005) Lignans, bacteriocides and organochlorine compounds activate the human pregnane $\mathrm{X}$ receptor (PXR), Toxicol Appl Pharmacol 209, 123-133.

[34] Al-Salman, F., and Plant, N. (2012) Non-coplanar polychlorinated biphenyls (PCBs) are direct agonists for the human pregnane- $X$ receptor and constitutive androstane receptor, and activate target gene expression in a tissue-specific manner, Toxicol Appl Pharmacol 263, 7-13.

[35] Gahrs, M., Roos, R., Andersson, P. L., and Schrenk, D. (2013) Role of the nuclear xenobiotic receptors CAR and PXR in induction of cytochromes $\mathrm{P} 450$ by non-dioxinlike polychlorinated biphenyls in cultured rat hepatocytes, Toxicol Appl Pharmacol 272, 7785.

[36] Nault, R., Forgacs, A. L., Dere, E., and Zacharewski, T. R. (2013) Comparisons of differential gene expression elicited by TCDD, PCB126, betaNF, or ICZ in mouse hepatoma Hepa1c1c7 cells and C57BL/6 mouse liver, Toxicol Lett 223, 52-59.

[37] Cave, M., Appana, S., Patel, M., Falkner, K. C., McClain, C. J., and Brock, G. (2010) Polychlorinated biphenyls, lead, and mercury are associated with liver disease in American adults: NHANES 2003-2004, Environ Health Perspect 118, 1735-1742.

[38] Tee, P. G., Sweeney, A. M., Symanski, E., Gardiner, J. C., Gasior, D. M., and Schantz, S. L. (2003) A longitudinal examination of factors related to changes in serum polychlorinated biphenyl levels, Environ Health Perspect 111, 702-707.

[39] Hansen, L. G. (2013) The Ortho Side of Pcbs Occurrence and Disposition, Springer Verlag. [40] Virtue, S., and Vidal-Puig, A. (2010) Adipose tissue expandability, lipotoxicity and the Metabolic Syndrome--an allostatic perspective, Biochim Biophys Acta 1801, 338-349.

[41] Yang, Z. H., Miyahara, H., Takeo, J., and Katayama, M. (2012) Diet high in fat and sucrose induces rapid onset of obesity-related metabolic syndrome partly through rapid response of genes involved in lipogenesis, insulin signalling and inflammation in mice, Diabetol Metab Syndr 4, 32.

[42] Aydin, S., Aksoy, A., Aydin, S., Kalayci, M., Yilmaz, M., Kuloglu, T., Citil, C., and Catak, Z. (2014) Today's and yesterday's of pathophysiology: biochemistry of metabolic syndrome and animal models, Nutrition 30, 1-9.

[43] Lee, J. S., Jun, D. W., Kim, E. K., Jeon, H. J., Nam, H. H., and Saeed, W. K. (2015) Histologic and Metabolic Derangement in High-Fat, High-Fructose, and Combination Diet Animal Models, ScientificWorldJournal 2015, 306326.

[44] Della Vedova, M. C., Munoz, M. D., Santillan, L. D., Plateo-Pignatari, M. G., Germano, M. J., Rinaldi Tosi, M. E., Garcia, S., Gomez, N. N., Fornes, M. W., Gomez Mejiba, S. E., and Ramirez, D. C. (2016) A Mouse Model of Diet-Induced Obesity Resembling Most Features of Human Metabolic Syndrome, Nutr Metab Insights 9, 93-102.

[45] Chen, L. Z., Xin, Y. N., Geng, N., Jiang, M., Zhang, D. D., and Xuan, S. Y. (2015) PNPLA3 I148M variant in nonalcoholic fatty liver disease: demographic and ethnic characteristics and the role of the variant in nonalcoholic fatty liver fibrosis, World J Gastroenterol 21, 794802.

[46] Prudente, S., Hribal, M. L., Flex, E., Turchi, F., Morini, E., De Cosmo, S., Bacci, S., Tassi, V., Cardellini, M., Lauro, R., Sesti, G., Dallapiccola, B., and Trischitta, V. (2005) The functional Q84R polymorphism of mammalian Tribbles homolog TRB3 is associated with insulin resistance and related cardiovascular risk in Caucasians from Italy, Diabetes 54, 2807-2811. 
[47] Shi, Z., Liu, J., Guo, Q., Ma, X., Shen, L., Xu, S., Gao, H., Yuan, X., and Zhang, J. (2009) Association of TRB3 gene Q84R polymorphism with type 2 diabetes mellitus in Chinese population, Endocrine 35, 414-419.

[48] Barroso, I., Gurnell, M., Crowley, V. E., Agostini, M., Schwabe, J. W., Soos, M. A., Maslen, G. L., Williams, T. D., Lewis, H., Schafer, A. J., Chatterjee, V. K., and O'Rahilly, S. (1999) Dominant negative mutations in human PPARgamma associated with severe insulin resistance, diabetes mellitus and hypertension, Nature 402, 880-883.

[49] Silverstone, A. E., Rosenbaum, P. F., Weinstock, R. S., Bartell, S. M., Foushee, H. R., Shelton, C., and Pavuk, M. (2012) Polychlorinated biphenyl (PCB) exposure and diabetes: results from the Anniston Community Health Survey, Environ Health Perspect 120, 727-732.

[50] Wang, S. L., Tsai, P. C., Yang, C. Y., and Guo, Y. L. (2008) Increased risk of diabetes and polychlorinated biphenyls and dioxins: a 24-year follow-up study of the Yucheng cohort, Diabetes Care 31, 1574-1579.

[51] Mitoma, C., Uchi, H., Tsukimori, K., Yamada, H., Akahane, M., Imamura, T., Utani, A., and Furue, M. (2015) Yusho and its latest findings-A review in studies conducted by the Yusho Group, Environ Int 82, 41-48.

[52] Tschuor, C., Kachaylo, E., Limani, P., Raptis, D. A., Linecker, M., Tian, Y., Herrmann, U., Grabliauskaite, K., Weber, A., Columbano, A., Graf, R., Humar, B., and Clavien, P. A. (2016) Constitutive androstane receptor (Car)-driven regeneration protects liver from failure following tissue loss, J Hepatol 65, 66-74.

[53] Gruttadauria, S., Pagano, D., Liotta, R., Tropea, A., Tuzzolino, F., Marrone, G., Mamone, G., Marsh, J. W., Miraglia, R., Luca, A., Vizzini, G., and Gridelli, B. G. (2015) Liver Volume Restoration and Hepatic Microarchitecture in Small-for-Size Syndrome, Ann Transplant 20, 381-389.

[54] Michalopoulos, G. K. (2013) Principles of liver regeneration and growth homeostasis, Compr Physiol 3, 485-513.

[55] Italian Association for the Study of the, L. (2017) AISF position paper on nonalcoholic fatty liver disease (NAFLD): Updates and future directions, Dig Liver Dis 49, 471-483.

[56] Angrish, M. M., Kaiser, J. P., McQueen, C. A., and Chorley, B. N. (2016) Tipping the Balance: Hepatotoxicity and the 4 Apical Key Events of Hepatic Steatosis, Toxicol Sci 150, 261268.

[57] Stancu, C., and Sima, A. (2001) Statins: mechanism of action and effects, J Cell Mol Med 5, 378-387.

[58] Gervois, P., Torra, I. P., Fruchart, J. C., and Staels, B. (2000) Regulation of lipid and lipoprotein metabolism by PPAR activators, Clin Chem Lab Med 38, 3-11.

[59] Pernicova, I., and Korbonits, M. (2014) Metformin--mode of action and clinical implications for diabetes and cancer, Nat Rev Endocrinol 10, 143-156.

[60] Kumar, V., Abbas, A. K., Aster, J. C., Cotran, R. S., and Robbins, S. L. (2015) Robbins and Cotran Pathologic Basis of Disease, Ninth edition. ed., Elsevier/Saunders, Philadelphia, PA :.

[61] Grundy, S. M., Brewer, H. B., Jr., Cleeman, J. I., Smith, S. C., Jr., Lenfant, C., American Heart, A., National Heart, L., and Blood, I. (2004) Definition of metabolic syndrome: Report of the National Heart, Lung, and Blood Institute/American Heart Association conference on scientific issues related to definition, Circulation 109, 433-438.

[62] Internation al Diabetes Federation (2006) The IDF consensus worldwide definition of the metabolic syndrome, IDF Communications Brussels, Belgium.

[63] Prati, D., Taioli, E., Zanella, A., Della Torre, E., Butelli, S., Del Vecchio, E., Vianello, L., Zanuso, F., Mozzi, F., Milani, S., Conte, D., Colombo, M., and Sirchia, G. (2002) Updated 
definitions of healthy ranges for serum alanine aminotransferase levels, Ann Intern Med 137, 1-10.

[64] Musso, G., Gambino, R., Bo, S., Uberti, B., Biroli, G., Pagano, G., and Cassader, M. (2008) Should nonalcoholic fatty liver disease be included in the definition of metabolic syndrome? A cross-sectional comparison with Adult Treatment Panel III criteria in nonobese nondiabetic subjects, Diabetes Care 31, 562-568.

[65] Anstee, Q. M., Targher, G., and Day, C. P. (2013) Progression of NAFLD to diabetes mellitus, cardiovascular disease or cirrhosis, Nat Rev Gastroenterol Hepatol 10, 330-344.

[66] Cave, M., Falkner, K. C., Ray, M., Joshi-Barve, S., Brock, G., Khan, R., Bon Homme, M., and McClain, C. J. (2010) Toxicant-associated steatohepatitis in vinyl chloride workers, Hepatology 51, 474-481.

[67] Cuccherini, B., Nussbaum, S. J., Seeff, L. B., Lukacs, L., and Zimmerman, H. J. (1983) Stability of aspartate aminotransferase and alanine aminotransferase activities, J Lab Clin Med 102, 370-376.

[68] Williams, K. M., Williams, A. E., Kline, L. M., and Dodd, R. Y. (1987) Stability of serum alanine aminotransferase activity, Transfusion 27, 431-433.

[69] Cave, M., Falkner, K. C., Henry, L., Costello, B., Gregory, B., and McClain, C. J. (2011) Serum cytokeratin 18 and cytokine elevations suggest a high prevalence of occupational liver disease in highly exposed elastomer/polymer workers, J Occup Environ Med 53, 11281133.

[70] Aida, Y., Abe, H., Tomita, Y., Nagano, T., Seki, N., Sugita, T., Itagaki, M., Ishiguro, H., Sutoh, S., and Aizawa, Y. (2014) Serum cytokeratin 18 fragment level as a noninvasive biomarker for non-alcoholic fatty liver disease, Int J Clin Exp Med 7, 4191-4198.

[71] Feldstein, A. E., Alkhouri, N., De Vito, R., Alisi, A., Lopez, R., and Nobili, V. (2013) Serum cytokeratin-18 fragment levels are useful biomarkers for nonalcoholic steatohepatitis in children, Am J Gastroenterol 108, 1526-1531.

[72] Sumer, S., Aktug Demir, N., Kolgelier, S., Cagkan Inkaya, A., Arpaci, A., Saltuk Demir, L., and Ural, O. (2013) The Clinical Significance of Serum Apoptotic Cytokeratin 18 Neoepitope M30 (CK-18 M30) and Matrix Metalloproteinase 2 (MMP-2) Levels in Chronic Hepatitis B Patients with Cirrhosis, Hepat Mon 13, e10106.

[73] Gonzalez-Quintela, A., Abdulkader, I., Campos, J., Fernandez-Hernandez, L., and Lojo, S. (2009) Serum levels of keratin-18 fragments [tissue polypeptide-specific antigen (TPS)] are correlated with hepatocyte apoptosis in alcoholic hepatitis, Dig Dis Sci 54, 648-653.

[74] Hermanson, M. H., Scholten, C. A., and Compher, K. (2003) Variable air temperature response of gas-phase atmospheric polychlorinated biphenyls near a former manufacturing facility, Environmental science \& technology 37, 4038-4042.

[75] Hermanson, M. H., and Johnson, G. W. (2007) Polychlorinated biphenyls in tree bark near a former manufacturing plant in Anniston, Alabama, Chemosphere 68, 191-198.

[76] Goncharov, A., Bloom, M., Pavuk, M., Birman, I., and Carpenter, D. O. (2010) Blood pressure and hypertension in relation to levels of serum polychlorinated biphenyls in residents of Anniston, Alabama, J Hypertens 28, 2053-2060.

[77] Registry, A. f. T. S. a. D. (2014) Anniston Community Health Survey, Agency for Toxic Substances and Disease Registry, 4770 Buford Hwy NE Atlanta, GA 30341 USA.

[78] Pavuk, M., Olson, J. R., Sjodin, A., Wolff, P., Turner, W. E., Shelton, C., Dutton, N. D., Bartell, S., and Anniston Environmental Health Research, C. (2014) Serum concentrations of polychlorinated biphenyls (PCBs) in participants of the Anniston Community Health Survey, Sci Total Environ 473-474, 286-297. 
[79] Aminov, Z., Haase, R. F., Pavuk, M., Carpenter, D. O., and Anniston Environmental Health Research, C. (2013) Analysis of the effects of exposure to polychlorinated biphenyls and chlorinated pesticides on serum lipid levels in residents of Anniston, Alabama, Environ Health 12, 108.

[80] Birnbaum, L. S., Dutton, N. D., Cusack, C., Mennemeyer, S. T., and Pavuk, M. (2016) Anniston community health survey: Follow-up and dioxin analyses (ACHS-II)--methods, Environ Sci Pollut Res Int 23, 2014-2021.

[81] Pavuk, M., Olson, J. R., Wattigney, W. A., Dutton, N. D., Sjodin, A., Shelton, C., Turner, W. E., Bartell, S. M., and Anniston Environmental Health Research, C. (2014) Predictors of serum polychlorinated biphenyl concentrations in Anniston residents, Sci Total Environ 496, 624-634.

[82] Goncharov, A., Pavuk, M., Foushee, H. R., Carpenter, D. O., and Anniston Environmental Health Reseach, C. (2011) Blood pressure in relation to concentrations of PCB congeners and chlorinated pesticides, Environ Health Perspect 119, 319-325.

[83] Joshi-Barve, S., Kirpich, I., Cave, M. C., Marsano, L. S., and McClain, C. J. (2015) Alcoholic, Nonalcoholic, and Toxicant-Associated Steatohepatitis: Mechanistic Similarities and Differences, Cell Mol Gastroenterol Hepatol 1, 356-367.

[84] Wahlang, B., Beier, J. I., Clair, H. B., Bellis-Jones, H. J., Falkner, K. C., McClain, C. J., and Cave, M. C. (2013) Toxicant-associated steatohepatitis, Toxicol Pathol 41, 343-360.

[85] Wallace, T. M., Levy, J. C., and Matthews, D. R. (2004) Use and abuse of HOMA modeling, Diabetes Care 27, 1487-1495.

[86] Hornung, R. W., and Reed, L. D. (1990) Estimation of Average Concentration in the Presence of Nondetectable Values, Applied Occupational and Environmental Hygiene 5, 46-51.

[87] Bernert, J. T., Turner, W. E., Patterson, D. G., Jr., and Needham, L. L. (2007) Calculation of serum "total lipid" concentrations for the adjustment of persistent organohalogen toxicant measurements in human samples, Chemosphere 68, 824-831.

[88] Matthews, D. R., Hosker, J. P., Rudenski, A. S., Naylor, B. A., Treacher, D. F., and Turner, R. C. (1985) Homeostasis model assessment: insulin resistance and beta-cell function from fasting plasma glucose and insulin concentrations in man, Diabetologia 28, 412-419.

[89] Feldstein, A. E., Wieckowska, A., Lopez, A. R., Liu, Y. C., Zein, N. N., and McCullough, A. J. (2009) Cytokeratin-18 fragment levels as noninvasive biomarkers for nonalcoholic steatohepatitis: a multicenter validation study, Hepatology 50, 1072-1078.

[90] Pan, J. J., and Fallon, M. B. (2014) Gender and racial differences in nonalcoholic fatty liver disease, World J Hepatol 6, 274-283.

[91] Syn, W. K., Choi, S. S., and Diehl, A. M. (2009) Apoptosis and cytokines in non-alcoholic steatohepatitis, Clin Liver Dis 13, 565-580.

[92] Syn, W. K., Teaberry, V., Choi, S. S., and Diehl, A. M. (2009) Similarities and differences in the pathogenesis of alcoholic and nonalcoholic steatohepatitis, Semin Liver Dis 29, 200210.

[93] Younossi, Z. M., Koenig, A. B., Abdelatif, D., Fazel, Y., Henry, L., and Wymer, M. (2016) Global epidemiology of nonalcoholic fatty liver disease-Meta-analytic assessment of prevalence, incidence, and outcomes, Hepatology 64, 73-84.

[94] Stern, S. E., Williams, K., Ferrannini, E., DeFronzo, R. A., Bogardus, C., and Stern, M. P. (2005) Identification of individuals with insulin resistance using routine clinical measurements, Diabetes 54, 333-339.

[95] Serdar, B., LeBlanc, W. G., Norris, J. M., and Dickinson, L. M. (2014) Potential effects of polychlorinated biphenyls (PCBs) and selected organochlorine pesticides (OCPs) on 
immune cells and blood biochemistry measures: a cross-sectional assessment of the NHANES 2003-2004 data, Environ Health 13, 114.

[96] Kumar, J., Lind, L., Salihovic, S., van Bavel, B., Ingelsson, E., and Lind, P. M. (2014) Persistent organic pollutants and liver dysfunction biomarkers in a population-based human sample of men and women, Environ Res 134, 251-256.

[97] Yorita Christensen, K. L., Carrico, C. K., Sanyal, A. J., and Gennings, C. (2013) Multiple classes of environmental chemicals are associated with liver disease: NHANES 2003-2004, Int J Hyg Environ Health 216, 703-709.

[98] Kim, M. J., Marchand, P., Henegar, C., Antignac, J. P., Alili, R., Poitou, C., Bouillot, J. L., Basdevant, A., Le Bizec, B., Barouki, R., and Clement, K. (2011) Fate and complex pathogenic effects of dioxins and polychlorinated biphenyls in obese subjects before and after drastic weight loss, Environ Health Perspect 119, 377-383.

[99] Rantakokko, P., Mannisto, V., Airaksinen, R., Koponen, J., Viluksela, M., Kiviranta, H., and Pihlajamaki, J. (2015) Persistent organic pollutants and non-alcoholic fatty liver disease in morbidly obese patients: a cohort study, Environ Health 14, 79.

[100] Perkins, J. T., Petriello, M. C., Newsome, B. J., and Hennig, B. (2016) Polychlorinated biphenyls and links to cardiovascular disease, Environ Sci Pollut Res Int 23, 2160-2172.

[101] A.T.S.D.R. (2000) Toxicological Profile For Polychlorinated Biphenyls (PCBs), (Services, U. S. D. o. H. a. H., Ed.), ATSDR Division of Toxicology/Toxicology Information Branch, Atlanta, GA.

[102] National Toxicology, P., and National Institutes of, H. (2006) NTP technical report on the toxicology and carcinogenesis studies of 3,3', 4, 4',5-Pentachlorobiphenyl (PCB 126) (CAS NO. 57465-28-8) in female Harlan Sprague-Dawley rats : (gavage studies), National Institutes of Health, Public Health Service, U.S. Department of Health and Human Services, [Bethesda, Md.] :.

[103] Walker, N. J., National Toxicology, P., and National Institutes of, H. (2010) NTP technical report on the toxicology and carcinogenesis studies of 2,3',4,4',5-pentachlorobiphenyl (PCB 118) [CAS no. 31508-00-6] in female Harlan Sprague-Dawley rats : (gavage studies), National Institutes of Health, Public Health Service, U.S. Dept. of Health and Human Services, Research Triangle Park, NC :.

[104] N.C.H.S. (2003-2004) NHANES 2003-2004 Laboratory Procedure Manual: Alanine Amino Transferase (ALT) in Refrigerated Serum., Available: https://www.cdc.gov/nchs/data/nhanes/nhanes 03 04/140 c met alanine amino tra nsferase.pdf.

[105] Warner, J., Osuch, J. R., Karmaus, W., Landgraf, J. R., Taffe, B., O'Keefe, M., Mikucki, D., and Haan, P. (2012) Common classification schemes for PCB congeners and the gene expression of CYP17, CYP19, ESR1 and ESR2, Sci Total Environ 414, 81-89.

[106] Beilke, L. D., Aleksunes, L. M., Olson, E. R., Besselsen, D. G., Klaassen, C. D., Dvorak, K., and Cherrington, N. J. (2009) Decreased apoptosis during CAR-mediated hepatoprotection against lithocholic acid-induced liver injury in mice, Toxicol Lett 188, 38-44.

[107] Shi, X., Wahlang, B., Wei, X., Yin, X., Falkner, K. C., Prough, R. A., Kim, S. H., Mueller, E. G., McClain, C. J., Cave, M., and Zhang, X. (2012) Metabolomic analysis of the effects of polychlorinated biphenyls in nonalcoholic fatty liver disease, Journal of proteome research 11, 3805-3815.

[108] Wahlang, B., Prough, R. A., Falkner, K. C., Hardesty, J. E., Song, M., Clair, H. B., Clark, B. J., States, J. C., Arteel, G. E., and Cave, M. C. (2016) Polychlorinated Biphenyl-Xenobiotic Nuclear Receptor Interactions Regulate Energy Metabolism, Behavior, and Inflammation in Non-alcoholic-Steatohepatitis, Toxicol Sci 149, 396-410. 
[109] Hennig, B., Reiterer, G., Toborek, M., Matveev, S. V., Daugherty, A., Smart, E., and Robertson, L. W. (2005) Dietary fat interacts with PCBs to induce changes in lipid metabolism in mice deficient in low-density lipoprotein receptor, Environ Health Perspect 113, 83-87.

[110] Li, M. C., Chen, P. C., Tsai, P. C., Furue, M., Onozuka, D., Hagihara, A., Uchi, H., Yoshimura, T., and Guo, Y. L. (2015) Mortality after exposure to polychlorinated biphenyls and polychlorinated dibenzofurans: a meta-analysis of two highly exposed cohorts, Int J Cancer 137, 1427-1432.

[111] Anstee, Q. M., and Day, C. P. (2013) The genetics of NAFLD, Nat Rev Gastroenterol Hepatol 10, 645-655.

[112] Houghton-Rahrig, L., Schutte, D., Fenton, J. I., and Awad, J. (2014) Nonalcoholic fatty liver disease and the PNPLA3 gene, Medsurg nursing : official journal of the Academy of Medical-Surgical Nurses 23, 101-106, 121.

[113] Sookoian, S., Castano, G. O., Burgueno, A. L., Gianotti, T. F., Rosselli, M. S., and Pirola, C. J. (2010) The nuclear receptor PXR gene variants are associated with liver injury in nonalcoholic fatty liver disease, Pharmacogenet Genomics 20, 1-8.

[114] Kumar, J., Lind, P. M., Salihovic, S., van Bavel, B., Ingelsson, E., and Lind, L. (2014) Persistent organic pollutants and inflammatory markers in a cross-sectional study of elderly Swedish people: the PIVUS cohort, Environ Health Perspect 122, 977-983.

[115] Lim, J. E., and Jee, S. H. (2015) Association between serum levels of adiponectin and polychlorinated biphenyls in Korean men and women, Endocrine 48, 211-217.

[116] Turyk, M., Fantuzzi, G., Persky, V., Freels, S., Lambertino, A., Pini, M., Rhodes, D. H., and Anderson, H. A. (2015) Persistent organic pollutants and biomarkers of diabetes risk in a cohort of Great Lakes sport caught fish consumers, Environ Res 140, 335-344.

[117] Ghosh, S., Trnovec, T., Palkovicova, L., Hoffman, E. P., Washington, K., and Dutta, S. K. (2013) Status of LEPR Gene in PCB-exposed Population: A Quick Look, Int J Hum Genet 13, 27-32.

[118] Ferrante, M. C., Amero, P., Santoro, A., Monnolo, A., Simeoli, R., Di Guida, F., Mattace Raso, G., and Meli, R. (2014) Polychlorinated biphenyls (PCB 101, PCB 153 and PCB 180) alter leptin signaling and lipid metabolism in differentiated 3T3-L1 adipocytes, Toxicol Appl Pharmacol 279, 401-408.

[119] Ewald, N., and Bretzel, R. G. (2013) Diabetes mellitus secondary to pancreatic diseases (Type 3c)--are we neglecting an important disease?, Eur J Intern Med 24, 203-206.

[120] Castera, L. (2015) Noninvasive Evaluation of Nonalcoholic Fatty Liver Disease, Semin Liver Dis 35, 291-303.

[121] Al-Eryani, L., Wahlang, B., Falkner, K. C., Guardiola, J. J., Clair, H. B., Prough, R. A., and Cave, M. (2015) Identification of Environmental Chemicals Associated with the Development of Toxicant-associated Fatty Liver Disease in Rodents, Toxicol Pathol 43, 482-497.

[122] Trapnell, C., Roberts, A., Goff, L., Pertea, G., Kim, D., Kelley, D. R., Pimentel, H., Salzberg, S. L., Rinn, J. L., and Pachter, L. (2012) Differential gene and transcript expression analysis of RNA-seq experiments with TopHat and Cufflinks, Nature protocols 7, 562-578.

[123] Zhang, H., Wu, J., and Yu, L. (2014) Association of Gln27Glu and Arg16Gly polymorphisms in Beta2-adrenergic receptor gene with obesity susceptibility: a meta-analysis, PLoS One 9, e100489.

[124] Moreno-Navarrete, J. M., Botas, P., Valdes, S., Ortega, F. J., Delgado, E., Vazquez-Martin, A., Bassols, J., Pardo, G., Ricart, W., Menendez, J. A., and Fernandez-Real, J. M. (2009) 
Val1483lle in FASN gene is linked to central obesity and insulin sensitivity in adult white men, Obesity (Silver Spring) 17, 1755-1761.

[125] Karim, S., Adams, D. H., and Lalor, P. F. (2012) Hepatic expression and cellular distribution of the glucose transporter family, World J Gastroenterol 18, 6771-6781.

[126] Du, K., Herzig, S., Kulkarni, R. N., and Montminy, M. (2003) TRB3: a tribbles homolog that inhibits Akt/PKB activation by insulin in liver, Science 300, 1574-1577.

[127] Marinho, R., Ropelle, E. R., Cintra, D. E., De Souza, C. T., Da Silva, A. S., Bertoli, F. C., Colantonio, E., D'Almeida, V., and Pauli, J. R. (2012) Endurance exercise training increases APPL1 expression and improves insulin signaling in the hepatic tissue of dietinduced obese mice, independently of weight loss, J Cell Physiol 227, 2917-2926.

[128] Liu, W. J., Ma, L. Q., Liu, W. H., Zhou, W., Zhang, K. Q., and Zou, C. G. (2011) Inhibition of hepatic glycogen synthesis by hyperhomocysteinemia mediated by TRB3, Am J Pathol $178,1489-1499$.

[129] Li, K., Xiao, Y., Yu, J., Xia, T., Liu, B., Guo, Y., Deng, J., Chen, S., Wang, C., and Guo, F. (2016) Liver-specific Gene Inactivation of the Transcription Factor ATF4 Alleviates Alcoholic Liver Steatosis in Mice, J Biol Chem 291, 18536-18546.

[130] Prudente, S., and Trischitta, V. (2015) The TRIB3 Q84R polymorphism, insulin resistance and related metabolic alterations, Biochem Soc Trans 43, 1108-1111.

[131] Zheng, Z., Kim, H., Qiu, Y., Chen, X., Mendez, R., Dandekar, A., Zhang, X., Zhang, C., Liu, A. C., Yin, L., Lin, J. D., Walker, P. D., Kapatos, G., and Zhang, K. (2016) CREBH Couples Circadian Clock With Hepatic Lipid Metabolism, Diabetes 65, 3369-3383.

[132] Ohno, T., Onishi, Y., and Ishida, N. (2007) A novel E4BP4 element drives circadian expression of mPeriod2, Nucleic Acids Res 35, 648-655.

[133] Wolk, R., and Somers, V. K. (2007) Sleep and the metabolic syndrome, Exp Physiol 92, 6778.

[134] Martins, R. C., Andersen, M. L., and Tufik, S. (2008) The reciprocal interaction between sleep and type 2 diabetes mellitus: facts and perspectives, Braz J Med Biol Res 41, 180187.

[135] Damiola, F., Le Minh, N., Preitner, N., Kornmann, B., Fleury-Olela, F., and Schibler, U. (2000) Restricted feeding uncouples circadian oscillators in peripheral tissues from the central pacemaker in the suprachiasmatic nucleus, Genes Dev 14, 2950-2961.

[136] Eckel-Mahan, K. L., Patel, V. R., de Mateo, S., Orozco-Solis, R., Ceglia, N. J., Sahar, S., DilagPenilla, S. A., Dyar, K. A., Baldi, P., and Sassone-Corsi, P. (2013) Reprogramming of the circadian clock by nutritional challenge, Cell 155, 1464-1478.

[137] Depner, C. M., Stothard, E. R., and Wright, K. P., Jr. (2014) Metabolic consequences of sleep and circadian disorders, Curr Diab Rep 14, 507.

[138] Wang, X. S., Armstrong, M. E., Cairns, B. J., Key, T. J., and Travis, R. C. (2011) Shift work and chronic disease: the epidemiological evidence, Occup Med (Lond) 61, 78-89.

[139] Sporl, F., Korge, S., Jurchott, K., Wunderskirchner, M., Schellenberg, K., Heins, S., Specht, A., Stoll, C., Klemz, R., Maier, B., Wenck, H., Schrader, A., Kunz, D., Blatt, T., and Kramer, A. (2012) Kruppel-like factor 9 is a circadian transcription factor in human epidermis that controls proliferation of keratinocytes, Proc Natl Acad Sci U S A 109, 10903-10908.

[140] Zeisberg, M., and Neilson, E. G. (2009) Biomarkers for epithelial-mesenchymal transitions, J Clin Invest 119, 1429-1437.

[141] Jou, J., and Diehl, A. M. (2010) Epithelial-mesenchymal transitions and hepatocarcinogenesis, J Clin Invest 120, 1031-1034.

[142] Fleig, S. V., Choi, S. S., Yang, L., Jung, Y., Omenetti, A., VanDongen, H. M., Huang, J., Sicklick, J. K., and Diehl, A. M. (2007) Hepatic accumulation of Hedgehog-reactive 
progenitors increases with severity of fatty liver damage in mice, Lab Invest 87, 12271239.

[143] Verdelho Machado, M., and Diehl, A. M. (2016) Role of Hedgehog Signaling Pathway in NASH, Int J Mol Sci 17.

[144] Giannelli, G., Mikulits, W., Dooley, S., Fabregat, I., Moustakas, A., ten Dijke, P., Portincasa, P., Winter, P., Janssen, R., Leporatti, S., Herrera, B., and Sanchez, A. (2016) The rationale for targeting TGF-beta in chronic liver diseases, Eur J Clin Invest 46, 349-361.

[145] Miao, C. G., Yang, Y. Y., He, X., Huang, C., Huang, Y., Zhang, L., Lv, X. W., Jin, Y., and Li, J. (2013) Wnt signaling in liver fibrosis: progress, challenges and potential directions, Biochimie 95, 2326-2335.

[146] Wang, S., Song, K., Srivastava, R., Dong, C., Go, G. W., Li, N., Iwakiri, Y., and Mani, A. (2015) Nonalcoholic fatty liver disease induced by noncanonical Wnt and its rescue by Wnt3a, FASEB J 29, 3436-3445.

[147] Machado, M. V., Michelotti, G. A., Pereira, T. A., Xie, G., Premont, R., Cortez-Pinto, H., and Diehl, A. M. (2015) Accumulation of duct cells with activated YAP parallels fibrosis progression in non-alcoholic fatty liver disease, $J$ Hepatol 63, 962-970.

[148] Pavuk, M., Olson, J. R., Sjodin, A., Wolff, P., Turner, W. E., Shelton, C., Dutton, N. D., Bartell, S., and Anniston Environmental Health Research, C. (2014) Serum concentrations of polychlorinated biphenyls (PCBs) in participants of the Anniston Community Health Survey, Sci. Total Environ. 473-474, 286-297.

[149] Wormke, M., Stoner, M., Saville, B., Walker, K., Abdelrahim, M., Burghardt, R., and Safe, S. (2003) The aryl hydrocarbon receptor mediates degradation of estrogen receptor alpha through activation of proteasomes, Mol Cell Biol 23, 1843-1855.

[150] Matthews, J., and Gustafsson, J. A. (2006) Estrogen receptor and aryl hydrocarbon receptor signaling pathways, Nucl Recept Signal 4, e016.

[151] Mitro, N., Vargas, L., Romeo, R., Koder, A., and Saez, E. (2007) T0901317 is a potent PXR ligand: implications for the biology ascribed to LXR, FEBS Lett 581, 1721-1726.

[152] Kodama, S., Koike, C., Negishi, M., and Yamamoto, Y. (2004) Nuclear receptors CAR and PXR cross talk with FOXO1 to regulate genes that encode drug-metabolizing and gluconeogenic enzymes, Mol Cell Biol 24, 7931-7940.

[153] Quack, M., Frank, C., and Carlberg, C. (2002) Differential nuclear receptor signalling from DR4-type response elements, J Cell Biochem 86, 601-612.

[154] Grefhorst, A., Elzinga, B. M., Voshol, P. J., Plosch, T., Kok, T., Bloks, V. W., van der Sluijs, F. H., Havekes, L. M., Romijn, J. A., Verkade, H. J., and Kuipers, F. (2002) Stimulation of lipogenesis by pharmacological activation of the liver $X$ receptor leads to production of large, triglyceride-rich very low density lipoprotein particles, J Biol Chem 277, 3418234190.

[155] Safe, S. (1997) Limitations of the toxic equivalency factor approach for risk assessment of TCDD and related compounds, Teratog Carcinog Mutagen 17, 285-304.

[156] Murray, I. A., Morales, J. L., Flaveny, C. A., Dinatale, B. C., Chiaro, C., Gowdahalli, K., Amin, S., and Perdew, G. H. (2010) Evidence for ligand-mediated selective modulation of aryl hydrocarbon receptor activity, Mol Pharmacol 77, 247-254.

[157] Coombes, J. D., Swiderska-Syn, M., Dolle, L., Reid, D., Eksteen, B., Claridge, L., BrionesOrta, M. A., Shetty, S., Oo, Y. H., Riva, A., Chokshi, S., Papa, S., Mi, Z., Kuo, P. C., Williams, R., Canbay, A., Adams, D. H., Diehl, A. M., van Grunsven, L. A., Choi, S. S., and Syn, W. K. (2015) Osteopontin neutralisation abrogates the liver progenitor cell response and fibrogenesis in mice, Gut 64, 1120-1131. 
[158] Sui, Y., Zheng, X., and Zhao, D. (2015) Rab31 promoted hepatocellular carcinoma (HCC) progression via inhibition of cell apoptosis induced by PI3K/AKT/Bcl-2/BAX pathway, Tumour Biol 36, 8661-8670.

[159] Yagai, T., Miyajima, A., and Tanaka, M. (2014) Semaphorin 3E secreted by damaged hepatocytes regulates the sinusoidal regeneration and liver fibrosis during liver regeneration, Am J Pathol 184, 2250-2259.

[160] Shan, Q., Huang, F., Wang, J., and Du, Y. (2015) Effects of co-exposure to 2,3,7,8tetrachlorodibenzo-p-dioxin and polychlorinated biphenyls on nonalcoholic fatty liver disease in mice, Environ Toxicol 30, 1364-1374.

[161] Li, Y., Zalzala, M., Jadhav, K., Xu, Y., Kasumov, T., Yin, L., and Zhang, Y. (2016) Carboxylesterase 2 prevents liver steatosis by modulating lipolysis, endoplasmic reticulum stress, and lipogenesis and is regulated by hepatocyte nuclear factor 4 alpha in mice, Hepatology 63, 1860-1874.

[162] Volat, F. E., Pointud, J. C., Pastel, E., Morio, B., Sion, B., Hamard, G., Guichardant, M., Colas, R., Lefrancois-Martinez, A. M., and Martinez, A. (2012) Depressed levels of prostaglandin F2alpha in mice lacking Akr1b7 increase basal adiposity and predispose to diet-induced obesity, Diabetes 61, 2796-2806.

[163] Aigner, E., Theurl, I., Theurl, M., Lederer, D., Haufe, H., Dietze, O., Strasser, M., Datz, C., and Weiss, G. (2008) Pathways underlying iron accumulation in human nonalcoholic fatty liver disease, Am J Clin Nutr 87, 1374-1383.

[164] Smalling, R. L., Delker, D. A., Zhang, Y., Nieto, N., McGuiness, M. S., Liu, S., Friedman, S. L., Hagedorn, C. H., and Wang, L. (2013) Genome-wide transcriptome analysis identifies novel gene signatures implicated in human chronic liver disease, Am J Physiol Gastrointest Liver Physiol 305, G364-374.

[165] Gao, Y., Liu, Y., Yang, M., Guo, X., Zhang, M., Li, H., Li, J., and Zhao, J. (2016) IL-33 treatment attenuated diet-induced hepatic steatosis but aggravated hepatic fibrosis, Oncotarget 7, 33649-33661.

[166] King, J. A., Corcoran, N. M., D'Abaco, G. M., Straffon, A. F., Smith, C. T., Poon, C. L., Buchert, M., I, S., Hall, N. E., Lock, P., and Hovens, C. M. (2006) Eve-3: a liver enriched suppressor of Ras/MAPK signaling, J Hepatol 44, 758-767.

[167] Yoshida, T., Hisamoto, T., Akiba, J., Koga, H., Nakamura, K., Tokunaga, Y., Hanada, S., Kumemura, H., Maeyama, M., Harada, M., Ogata, H., Yano, H., Kojiro, M., Ueno, T., Yoshimura, A., and Sata, M. (2006) Spreds, inhibitors of the Ras/ERK signal transduction, are dysregulated in human hepatocellular carcinoma and linked to the malignant phenotype of tumors, Oncogene 25, 6056-6066.

[168] Ding, L., Wang, Z., Yan, J., Yang, X., Liu, A., Qiu, W., Zhu, J., Han, J., Zhang, H., Lin, J., Cheng, L., Qin, X., Niu, C., Yuan, B., Wang, X., Zhu, C., Zhou, Y., Li, J., Song, H., Huang, C., and Ye, Q. (2009) Human four-and-a-half LIM family members suppress tumor cell growth through a TGF-beta-like signaling pathway, J Clin Invest 119, 349-361.

[169] Chua, C. E., and Tang, B. L. (2014) Engagement of the small GTPase Rab31 protein and its effector, early endosome antigen 1 , is important for trafficking of the ligand-bound epidermal growth factor receptor from the early to the late endosome, J Biol Chem 289, 12375-12389.

[170] Chua, C. E., and Tang, B. L. (2015) The role of the small GTPase Rab31 in cancer, J Cell Mol Med 19, 1-10.

[171] Huang, J., Ye, X., Guan, J., Chen, B., Li, Q., Zheng, X., Liu, L., Wang, S., Ding, Y., Ding, Y., and Chen, L. (2013) Tiam1 is associated with hepatocellular carcinoma metastasis, Int J Cancer 132, 90-100. 
[172] Guo, X., Wang, M., Zhao, Y., Wang, X., Shen, M., Zhu, F., Shi, C., Xu, M., Li, X., Peng, F., Zhang, H., Feng, Y., Xie, Y., Xu, X., Jia, W., He, R., Jiang, J., Hu, J., Tian, R., and Qin, R. (2016) Par3 regulates invasion of pancreatic cancer cells via interaction with Tiam1, Clin Exp Med 16, 357-365.

[173] Liu, Y., Ding, Y., Huang, J., Wang, S., Ni, W., Guan, J., Li, Q., Zhang, Y., Ding, Y., Chen, B., and Chen, L. (2014) MiR-141 suppresses the migration and invasion of HCC cells by targeting Tiam1, PLoS One 9, e88393.

[174] Chen, G., Lu, L., Liu, C., Shan, L., and Yuan, D. (2015) MicroRNA-377 suppresses cell proliferation and invasion by inhibiting TIAM1 expression in hepatocellular carcinoma, PLoS One 10, e0117714.

[175] Ahn, S. G., Kim, H. S., Jeong, S. W., Kim, B. E., Rhim, H., Shim, J. Y., Kim, J. W., Lee, J. H., and Kim, I. K. (2002) Sox-4 is a positive regulator of Hep3B and HepG2 cells' apoptosis induced by prostaglandin (PG)A(2) and delta(12)-PGJ(2), Exp Mol Med 34, 243-249.

[176] Fan, X. P., Ji, X. F., Li, X. Y., Gao, S., Fan, Y. C., and Wang, K. (2016) Methylation of the Glutathione-S-Transferase P1 Gene Promoter Is Associated with Oxidative Stress in Patients with Chronic Hepatitis B, Tohoku J Exp Med 238, 57-64.

[177] Nakagawa, Y., Aoki, N., Aoyama, K., Shimizu, H., Shimano, H., Yamada, N., and Miyazaki, H. (2005) Receptor-type protein tyrosine phosphatase epsilon (PTPepsilonM) is a negative regulator of insulin signaling in primary hepatocytes and liver, Zoolog Sci 22, 169-175.

[178] Dong, Y., Li, K., Xu, Z., Ma, H., Zheng, J., Hu, Z., He, S., Wu, Y., Sun, Z., Luo, L., Li, J., Zhang, $H_{.}$, and Zhang, X. (2015) Exploration of the linkage elements of porcupine antagonists led to potent Wnt signaling pathway inhibitors, Bioorg Med Chem 23, 6855-6868.

[179] Radanovic, T., Wagner, C. A., Murer, H., and Biber, J. (2005) Regulation of intestinal phosphate transport. I. Segmental expression and adaptation to low-P(i) diet of the type Ilb Na(+)-P(i) cotransporter in mouse small intestine, Am J Physiol Gastrointest Liver Physiol 288, G496-500.

[180] Wagner, C. A., Hernando, N., Forster, I. C., and Biber, J. (2014) The SLC34 family of sodiumdependent phosphate transporters, Pflugers Arch 466, 139-153.

[181] Giera, S., Braeuning, A., Kohle, C., Bursch, W., Metzger, U., Buchmann, A., and Schwarz, M. (2010) Wnt/beta-catenin signaling activates and determines hepatic zonal expression of glutathione S-transferases in mouse liver, Toxicol Sci 115, 22-33.

[182] Schmidt, D. R., Schmidt, S., Holmstrom, S. R., Makishima, M., Yu, R. T., Cummins, C. L., Mangelsdorf, D. J., and Kliewer, S. A. (2011) AKR1B7 is induced by the farnesoid X receptor and metabolizes bile acids, J Biol Chem 286, 2425-2432.

[183] Liu, M. J., Takahashi, Y., Wada, T., He, J., Gao, J., Tian, Y., Li, S., and Xie, W. (2009) The aldoketo reductase Akr1b7 gene is a common transcriptional target of xenobiotic receptors pregnane $X$ receptor and constitutive androstane receptor, Mol Pharmacol 76, 604-611.

[184] Moylan, C. A., Pang, H., Dellinger, A., Suzuki, A., Garrett, M. E., Guy, C. D., Murphy, S. K., Ashley-Koch, A. E., Choi, S. S., Michelotti, G. A., Hampton, D. D., Chen, Y., Tillmann, H. L., Hauser, M. A., Abdelmalek, M. F., and Diehl, A. M. (2014) Hepatic gene expression profiles differentiate presymptomatic patients with mild versus severe nonalcoholic fatty liver disease, Hepatology 59, 471-482.

[185] Suh, Y., Yoon, C. H., Kim, R. K., Lim, E. J., Oh, Y. S., Hwang, S. G., An, S., Yoon, G., Gye, M. C., Yi, J. M., Kim, M. J., and Lee, S. J. (2013) Claudin-1 induces epithelial-mesenchymal transition through activation of the c-Abl-ERK signaling pathway in human liver cells, Oncogene 32, 4873-4882. 
[186] Erdelyi-Belle, B., Torok, G., Apati, A., Sarkadi, B., Schaff, Z., Kiss, A., and Homolya, L. (2015) Expression of Tight Junction Components in Hepatocyte-Like Cells Differentiated from Human Embryonic Stem Cells, Pathol Oncol Res 21, 1059-1070.

[187] Choi, S. S., and Diehl, A. M. (2009) Epithelial-to-mesenchymal transitions in the liver, Hepatology 50, 2007-2013.

[188] Kim, J. T., and Lee, H. K. (2014) Metabolic syndrome and the environmental pollutants from mitochondrial perspectives, Rev Endocr Metab Disord 15, 253-262.

[189] Vandenberg, L. N., Colborn, T., Hayes, T. B., Heindel, J. J., Jacobs, D. R., Jr., Lee, D. H., Shioda, T., Soto, A. M., vom Saal, F. S., Welshons, W. V., Zoeller, R. T., and Myers, J. P. (2012) Hormones and endocrine-disrupting chemicals: low-dose effects and nonmonotonic dose responses, Endocr Rev 33, 378-455.

[190] Harper, N., Connor, K., Steinberg, M., and Safe, S. (1995) Immunosuppressive activity of polychlorinated biphenyl mixtures and congeners: nonadditive (antagonistic) interactions, Fundam Appl Toxicol 27, 131-139.

[191] Cieslak, M., Wojtczak, A., and Cieslak, M. (2015) Role of pro-inflammatory cytokines of pancreatic islets and prospects of elaboration of new methods for the diabetes treatment, Acta Biochim Pol 62, 15-21.

[192] Howard, J. K., and Flier, J. S. (2006) Attenuation of leptin and insulin signaling by SOCS proteins, Trends Endocrinol Metab 17, 365-371.

[193] Wang, Y., van Boxel-Dezaire, A. H., Cheon, H., Yang, J., and Stark, G. R. (2013) STAT3 activation in response to IL- 6 is prolonged by the binding of IL- 6 receptor to EGF receptor, Proc Natl Acad Sci U S A 110, 16975-16980.

[194] Wunderlich, F. T., Strohle, P., Konner, A. C., Gruber, S., Tovar, S., Bronneke, H. S., JunttiBerggren, L., Li, L. S., van Rooijen, N., Libert, C., Berggren, P. O., and Bruning, J. C. (2010) Interleukin-6 signaling in liver-parenchymal cells suppresses hepatic inflammation and improves systemic insulin action, Cell Metab 12, 237-249.

[195] Vincent, T., Molina, L., Espert, L., and Mechti, N. (2003) Hyaluronan, a major non-protein glycosaminoglycan component of the extracellular matrix in human bone marrow, mediates dexamethasone resistance in multiple myeloma, Br J Haematol 121, 259-269.

[196] Xu, C. X., Krager, S. L., Liao, D. F., and Tischkau, S. A. (2010) Disruption of CLOCK-BMAL1 transcriptional activity is responsible for aryl hydrocarbon receptor-mediated regulation of Period1 gene, Toxicol Sci 115, 98-108.

[197] Kodama, S., Moore, R., Yamamoto, Y., and Negishi, M. (2007) Human nuclear pregnane X receptor cross-talk with CREB to repress CAMP activation of the glucose-6-phosphatase gene, Biochem J 407, 373-381.

[198] Poitz, D. M., Ende, G., Stutz, B., Augstein, A., Friedrichs, J., Brunssen, C., Werner, C., Strasser, R. H., and Jellinghaus, S. (2015) EphrinB2/EphA4-mediated activation of endothelial cells increases monocyte adhesion, Mol Immunol 68, 648-656.

[199] Pasquale, E. B. (2008) Eph-ephrin bidirectional signaling in physiology and disease, Cell 133, 38-52.

[200] Zygmunt, M., Wienhard, J., Boving, B., Munstedt, K., Braems, G., Bohle, R. M., and Lang, U. (1998) [Expression of cell adhesion molecules in the extravillous trophoblast in placentas of preterm pregnancies and in placentas at term], Zentralbl Gynakol 120, 488-492.

[201] Lisabeth, E. M., Falivelli, G., and Pasquale, E. B. (2013) Eph receptor signaling and ephrins, Cold Spring Harb Perspect Biol 5.

[202] Hong, J. Y., Shin, M. H., Douglas, I. S., Chung, K. S., Kim, E. Y., Jung, J. Y., Kang, Y. A., Kim, S. K., Chang, J., Kim, Y. S., and Park, M. S. (2016) Inhibition of EphA2/EphrinA1 signal attenuates lipopolysaccharide-induced lung injury, Clin Sci (Lond) 130, 1993-2003. 
[203] Li, G., Ji, X. D., Gao, H., Zhao, J. S., Xu, J. F., Sun, Z. J., Deng, Y. Z., Shi, S., Feng, Y. X., Zhu, Y. Q., Wang, T., Li, J. J., and Xie, D. (2012) EphB3 suppresses non-small-cell lung cancer metastasis via a PP2A/RACK1/Akt signalling complex, Nat Commun 3, 667.

[204] Zantek, N. D., Azimi, M., Fedor-Chaiken, M., Wang, B., Brackenbury, R., and Kinch, M. S. (1999) E-cadherin regulates the function of the EphA2 receptor tyrosine kinase, Cell Growth Differ 10, 629-638.

[205] Konstantinova, I., Nikolova, G., Ohara-Imaizumi, M., Meda, P., Kucera, T., Zarbalis, K., Wurst, W., Nagamatsu, S., and Lammert, E. (2007) EphA-Ephrin-A-mediated beta cell communication regulates insulin secretion from pancreatic islets, Cell 129, 359-370.

[206] Sims, P., Grover, P. L., Swaisland, A., Pal, K., and Hewer, A. (1974) Metabolic activation of benzo(a)pyrene proceeds by a diol-epoxide, Nature 252, 326-328.

[207] Ding, Y., Chen, B., Wang, S., Zhao, L., Chen, J., Ding, Y., Chen, L., and Luo, R. (2009) Overexpression of Tiam1 in hepatocellular carcinomas predicts poor prognosis of HCC patients, Int J Cancer 124, 653-658.

[208] Yang, W., Lv, S., Liu, X., Liu, H., Yang, W., and Hu, F. (2010) Up-regulation of Tiam1 and Rac1 correlates with poor prognosis in hepatocellular carcinoma, Jpn J Clin Oncol 40, 1053-1059.

[209] Moreau, T., Baranger, K., Dade, S., Dallet-Choisy, S., Guyot, N., and Zani, M. L. (2008) Multifaceted roles of human elafin and secretory leukocyte proteinase inhibitor (SLPI), two serine protease inhibitors of the chelonianin family, Biochimie 90, 284-295.

[210] Zhang, C., Xu, Y., Gao, P., Lu, J., Li, X., and Liu, D. (2015) Down-regulation of carboxylesterases 1 and 2 plays an important role in prodrug metabolism in immunological liver injury rats, Int Immunopharmacol 24, 153-158.

[211] Yang, J., Shi, D., Yang, D., Song, X., and Yan, B. (2007) Interleukin-6 alters the cellular responsiveness to clopidogrel, irinotecan, and oseltamivir by suppressing the expression of carboxylesterases HCE1 and HCE2, Mol Pharmacol 72, 686-694.

[212] Meyer, M. R., Schutz, A., and Maurer, H. H. (2015) Contribution of human esterases to the metabolism of selected drugs of abuse, Toxicol Lett 232, 159-166.

[213] McCormack, N., Molloy, E. L., and O'Dea, S. (2013) Bone morphogenetic proteins enhance an epithelial-mesenchymal transition in normal airway epithelial cells during restitution of a disrupted epithelium, Respir Res 14, 36.

[214] Myllarniemi, M., Lindholm, P., Ryynanen, M. J., Kliment, C. R., Salmenkivi, K., Keski-Oja, J., Kinnula, V. L., Oury, T. D., and Koli, K. (2008) Gremlin-mediated decrease in bone morphogenetic protein signaling promotes pulmonary fibrosis, Am J Respir Crit Care Med 177, 321-329.

[215] Lu, M. M., Yang, H., Zhang, L., Shu, W., Blair, D. G., and Morrisey, E. E. (2001) The bone morphogenic protein antagonist gremlin regulates proximal-distal patterning of the lung, Dev Dyn 222, 667-680.

[216] Sato, Y., Hatta, M., Karim, M. F., Sawa, T., Wei, F. Y., Sato, S., Magnuson, M. A., Gonzalez, F. J., Tomizawa, K., Akaike, T., Yoshizawa, T., and Yamagata, K. (2012) Anks4b, a novel target of HNF4alpha protein, interacts with GRP78 protein and regulates endoplasmic reticulum stress-induced apoptosis in pancreatic beta-cells, J Biol Chem 287, 2323623245.

[217] Trak-Smayra, V., Dargere, D., Noun, R., Albuquerque, M., Yaghi, C., Gannage-Yared, M. H., Bedossa, P., and Paradis, V. (2009) Serum proteomic profiling of obese patients: correlation with liver pathology and evolution after bariatric surgery, Gut 58, 825-832. 
[218] Koyanagi, M., Hijikata, M., Watashi, K., Masui, O., and Shimotohno, K. (2005) Centrosomal P4.1-associated protein is a new member of transcriptional coactivators for nuclear factor-kappaB, J Biol Chem 280, 12430-12437.

[219] Yang, S. T., Yen, C. J., Lai, C. H., Lin, Y. J., Chang, K. C., Lee, J. C., Liu, Y. W., Chang-Liao, P. Y., Hsu, L. S., Chang, W. C., Hung, W. C., Tang, T. K., Liu, Y. W., and Hung, L. Y. (2013) SUMOylated CPAP is required for IKK-mediated NF-kappaB activation and enhances HBx-induced NF-kappaB signaling in HCC, J Hepatol 58, 1157-1164.

[220] Saha, S., Aranda, E., Hayakawa, Y., Bhanja, P., Atay, S., Brodin, N. P., Li, J., Asfaha, S., Liu, L., Tailor, Y., Zhang, J., Godwin, A. K., Tome, W. A., Wang, T. C., Guha, C., and Pollard, J. W. (2016) Macrophage-derived extracellular vesicle-packaged WNTs rescue intestinal stem cells and enhance survival after radiation injury, Nat Commun 7, 13096.

[221] Ledo, N., Ko, Y. A., Park, A. S., Kang, H. M., Han, S. Y., Choi, P., and Susztak, K. (2015) Functional genomic annotation of genetic risk loci highlights inflammation and epithelial biology networks in CKD, J Am Soc Nephrol 26, 692-714.

[222] Katoh, Y., and Katoh, M. (2006) Comparative genomics on HHIP family orthologs, Int J Mol Med 17, 391-395.

[223] Lopez-Mejias, R., Corrales, A., Vicente, E., Robustillo-Villarino, M., Gonzalez-Juanatey, C., Llorca, J., Genre, F., Remuzgo-Martinez, S., Dierssen-Sotos, T., Miranda-Filloy, J. A., Huaranga, M. A., Pina, T., Blanco, R., Alegre-Sancho, J. J., Raya, E., Mijares, V., Ubilla, B., Ferraz-Amaro, I., Gomez-Vaquero, C., Balsa, A., Lopez-Longo, F. J., Carreira, P., GonzalezAlvaro, I., Ocejo-Vinyals, J. G., Rodriguez-Rodriguez, L., Fernandez-Gutierrez, B., Castaneda, S., Martin, J., and Gonzalez-Gay, M. A. (2017) Influence of coronary artery disease and subclinical atherosclerosis related polymorphisms on the risk of atherosclerosis in rheumatoid arthritis, Sci Rep 7, 40303.

[224] Sasahira, T., Kurihara, M., Nishiguchi, Y., Fujiwara, R., Kirita, T., and Kuniyasu, H. (2016) NEDD 4 binding protein 2-like 1 promotes cancer cell invasion in oral squamous cell carcinoma, Virchows Arch 469, 163-172.

[225] Cohen, D. E. (2013) New players on the metabolic stage: How do you like Them Acots?, Adipocyte 2, 3-6.

[226] Xin, X., Mains, R. E., and Eipper, B. A. (2004) Monooxygenase X, a member of the copperdependent monooxygenase family localized to the endoplasmic reticulum, $J \mathrm{Biol}$ Chem 279, 48159-48167.

[227] Jee, S., Hwang, D., Seo, S., Kim, Y., Kim, C., Kim, B., Shim, S., Lee, S., Sin, J., Bae, C., Lee, B., Jang, M., Kim, M., Yim, S., Jang, I., Cho, J., and Chae, K. (2007) Microarray analysis of insulin-regulated gene expression in the liver: the use of transgenic mice co-expressing insulin-siRNA and human IDE as an animal model, Int J Mol Med 20, 829-835.

[228] Fang, T., Cui, M., Sun, J., Ge, C., Zhao, F., Zhang, L., Tian, H., Zhang, L., Chen, T., Jiang, G., Xie, H., Cui, Y., Yao, M., Li, H., and Li, J. (2015) Orosomucoid 2 inhibits tumor metastasis and is upregulated by CCAAT/enhancer binding protein beta in hepatocellular carcinomas, Oncotarget 6, 16106-16119.

[229] de Baaij, J. H., Arjona, F. J., van den Brand, M., Lavrijsen, M., Lameris, A. L., Bindels, R. J., and Hoenderop, J. G. (2016) Identification of SLC41A3 as a novel player in magnesium homeostasis, Sci Rep 6, 28565.

[230] de Baaij, J. H. (2015) The art of magnesium transport, Magnes Res 28, 85-91.

[231] Rowland, A., Miners, J. O., and Mackenzie, P. I. (2013) The UDP-glucuronosyltransferases: their role in drug metabolism and detoxification, Int J Biochem Cell Biol 45, 1121-1132. 
[232] Jo, M., Kim, J. H., Song, G. J., Seo, M., Hwang, E. M., and Suk, K. (2017) Astrocytic Orosomucoid-2 Modulates Microglial Activation and Neuroinflammation, J Neurosci 37, 2878-2894.

[233] Li, J. Y., Paragas, N., Ned, R. M., Qiu, A., Viltard, M., Leete, T., Drexler, I. R., Chen, X., SannaCherchi, S., Mohammed, F., Williams, D., Lin, C. S., Schmidt-Ott, K. M., Andrews, N. C., and Barasch, J. (2009) Scara5 is a ferritin receptor mediating non-transferrin iron delivery, Dev Cell 16, 35-46.

[234] Xu, Z., Hong, Z., Ma, M., Liu, X., Chen, L., Zheng, C., Xi, X., and Shao, J. (2016) Rock2 promotes RCC proliferation by decreasing SCARA5 expression through betacatenin/TCF4 signaling, Biochem Biophys Res Commun 480, 586-593.

[235] Depienne, C., Nava, C., Keren, B., Heide, S., Rastetter, A., Passemard, S., ChantotBastaraud, S., Moutard, M. L., Agrawal, P. B., VanNoy, G., Stoler, J. M., Amor, D. J., Billette de Villemeur, T., Doummar, D., Alby, C., Cormier-Daire, V., Garel, C., Marzin, P., Scheidecker, S., de Saint-Martin, A., Hirsch, E., Korff, C., Bottani, A., Faivre, L., Verloes, A., Orzechowski, C., Burglen, L., Leheup, B., Roume, J., Andrieux, J., Sheth, F., Datar, C., Parker, M. J., Pasquier, L., Odent, S., Naudion, S., Delrue, M. A., Le Caignec, C., Vincent, M., Isidor, B., Renaldo, F., Stewart, F., Toutain, A., Koehler, U., Hackl, B., von Stulpnagel, C., Kluger, G., Moller, R. S., Pal, D., Jonson, T., Soller, M., Verbeek, N. E., van Haelst, M. M., de Kovel, C., Koeleman, B., Monroe, G., van Haaften, G., Study, D. D. D., Attie-Bitach, T., Boutaud, L., Heron, D., and Mignot, C. (2017) Genetic and phenotypic dissection of $1 q 43 q 44$ microdeletion syndrome and neurodevelopmental phenotypes associated with mutations in ZBTB18 and HNRNPU, Hum Genet 136, 463-479.

[236] Rodriguez-Agudo, D., Ren, S., Hylemon, P. B., Montanez, R., Redford, K., Natarajan, R., Medina, M. A., Gil, G., and Pandak, W. M. (2006) Localization of StarD5 cholesterol binding protein, J Lipid Res 47, 1168-1175.

[237] Letourneau, D., Lorin, A., Lefebvre, A., Frappier, V., Gaudreault, F., Najmanovich, R., Lavigne, P., and LeHoux, J. G. (2012) StAR-related lipid transfer domain protein 5 binds primary bile acids, J Lipid Res 53, 2677-2689.

[238] Letourneau, D., Lefebvre, A., Lavigne, P., and LeHoux, J. G. (2013) STARD5 specific ligand binding: comparison with STARD1 and STARD4 subfamilies, Mol Cell Endocrinol 371, 20 25.

[239] Letourneau, D., Lorin, A., Lefebvre, A., Cabana, J., Lavigne, P., and LeHoux, J. G. (2013) Thermodynamic and solution state NMR characterization of the binding of secondary and conjugated bile acids to STARD5, Biochim Biophys Acta 1831, 1589-1599.

[240] Rodriguez-Agudo, D., Calderon-Dominguez, M., Medina, M. A., Ren, S., Gil, G., and Pandak, W. M. (2012) ER stress increases StarD5 expression by stabilizing its mRNA and leads to relocalization of its protein from the nucleus to the membranes, $J$ Lipid Res 53, 27082715.

[241] Sekine, S., Ogawa, R., Ojima, H., and Kanai, Y. (2011) Overexpression of alpha-methylacylCoA racemase is associated with CTNNB1 mutations in hepatocellular carcinomas, Histopathology 58, 712-719.

[242] Zha, S., and Isaacs, W. B. (2005) A nonclassic CCAAT enhancer element binding protein binding site contributes to alpha-methylacyl-CoA racemase expression in prostate cancer, Mol Cancer Res 3, 110-118.

[243] Dolis, D., de Kroon, A. I., and de Kruijff, B. (1996) Transmembrane movement of phosphatidylcholine in mitochondrial outer membrane vesicles, J Biol Chem 271, 1187911883. 
[244] Kang, H. W., Niepel, M. W., Han, S., Kawano, Y., and Cohen, D. E. (2012) Thioesterase superfamily member 2/acyl-CoA thioesterase 13 (Them2/Acot13) regulates hepatic lipid and glucose metabolism, FASEB J 26, 2209-2221.

[245] Kawano, Y., Ersoy, B. A., Li, Y., Nishiumi, S., Yoshida, M., and Cohen, D. E. (2014) Thioesterase superfamily member 2 (Them2) and phosphatidylcholine transfer protein (PC-TP) interact to promote fatty acid oxidation and control glucose utilization, Mol Cell Biol 34, 2396-2408.

[246] Wang, W. J., Baez, J. M., Maurer, R., Dansky, H. M., and Cohen, D. E. (2006) Homozygous disruption of Pctp modulates atherosclerosis in apolipoprotein E-deficient mice, J Lipid Res 47, 2400-2407.

[247] Wu, M. K., and Cohen, D. E. (2005) Phosphatidylcholine transfer protein regulates size and hepatic uptake of high-density lipoproteins, Am J Physiol Gastrointest Liver Physiol 289, G1067-1074.

[248] Krisko, T. I., LeClair, K. B., and Cohen, D. E. (2017) Genetic ablation of phosphatidylcholine transfer protein/StarD2 in ob/ob mice improves glucose tolerance without increasing energy expenditure, Metabolism 68, 145-149.

[249] Flores, Y. N., Velazquez-Cruz, R., Ramirez, P., Banuelos, M., Zhang, Z. F., Yee, H. F., Jr., Chang, S. C., Canizales-Quinteros, S., Quiterio, M., Cabrera-Alvarez, G., Patino, N., and Salmeron, J. (2016) Association between PNPLA3 (rs738409), LYPLAL1 (rs12137855), PPP1R3B (rs4240624), GCKR (rs780094), and elevated transaminase levels in overweight/obese Mexican adults, Mol Biol Rep 43, 1359-1369.

[250] Leon-Mimila, P., Vega-Badillo, J., Gutierrez-Vidal, R., Villamil-Ramirez, H., Villareal-Molina, T., Larrieta-Carrasco, E., Lopez-Contreras, B. E., Kauffer, L. R., Maldonado-Pintado, D. G., Mendez-Sanchez, N., Tovar, A. R., Hernandez-Pando, R., Velazquez-Cruz, R., CamposPerez, F., Aguilar-Salinas, C. A., and Canizales-Quinteros, S. (2015) A genetic risk score is associated with hepatic triglyceride content and non-alcoholic steatohepatitis in Mexicans with morbid obesity, Exp Mol Pathol 98, 178-183.

[251] Agius, L. (2008) Glucokinase and molecular aspects of liver glycogen metabolism, Biochem J 414, 1-18.

[252] ljssennagger, N., Janssen, A. W. F., Milona, A., Ramos Pittol, J. M., Hollman, D. A. A., Mokry, M., Betzel, B., Berends, F. J., Janssen, I. M., van Mil, S. W. C., and Kersten, S. (2016) Gene expression profiling in human precision cut liver slices in response to the FXR agonist obeticholic acid, J Hepatol 64, 1158-1166.

[253] Atmaca, M., Gulhan, B., Korkmaz, E., Inozu, M., Soylemezoglu, O., Candan, C., Bayazit, A. K., Elmaci, A. M., Parmaksiz, G., Duzova, A., Besbas, N., Topaloglu, R., and Ozaltin, F. (2017) Follow-up results of patients with ADCK4 mutations and the efficacy of CoQ10 treatment, Pediatr Nephrol 32, 1369-1375.

[254] Tasic, V., Gucev, Z., and Polenakovic, M. (2015) Steroid Resistant Nephrotic SyndromeGenetic Consideration, Pril (Makedon Akad Nauk Umet Odd Med Nauki) 36, 5-12.

[255] Ashraf, S., Gee, H. Y., Woerner, S., Xie, L. X., Vega-Warner, V., Lovric, S., Fang, H., Song, X., Cattran, D. C., Avila-Casado, C., Paterson, A. D., Nitschke, P., Bole-Feysot, C., Cochat, P., Esteve-Rudd, J., Haberberger, B., Allen, S. J., Zhou, W., Airik, R., Otto, E. A., Barua, M., AlHamed, M. H., Kari, J. A., Evans, J., Bierzynska, A., Saleem, M. A., Bockenhauer, D., Kleta, R., El Desoky, S., Hacihamdioglu, D. O., Gok, F., Washburn, J., Wiggins, R. C., Choi, M., Lifton, R. P., Levy, S., Han, Z., Salviati, L., Prokisch, H., Williams, D. S., Pollak, M., Clarke, C. F., Pei, Y., Antignac, C., and Hildebrandt, F. (2013) ADCK4 mutations promote steroidresistant nephrotic syndrome through CoQ10 biosynthesis disruption, J Clin Invest 123, 5179-5189. 
[256] Hu, P., Zhang, M., and Napoli, J. L. (2007) Ontogeny of rdh9 (Crad3) expression: ablation causes changes in retinoid and steroid metabolizing enzymes, but RXR and androgen signaling seem normal, Biochim Biophys Acta 1770, 694-705.

[257] Yamamoto, K., Gandin, V., Sasaki, M., McCracken, S., Li, W., Silvester, J. L., Elia, A. J., Wang, F., Wakutani, Y., Alexandrova, R., Oo, Y. D., Mullen, P. J., Inoue, S., Itsumi, M., Lapin, V., Haight, J., Wakeham, A., Shahinian, A., Ikura, M., Topisirovic, I., Sonenberg, N., and Mak, T. W. (2014) Largen: a molecular regulator of mammalian cell size control, Mol Cell 53, 904-915.

[258] Lunning, M. A., and Green, M. R. (2015) Mutation of chromatin modifiers; an emerging hallmark of germinal center B-cell lymphomas, Blood Cancer J 5, e361.

[259] Lee, J. H., Kang, Y. E., Chang, J. Y., Park, K. C., Kim, H. W., Kim, J. T., Kim, H. J., Yi, H. S., Shong, M., Chung, H. K., and Kim, K. S. (2016) An engineered FGF21 variant, LY2405319, can prevent non-alcoholic steatohepatitis by enhancing hepatic mitochondrial function, Am J Transl Res 8, 4750-4763.

[260] He, L., Deng, L., Zhang, Q., Guo, J., Zhou, J., Song, W., and Yuan, F. (2017) Diagnostic Value of CK-18, FGF-21, and Related Biomarker Panel in Nonalcoholic Fatty Liver Disease: A Systematic Review and Meta-Analysis, Biomed Res Int 2017, 9729107.

[261] Yang, M., Xu, D., Liu, Y., Guo, X., Li, W., Guo, C., Zhang, H., Gao, Y., Mao, Y., and Zhao, J. (2015) Combined Serum Biomarkers in Non-Invasive Diagnosis of Non-Alcoholic Steatohepatitis, PLoS One 10, e0131664.

[262] Liu, X., Zhang, P., Martin, R. C., Cui, G., Wang, G., Tan, Y., Cai, L., Lv, G., and Li, Y. (2016) Lack of fibroblast growth factor 21 accelerates metabolic liver injury characterized by steatohepatities in mice, Am J Cancer Res 6, 1011-1025.

[263] Ersoy Tunali, N., Marobbio, C. M., Tiryakioglu, N. O., Punzi, G., Saygili, S. K., Onal, H., and Palmieri, F. (2014) A novel mutation in the SLC25A15 gene in a Turkish patient with $\mathrm{HHH}$ syndrome: functional analysis of the mutant protein, Mol Genet Metab 112, 25-29.

[264] Wang, J. F., and Chou, K. C. (2012) Insights into the mutation-induced HHH syndrome from modeling human mitochondrial ornithine transporter-1, PLoS One 7, e31048.

[265] Rachidi, M., Delezoide, A. L., Delabar, J. M., and Lopes, C. (2009) A quantitative assessment of gene expression (QAGE) reveals differential overexpression of DOPEY2, a candidate gene for mental retardation, in Down syndrome brain regions, Int J Dev Neurosci 27, 393-398.

[266] Rachidi, M., Lopes, C., Costantine, M., and Delabar, J. M. (2005) C21orf5, a new member of Dopey family involved in morphogenesis, could participate in neurological alterations and mental retardation in Down syndrome, DNA Res 12, 203-210.

[267] Swaminathan, S., Huentelman, M. J., Corneveaux, J. J., Myers, A. J., Faber, K. M., Foroud, T., Mayeux, R., Shen, L., Kim, S., Turk, M., Hardy, J., Reiman, E. M., Saykin, A. J., Alzheimer's Disease Neuroimaging, I., and Group, N.-L. N. F. S. (2012) Analysis of copy number variation in Alzheimer's disease in a cohort of clinically characterized and neuropathologically verified individuals, PLoS One 7, e50640.

[268] Swaminathan, S., Shen, L., Kim, S., Inlow, M., West, J. D., Faber, K. M., Foroud, T., Mayeux, R., Saykin, A. J., Alzheimer's Disease Neuroimaging, I., and Group, N.-L. N. F. S. (2012) Analysis of copy number variation in Alzheimer's disease: the NIALOAD/ NCRAD Family Study, Curr Alzheimer Res 9, 801-814.

[269] Stepanova, M., Hossain, N., Afendy, A., Perry, K., Goodman, Z. D., Baranova, A., and Younossi, Z. (2010) Hepatic gene expression of Caucasian and African-American patients with obesity-related non-alcoholic fatty liver disease, Obes Surg 20, 640-650. 
[270] Babelova, A., Burckhardt, B. C., Salinas-Riester, G., Pommerenke, C., Burckhardt, G., and Henjakovic, M. (2015) Next generation sequencing of sex-specific genes in the livers of obese ZSF1 rats, Genomics 106, 204-213.

[271] Li, C. Y., Renaud, H. J., Klaassen, C. D., and Cui, J. Y. (2016) Age-Specific Regulation of DrugProcessing Genes in Mouse Liver by Ligands of Xenobiotic-Sensing Transcription Factors, Drug Metab Dispos 44, 1038-1049.

[272] Chou, F. P., Chu, Y. C., Hsu, J. D., Chiang, H. C., and Wang, C. J. (2000) Specific induction of glutathione S-transferase GSTM2 subunit expression by epigallocatechin gallate in rat liver, Biochem Pharmacol 60, 643-650.

[273] Bernsmeier, C., Dill, M. T., Provenzano, A., Makowska, Z., Krol, I., Muscogiuri, G., Semela, D., Tornillo, L., Marra, F., Heim, M. H., and Duong, F. H. (2016) Hepatic Notch1 deletion predisposes to diabetes and steatosis via glucose-6-phosphatase and perilipin-5 upregulation, Lab Invest 96, 972-980.

[274] Najt, C. P., Senthivinayagam, S., Aljazi, M. B., Fader, K. A., Olenic, S. D., Brock, J. R., Lydic, T. A., Jones, A. D., and Atshaves, B. P. (2016) Liver-specific loss of Perilipin 2 alleviates dietinduced hepatic steatosis, inflammation, and fibrosis, Am J Physiol Gastrointest Liver Physiol 310, G726-738.

[275] Pawella, L. M., Hashani, M., Eiteneuer, E., Renner, M., Bartenschlager, R., Schirmacher, P., and Straub, B. K. (2014) Perilipin discerns chronic from acute hepatocellular steatosis, J Hepatol 60, 633-642.

[276] Fujii, H., Ikura, Y., Arimoto, J., Sugioka, K., lezzoni, J. C., Park, S. H., Naruko, T., Itabe, H., Kawada, N., Caldwell, S. H., and Ueda, M. (2009) Expression of perilipin and adipophilin in nonalcoholic fatty liver disease; relevance to oxidative injury and hepatocyte ballooning, J Atheroscler Thromb 16, 893-901.

[277] Rossmanith, W. (2011) Localization of human RNase Z isoforms: dual nuclear/mitochondrial targeting of the ELAC2 gene product by alternative translation initiation, PLoS One 6, e19152.

[278] Graff, E. C., Fang, H., Wanders, D., and Judd, R. L. (2016) Anti-inflammatory effects of the hydroxycarboxylic acid receptor 2, Metabolism 65, 102-113.

[279] Liu, C., Kuei, C., Zhu, J., Yu, J., Zhang, L., Shih, A., Mirzadegan, T., Shelton, J., Sutton, S., Connelly, M. A., Lee, G., Carruthers, N., Wu, J., and Lovenberg, T. W. (2012) 3,5Dihydroxybenzoic acid, a specific agonist for hydroxycarboxylic acid 1, inhibits lipolysis in adipocytes, J Pharmacol Exp Ther 341, 794-801.

[280] Morland, C., Lauritzen, K. H., Puchades, M., Holm-Hansen, S., Andersson, K., Gjedde, A., Attramadal, H., Storm-Mathisen, J., and Bergersen, L. H. (2015) The lactate receptor, Gprotein-coupled receptor 81/hydroxycarboxylic acid receptor 1: Expression and action in brain, J Neurosci Res 93, 1045-1055.

[281] Feingold, K. R., Moser, A., Shigenaga, J. K., and Grunfeld, C. (2014) Inflammation stimulates niacin receptor (GPR109A/HCA2) expression in adipose tissue and macrophages, J Lipid Res 55, 2501-2508.

[282] El-Zaatari, M., and Kao, J. Y. (2017) Role of Dietary Metabolites in Regulating the Host Immune Response in Gastrointestinal Disease, Front Immunol 8, 51.

[283] Gonzalez Malagon, S. G., Melidoni, A. N., Hernandez, D., Omar, B. A., Houseman, L., Veeravalli, S., Scott, F., Varshavi, D., Everett, J., Tsuchiya, Y., Timms, J. F., Phillips, I. R., and Shephard, E. A. (2015) The phenotype of a knockout mouse identifies flavincontaining monooxygenase 5 (FMO5) as a regulator of metabolic ageing, Biochem Pharmacol 96, 267-277. 
[284] Solda, G., Caccia, S., Robusto, M., Chiereghin, C., Castorina, P., Ambrosetti, U., Duga, S., and Asselta, R. (2016) First independent replication of the involvement of LARS2 in Perrault syndrome by whole-exome sequencing of an Italian family, J Hum Genet 61, 295-300.

[285] Newman, W. G., Friedman, T. B., and Conway, G. S. (1993) Perrault Syndrome, In GeneReviews(R) (Adam, M. P., Ardinger, H. H., Pagon, R. A., Wallace, S. E., Bean, L. J. H., Mefford, H. C., Stephens, K., Amemiya, A., and Ledbetter, N., Eds.), Seattle (WA).

[286] Riley, L. G., Rudinger-Thirion, J., Schmitz-Abe, K., Thorburn, D. R., Davis, R. L., Teo, J., Arbuckle, S., Cooper, S. T., Campagna, D. R., Frugier, M., Markianos, K., Sue, C. M., Fleming, M. D., and Christodoulou, J. (2016) LARS2 Variants Associated with Hydrops, Lactic Acidosis, Sideroblastic Anemia, and Multisystem Failure, JIMD Rep 28, 49-57.

[287] Hart, L. M., Hansen, T., Rietveld, I., Dekker, J. M., Nijpels, G., Janssen, G. M., Arp, P. A., Uitterlinden, A. G., Jorgensen, T., Borch-Johnsen, K., Pols, H. A., Pedersen, O., van Duijn, C. M., Heine, R. J., and Maassen, J. A. (2005) Evidence that the mitochondrial leucyl tRNA synthetase (LARS2) gene represents a novel type 2 diabetes susceptibility gene, Diabetes 54, 1892-1895.

[288] Reiling, E., Jafar-Mohammadi, B., van 't Riet, E., Weedon, M. N., van Vliet-Ostaptchouk, J. V., Hansen, T., Saxena, R., van Haeften, T. W., Arp, P. A., Das, S., Nijpels, G., Groenewoud, M. J., van Hove, E. C., Uitterlinden, A. G., Smit, J. W., Morris, A. D., Doney, A. S., Palmer, C. N., Guiducci, C., Hattersley, A. T., Frayling, T. M., Pedersen, O., Slagboom, P. E., Altshuler, D. M., Groop, L., Romijn, J. A., Maassen, J. A., Hofker, M. H., Dekker, J. M., McCarthy, M. I., and t Hart, L. M. (2010) Genetic association analysis of LARS2 with type 2 diabetes, Diabetologia 53, 103-110.

[289] Sutrisna, E. (2016) The Impact of CYP1A2 and CYP2E1 Genes Polymorphism on Theophylline Response, J Clin Diagn Res 10, FE01-FE03.

[290] Li, H., Clarke, J. D., Dzierlenga, A. L., Bear, J., Goedken, M. J., and Cherrington, N. J. (2017) In vivo cytochrome $\mathrm{P} 450$ activity alterations in diabetic nonalcoholic steatohepatitis mice, J Biochem Mol Toxicol 31.

[291] Fisher, C. D., Lickteig, A. J., Augustine, L. M., Ranger-Moore, J., Jackson, J. P., Ferguson, S. S., and Cherrington, N. J. (2009) Hepatic cytochrome P450 enzyme alterations in humans with progressive stages of nonalcoholic fatty liver disease, Drug Metab Dispos 37, 20872094.

[292] Al Alam, D., Danopoulos, S., Schall, K., Sala, F. G., Almohazey, D., Fernandez, G. E., Georgia, S., Frey, M. R., Ford, H. R., Grikscheit, T., and Bellusci, S. (2015) Fibroblast growth factor 10 alters the balance between goblet and Paneth cells in the adult mouse small intestine, Am J Physiol Gastrointest Liver Physiol 308, G678-690.

[293] Lili, L. N., Farkas, A. E., Gerner-Smidt, C., Overgaard, C. E., Moreno, C. S., Parkos, C. A., Capaldo, C. T., and Nusrat, A. (2016) Claudin-based barrier differentiation in the colonic epithelial crypt niche involves Hopx/KIf4 and Tcf7/2/Hnf4-alpha cascades, Tissue Barriers 4, e1214038.

[294] Kelly, E. E., Giordano, F., Horgan, C. P., Jollivet, F., Raposo, G., and McCaffrey, M. W. (2012) Rab30 is required for the morphological integrity of the Golgi apparatus, Biol Cell 104, 84-101.

[295] Li, Q., Wang, J., Wan, Y., and Chen, D. (2016) Depletion of Rab32 decreases intracellular lipid accumulation and induces lipolysis through enhancing ATGL expression in hepatocytes, Biochem Biophys Res Commun 471, 492-496.

[296] Klein, C. (2009) Congenital neutropenia, Hematology Am Soc Hematol Educ Program, 344350. 
[297] Reissmann, M., and Ludwig, A. (2013) Pleiotropic effects of coat colour-associated mutations in humans, mice and other mammals, Semin Cell Dev Biol 24, 576-586.

[298] Brandt-Bohne, U., Keene, D. R., White, F. A., and Koch, M. (2007) MEGF9: a novel transmembrane protein with a strong and developmentally regulated expression in the nervous system, Biochem J 401, 447-457.

[299] Lopez-Corral, L., Corchete, L. A., Sarasquete, M. E., Mateos, M. V., Garcia-Sanz, R., Ferminan, E., Lahuerta, J. J., Blade, J., Oriol, A., Teruel, A. I., Martino, M. L., Hernandez, J., Hernandez-Rivas, J. M., Burguillo, F. J., San Miguel, J. F., and Gutierrez, N. C. (2014) Transcriptome analysis reveals molecular profiles associated with evolving steps of monoclonal gammopathies, Haematologica 99, 1365-1372.

[300] Wong, S., Tan, K., Carey, K. T., Fukushima, A., Tiganis, T., and Cole, T. J. (2010) Glucocorticoids stimulate hepatic and renal catecholamine inactivation by direct rapid induction of the dopamine sulfotransferase Sult1d1, Endocrinology 151, 185-194.

[301] Teufel, A., Becker, D., Weber, S. N., Dooley, S., Breitkopf-Heinlein, K., Maass, T., Hochrath, K., Krupp, M., Marquardt, J. U., Kolb, M., Korn, B., Niehrs, C., Zimmermann, T., Godoy, P., Galle, P. R., and Lammert, F. (2012) Identification of RARRES1 as a core regulator in liver fibrosis, J Mol Med (Berl) 90, 1439-1447.

[302] Nakayama, K., Fukamachi, S., Kimura, H., Koda, Y., Soemantri, A., and Ishida, T. (2002) Distinctive distribution of AIM1 polymorphism among major human populations with different skin color, J Hum Genet 47, 92-94.

[303] Du, J., and Fisher, D. E. (2002) Identification of Aim-1 as the underwhite mouse mutant and its transcriptional regulation by MITF, J Biol Chem 277, 402-406.

[304] Teichmann, U., Ray, M. E., Ellison, J., Graham, C., Wistow, G., Meltzer, P. S., Trent, J. M., and Pavan, W. J. (1998) Cloning and tissue expression of the mouse ortholog of AIM1, a betagamma-crystallin superfamily member, Mamm Genome 9, 715-720.

[305] Qi, Y., Yu, Y., Wu, Y., Wang, S., Yu, Q., Shi, J., Xu, Z., Zhang, Q., Fu, Y., Fu, Y., and Kou, C. (2015) Genetic Variants in Six-Transmembrane Epithelial Antigen of Prostate 4 Increase Risk of Developing Metabolic Syndrome in a Han Chinese Population, Genet Test Mol Biomarkers 19, 666-672.

[306] Zhang, W., Tang, M., Zhong, M., Wang, Z., Shang, Y., Gong, H., Zhang, Y., and Zhang, W. (2013) Association of the six transmembrane protein of prostate 2 gene polymorphisms with metabolic syndrome in Han Chinese population, Diabetes Metab Syndr 7, 138-142.

[307] Miot, A., Maimaitiming, S., Emery, N., Bellili, N., Roussel, R., Tichet, J., Velho, G., Balkau, B., Marre, M., Fumeron, F., and Group, D. S. (2010) Genetic variability at the six transmembrane protein of prostate 2 locus and the metabolic syndrome: the data from an epidemiological study on the Insulin Resistance Syndrome (DESIR) study, J Clin Endocrinol Metab 95, 2942-2947.

[308] Ramadoss, P., Chiappini, F., Bilban, M., and Hollenberg, A. N. (2010) Regulation of hepatic six transmembrane epithelial antigen of prostate 4 (STEAP4) expression by STAT3 and CCAAT/enhancer-binding protein alpha, J Biol Chem 285, 16453-16466.

[309] Sparna, T., Retey, J., Schmich, K., Albrecht, U., Naumann, K., Gretz, N., Fischer, H. P., Bode, J. G., and Merfort, I. (2010) Genome-wide comparison between IL-17 and combined TNF-alpha/IL-17 induced genes in primary murine hepatocytes, BMC Genomics 11, 226.

[310] Anwer, M. S., and Stieger, B. (2014) Sodium-dependent bile salt transporters of the SLC10A transporter family: more than solute transporters, Pflugers Arch 466, 77-89.

[311] Shih, D. Q., Bussen, M., Sehayek, E., Ananthanarayanan, M., Shneider, B. L., Suchy, F. J., Shefer, S., Bollileni, J. S., Gonzalez, F. J., Breslow, J. L., and Stoffel, M. (2001) Hepatocyte 
nuclear factor-1alpha is an essential regulator of bile acid and plasma cholesterol metabolism, Nat Genet 27, 375-382.

[312] Chen, F., Ma, L., Al-Ansari, N., and Shneider, B. (2001) The role of AP-1 in the transcriptional regulation of the rat apical sodium-dependent bile acid transporter, $J$ Biol Chem 276, 38703-38714.

[313] Katoh, Y., Takemori, H., Min, L., Muraoka, M., Doi, J., Horike, N., and Okamoto, M. (2004) Salt-inducible kinase-1 represses cAMP response element-binding protein activity both in the nucleus and in the cytoplasm, Eur J Biochem 271, 4307-4319.

[314] Okamoto, M., Takemori, H., and Katoh, Y. (2004) Salt-inducible kinase in steroidogenesis and adipogenesis, Trends Endocrinol Metab 15, 21-26.

[315] Guillemot, L., Schneider, Y., Brun, P., Castagliuolo, I., Pizzuti, D., Martines, D., Jond, L., Bongiovanni, M., and Citi, S. (2012) Cingulin is dispensable for epithelial barrier function and tight junction structure, and plays a role in the control of claudin-2 expression and response to duodenal mucosa injury, J Cell Sci 125, 5005-5014.

[316] Citi, S., Guerrera, D., Spadaro, D., and Shah, J. (2014) Epithelial junctions and Rho family GTPases: the zonular signalosome, Small GTPases 5, 1-15.

[317] Porat-Shliom, N., Tietgens, A. J., Van Itallie, C. M., Vitale-Cross, L., Jarnik, M., Harding, O. J., Anderson, J. M., Gutkind, J. S., Weigert, R., and Arias, I. M. (2016) Liver kinase B1 regulates hepatocellular tight junction distribution and function in vivo, Hepatology 64, 1317-1329.

[318] Walpen, T., Kalus, I., Schwaller, J., Peier, M. A., Battegay, E. J., and Humar, R. (2012) Nuclear PIM1 confers resistance to rapamycin-impaired endothelial proliferation, Biochem Biophys Res Commun 429, 24-30.

[319] Leung, C. O., Wong, C. C., Fan, D. N., Kai, A. K., Tung, E. K., Xu, I. M., Ng, I. O., and Lo, R. C. (2015) PIM1 regulates glycolysis and promotes tumor progression in hepatocellular carcinoma, Oncotarget 6, 10880-10892.

[320] Braso-Maristany, F., Filosto, S., Catchpole, S., Marlow, R., Quist, J., Francesch-Domenech, E., Plumb, D. A., Zakka, L., Gazinska, P., Liccardi, G., Meier, P., Gris-Oliver, A., Cheang, M. C., Perdrix-Rosell, A., Shafat, M., Noel, E., Patel, N., McEachern, K., Scaltriti, M., Castel, P., Noor, F., Buus, R., Mathew, S., Watkins, J., Serra, V., Marra, P., Grigoriadis, A., and Tutt, A. N. (2016) PIM1 kinase regulates cell death, tumor growth and chemotherapy response in triple-negative breast cancer, Nat Med 22, 1303-1313.

[321] Gu, J. J., Wang, Z., Reeves, R., and Magnuson, N. S. (2009) PIM1 phosphorylates and negatively regulates ASK1-mediated apoptosis, Oncogene 28, 4261-4271.

[322] Chiocchetti, A., Gibello, L., Carando, A., Aspesi, A., Secco, P., Garelli, E., Loreni, F., Angelini, M., Biava, A., Dahl, N., Dianzani, U., Ramenghi, U., Santoro, C., and Dianzani, I. (2005) Interactions between RPS19, mutated in Diamond-Blackfan anemia, and the PIM-1 oncoprotein, Haematologica 90, 1453-1462.

[323] Lu, S. C. (2013) Glutathione synthesis, Biochim Biophys Acta 1830, 3143-3153.

[324] Chen, Y., Yang, Y., Miller, M. L., Shen, D., Shertzer, H. G., Stringer, K. F., Wang, B., Schneider, S. N., Nebert, D. W., and Dalton, T. P. (2007) Hepatocyte-specific Gclc deletion leads to rapid onset of steatosis with mitochondrial injury and liver failure, Hepatology 45, 1118-1128.

[325] Krieger, J. R., Taylor, P., Gajadhar, A. S., Guha, A., Moran, M. F., and McGlade, C. J. (2013) Identification and selected reaction monitoring (SRM) quantification of endocytosis factors associated with Numb, Mol Cell Proteomics 12, 499-514. 
[326] Cullis, D. N., Philip, B., Baleja, J. D., and Feig, L. A. (2002) Rab11-FIP2, an adaptor protein connecting cellular components involved in internalization and recycling of epidermal growth factor receptors, J Biol Chem 277, 49158-49166.

[327] Chen, J. Y., Chou, H. C., Chen, Y. H., and Chan, H. L. (2013) High glucose-induced proteome alterations in hepatocytes and its possible relevance to diabetic liver disease, $J$ Nutr Biochem 24, 1889-1910.

[328] Royo, F., Moreno, L., Mleczko, J., Palomo, L., Gonzalez, E., Cabrera, D., Cogolludo, A., Vizcaino, F. P., van-Liempd, S., and Falcon-Perez, J. M. (2017) Hepatocyte-secreted extracellular vesicles modify blood metabolome and endothelial function by an arginase-dependent mechanism, Sci Rep 7, 42798.

[329] Chen, T. Y., Li, Y. C., Liu, Y. F., Tsai, C. M., Hsieh, Y. H., Lin, C. W., Yang, S. F., and Weng, C. J. (2011) Role of MMP14 gene polymorphisms in susceptibility and pathological development to hepatocellular carcinoma, Ann Surg Oncol 18, 2348-2356.

[330] Blaine, S. A., Ray, K. C., Branch, K. M., Robinson, P. S., Whitehead, R. H., and Means, A. L. (2009) Epidermal growth factor receptor regulates pancreatic fibrosis, Am J Physiol Gastrointest Liver Physiol 297, G434-441.

[331] Mitchell, C., Nivison, M., Jackson, L. F., Fox, R., Lee, D. C., Campbell, J. S., and Fausto, N. (2005) Heparin-binding epidermal growth factor-like growth factor links hepatocyte priming with cell cycle progression during liver regeneration, J Biol Chem 280, 25622568.

[332] Souza Pauli, L. S., Ropelle, E. C., de Souza, C. T., Cintra, D. E., da Silva, A. S., de Almeida Rodrigues, B., de Moura, L. P., Marinho, R., de Oliveira, V., Katashima, C. K., Pauli, J. R., and Ropelle, E. R. (2014) Exercise training decreases mitogen-activated protein kinase phosphatase- 3 expression and suppresses hepatic gluconeogenesis in obese mice, $J$ Physiol 592, 1325-1340.

[333] Patterson, K. I., Brummer, T., O'Brien, P. M., and Daly, R. J. (2009) Dual-specificity phosphatases: critical regulators with diverse cellular targets, Biochem J 418, 475-489.

[334] Li, C., Scott, D. A., Hatch, E., Tian, X., and Mansour, S. L. (2007) Dusp6 (Mkp3) is a negative feedback regulator of FGF-stimulated ERK signaling during mouse development, Development 134, 167-176.

[335] Jennemann, R., Rothermel, U., Wang, S., Sandhoff, R., Kaden, S., Out, R., van Berkel, T. J., Aerts, J. M., Ghauharali, K., Sticht, C., and Grone, H. J. (2010) Hepatic glycosphingolipid deficiency and liver function in mice, Hepatology 51, 1799-1809.

[336] Promrat, K., Longato, L., Wands, J. R., and de la Monte, S. M. (2011) Weight loss amelioration of non-alcoholic steatohepatitis linked to shifts in hepatic ceramide expression and serum ceramide levels, Hepatol Res 41, 754-762.

[337] Chi, X., Zhang, A., Luo, G., Xia, H., Zhu, G., Hei, Z., Liu, X., Wei, J., and Xia, Z. (2013) Knockdown of myeloid differentiation protein-2 reduces acute lung injury following orthotopic autologous liver transplantation in a rat model, Pulm Pharmacol Ther 26, 380-387.

[338] Hritz, I., Mandrekar, P., Velayudham, A., Catalano, D., Dolganiuc, A., Kodys, K., Kurt-Jones, E., and Szabo, G. (2008) The critical role of toll-like receptor (TLR) 4 in alcoholic liver disease is independent of the common TLR adapter MyD88, Hepatology 48, 1224-1231.

[339] Nguyen, T. T., Park, W. S., Park, B. O., Kim, C. Y., Oh, Y., Kim, J. M., Choi, H., Kyung, T., Kim, C. H., Lee, G., Hahn, K. M., Meyer, T., and Heo, W. D. (2016) PLEKHG3 enhances polarized cell migration by activating actin filaments at the cell front, Proc Natl Acad Sci U S A 113, 10091-10096. 
[340] Miao, H., Nickel, C. H., Cantley, L. G., Bruggeman, L. A., Bennardo, L. N., and Wang, B. (2003) EphA kinase activation regulates HGF-induced epithelial branching morphogenesis, J Cell Biol 162, 1281-1292.

[341] Alisi, A., Da Sacco, L., Bruscalupi, G., Piemonte, F., Panera, N., De Vito, R., Leoni, S., Bottazzo, G. F., Masotti, A., and Nobili, V. (2011) Mirnome analysis reveals novel molecular determinants in the pathogenesis of diet-induced nonalcoholic fatty liver disease, Lab Invest 91, 283-293.

[342] Wanninger, J., Neumeier, M., Hellerbrand, C., Schacherer, D., Bauer, S., Weiss, T. S., Huber, H., Schaffler, A., Aslanidis, C., Scholmerich, J., and Buechler, C. (2011) Lipid accumulation impairs adiponectin-mediated induction of activin A by increasing TGFbeta in primary human hepatocytes, Biochim Biophys Acta 1811, 626-633.

[343] Coulon, S., Heindryckx, F., Geerts, A., Van Steenkiste, C., Colle, I., and Van Vlierberghe, H. (2011) Angiogenesis in chronic liver disease and its complications, Liver Int 31, 146-162.

[344] Wang, Y., Xu, L. Y., Lam, K. S., Lu, G., Cooper, G. J., and Xu, A. (2006) Proteomic characterization of human serum proteins associated with the fat-derived hormone adiponectin, Proteomics 6, 3862-3870.

[345] Baggott, J. E., and MacKenzie, R. E. (2003) 5,10-methenyltetrahydrofolate cyclohydrolase, rat liver and chemically catalysed formation of 5-formyltetrahydrofolate, Biochem J 374, 773-778.

[346] Noakes, C. J., Lee, G., and Lowe, M. (2011) The PH domain proteins IPIP27A and B link OCRL1 to receptor recycling in the endocytic pathway, Mol Biol Cell 22, 606-623.

[347] Hasenfuss, S. C., Bakiri, L., Thomsen, M. K., Williams, E. G., Auwerx, J., and Wagner, E. F. (2014) Regulation of steatohepatitis and PPARgamma signaling by distinct AP-1 dimers, Cell Metab 19, 84-95.

[348] Veiga-da-Cunha, M., Tyteca, D., Stroobant, V., Courtoy, P. J., Opperdoes, F. R., and Van Schaftingen, E. (2010) Molecular identification of NAT8 as the enzyme that acetylates cysteine S-conjugates to mercapturic acids, J Biol Chem 285, 18888-18898.

[349] Grimm, F. A., Hu, D., Kania-Korwel, I., Lehmler, H. J., Ludewig, G., Hornbuckle, K. C., Duffel, M. W., Bergman, A., and Robertson, L. W. (2015) Metabolism and metabolites of polychlorinated biphenyls, Crit Rev Toxicol 45, 245-272.

[350] Wang, B., Hsu, S. H., Frankel, W., Ghoshal, K., and Jacob, S. T. (2012) Stat3-mediated activation of microRNA-23a suppresses gluconeogenesis in hepatocellular carcinoma by down-regulating glucose-6-phosphatase and peroxisome proliferator-activated receptor gamma, coactivator 1 alpha, Hepatology 56, 186-197.

[351] Oh, K. J., Han, H. S., Kim, M. J., and Koo, S. H. (2013) CREB and FoxO1: two transcription factors for the regulation of hepatic gluconeogenesis, BMB Rep 46, 567-574.

[352] Yin, M., Ikejima, K., Wheeler, M. D., Bradford, B. U., Seabra, V., Forman, D. T., Sato, N., and Thurman, R. G. (2000) Estrogen is involved in early alcohol-induced liver injury in a rat enteral feeding model, Hepatology 31, 117-123.

[353] Su, G. L., Rahemtulla, A., Thomas, P., Klein, R. D., Wang, S. C., and Nanji, A. A. (1998) CD14 and lipopolysaccharide binding protein expression in a rat model of alcoholic liver disease, Am J Pathol 152, 841-849.

[354] Brun, P., Castagliuolo, I., Floreani, A. R., Buda, A., Blasone, L., Palu, G., and Martines, D. (2006) Increased risk of NASH in patients carrying the C(-159)T polymorphism in the CD14 gene promoter region, Gut 55, 1212.

[355] Du, L., Neis, M. M., Ladd, P. A., and Keeney, D. S. (2006) Differentiation-specific factors modulate epidermal CYP1-4 gene expression in human skin in response to retinoic acid and classic aryl hydrocarbon receptor ligands, J Pharmacol Exp Ther 319, 1162-1171. 
[356] Scott, A., Weldon, S., and Taggart, C. C. (2011) SLPI and elafin: multifunctional antiproteases of the WFDC family, Biochem Soc Trans 39, 1437-1440.

[357] La Rocca, G., Anzalone, R., and Farina, F. (2009) The expression of CD68 in human umbilical cord mesenchymal stem cells: new evidences of presence in non-myeloid cell types, Scand J Immunol 70, 161-162.

[358] Kamino, H., Yamazaki, Y., Saito, K., Takizawa, D., Kakizaki, S., Moore, R., and Negishi, M. (2011) Nuclear receptor CAR-regulated expression of the FAM84A gene during the development of mouse liver tumors, Int J Oncol 38, 1511-1520.

[359] Kogelman, L. J., Fu, J., Franke, L., Greve, J. W., Hofker, M., Rensen, S. S., and Kadarmideen, H. N. (2016) Inter-Tissue Gene Co-Expression Networks between Metabolically Healthy and Unhealthy Obese Individuals, PLoS One 11, e0167519.

[360] Lee, S. J., Yoo, J. D., Choi, S. Y., and Kwon, O. S. (2014) The expression and secretion of vimentin in the progression of non-alcoholic steatohepatitis, BMB Rep 47, 457-462.

[361] Syn, W. K., Choi, S. S., Liaskou, E., Karaca, G. F., Agboola, K. M., Oo, Y. H., Mi, Z., Pereira, T. A., Zdanowicz, M., Malladi, P., Chen, Y., Moylan, C., Jung, Y., Bhattacharya, S. D., Teaberry, V., Omenetti, A., Abdelmalek, M. F., Guy, C. D., Adams, D. H., Kuo, P. C., Michelotti, G. A., Whitington, P. F., and Diehl, A. M. (2011) Osteopontin is induced by hedgehog pathway activation and promotes fibrosis progression in nonalcoholic steatohepatitis, Hepatology 53, 106-115.

[362] Singh, A. B., and Harris, R. C. (2004) Epidermal growth factor receptor activation differentially regulates claudin expression and enhances transepithelial resistance in Madin-Darby canine kidney cells, J Biol Chem 279, 3543-3552.

[363] Park, J. H., Liu, L., Kim, I. H., Kim, J. H., You, K. R., and Kim, D. G. (2005) Identification of the genes involved in enhanced fenretinide-induced apoptosis by parthenolide in human hepatoma cells, Cancer Res 65, 2804-2814.

[364] Engelhardt, C. M., Bundschu, K., Messerschmitt, M., Renne, T., Walter, U., Reinhard, M., and Schuh, K. (2004) Expression and subcellular localization of Spred proteins in mouse and human tissues, Histochem Cell Biol 122, 527-538. 


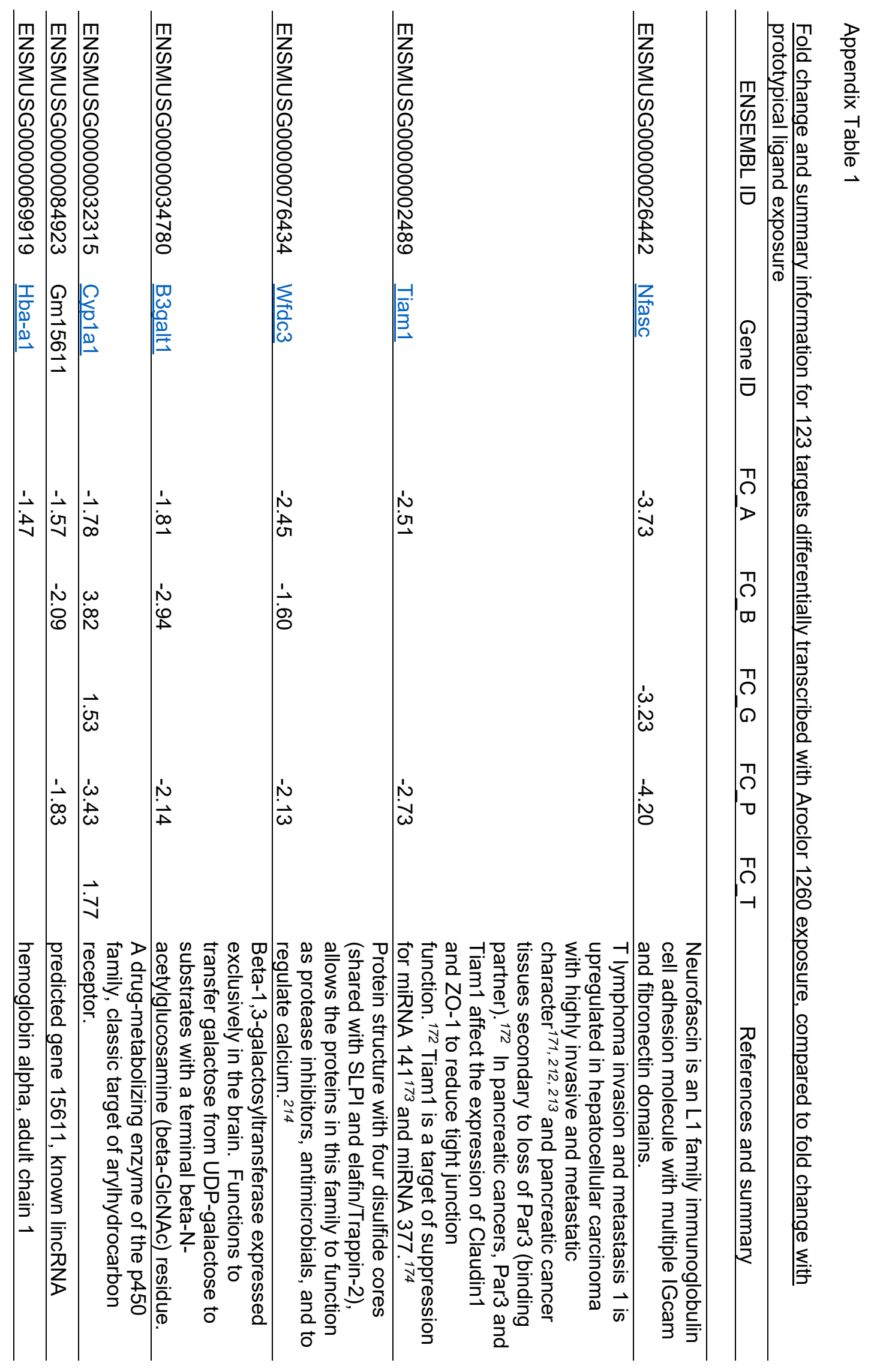




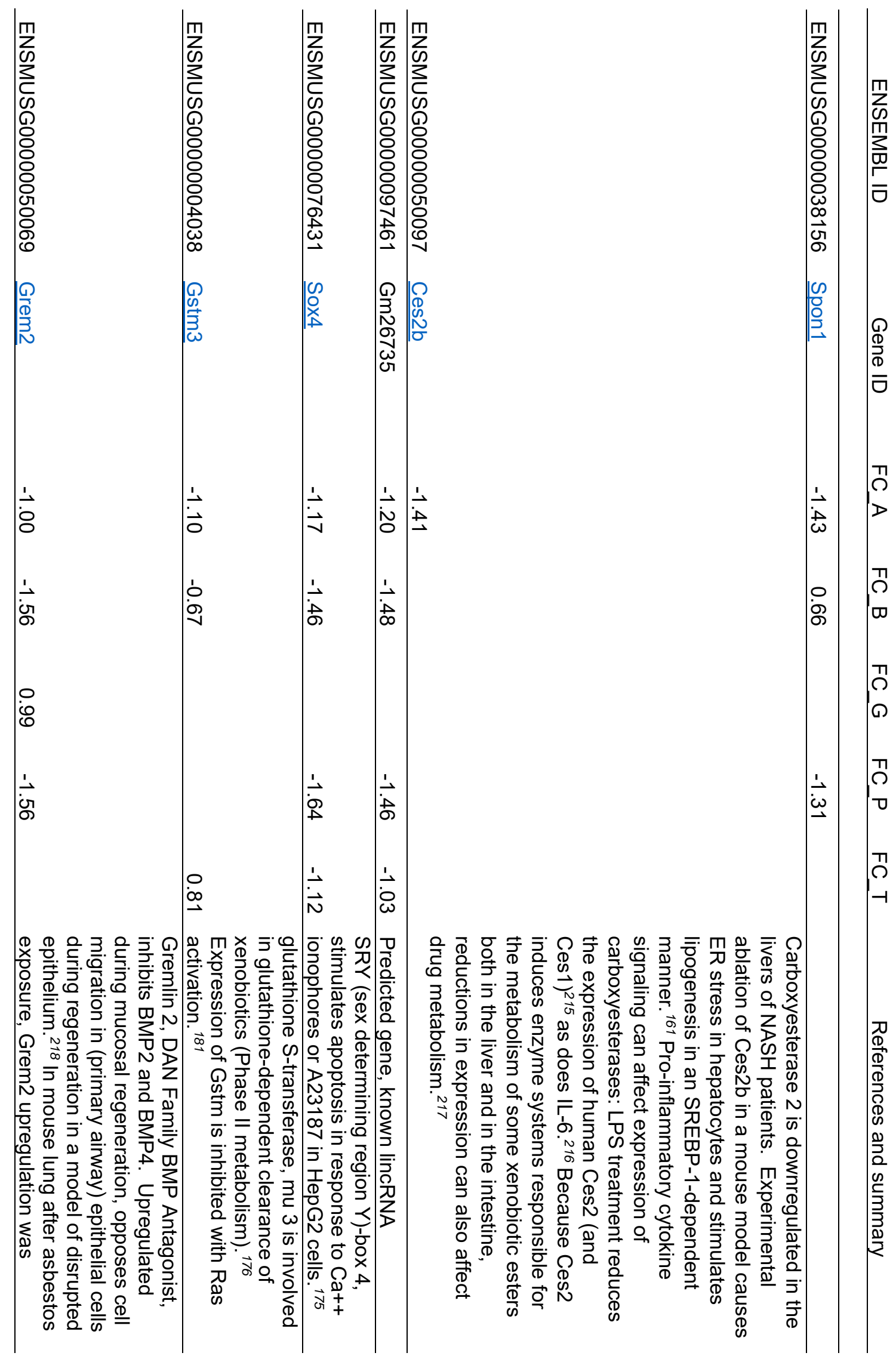




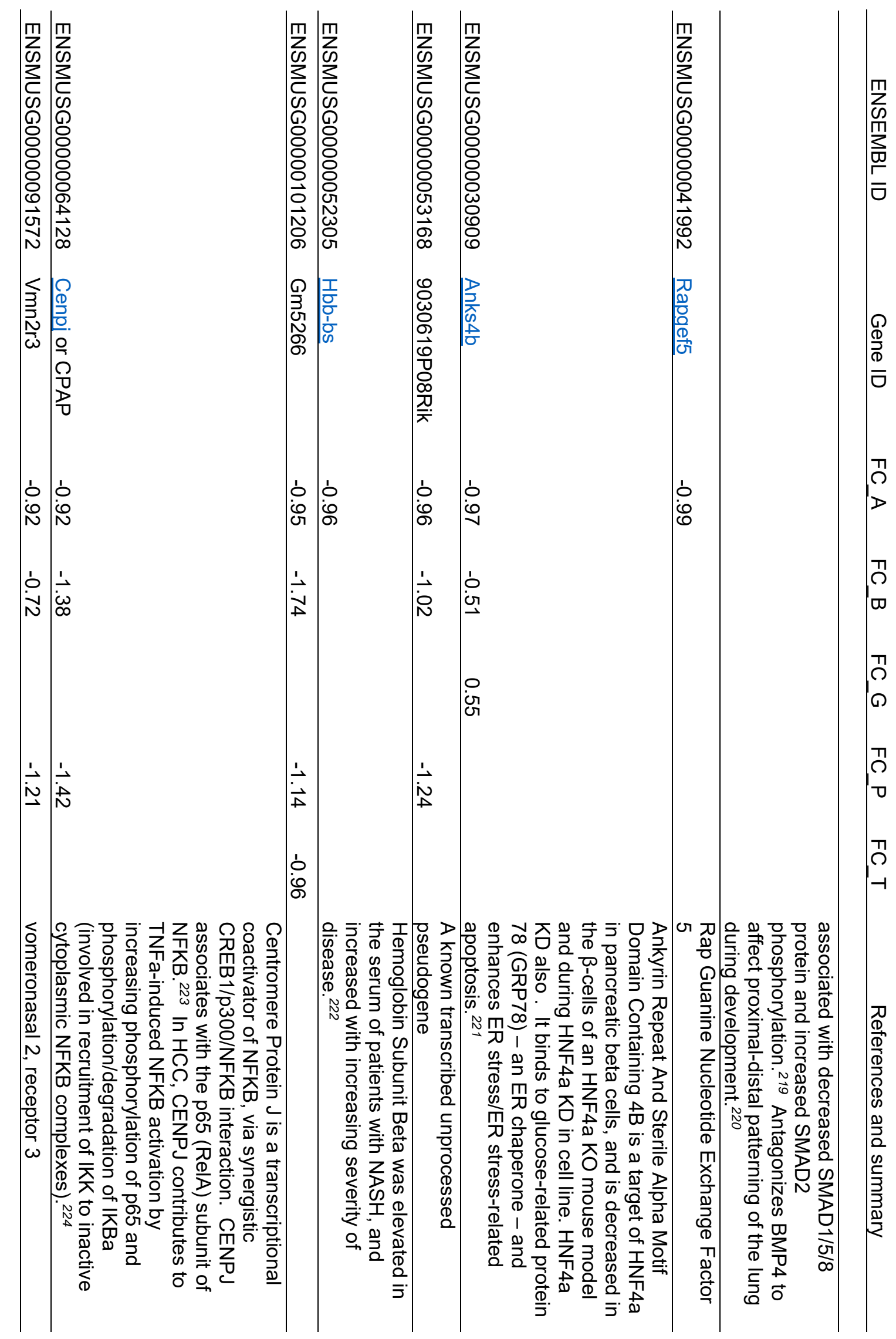




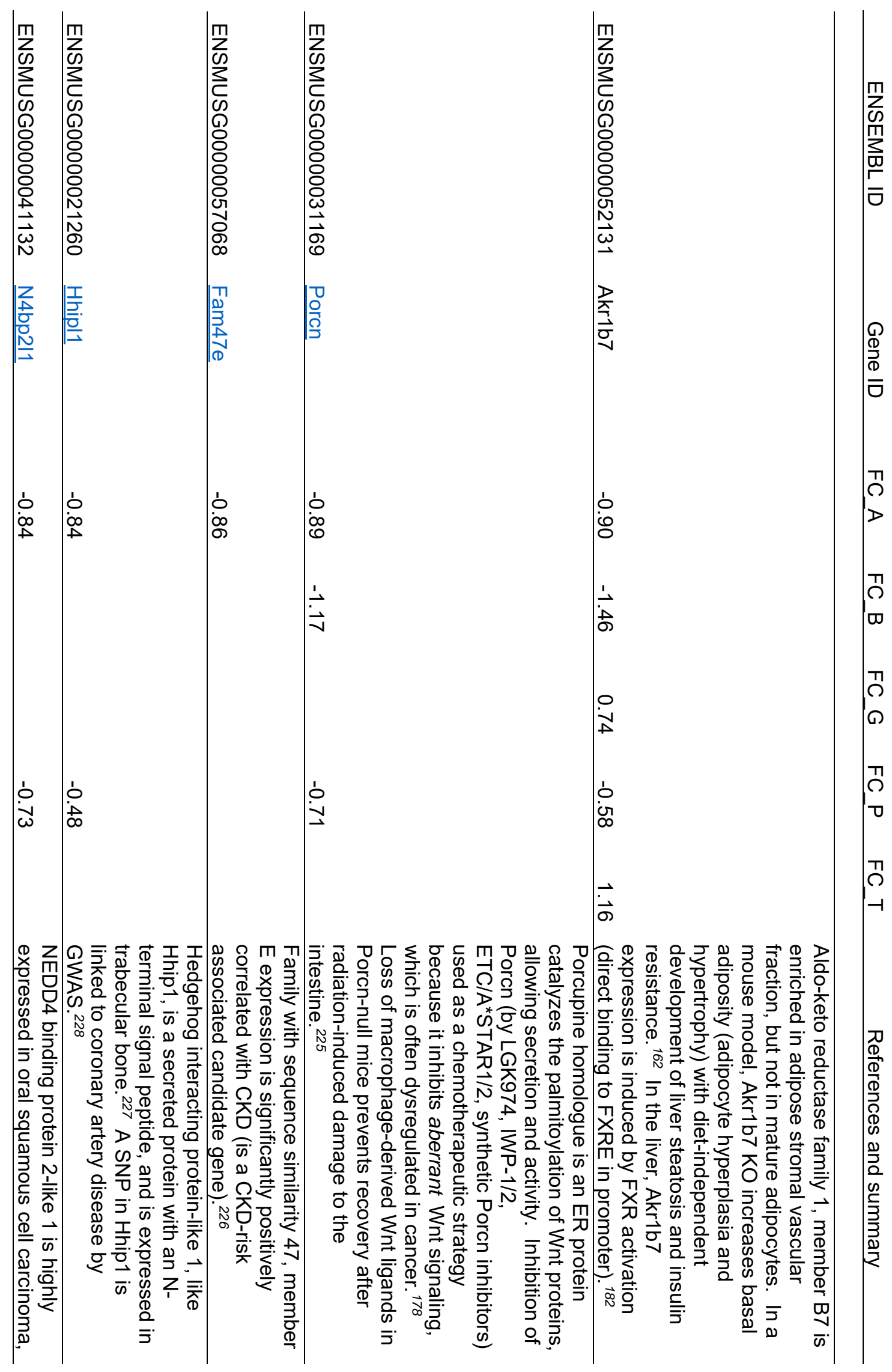




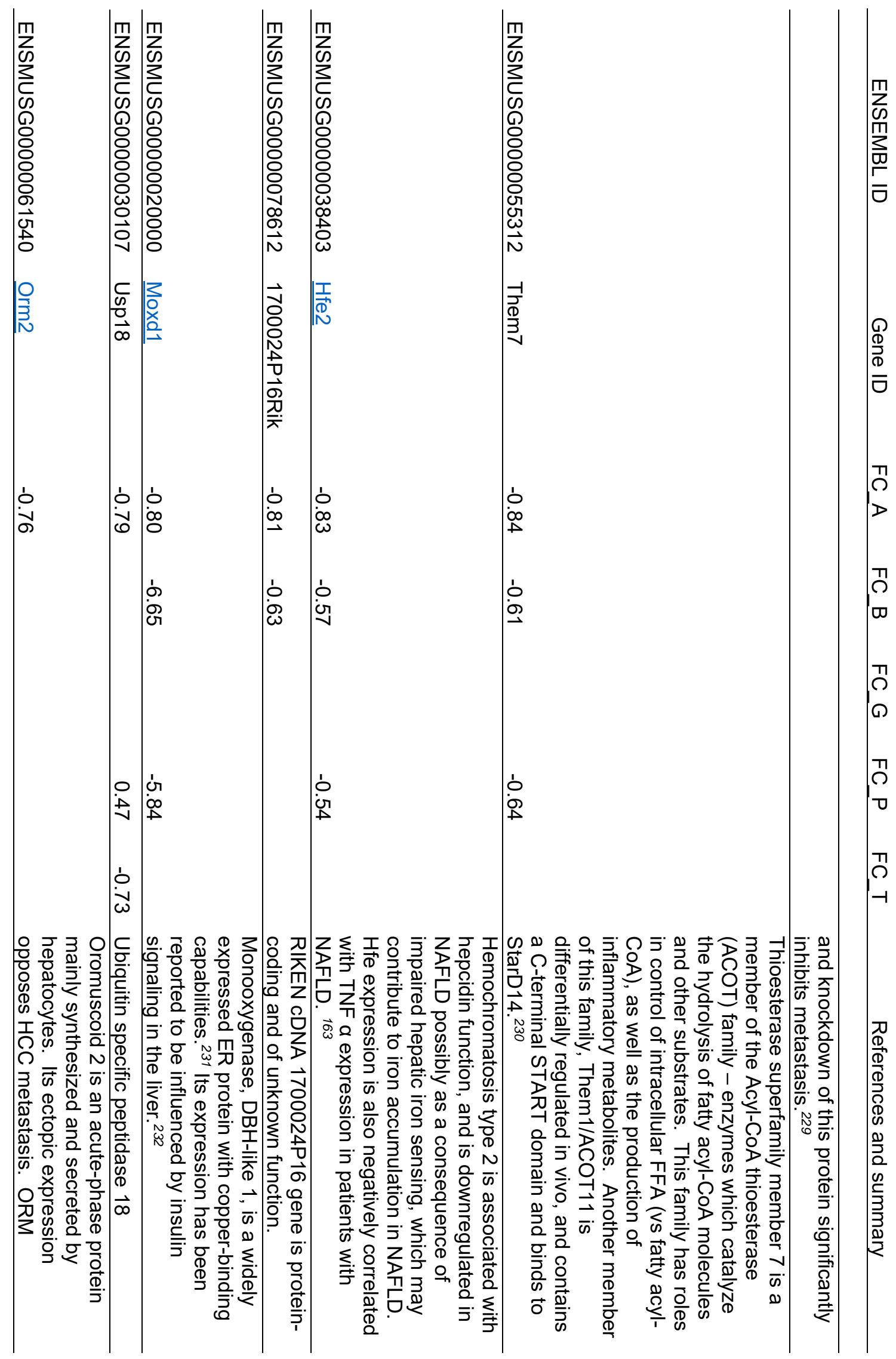




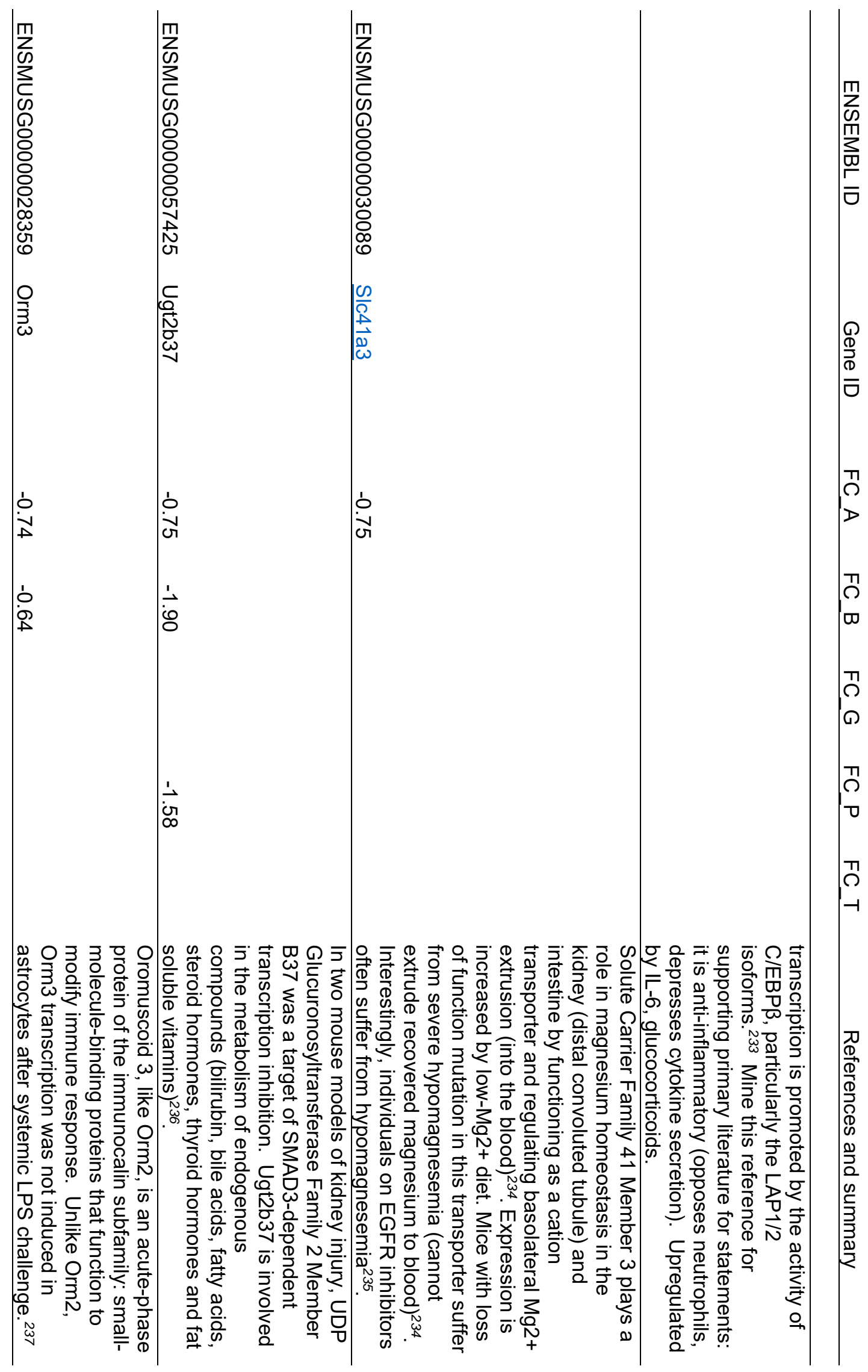




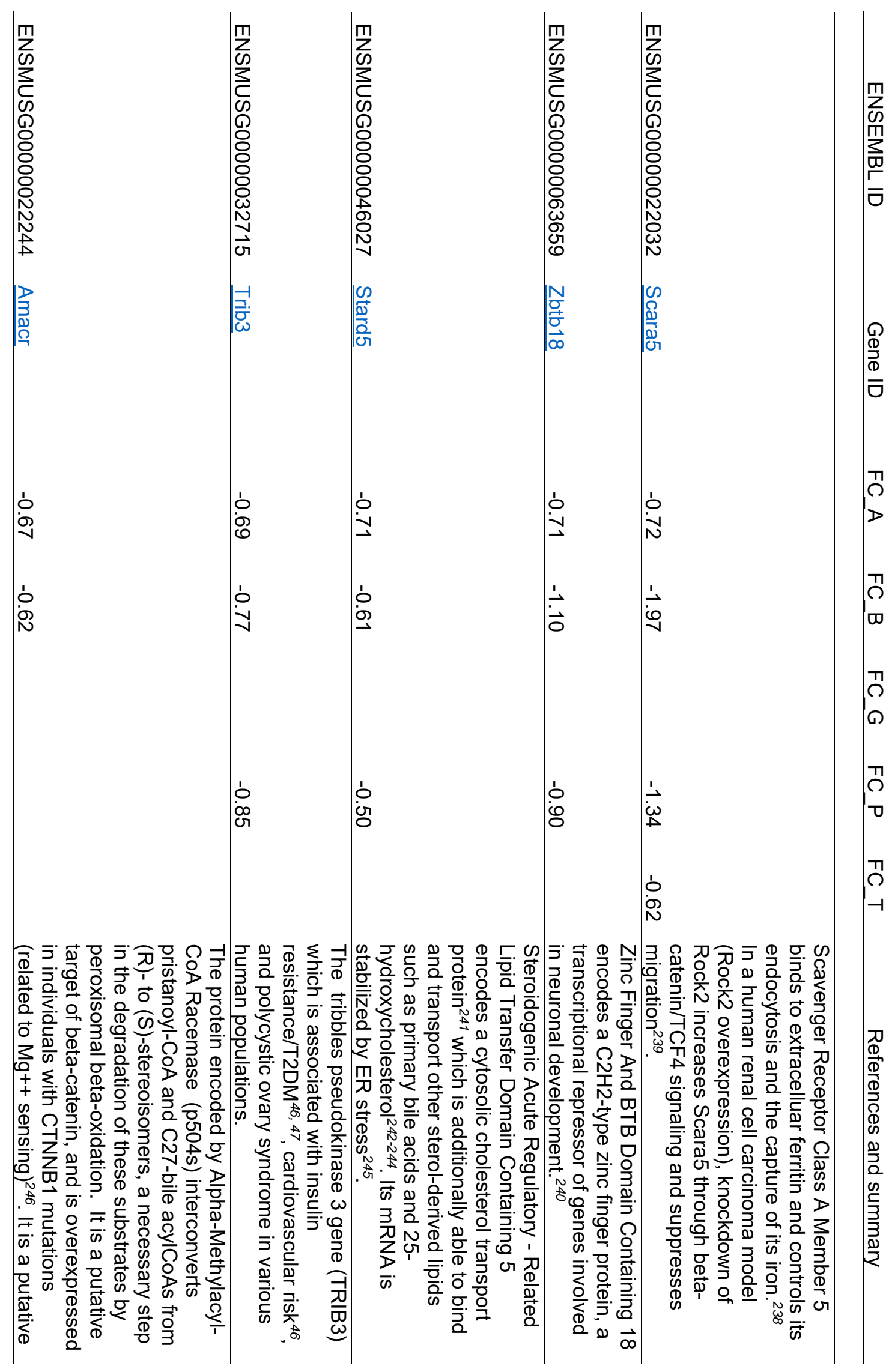




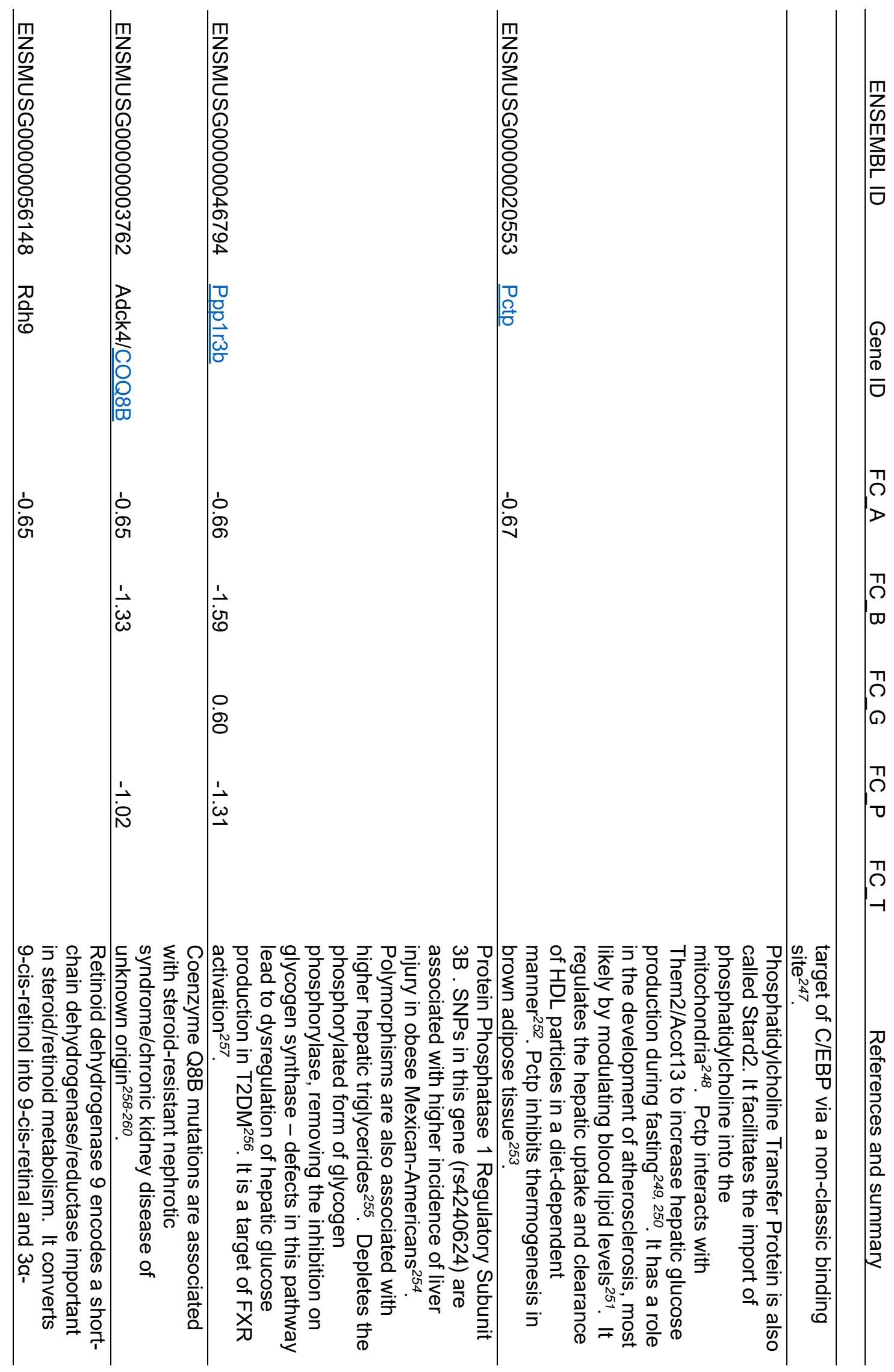




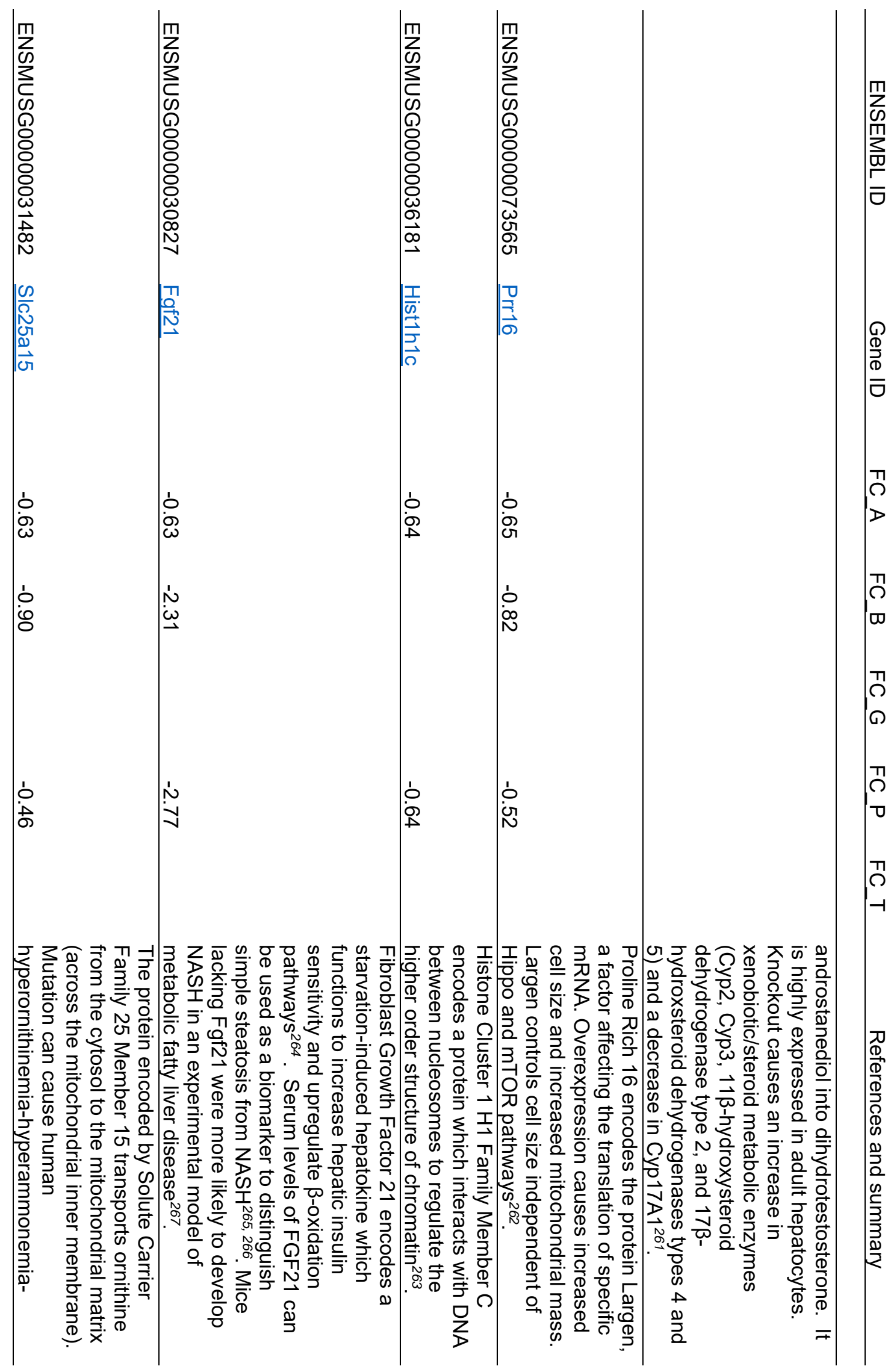




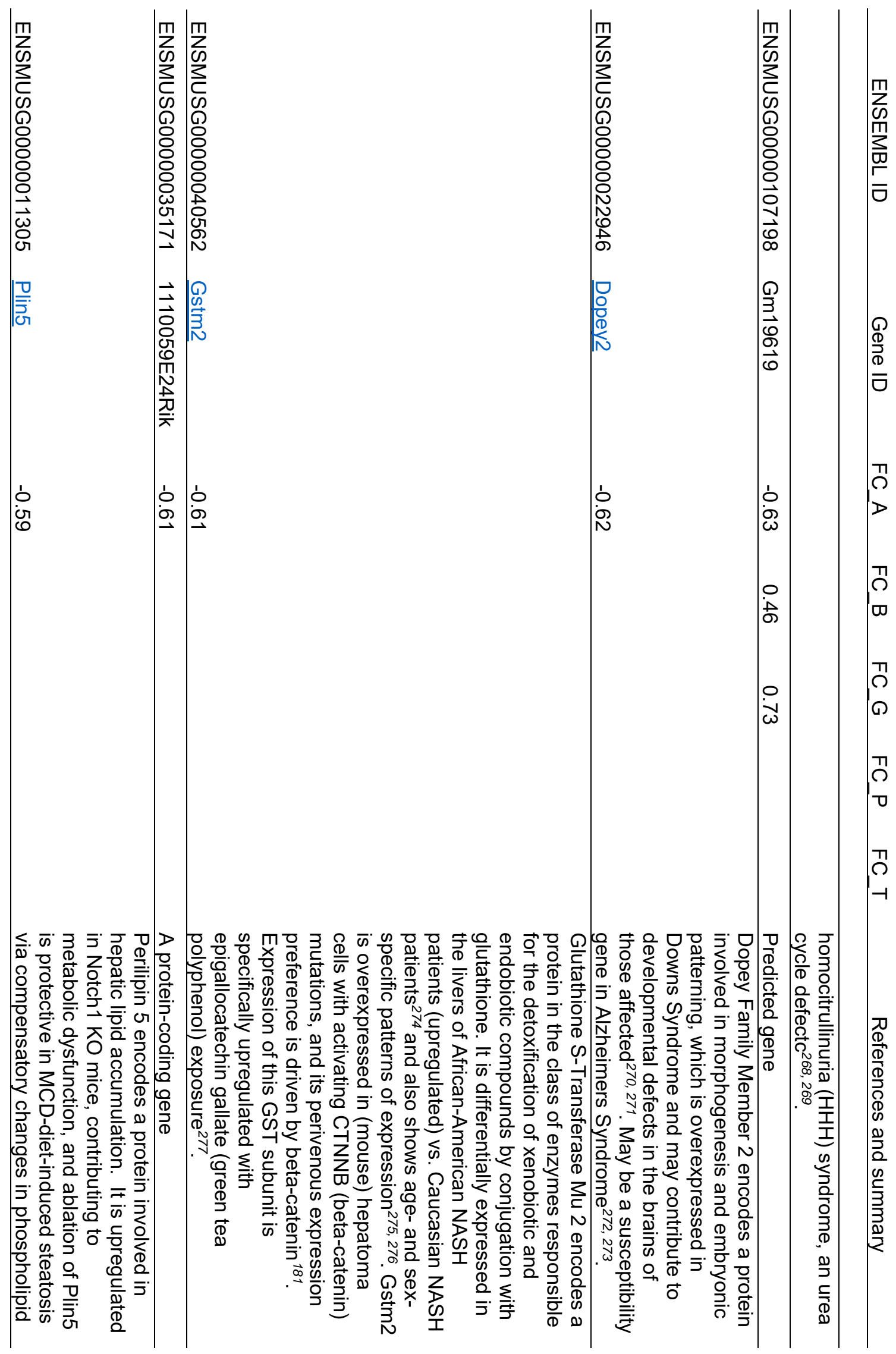




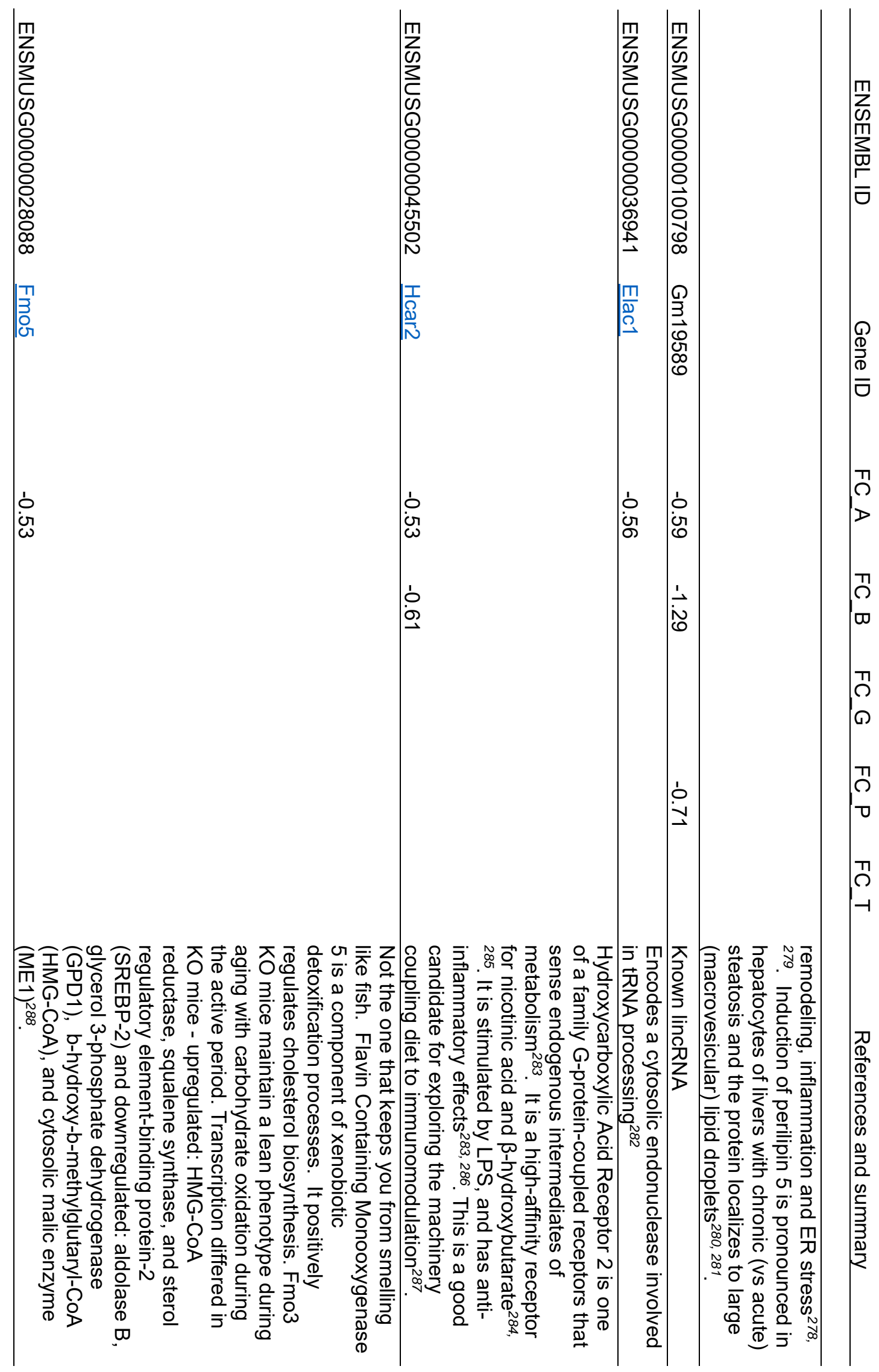




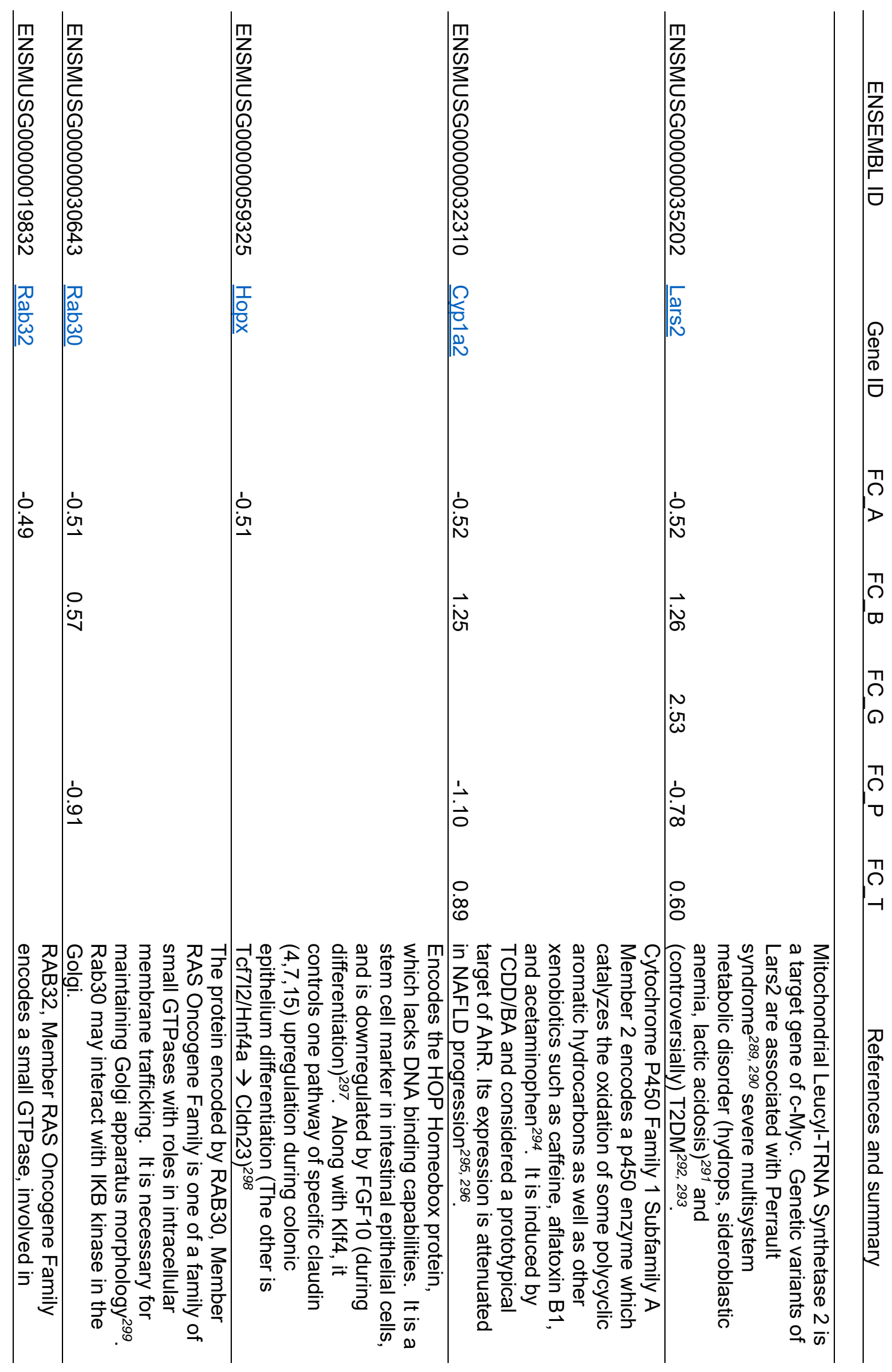




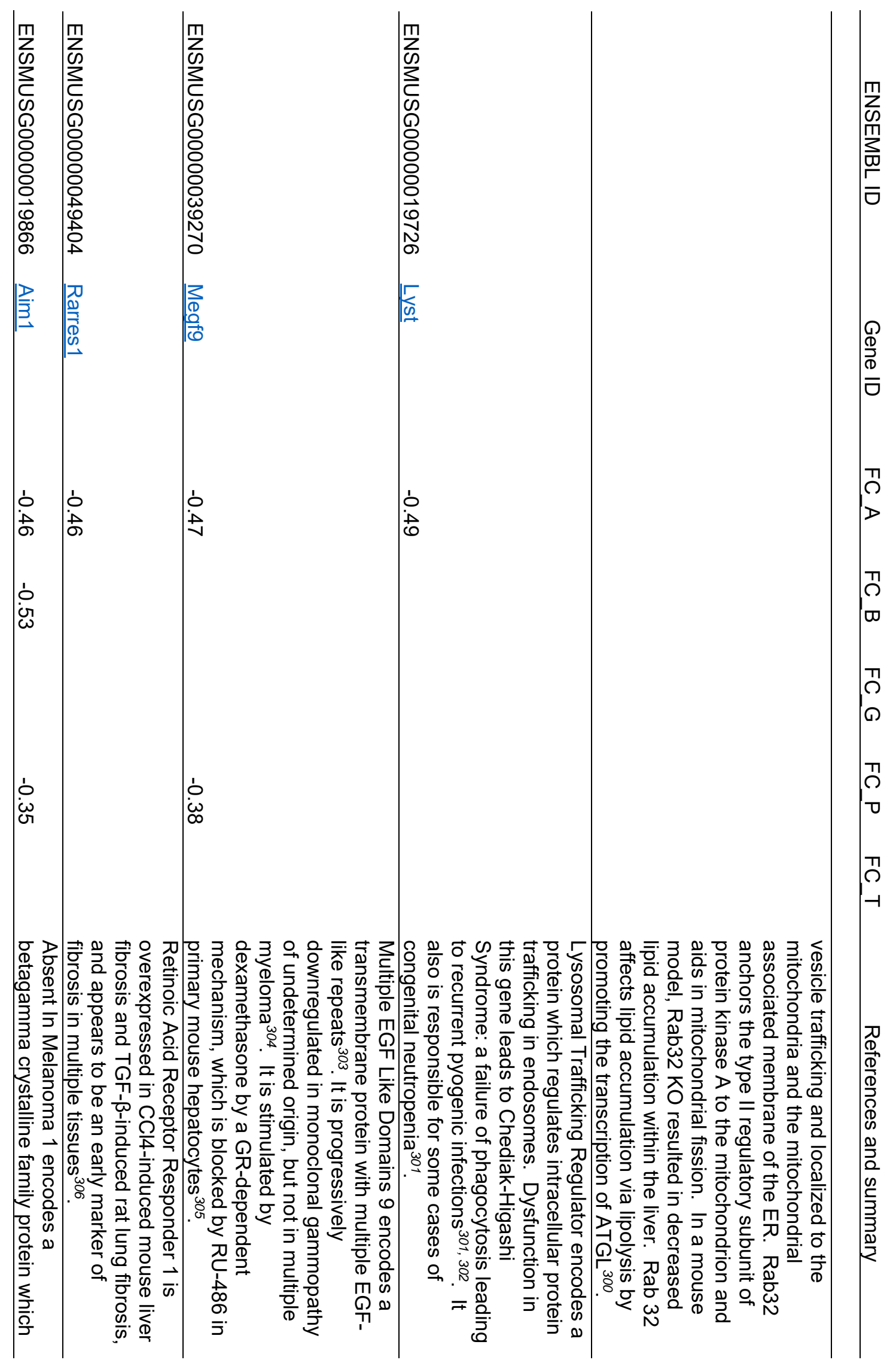



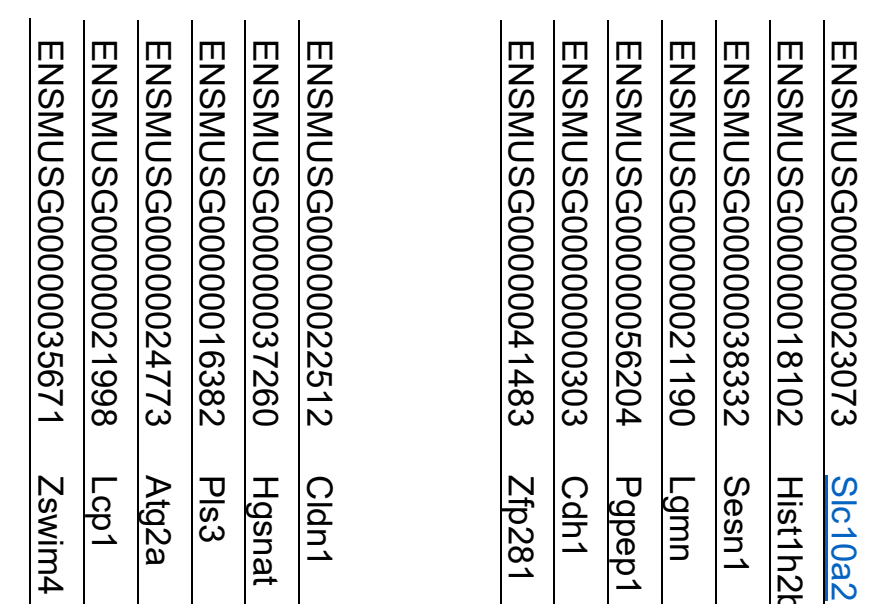

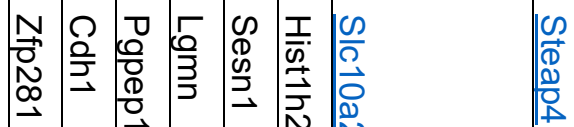

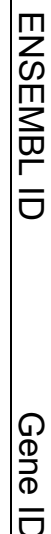

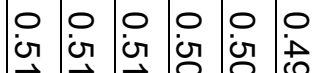

- 0 .

के मे है
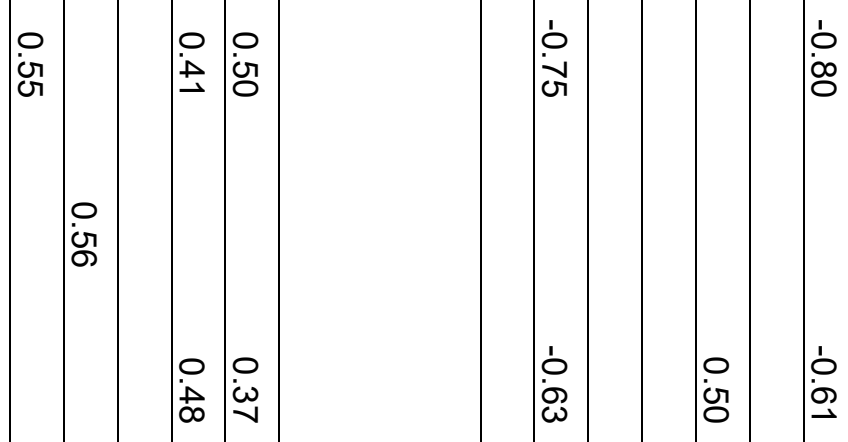

กூ

$\infty$

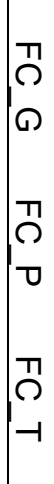

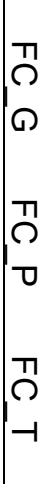

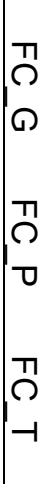

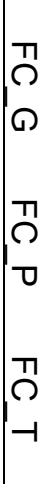

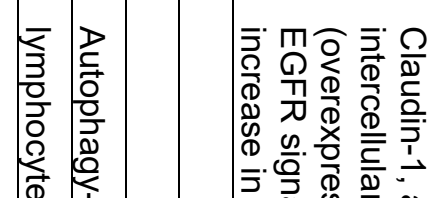

\section{竞}

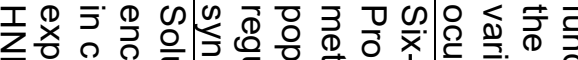

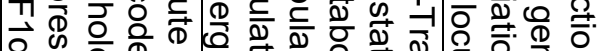
을 을

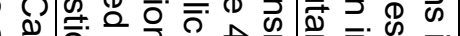

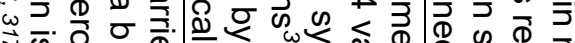

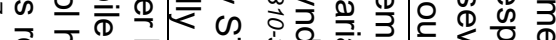

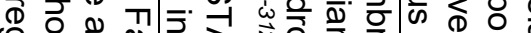

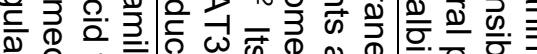
证 囚 б

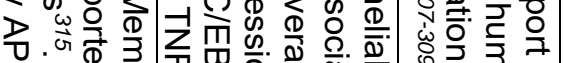

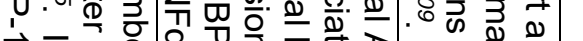

$\overrightarrow{0} \vec{\omega}$

Q

(1)

กึ

D $-1$ 


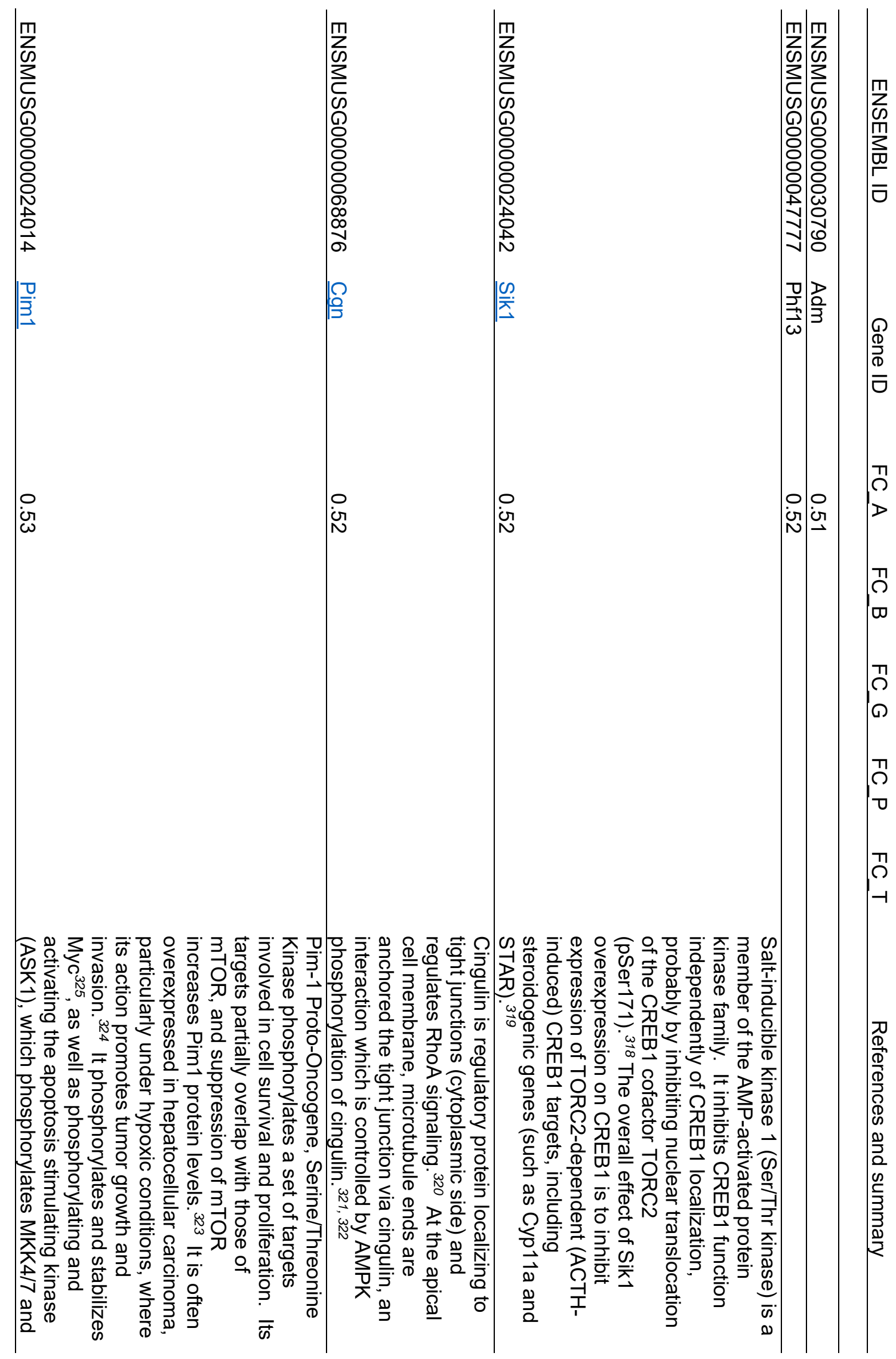




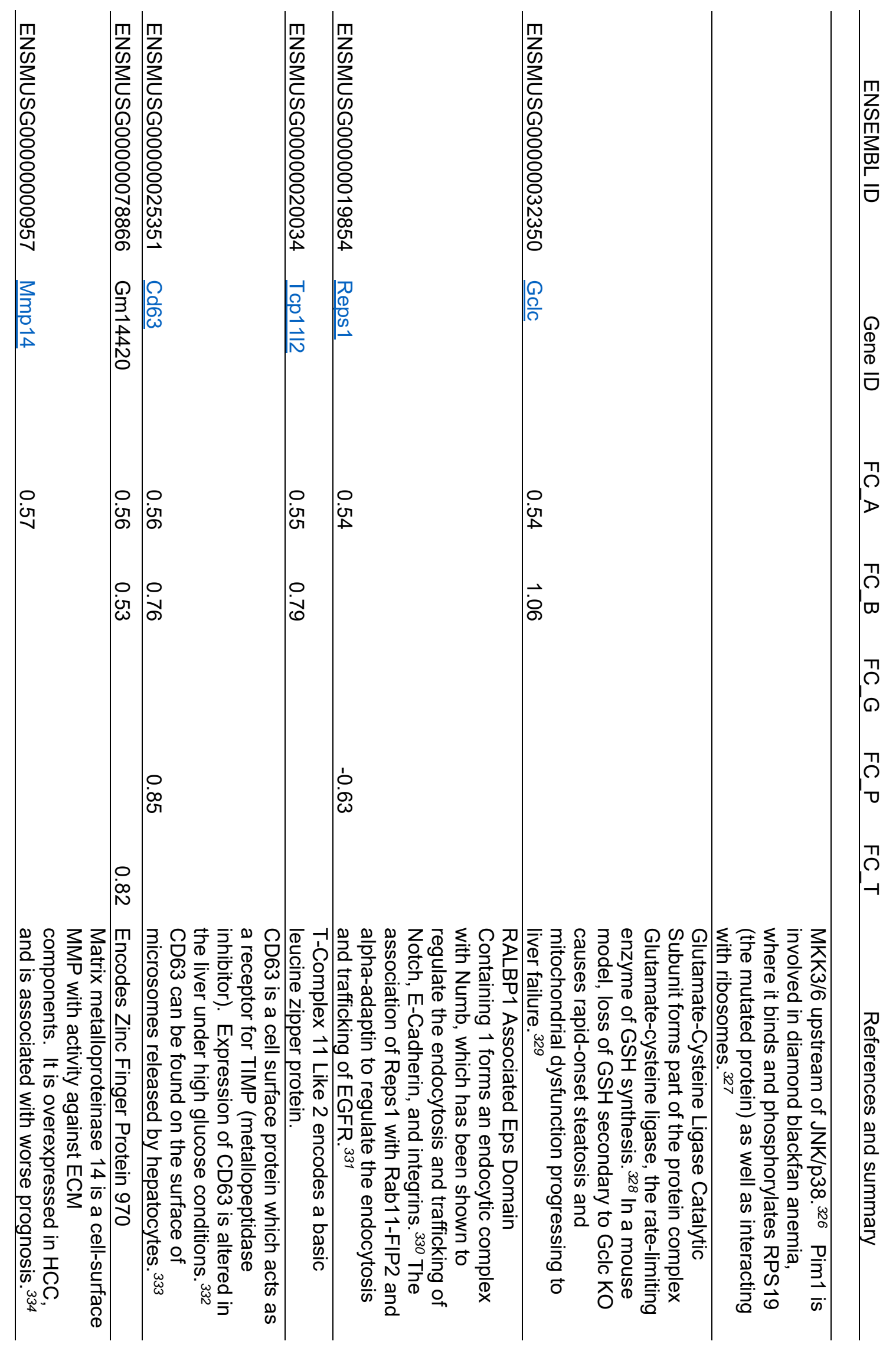




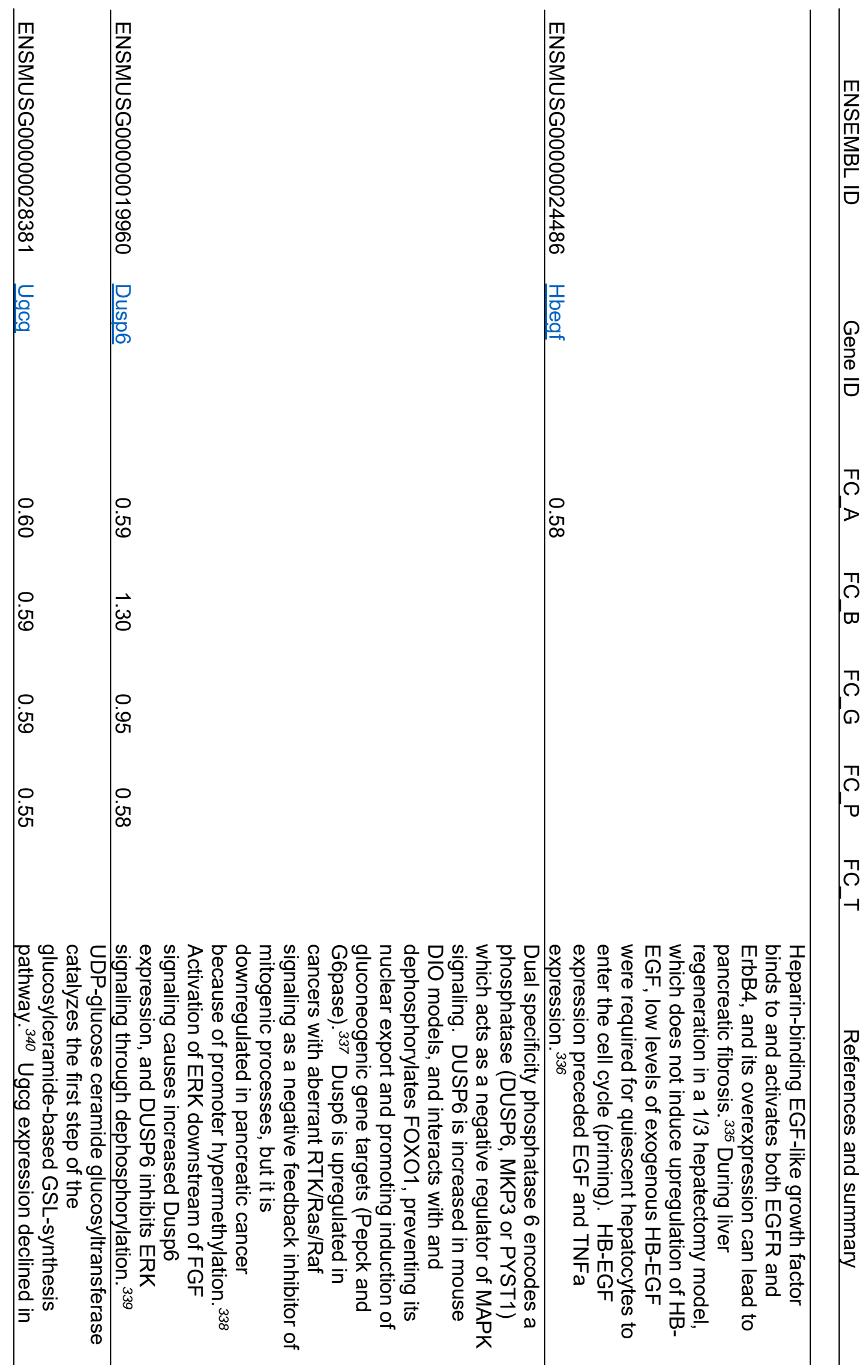




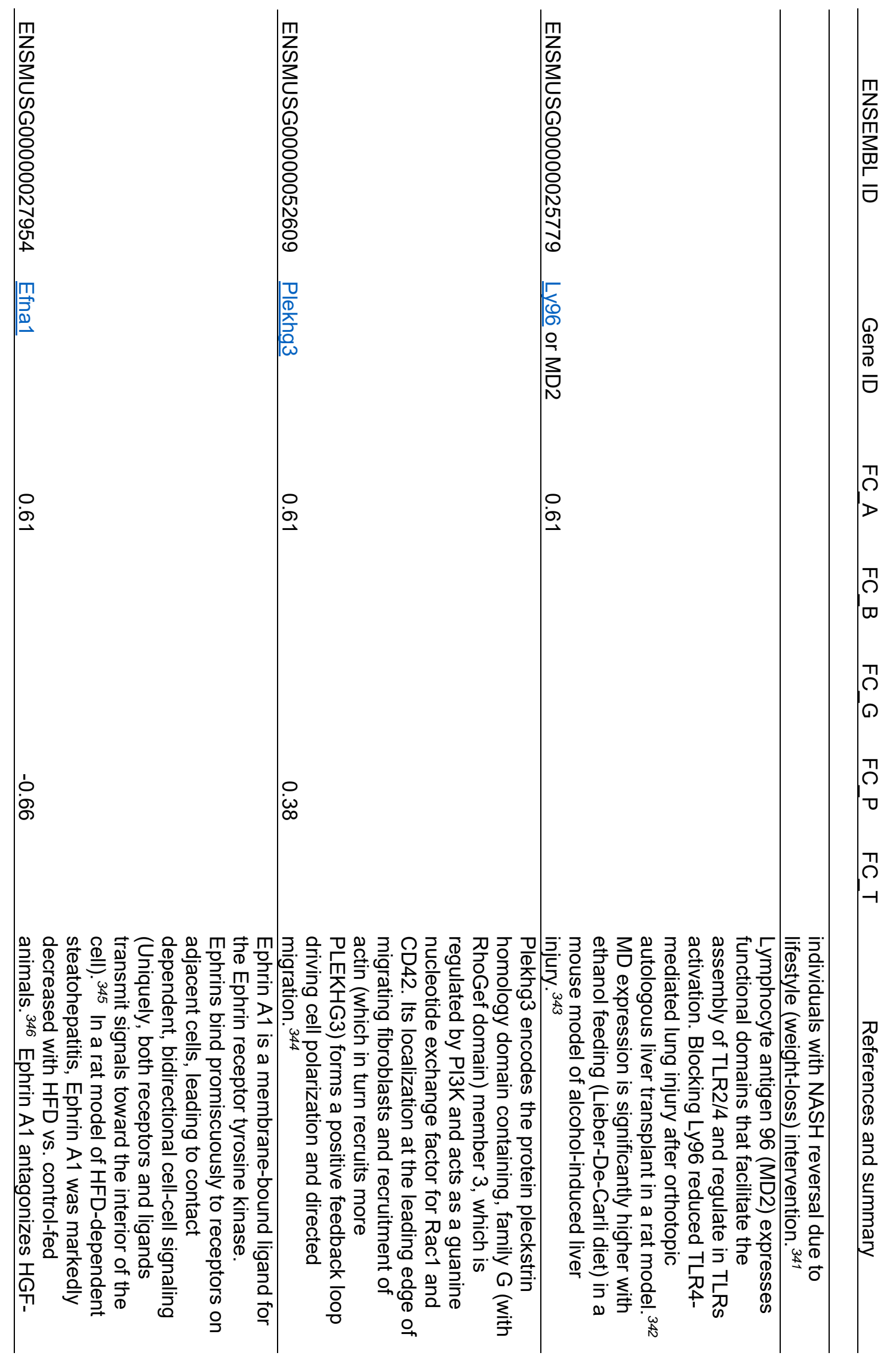




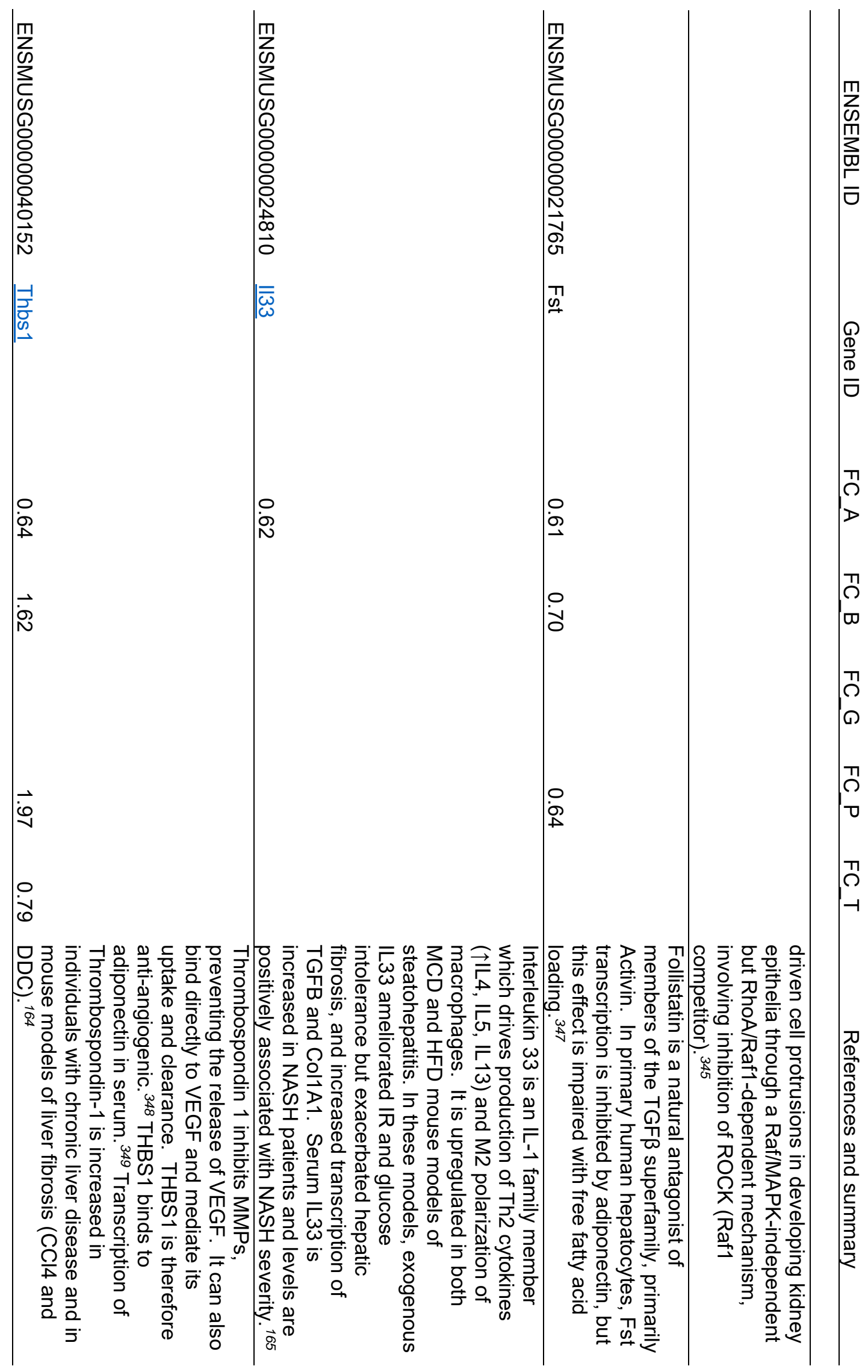




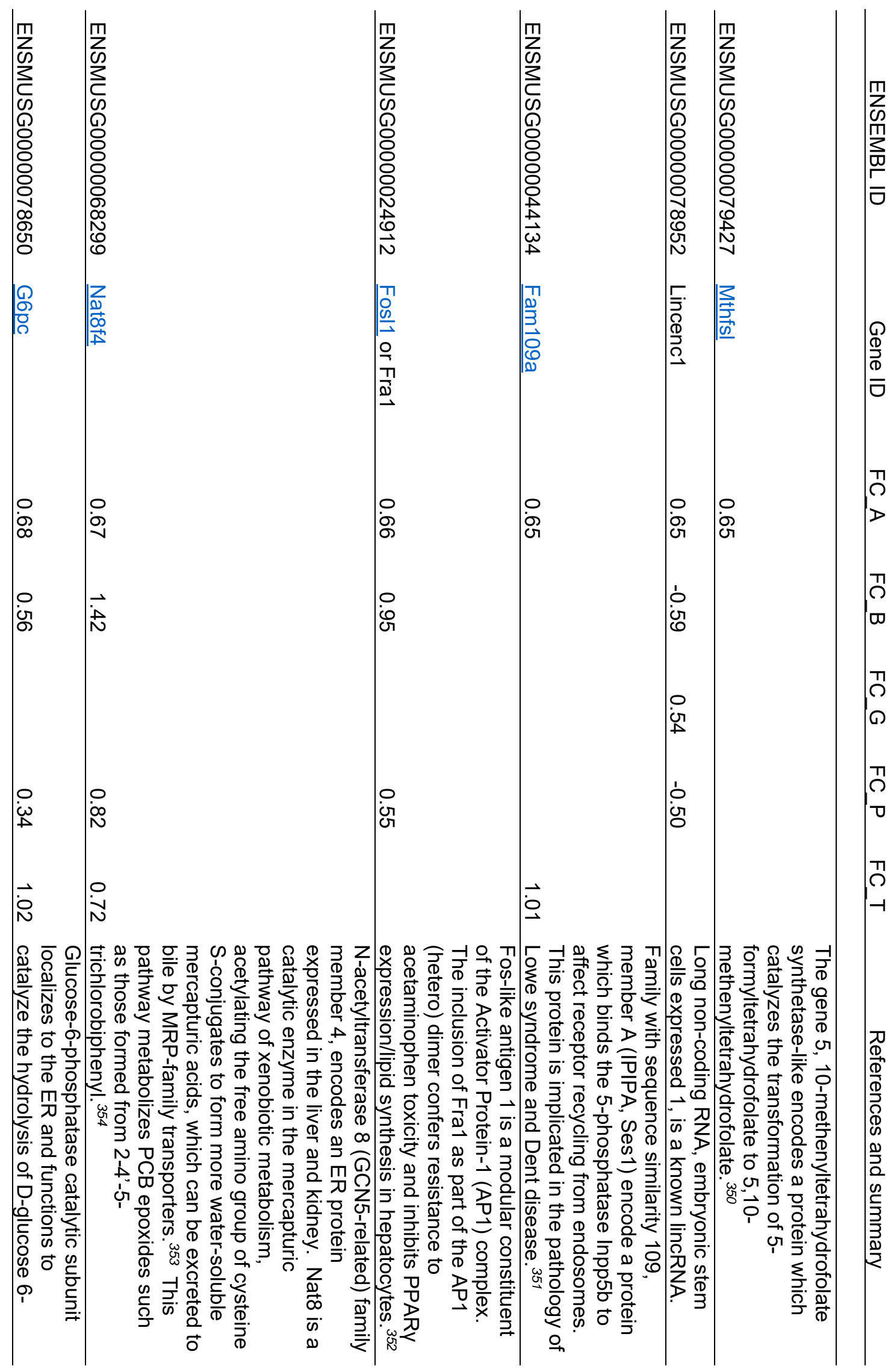




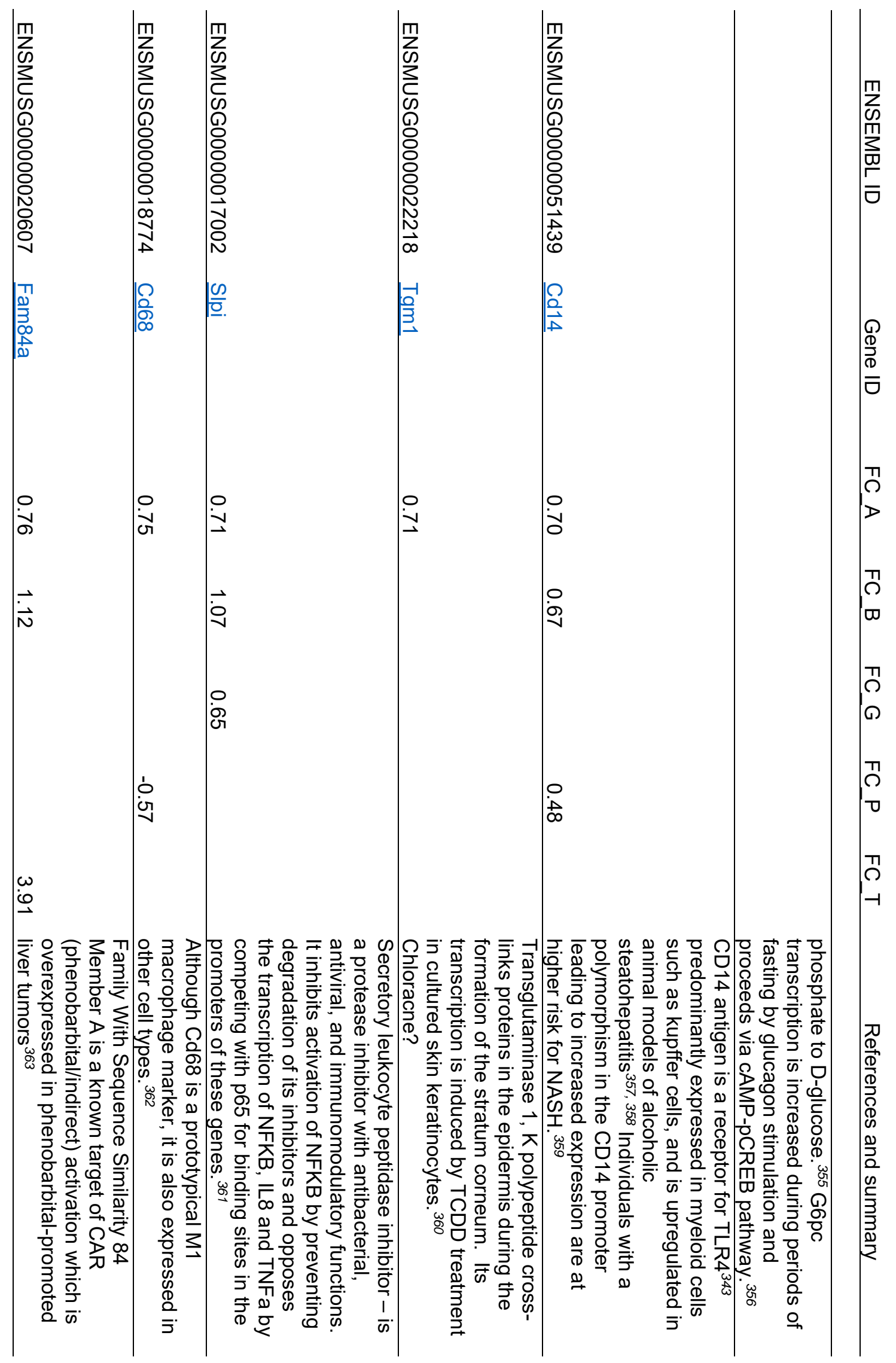




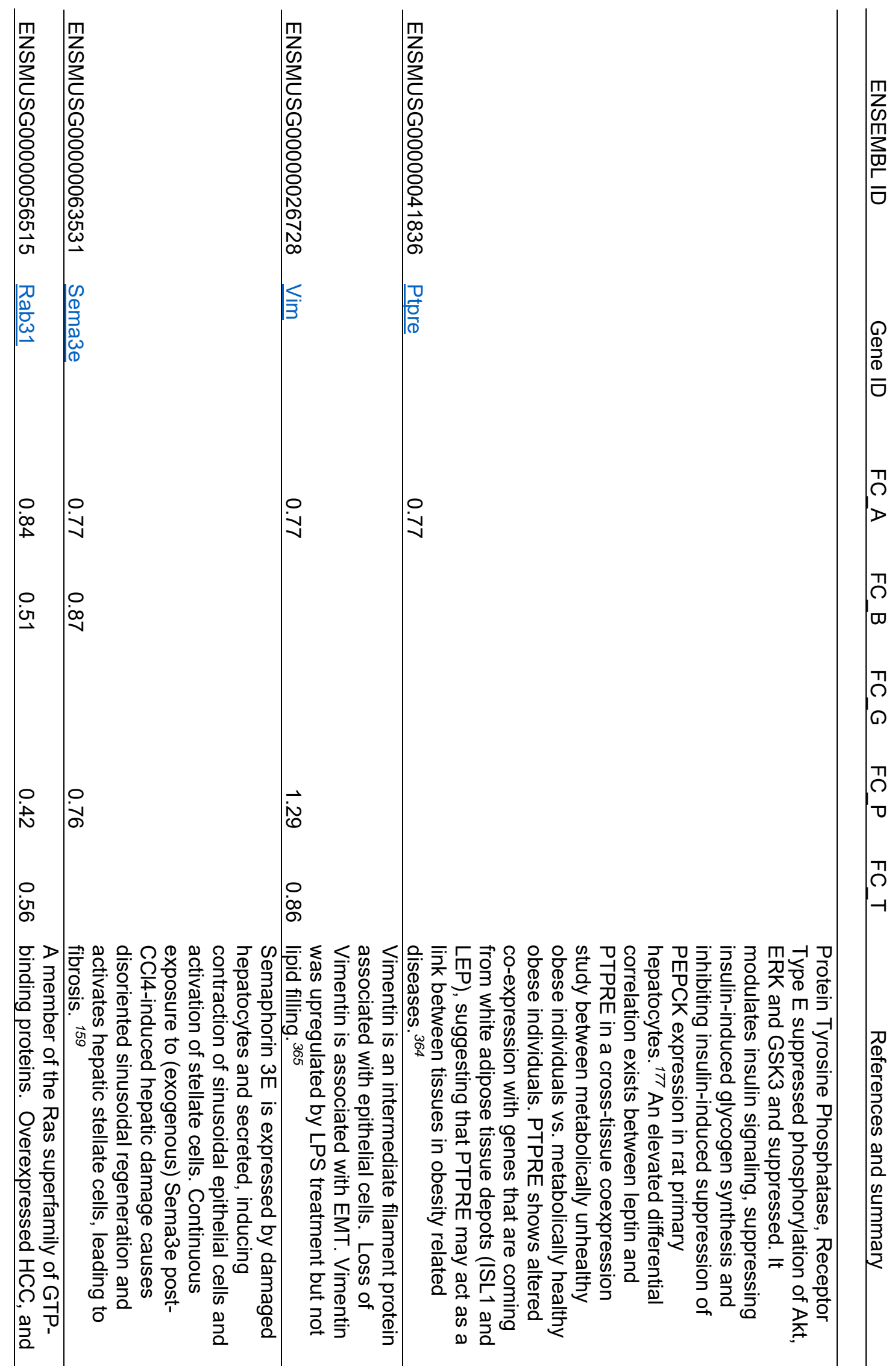




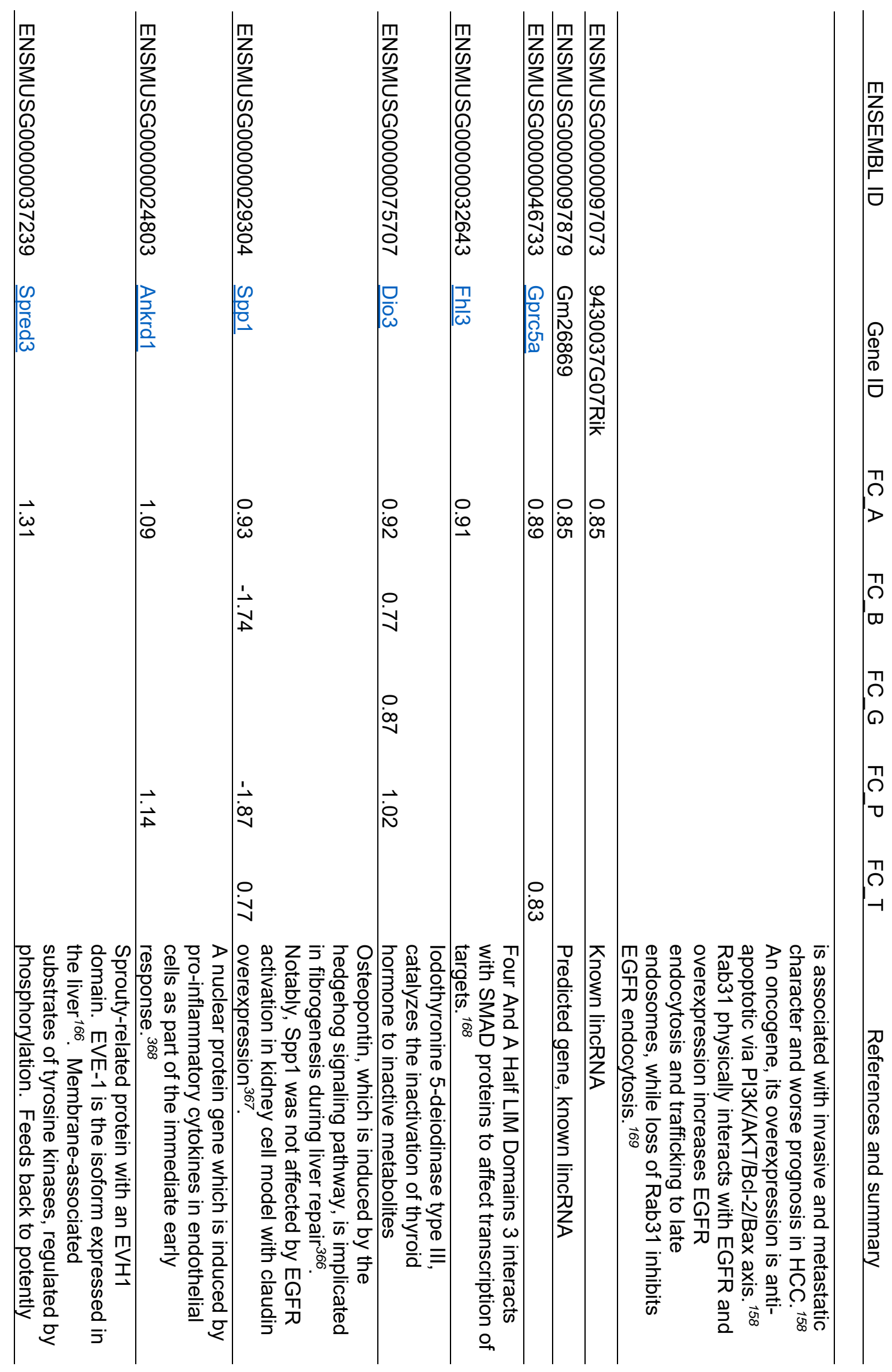




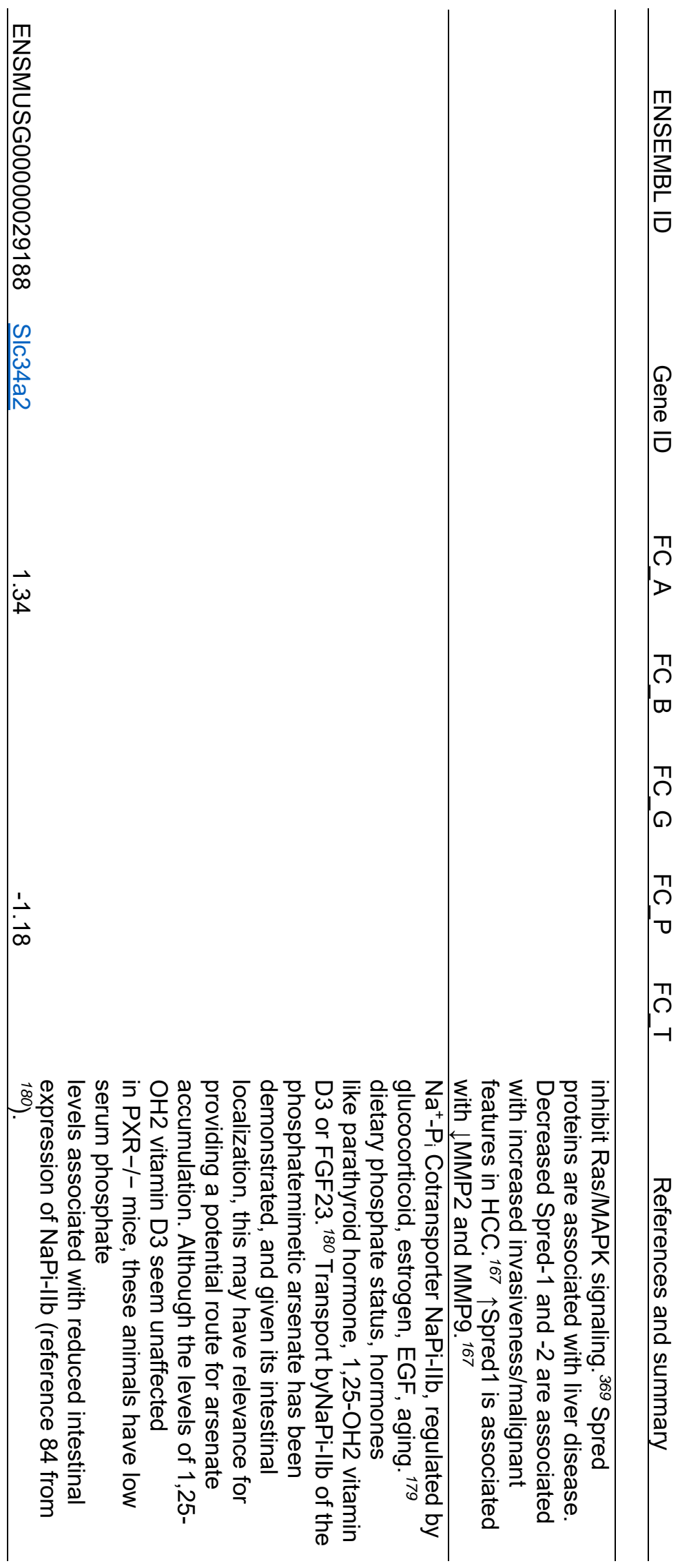




\title{
CURRICULUM VITAE
}

\author{
NAME: $\quad$ Heather Brooke Clair \\ DATE OF $\quad$ Clearwater, FL - April 12, 1977 \\ BIRTH: \\ EDUCATION Master of Science \\ AND TRAINING: University of Louisville Department of Biochemistry and Molecular Genetics \\ May, 2015 \\ Master of Science in Agriculture \\ University of Georgia College of Agricultural and Environmental Science \\ August, 2001 \\ Bachelor of Science - Animal Science \\ University of Georgia College of Agricultural and Environmental Science \\ May, 1999 \\ Bachelor of Science - Dairy Science \\ University of Georgia College of Agricultural and Environmental Science \\ May, 1999 \\ AWARDS: $\quad$ Travel award - NIEHS 25 Years of Endocrine Disruption Meeting \\ 2016 \\ Ruth L. Kirschstein National Research Service Award Individual Fellowship \\ (F30ES025099) \\ 2015-present \\ IPIBS Fellowship \\ 2013-2014 \\ Summer Research Scholar Program Fellowship (UofL School of Medicine) \\ 2011-2012 \\ Cum laude, BS in Animal Science, BS in Dairy Science (University of \\ Georgia) \\ May, 1999 \\ University of Georgia Honors Program \\ 1995-1999 \\ HOPE Scholarship (Awarded by State of Georgia) \\ 1995-1999 \\ Georgia Governor's Scholarship (Awarded by State of Georgia) \\ 1995-1999 \\ Governor's Honors Program (State of Georgia) \\ 1994
}


PROFESSIONAL American Physician Scientist Association

SOCIETIES: 2013-present

Society of Toxicology

2013-present

American Association for the Study of Liver Disease

2016

PUBliCATIONS: Clair, H. B., Pinkston, C., Pavuk, M., Dutton, N. D., Brock, G., Prough, R. A., Falkner, K. C., Wahlang, B., McClain, C., and Cave, M. C. (under review) High Prevalence of Human Environmental Liver Disease and Suspected Toxicant Associated Steatohepatitis in a Large United States Residential Cohort with High Polychlorinated Biphenyl Exposures

Wahlang, B., Prough, R. A., Falkner, K. C., Hardesty, J. E., Song, M., Clair, H. B., Clark, B. J., States, J. C., Arteel, G. E., and Cave, M. C. (2016) Polychlorinated Biphenyl-Xenobiotic Nuclear Receptor Interactions Regulate Energy Metabolism, Behavior, and Inflammation in Nonalcoholic-Steatohepatitis, Toxicol Sci 149, 396-410.

Al-Eryani, L., Wahlang, B., Falkner, K. C., Guardiola, J. J., Clair, H. B., Prough, R. A., and Cave, M. (2015) Identification of Environmental Chemicals Associated with the Development of Toxicant-associated Fatty Liver Disease in Rodents, Toxicol Pathol 43, 482-497.

Cave, M. C., Clair, H. B., Hardesty, J. E., Falkner, K. C., Feng, W., Clark, B. J., Sidey, J., Shi, H., Aqel, B. A., McClain, C. J., and Prough, R. A. (2016) Nuclear receptors and nonalcoholic fatty liver disease, Biochim Biophys Acta 1859, 1083-1099.

Hardesty, J. E., Wahlang, B., Falkner, K. C., Clair, H. B., Clark, B. J., Ceresa, B. P., Prough, R. A., and Cave, M. C. (2017) Polychlorinated biphenyls disrupt hepatic epidermal growth factor receptor signaling, Xenobiotica 47, 807-820.

Wahlang, B., Falkner, K. C., Clair, H. B., Al-Eryani, L., Prough, R. A., States, J. C., Coslo, D. M., Omiecinski, C. J., and Cave, M. C. (2014) Human receptor activation by aroclor 1260 , a polychlorinated biphenyl mixture, Toxicol Sci 140, 283-297.

Wahlang, B., Song, M., Beier, J. I., Cameron Falkner, K., Al-Eryani, L., Clair, H. B., Prough, R. A., Osborne, T. S., Malarkey, D. E., Christopher States, J., and Cave, M. C. (2014) Evaluation of Aroclor 1260 exposure in a mouse model of diet-induced obesity and non-alcoholic fatty liver disease, Toxicol Appl Pharmacol 279, 380-390.

Wahlang, B., Beier, J. I., Clair, H. B., Bellis-Jones, H. J., Falkner, K. C., McClain, C. J., and Cave, M. C. (2013) Toxicant-associated steatohepatitis, Toxicol Pathol 41, 343-360. 
Gharib, S. A., Dayyat, E. A., Khalyfa, A., Kim, J., Clair, H. B., Kucia, M., and Gozal, D. (2010) Intermittent hypoxia mobilizes bone marrow-derived very small embryonic-like stem cells and activates developmental transcriptional programs in mice, Sleep 33, 1439-1446.

Gharib, S. A., Khalyfa, A., Kucia, M. J., Dayyat, E. A., Kim, J., Clair, H. B., and Gozal, D. (2011) Transcriptional landscape of bone marrow-derived very small embryonic-like stem cells during hypoxia, Respir Res 12, 63.

Kheirandish-Gozal, L., Bhattacharjee, R., Kim, J., Clair, H. B., and Gozal, D. (2010) Endothelial progenitor cells and vascular dysfunction in children with obstructive sleep apnea, Am J Respir Crit Care Med 182, 92-97.

Kim, J., Bhattacharjee, R., Dayyat, E., Snow, A. B., Kheirandish-Gozal, L., Goldman, J. L., Li, R. C., Serpero, L. D., Clair, H. B., and Gozal, D. (2009) Increased cellular proliferation and inflammatory cytokines in tonsils derived from children with obstructive sleep apnea, Pediatr Res 66, 423428.

Li, R. C., Lee, S. K., Pouranfar, F., Brittian, K. R., Clair, H. B., Row, B. W., Wang, Y., and Gozal, D. (2006) Hypoxia differentially regulates the expression of neuroglobin and cytoglobin in rat brain, Brain Res 1096, 173-179.

Arnold, H. B., Della-Fera, M. A., and Baile, C. A. (2001) Review of myostatin history, physiology and applications., LifeXY 1, 1014-1022.

Lin, J., Arnold, H. B., Della-Fera, M. A., Azain, M. J., Hartzell, D. L., and Baile, C. A. (2002) Myostatin knockout in mice increases myogenesis and decreases adipogenesis, Biochem Biophys Res Commun 291, 701-706.

ABSTRACTS

AND CONFERENCES:
Clair, H., Falkner, K. C., Hardesty, J., Kalbfleisch, T., Prough, R., and Cave, M. C. (2017) Murine primary hepatocytes exposed to AHR, PXR, CAR or LXR agonists produce differential transcriptomes which partially overlap with one another and the transcriptome induced by PCB exposure. Gastroenterology 152, S1158-S1158. (Poster: Digestive Disease Week. May 06-09, 2017. Chicago, IL)

Hardesty, J., Clair, H., Falkner, C., Shi, H., Wahlang, B., Wilkey, D., Prough, R., Merchant, M., and Cave, M. C. (2017). Hepatic proteome alterations due to PCB exposure and diet in the development of NASH, Gastroenterology 152, S1114-S1114. (Poster: Digestive Disease Week. May 06-09, 2017. Chicago, IL)

Shi, H., Clair, H., Hardesty, J., Jin, J., Falkner, C., Prough, R., Song, M., and Cave, M. C. (2017) The role of arylhydrocarbon receptor in PCBinduced fatty liver disease in mice, Gastroenterology 152, S1108-S1109. (Poster: Digestive Disease Week. May 06-09, 2017. Chicago, IL)

Vatsalya, V., Clair, H., Falkner, K. C., Cave, M. C., and McClain, C. J. (2017). Efficacy of Keratin 18 (CK18) as a biomarker of severity of liver injury in acute alcoholic hepatitis, Gastroenterology 152, S1110-S1111. (Poster: Digestive Disease Week. May 06-09, 2017. Chicago, IL) 
Clair, H.B., Hardesty, J.E., Falkner, K.C., Kalbfleisch, T., Prough, R.A., Cave, M.C. Cyclic AMP-Responsive Element Binding protein (CREB-1) Is a Common Regulator of Genes Differentially Transcribed with Aroclor 1260 Exposure. The Toxicologist: Supplement to Toxicological Sciences, 156(1). Society of Toxicology, 2017. Abstract no. 1371. (Poster: 56 ${ }^{\text {th }}$ Annual Meeting of the Society of Toxicology. March 12-16, 2017. Baltimore, MD.)

Falkner, K.C., Clair, H.B., Hardesty, J.E., Kalbfleisch, T., Prough, R.A., Cave, M.C. In Murine Primary Hepatocytes, Genes Differentially Transcribed with PCB Treatment Partially Overlap with AhR, PXR, CAR, and LXR-Dependent Differential Transcriptomes. The Toxicologist: Supplement to Toxicological Sciences, 156(1). Society of Toxicology, 2017. Abstract no. 1118. (Poster: 56 $6^{\text {th }}$ Annual Meeting of the Society of Toxicology. March 12-16, 2017. Baltimore, MD.)

Cave, M.C., Clair, H.B., Pinkston, C.M., Falkner, K.C., Birnbaum, L.S., Pavuk, M. Initial Analysis of Liver Disease in the Anniston Community Health Survey-II. M.C. Cave1, H.B. The Toxicologist: Supplement to Toxicological Sciences, 156(1). Society of Toxicology, 2017. Abstract no. 1298. (Poster: $56^{\text {th }}$ Annual Meeting of the Society of Toxicology. March $12-$ 16, 2017. Baltimore, MD.)

Pinkston, C.M., Middleton, F.A., Rosenbaum, P.F., Clair, H.B., Falkner, K.C., Pavuk, M., Birnbaum, L.S., Cave, M.C. Associations between PCB and Dioxin Exposures with Serum Cytokines in the Anniston Community Health Survey-II. The Toxicologist: Supplement to Toxicological Sciences, 156(1). Society of Toxicology, 2017. Abstract no. 1299. (Poster: $56^{\text {th }}$ Annual Meeting of the Society of Toxicology. March 12-16, 2017. Baltimore, MD.)

Hardesty, J., Falkner, C., Clair, H, Shi, H., Wahlang, B., Wilkey, D., Prough, R., Merchant, M., Cave, M., Hepatic Proteome Alterations Due to PCB Exposure and a High-Fat Diet. The Toxicologist: Supplement to Toxicological Sciences, 156(1). Society of Toxicology, 2017. Abstract no.1114. (Poster: $56^{\text {th }}$ Annual Meeting of the Society of Toxicology. March 12-16, 2017. Baltimore, MD.)

Schecter, A.J., Kincaid, J.S., Clair, H., Cave, M., Bhatnagar, A., Riggs, D., Birnbaum, L. Hepatic, Cardiovascular, and Other Biomarkers Associated with Organics and Metals Exposure in Female Vietnamese Electronic Waste Workers and Comparisons. The Toxicologist: Supplement to Toxicological Sciences, 156(1). Society of Toxicology, 2017. Abstract no. 1905. (Poster: $56^{\text {th }}$ Annual Meeting of the Society of Toxicology. March 1216, 2017. Baltimore, MD.)

Chorley, B., Carswell, G., Nelson, G.M., Angrish, M.M., Clair, H., Pinkston, C.M., Pavuk, M. Serum microRNA Biomarker Identification in a Residential Cohort with Elevated Polychlorinated Biphenyl Exposures. The Toxicologist: Supplement to Toxicological Sciences, 156(1). Society of Toxicology, 2017. Abstract no. 2479. (Poster: $56^{\text {th }}$ Annual Meeting of the Society of Toxicology. March 12-16, 2017. Baltimore, MD.) 
Shi, H., Clair, H.B., Hardesty, J.E., Jin, J., Falkner, K., Prough, R.A., Song, M., Cave, M.C. The Role of Aryl Hydrocarbon Receptor in PCB-Induced Fatty Liver Disease in Mice. The Toxicologist: Supplement to Toxicological Sciences, 156(1). Society of Toxicology, 2017. Abstract no. 2523. (Poster: $56^{\text {th }}$ Annual Meeting of the Society of Toxicology. March 12-16, 2017. Baltimore, MD.)

Vatsalya, V., Clair, H., Falkner, K. C., Cave, M. C., and McClain, C. J. (2017) Efficacy of CK18 as a biomarker of severity of liver injury in acute alcoholic hepatitis. Alcoholism-Clinical and Experimental Research 41, 226A-226A. (Poster: 40th Annual Scientific Meeting of the ResearchSociety-on-Alcoholism. Jun 24-28, 2017. Denver, CO)

Hardesty, J., Clair, H. B., Shi, H., Falkner, C., Prough, R., Wilkey, D., Merchant, M. L., and Cave, M. (2016) Aroclor 1260 disrupts the hepatic proteome in the development of NASH, Hepatology 64, 787A-788A. (Poster: 67th Annual Meeting of the American-Association-for-the-Study-ofLiver-Diseases. Nov 11-15, 2016. Boston, MA)

Vatsalya, V., Cave, M., Clair, H. B., Burke, T., Falkner, K. C., and McClain, C. (2016) Role of CK18-M65 as a biomarker of acute alcoholic hepatitis and development of a modified MELD score using M65 and clinically relevant markers of severe AAH, Hepatology 64, 551A-551A. (Poster: 67th Annual Meeting of the American-Association-for-the-Study-of-Liver-Diseases. Nov 11-15, 2016. Boston, MA)

Clair, H.B., Pinkston, C.M., Pavuk, M., Brock, G., Prough, R.A., Falkner, K.C., McClain, C.J., and Cave, MC. PCB exposure is associated with a unique form of toxicant-associated steatohepatitis characterized by $\beta$-cell dysfunction and preserved insulin sensitivity. (Poster: 25 Years of Endocrine Disruption - NIEHS Conference. Bethesda, MD, 2016.)

Clair, H.B., Pinkston, C.M., Pavuk, M., Brock, G., Prough, R.A., Falkner, K.C., McClain, C.J., and Cave, MC. Follow-up evaluation of toxicant associated steatohepatitis in a PCB-exposed cohort reveals worsening liver injury despite reductions in serum PCB levels. (Poster: APSA/SMSS Regional joint meeting. Birmingham, AL, 2016.)

Falkner, K.C., Birnbaum, L., Schecter, A., Clair, H., and Cave, M.C. Adipocytokine and Liver Injury Biomarker Assessment in Electronic Waste Recyclers. The Toxicologist Supplement to Toxicological Sciences. 2016;

(1):2733A. Abstract no. 2733. (Poster: $55^{\text {th }}$ Annual Meeting of the Society of Toxicology. March 13-17, 2016. New Orleans, LA .)

Clair, H.B., Pinkston, C., Brock, G., Pavuk, M., Dutton, N., Falkner, K.C., Prough, R.A., Cave, M.C. Steatohepatitis Biomarkers Reveal Elevated Liver Disease in Polychlorinated Biphenyl-Exposed Cohort. The Toxicologist Supplement to Toxicological Sciences. 2016; (1):3170A. Abstract no. 3170. (Poster: $55^{\text {th }}$ Annual Meeting of the Society of Toxicology. March 13-17, 2016. New Orleans, LA .) 
Hardesty, J.E., Falkner, K.C., Prough, R.A., Clair, H.B., Wahlang, B., Cave, M.C. PCBs Diminish EGFR Signaling Pathways in OrganopollutantMediated Steatohepatitis. The Toxicologist Supplement to Toxicological Sciences. 2016; (1):1962A. Abstract no. 1962. (Poster: 55 ${ }^{\text {th }}$ Annual Meeting of the Society of Toxicology. March 13-17, 2016. New Orleans, LA .)

Clair, H., Pinkston, C., Dutton, N., Pavuk, M., Falkner, K.C., Wahland, B., Prough, R.A., Cave, M. Steatohepatitis Associated with Adipocytokine Abnormalities in the Anniston Community Health Survey. The Toxicologist CD Supplement to Toxicological Sciences - An Official Journal of the Society of Toxicology. 2015; 144(1). Abstract no. 769. (Poster: $54^{\text {th }}$ Annual Meeting of the Society of Toxicology. March 22-26, 2015. San Diego, CA)

Pavuk, M, Dutton, N., Clair, H. Wahlang, B., Pinkston, C., Cave, M. Polychlorinated Biphenyls, Diabetic Status, and Inflammatory Cytokines. The Toxicologist CD Supplement to Toxicological Sciences - An Official Journal of the Society of Toxicology. 2015; 144(1) Abstract no. 771. (Poster: $54^{\text {th }}$ Annual Meeting of the Society of Toxicology. March 22-26, 2015. San Diego, CA)

Wahlang, B., Falkner, K.C., Song, M., Clair, H., Prough, R.A., Cave, M., Role of Nuclear Receptors in Steatohepatitis Caused by Aroclor 1260 and High Fat Diet Coexposure. The Toxicologist CD Supplement to Toxicological Sciences - An Official Journal of the Society of Toxicology. 2015; 144(1). Abstract no. 2318. (Poster: 54 ${ }^{\text {th }}$ Annual Meeting of the Society of Toxicology. March 22-26, 2015. San Diego, CA)

Sidey, J., Clair, H., Hardesty, J., Falkner, K.C., Prough, R.A., Cave, M.C. Development Of A Primary Hepatocyte Model For TCE And PCE Toxicity. (Poster: Research! Louisville. 2015.)

Hardesty, J., Falkner, K.C., Clair, H., Prough, R.A., Cave, M.C. PCBs Alter EGFR Signaling in Organopollutant-Mediated Steatohepatitis. (Poster: Research! Louisville. 2015.)

Clair, H.B., Falkner, K.C., Prough, R.A., Pinkston, C., Brock, G.N., Pavuk, M., Dutton, N., Cave, M.C. Polychlorinated biphenyl exposure in Anniston, Alabama is associated with elevated prevalence of liver Disease. Hepatology. 2015;61(1):205A. (Poster: 65 $5^{\text {th }}$ Annual Meeting of the American Association For The Study Of Liver Disease. November 15-17. San Francisco, CA).

Hardesty, J., Falkner, K.C., Clair, H.B., Wahlang, B., Prough, R.A., Cave, M.C. Organopollutant Exposures Further Decrease Epidermal Growth Factor Signaling in Non-Alcoholic Steatohepatitis and Alter Hepatic Energy Metabolism. Hepatology. 2015;61(1):116A. (Poster: $65^{\text {th }}$ Annual Meeting of the American Association For The Study Of Liver Disease. November 1517. San Francisco, CA). 
Clair, HB; Falkner, KC; Prough, RA; Cave, M. Serum Steatohepatitis Biomarker and Proinflammatory Cytokine Elevation In Response To Polychlorinated Biphenyl Exposures in the Anniston Community Health Study (ACHS). The Toxicologist CD Supplement to Toxicological Sciences - An Official Journal of the Society of Toxicology. 2014; 138(1). Abstract no. 1569. (Poster: $53^{\text {rd }}$ Annual Meeting of the Society of Toxicology. Phoenix, AZ. March 23-27, 2014.)

Cave, M; Al-Eryani, L; Wahlang, B; Clair, H; Falkner, KC; Prough, RA. Identification of Pesticides and Other Environmental Chemicals Associated With The Development of Nonalcoholic Fatty Liver Disease in Rodents. The Toxicologist CD Supplement to Toxicological Sciences - An Official Journal of the Society of Toxicology. 2014; 138(1). Abstract no. 676. (Poster: $53^{\text {rd }}$ Annual Meeting of the Society of Toxicology. Phoenix, AZ. March 23-27, 2014.)

Song, M; Al-Eryani, L; Clair, H; Guardiola, J; Falkner, KC; Prough, RA; States, J; Cave, M. Aroclor 1260 Exposure Causes Steatohepatitis and Activates Hepatic Receptors in an Animal Model of Diet-Induced Obesity. The Toxicologist CD Supplement to Toxicological Sciences - An Official Journal of the Society of Toxicology. 2014; 138(1). Abstract no. 1870. (Poster: $53^{\text {rd }}$ Annual Meeting of the Society of Toxicology. Phoenix, AZ. March 23-27, 2014.)

Al-Eryani, L., Wahlang, B., Falkner, K.C., Clair, H., Prough, R.A., States, J.C., Cave, M. Identification of Environmental Chemicals Which Could Contribute to Nonalcoholic Fatty Liver Disease by Nuclear Receptor Activation. The Toxicologist CD Supplement to Toxicological Sciences An Official Journal of the Society of Toxicology. 2014; 138(1). Abstract no. 1159. (Poster: $53^{\text {rd }}$ Annual Meeting of the Society of Toxicology. Phoenix, AZ. March 23-27, 2014.)

Wahlang, B; Falkner, KC; Clair, HB; Al-Eryani, L; Guardiola, JJ; Prough, RA; Cave, MC. Hepatic Receptor Activation By Polychlorinated Biphenyls Implications For Xenobiotic/Energy Metabolism And Nonalcoholic Fatty Liver Disease. (Poster: $64^{\text {th }}$ Annual Meeting of the American Association For The Study Of Liver Disease. Washington, DC 2013.)

Al-Eryani, L; Wahlang, B; Clair, HB; Guardiola, JJ; Falkner, KC; Prough, RA; Cave, MC. Identification And Validation Of Environmental Chemical Nuclear Receptor Agonists Which Could Contribute To Nonalcoholic Fatty Liver Disease. (Poster: $64^{\text {th }}$ Annual Meeting of the American Association For The Study Of Liver Disease. Washington, DC 2013.)

Wahlang, B; Song, M; Beier, Jl; Al-Eryani, L; Clair, HB; Guardiola, JJ; Falkner, KC; Prough, RA; Cave, MC. Aroclor 1260 Exposure Worsens Hepatic And Systemic Inflammation In An Animal Model Of Diet-Induced Obesity And Nonalcoholic Fatty Liver Disease. (Poster: $64^{\text {th }}$ Annual Meeting of the American Association For The Study Of Liver Disease. Washington, DC 2013.) 
Al-Eryani, L.; Wahlang, B; Clair, HB; Guardiola, JJ; Falkner, KC; Prough, RA; Cave, MC. Identification Of Pesticides And Other Environmental Chemicals Associated With The Development Of Nonalcoholic Fatty Liver Disease In Rodents. (Poster: $64^{\text {th }}$ Annual Meeting of the American Association For The Study Of Liver Disease. Washington, DC 2013.)

Cave, MC; Beier, Jl; Wheeler, B; Falkner, KC; Bellis-Jones, HJ; Clair, HB; McClain, CJ; Occupational vinyl chloride exposures are associated with significant changes to the plasma metabolome: implications for toxicant associated steatohepatitis. (Poster: $63^{\text {rd }}$ Annual Meeting of the American Association For The Study Of Liver Disease. Boston, MA 2012.)

Falkner, KC; Wahlang, B; Bellis-Jones, HJ; Clair, HB; Prough, RA; Cave, MC. LXR and PXR crosstalk on DR4 response elements is sequence dependent. (Poster: $18^{\text {th }}$ North American Regional International Society For The Study Of Xenobiotics. Dallas, TX 2012.)

Clair, HB; Wahlang, B; Falkner, KC; Cave, M. An optimized DR 4 nuclear response element is bound and activated by PXR and LXR in the presence of known ligands, but not polychlorinated biphenyls. (Poster: Research Louisville. Louisville, KY 2012)

Goel, M; Hamid, T; Ismahil, MA; Zhou, GH; Brittian, K; Clair, H; Guo, SZ; Prabhu, SD. Selective macrophage ablation attenuates diet-induced obesity and insulin resistance and reverses diabetic cardiomyopathy. Circulation supplement, published Nov. 22, 2011.

Clair, HB; Herbert, D; Sag, D; Hansen, R; Suttles, J. C68-driven expression of a dominant-negative AMPKa1 protein results in spontaneous obesity and hepatosteatosis. Research Louisville. Louisville, KY 2011.

Zhou, GH; Keskey, AL; Goel, M; Hamid, T; Guo, SZ; Clair, HB; Brittian, KR; Sansbury, BE; Hill, BG; Prabhu, SD. Endoplasmic reticulum (ER) stress is critical for the development of diabetic cardiomyopathy. Circulation supplement, published Nov. 23, 2010.

Gozal LK, Bhattacharjee R, Kim J, Clair H, Gozal, D. Resident bone marrow stem cell are recruited to peripheral circulation in children with OSA : relevance to endothelial function. Sleep supplement, published 2010.

Nair, D.; Li, R.; Clair, H.; Cheng, Y; Gozal, D. Neuroglobin overexpression attenuates cognitive deficits in mice exposed to chronic intermittent hypoxia during sleep. Sleep supplement, published 2010.

Kingery, JR; Lewis, RK; Ismahil, MA; Guo, SZ; Clair, H; Hamid, T; Prabhu, SD. Inflammatory Cell-Localized Inducible Nitric Oxide Synthase

Exacerbates Post-Infarction Left Ventricular Remodeling. Abstract: 82nd Scientific Session of the American-Heart-Association. Orlando, FL 2009. 
Lewis RK, Kingery JR, Ismahil MA, Guo SZ, Clair H, Brittian K, Henning A, Hamid T, Prabhu SD. Inflammatory cell-localized Tumor Necrosis Factor Receptors 1 and 2 have divergent effects on post-infarction left ventricular remodeling. Circulation supplement published Nov. 3, 2009.

Gozal D, Gharib S, Dayyal E, Boazza M, Clair H, Kucia M, Khalyfa A. Gene networks and biological pathways in bone marrow-derived very small embryonic stem cells (VSEL) from mice following intermittent hypoxia (IH). (Poster: $23^{\text {rd }}$ Annual Meeting of the Associated Professional Sleep Societies. Seattle, WA 2009.) 